UNITED STATES DEPARTMENT OF THE INTERIOR

RAY LYMAN WILBUR, Secretary

GEOLOGICAL SURVEY

W. C. MENDENHALL, Director

Water-Supply Paper 695

\title{
SURFACE WATER SUPPLY of HAWAII
}

JULY 1, 1928, to JUNE 30, 1929

NATHAN C. GROVER, Chief Hydraulic Engineer MAX H. CARSON, District Engineer

Prepared in cooperation with the TERRITgF OW HAWAII

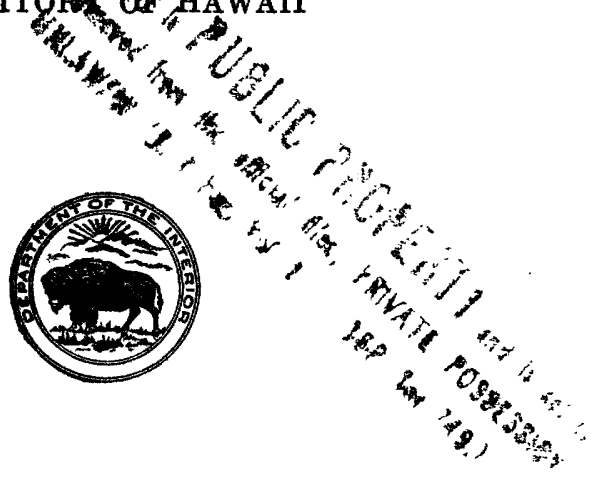

UNITEDD STATES

GOVERNMENT PRINTING OFFICE

WASHINGTON : 1932 


,




\section{CONTENTS}

Authority for investigations.

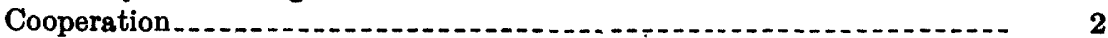

Cooperation with the Territory of Hawaii

Other cooperation... 3

Scope of work

Definition of terms...... 4

Explanation of data. ...

Accuracy of field data and computed results... 6

Division of work _.

Publications.... 7

Gaging-station records. ......

Island of Kauai .

Waimea River below Kekaha ditch intake, near Waimea...... 8

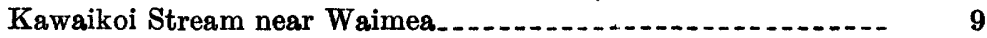

Kokee ditch near Waimea.................................. 10

Waiahulu Stream above Koaie Stream, near Waimea.......... 11

Koaie Stream at elevation 3,700 feet, near Waimea_........ 12

Waialae River at elevation 3,700 feet, near Waimea......... 13

Kekaha ditch at camp No. 1, near Waimea............... 14

Kekaha ditch below tunnel No. 12, near Waimea.......... 15

Hanapepe River at Koula, near Eleele.

Hanapepe ditch at Koula, near Eleele.......... 17

South Fork of Wailua River near Lihue._. 18

North Fork of Wailua River at elevation 650 feet, near Lihue_- $\quad 19$

Kanaha ditch near Lihue................. 20

East Branch of North Fork of Wailua River near Lihue._._..- 21

Kapahi ditch near Kealia............

Anahola River near Kealia..........

Anahola ditch above Kaneha Reservoir, near Kealia.......... 24

Hanalei River at elevation 625 feet, near Hanalei.......... 25

Waioli Stream near Hanalei........

Lumahai River near Hanalei...

Miscellaneous measurements..... 28

Island of Oahu..... 30

Right Branch of North Fork of Kaukonahua Stream near

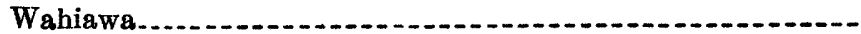

Left Branch of North Fork of Kaukonahua Stream near

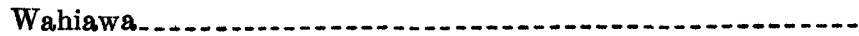

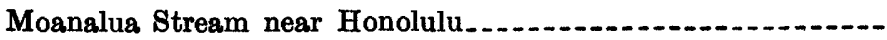

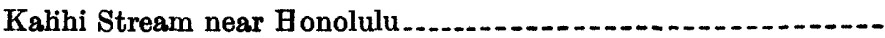

Nuuanu Stream below Reservoir No. 2 wasteway, near Honolulu..

West Branch of Manoa Stream near Honolulu.........

East Branch of Manoa Stream near Honolulu.........

East Manoa ditch near Honolulu.........

Pukele Stream near Honolulu. 
Gaging-station records-Continued.

Island of Oahu-Continued.

Waiomao Stream above Pukele Stream, near Honolulu .....--

Miscellaneous measurements. .

Island of Molokai.

Halawa Stream near Halawa.

Papalaua Stream near Wailau

Waiakeakua Stream near Wailau

Pulena Stream near Wailau.....

Pelekunu Stream near Pelekunu.:-

Lanipuni Stream near Pelekunu.....

Waikolu Stream at pipe-line crossing near Kalaupapa..........

Miscellaneous measurements...

Island of Maui

Honokahau Stream near Honokahau.........

Honokawai ditch near Lahaina. . . . . . . . .

Kanaha Stream above pipe-line intake near Lahaina.........-.

Olowalu ditch near Olowalu. . . .

Oheo Stream at elevation 1,550 feet, near Kipahulu...........

Right Branch of Kahalawe Stream near Kipahulu............

Hanawi Stream near Nahiku.........

Kapaula Stream near Nahiku

Koolau ditch at Nahiku weir, near Nahiku................

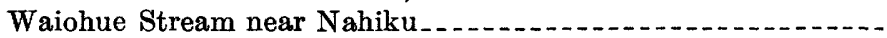

West Kopiliula Stream near Keanae.

East Wailuaiki Stream near Keanae..

West Wailuaiki Stream near Keanae..........

East Wailuanui Stream near Keanae......

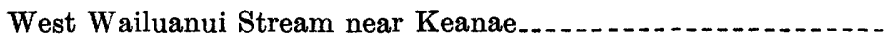

Koolau ditch near Keanae........

Honomanu Stream near Keanae........

Haipuaena Stream near Huelo

Spreckels ditch at Haipuaena weir, near Huelo............

Puohokamoa Stream near Huelo.

Puohokamoa intake of Koolau ditch near Huelo............

Manuel Luis ditch at Puohokamoa Gulch, near Huelo.........

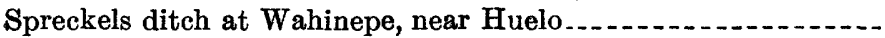

Koolau ditch at Wahinepe, near Huelo...

Alo Stream near Huelo. .

Waikamoi Stream above Wailoa ditch, near Huelo..........

Kaaiea Stream near Kailua. . . . .

Spreckels ditch below Kaaiea Gulch, near Huelo..............

Center ditch below Kolea Reservoir, near Huelo............

Nailiilihaele Stream near Huelo... .

Kailua Stream near Huelo._.

Hoolawaliilii Stream near Huelo.........

Hoolawanui Stream near Huelo......

Honopou Stream near Huelo.........

Wailoa ditch at Honopou, near Huelo........

New Hamakua ditch at Honopou, near Huelo..............

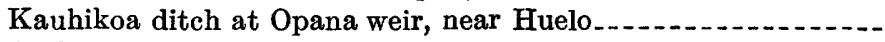

Haiku ditch at Manawai Gulch, near Peahi

Miscellaneous measurements. 
Gaging-station records-Continued.

Island of Hawaii ...

Wailuku River above Hilo Boarding School ditch intake, near Hilo

Kapehu Stream at Piihonua, near Hilo

Honolii Stream near Hilo

Awini ditch at East Honokaneiki Gulch, near Niulii

East Honokaneiki intake to Awini ditch at East Honokaneiki

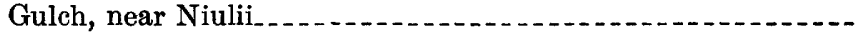

Kohala ditch at Pololu, near Niulii................. 95

Kehena ditch near Kohala_._._._.

Index.

Miscellaneous measurement 


\section{SURFACE WATER SUPPLY OF HAWAII, JULY 1, 1928, T0 JUNE 30, 1929}

\section{AUTHORITY FOR INVESTIGATIONS}

This volume contains records of measurements of flow made on certain streams and ditches in the Territory of Hawaii during the year ending June 30, 1929. The data presented in this report were collected by the United States Geological Survey in cooperation with the Territory of Hawaii, under the general sanction of the organic law of the Geological Survey (20 Stat. L., p. 394), which contains the following paragraph:

Provided, That this officer [the Director] shall have the direction of the Geological Survey and the classification of public lands and examination of the geological structure, mineral resources, and products of the national domain.

As water is the most abundant and most valuable of the minerals, the investigation of water resources is authorized under the provision for examining mineral resources. Since the fiscal year ending June 30,1895 , successive appropriation bills passed by Congress have carried the following item:

For gaging the streams and determining the water supply of the United States, and for the investigation of underground currents and artesian wells, and for the preparation of reports upon the best methods of utilizing the water resources.

For the fiscal years ending June 30, 1929, and thereafter the appropriation bills have carried, in addition to the above provisions, the following proviso: "Provided, That no part of this appropriation shall be expended in cooperation with States or municipalities except upon the basis of the State or municipality bearing all the expense incident thereto in excess of such an amount as is necessary for the Geological Survey to perform its share of general water resources investigations, such share of the Geological Survey in no case exceeding 50 per centum."

The Legislature of the Territory of Hawaii approved on March 22, 1909 , "An act to promote the conservation and development of the natural resources of the Territory," which provided in substance as follows: A special tax of 2 per cent shall be levied, assessed, and collected annually on all incomes in excess of $\$ 4,000$; and all amounts so collected shall constitute a special fund to be expended only for the encouragement of immigration and the conservation of natural resources in the proportion of three-fourths for immigration and one- 
fourth for conservation. The conservation fund shall be used for the development, conservation, improvement, and utilization of the natural resources, and shall be available for expenditure at such times and in such manner as a board of three persons appointed in accordance with section 80 of the organic act shall, with the approval of the governor, determine.

An act of April 26, 1911, amended the original act so as to extend it until December 31, 1913.

On April 4, 1913, the Governor of the Territory of Hawaii approved Act 56 , for the creation and maintenance of a division of hydrography under the board of agriculture and forestry, and Act 57, appropriating the revenues from water licenses for the use of the board of commissioners of agriculture and forestry toward forest protection and hydrographic surveying.

Since June 30,1915 , the funds for the use of the division of hydrography have been supplied by successive appropriations from the general revenues of the Territory.

On March 23, 1917, the following act (Act 27) by the Legislature of the Territory of Hawaii was approved:

Secrion 1. The division of hydrography, authorized by and created pursuant to section 483 of the Revised Laws of Hawaii, 1915, is hereby transferred, together with all the materials, equipment, and supplies now under the control of the division or of the board of commissioners of agriculture and forestry for the division, to the commissioner of public lands.

Sec. 2. The commissioner of public lands shall have and exercise the same powers, duties, and jurisdiction with respect to said division as are now exercised by the board of commissioners of agriculture and forestry.

SEc. 3. All unexpended balances of appropriations heretofore made for said division, the expenditure of which is now by law vested in the board of commissioners of agriculture and forestry, are hereby transferred to the commissioner of public lands and the expenditure thereof vested in said commissioner.

SEc. 4. This act shall take effect upon its approval.

\section{COOPERATION}

\section{COOPERATION WITH THE TERRITORY OF HAWAII}

Under the authority conferred by the Federal and Territorial legislation, the Director of the United States Geological Survey and the Governor of the Territory of Hawaii entered into a cooperative agreement, dating from July 1, 1910, for "the gaging of streams and the determination of the water supply of the Territory of Hawaii."1

The principal features of this agreement are:

1. The United States Geological Survey assumes the responsibility of gathering, analyzing, and publishing the data.

1 The United States Geological Survey also cooperated with the Territory of Hawaii in mapping the eight main islands. 
2. During the progress of the work all notes, maps, and data gathered as a result of field studies are at all times open to inspection by the representative of the Territory, and if they are not satisfactory the agreement can be terminated.

3. Accounts for payment of salaries, travel, and subsistence, supplies, or other expenses necessary to the completion of the work shall be rendered in the manner required by the laws and regulations of the contracting parties, and vouchers shall be proffered to either party for payment according as it may be convenient or according to the balance remaining in the respective allotments.

4. The cost of publication is borne entirely by the Geological Survey.

Unless otherwise stated, all data in this paper have been collected and are published under this cooperative agreement with the Territory of Hawaii.

Until June 30,1913, the Territory of Hawaii was represented in the cooperation by the board of conservation; from July 1, 1913, to March 23, 1917, by the board of commissioners of agriculture and forestry; and since this date by the commissioner of public lands.

\section{OTHER COOPERATION}

Some of the data in this paper have been obtained in cooperation with the city and county of Honolulu, the city of Hilo, and private persons and corporations, under one of the plans indicated in the following paragraphs:

1. Expense of work, equipment, and installation paid entirely or in part by the cooperating party.

2. Records collected by employees of a cooperating party but under supervision of and by methods of the Survey.

3. Assistance given in the collection of records, such as furnishing transportation, subsistence, and equipment.

4. Records furnished by a cooperating party, collected by his methods and under his supervision.

Cooperation in the collection of records for whose accuracy responsibility has not rested with the Geological Survey has been acknowledged in the descriptions of the stations. Special acknowledgment is due to the following organizations cooperating under plans 1, 2, and 3: Island of Kauai-Kekaha Sugar Co., McBryde Sugar Co., East Kauai Water Co., Princeville Plantation Co., and American Factors (Ltd.); Island of Oahu-city and county of Honolulu, Board of Water Supply, B. P. Bishop Estate, and Wahiawa Water Co. ; Island of MauiPioneer Mill Co. and East Maui Irrigation Co.; Island of HawaiiHilo Waterworks, C. Brewer \& Co. (Ltd.), and Kohala Ditch Co. 


\section{SCOPE OF WORK}

Since the beginning of stream-gaging work in Hawaii in 1910, records of flow of streams and ditches have been obtained at about 400 stations for periods ranging from a few months to 19 years. In addition hundreds of miscellaneous measurements have been made, and rather extensive studies of ground water have been made in Kau, Hawaii, and in Honolulu, Oahu.

In this volume are given the records of daily flow that were obtained at the 83 stations that were operated during the year ending June 30,1929 , and the results of miscellaneous measurements of stream flow made during that year. The results of ground-water studies will be published in separate water-supply papers. (See p. - for a record of other water-supply papers pertaining to Hawaii.)

\section{DEFINITION OF TERMS}

The volume of water flowing in a stream-the "run-off" or "discharge"-is expressed in various terms, each of which has become associated more or less definitely with a certain class of work. These terms may be divided into two groups: (1) Those that represent a rate of flow, as "second-feet," "gallons a minute," "gallons a day," "miner's inches," and "run-off in second-feet a square mile," and (2) those that represent the actual quantity of water, as "run-off in inches," "million gallons," and "acre-feet." Those used in this report may be defined as follows:

"Second-foot" is an abbreviation for cubic foot a second and is the unit for the rate of discharge of water flowing in a stream 1 square foot in cross section at a rate of 1 foot a second. It is generally adopted as the fundamental unit in the measurement of flowing water and is the "natural" unit, as the foot and the second are the units used in making the physical determinations.

An "acre-foot" is equivalent to 43,560 cubic feet and is the quantity required to cover an acre to the depth of 1 foot. The term is commonly used in connection with storage for irrigation.

In the Territory of Hawaii the unit most commonly used in measuring water is the "million gallons." This is used with two meanings-(1) to indicate a rate of flow and (2) to express an actual quantity of water. In the former sense "million gallons a day" is inferred, 1,000,000 gallons being taken as the unit of quantity and 24 hours as the unit of time. With this meaning the term is generally used in connection with pumping and irrigation. In the latter sense "million gallons" as an absolute quantity is used in the measurement of storage capacities of reservoirs.

The following convenient approximate relations exist between second-feet, million gallons a day, and acre-feet; 1 second-foot 
flowing 24 hours equals about 2 acre-feet; $1,000,000$ gallons equals about 3 acre-feet; and 1 second-foot equals approximately two-thirds of $1,000,000$ gallons a day.

The following terms not in common use are here defined:

"Stage-discharge relation," an abbreviation for the term "relation of gage height to discharge."

"Control," a term used to designate the section or sections of the stream channel below the gage which determine the stage-discharge relation at the gage. It should be noted that the control may not be the same section or sections at all stages.

The "point of zero flow" for a gaging station is that point on the gage-the gage height-at which water ceases to flow over the control.

\section{EXPLANATION OF DATA}

The base data collected at gaging stations consist of records of stage, measurements of discharge, and general information used to supplement the gage heights and discharge measurements in determining the daily discharge. The records of stage used in computing discharges in this paper are obtained from water-stage recorders that give continuous records of the fluctuations. Measurements of discharge are made with a current meter by the general methods outlined in standard textbooks on the measurement of river discharge. Occasionally discharge is determined from a weir, using weir formulas.

From the discharge measurements, rating tables are prepared that give the discharge for any stage. The application of the daily gage heights to these rating tables gives the discharge from which the daily, monthly, and yearly discharges are determined.

The data presented in this report comprise, for each gaging station, a description of the station, a table showing the daily discharge of the stream, and a table of monthly and yearly discharge and run-off. All rates of flow are expressed as million gallons a day.

The description of the station gives location, drainage area, records available, discharge corresponding to maximum and minimum recorded stages, and, under "Remarks," notes on accuracy of the records, diversions that decrease the flow at the gage, and artificia regulation.

The table of daily discharge gives, in general, the discharge corresponding to the mean daily gage heights. At stations on streams subject to sudden or rapid diurnal fluctuation the discharge obtained from the rating table by applying the mean daily gage height may not be the true mean discharge for the day. At such stations the mean daily discharge may be obtained by averaging discharge for intervals during the day or by use of the discharge integrator, an instrument operating on the principle of the planimeter and containing as an essential element the rating curve of the station. 
In the table of monthly discharge the column headed "Maximum" gives the flow for the day when the total discharge was greatest. This does not correspond to the rate of flow at the crest of the flood. The maximum rate of flow is given in the station description under the heading "Extremes," and the corresponding stage is always taken from the water-stage recorder graph unless otherwise noted. Likewise, in the column headed "Minimum" the quantity given is the flow for the day when the total discharge was least. The columns headed "Mean" give the average flow in million gallons a day and cubic feet a second during the month. The "total in million gallons" is the sum of the daily flows, and the "total in acre-feet" is computed from the mean monthly discharge in million gallons a day.

\section{ACCURACY OF FIELD DATA AND COMPUTED RESULTS}

The accuracy of stream-flow data depends primarily (1) on the permanence of the stage-discharge relation, and (2) on the accuracy of observation of stage, measurements of flow, and interpretation of records.

Permanence of the stage-discharge relation will be affected by any change in the control due to growth of vegetation in the stream bed, effects of floods, or any artificial changes, and it may be affected by changes in gage datum.

Observations of stage are taken from the water-stage recorder graphs, with a scale of gage heights so chosen as to give less than 2 per cent error. However, this accuracy may be interfered with by unsatisfactory operation of water-stage recorders or by plugged or sluggish intakes to stilling wells.

In general, measurements of flow by current meter give less than 5 per cent error except where it is impossible to find suitable measuring conditions. Rating curves are usually well defined, except for extremely low or high stages, by current-meter measurements and are extended by the use of area and velocity curves, slope measurements, weir tables, logarithmic curves, comparison with previous curves, knowledge of the station, or any combination of these methods.

Unless otherwise noted daily discharges are ascertained by applying to the rating table mean daily gage heights obtained from the recorder graph by inspection or, for days of considerable fluctuation in stage, by averaging discharges for intervals of the day. All computations are carried to three significant figures except where this would require the use of more than two decimal places. The discharges thus obtained are plotted, usually on semi-logarithmic paper, for comparison with the flow of comparable streams, and any inconsistencies that appear are verified or corrected.

A general statement under "Remarks" gives the accuracy of records, based on the above information, the terms "excellent," "good," "fair," 
or "poor," indicating that the record is probably accurate within 5 , 10,15 , and 20 per cent, respectively.

It should be borne in mind that the observations in each succeeding year may be expected to throw new light on data previously published.

\section{DIVISION OF WORK}

The data were collected and prepared for publication under the direction of M. H. Carson, district engineer, Honolulu, Hawaii, by W. E. Armstrong, office engineer, K. N. Valsvik, J. H. Hofmann, K. M. Kelley, Sam Wong, H. W. Palm, G. T. Hirashima, Kenichi Kawamura, John Kaheaku, P. T. Goo, and Miss M. A. Davidson.' The manuscript has been prepared by W. E. Armstrong and reviewed by M. H. Carson.

\section{PUBLICATIONS}

The following table gives by years the numbers of the papers on the surface-water supply of Hawaii containing data from 1903 to 1929 , and used in conjunction with the list of stations maintained (see Water-Supply Paper 595) provides a convenient index for finding the data for any station. The data for any particular station will be found in the reports covering the years during which the station was maintained except when publication is delayed owing to undeveloped rating curves. Occasionally data are revised and republished in later papers. Miscellaneous discharge measurements made during any year at points other than regular gaging stations are published in the paper containing that year's data.

Numbers of water-supply papers containing data on the surface-water supply of Hawaii, 1903-1929

\begin{tabular}{|c|c|c|c|c|c|}
\hline Year & Number & Year & Number & Year & Number \\
\hline $\begin{array}{l}1903 \\
1909-1911 \text { b } \\
1912 b \\
19136 \\
1913-1915 \\
1915-16 \\
1916-17-15\end{array}$ & $\begin{array}{l}\text { a } 77 \\
318 \\
336 \\
373 \\
430 \\
445 \\
465\end{array}$ & $\begin{array}{l}1917-18 \\
1918-19 \\
1919-20 \\
1920-21 \\
1921-22 \\
1922-23 \\
1923-24\end{array}$ & $\begin{array}{l}485 \\
515 \\
516 \\
535 \\
555 \\
575 \\
595\end{array}$ & \begin{tabular}{|l}
$1924-25$ \\
$1925-26$ \\
$1926-27$ \\
$1927-28$ \\
$1928-29$
\end{tabular} & $\begin{array}{l}615 \\
635 \\
655 \\
675 \\
695\end{array}$ \\
\hline
\end{tabular}

a Water resources of Molokai, by Waldemar Lindgren.

- Calendar years; reports subsequent to Water-Supply Paper 373 cover the year beginning July 1 and ending June $\mathbf{3 0}$. 


\section{GAGING-STATION RECORDS}

\section{ISLAND OF KAUAI}

WAIMEA RIVER BELOW KEKAHA DITCH INTAKE, NEAR WAIMEA, KAUAI

Location.-Water-stage recorder in Waimea Canyon, 500 feet below Kekaha ditch intake and 8 miles by trail north of Waimea.

Drain Age AREA. - 45.0 square miles.

ReCORDS AVAILABLe.-July, 1921, to June, 1929.

ExTREmes.-Maximum discharge during year, 1,940 million gallons a day or 3,000 second-feet Nov. 4 (gage height, 16.20 feet); minimum, 0.2 million gallons a day or 0.3 second-foot May 20-22.

1921-1929: Maximum discharge, 2,770 million gallons a day or 4,290 second-feet Dec. 24, 1927 (gage height, 20.40 feet); no flow several days from July to November, 1926.

Remarks.-Records good for medium stages except those estimated; all estimated and extremely high and low-stage records poor. Kokee ditch and Kekaha ditch divert above the station, taking practically all the water at low and medium stages for irrigation near Waimea.

Discharge, in million gallons a day, 1928-29

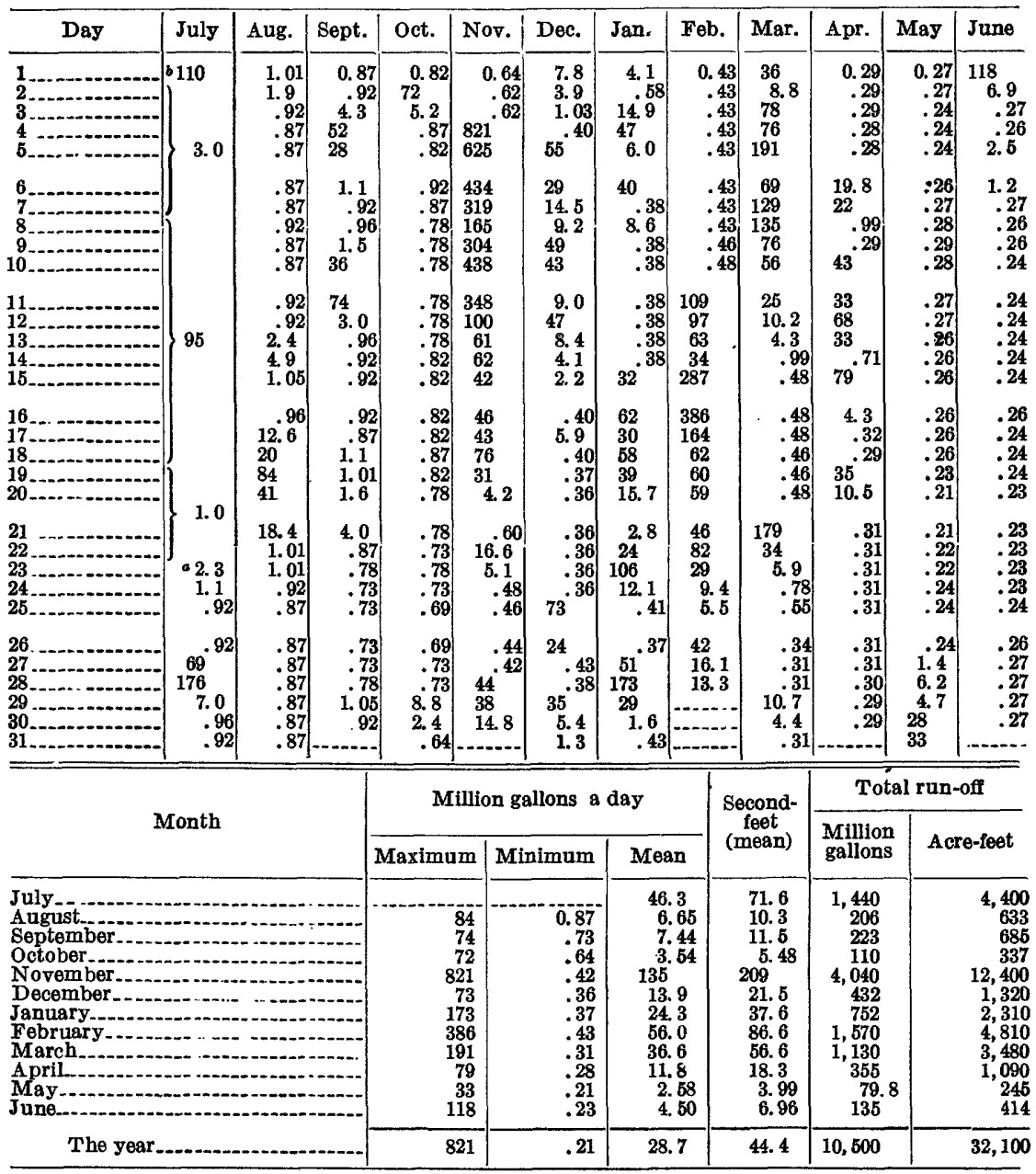


KAWATKOI STREAM NBAR WAIMA, KAUAI

LOCATION.-Water-stage recorder 2 miles northeast of Kokee ranger station and 12.5 miles northeast of Waimea.

Drainage AREA.- 4.1 square miles.

RirCords available.-April, 1909, to June, 1929. July, 1917, to July, 1919, not published.

Extremes.-Maximum discharge during year, 1,590 million gallons a day or 2,460 second-feet Nov. 4 (gage height, 11.11 feet); minimum, 1.6 million gallons a day or 2.5 second-feet Oct. 16 .

1909-1929: Maximum discharge, 1,670 million gallons a day or 2,580 second-feet Dec. 13, 1924 (gage height, 12.11 feet); minimum, 1.3 million gallons a day or 2.0 second-feet Sept. 15, 1921.

Highest known flood, 15.2 feet Dec. 18, 1916 (discharge not determined). REMARKs.-Records good for ordinary stages. No diversions above station.

Discharge, in million gallons a day, 1928-29

\begin{tabular}{|c|c|c|c|c|c|c|c|c|c|c|c|c|}
\hline Day & July & Aug. & Sept. & Oct. & Nov. & Dec. & Jan. & Feb. & Mar. & Apr. & May & June \\
\hline $\begin{array}{l}2 \\
3 \\
4 \\
5\end{array}$ & $\begin{array}{r}12.9 \\
10.3 \\
5.2 \\
4.5 \\
6.9\end{array}$ & $\begin{array}{r}41 \\
18.3 \\
9.6 \\
7.8 \\
6.9\end{array}$ & $\begin{array}{l}4.5 \\
22 \\
13.6 \\
58 \\
16.2\end{array}$ & \begin{tabular}{r|}
4.4 \\
11.0 \\
5.5 \\
3.3 \\
3.0
\end{tabular} & $\begin{array}{r}5.1 \\
2.9 \\
2.1 \\
634 \\
390\end{array}$ & $\begin{array}{r}7.8 \\
6.9 \\
6.3 \\
14.1 \\
62\end{array}$ & $\begin{array}{l}5.9 \\
5.6 \\
72 \\
37 \\
11.0\end{array}$ & $\begin{array}{l}6.7 \\
6.3 \\
5.9 \\
5.6 \\
5.2\end{array}$ & $\begin{array}{l}11.9 \\
14.4 \\
83 \\
33 \\
143\end{array}$ & $\begin{array}{l}5.4 \\
4.9 \\
4.5 \\
4.3 \\
4.1\end{array}$ & $\begin{array}{l}\text { 3. } 7 \\
3.6 \\
3.5 \\
3.6 \\
4.0\end{array}$ & $\begin{array}{l}33 \\
8.8 \\
5.8 \\
6.3 \\
30\end{array}$ \\
\hline $\begin{array}{r}6 \\
7 \\
8 \\
8 \\
9\end{array}$ & $\begin{array}{c}5.5 \\
55 \\
148 \\
17.2 \\
27\end{array}$ & $\begin{array}{l}6.2 \\
6.3 \\
5.5 \\
4.5 \\
4.2\end{array}$ & $\begin{array}{r}7.9 \\
19.2 \\
15.8 \\
6.2 \\
4.7\end{array}$ & $\begin{array}{l}3.4 \\
2.7 \\
2.3 \\
2.1 \\
2.1\end{array}$ & $\begin{array}{l}215 \\
172 \\
107 \\
204 \\
315\end{array}$ & $\begin{array}{l}38 \\
17.1 \\
37 \\
63 \\
26\end{array}$ & $\begin{array}{r}7.9 \\
8.2 \\
41 \\
10.3 \\
7.6\end{array}$ & $\begin{array}{l}5.0 \\
4.6 \\
4.5 \\
4.2 \\
6.1\end{array}$ & $\begin{array}{l}53 \\
84 \\
65 \\
51 \\
32\end{array}$ & $\begin{array}{r}3.9 \\
3.7 \\
3.6 \\
9.3 \\
90\end{array}$ & $\begin{array}{r}3.7 \\
7.8 \\
24 \\
6.9 \\
7.0\end{array}$ & $\begin{array}{r}13.4 \\
7.6 \\
4.9 \\
4.0 \\
4.2\end{array}$ \\
\hline L_. & $\begin{array}{l}72 \\
19.3 \\
16.3 \\
47 \\
23\end{array}$ & $\begin{array}{r}7.4 \\
6.0 \\
7.8 \\
12.4 \\
12.7\end{array}$ & $\begin{array}{r}22 \\
7.1 \\
4.4 \\
3.8 \\
4.2\end{array}$ & $\begin{array}{l}2.0 \\
1.9 \\
1.8 \\
1.6 \\
1.6\end{array}$ & $\begin{array}{r}168 \\
45 \\
56 \\
42 \\
30\end{array}$ & $\begin{array}{l}15.5 \\
25 \\
11.9 \\
17.6 \\
13.6\end{array}$ & $\begin{array}{r}7.2 \\
6.4 \\
5.7 \\
6.3 \\
56\end{array}$ & \begin{tabular}{|c|}
100 \\
25 \\
12.0 \\
8.4 \\
48
\end{tabular} & $\begin{array}{l}18.7 \\
22 \\
22 \\
12.4 \\
10.3\end{array}$ & $\begin{array}{l}32 \\
88 \\
23 \\
45 \\
58\end{array}$ & $\begin{array}{r}6.7 \\
4.5 \\
3.6 \\
8.2 \\
13.3\end{array}$ & $\begin{array}{l}23 \\
23 \\
13.0 \\
7.3 \\
6.0\end{array}$ \\
\hline 16 & $\begin{array}{c}94 \\
137 \\
28 \\
14.3 \\
10.5\end{array}$ & $\begin{array}{r}7.3 \\
5.1 \\
15.9 \\
20 \\
6.2\end{array}$ & $\begin{array}{l}4.2 \\
4.1 \\
3.7 \\
3.0 \\
2.8\end{array}$ & $\begin{array}{l}1.6 \\
1.8 \\
3.4 \\
2.5 \\
2.0\end{array}$ & $\begin{array}{l}26 \\
62 \\
63 \\
24 \\
16.5\end{array}$ & $\begin{array}{r}10.3 \\
10.6 \\
7.9 \\
6.9 \\
6.3\end{array}$ & $\begin{array}{l}62 \\
22 \\
27 \\
14.6 \\
9.4\end{array}$ & $\begin{array}{r}138 \\
68 \\
22 \\
56 \\
37\end{array}$ & $\begin{array}{l}9.4 \\
8.5 \\
7.8 \\
7.0 \\
7.3\end{array}$ & $\begin{array}{l}16.5 \\
10.1 \\
8.5 \\
22 \\
13.2\end{array}$ & $\begin{array}{l}6.0 \\
3.8 \\
3.1 \\
2.8 \\
2.5\end{array}$ & $\begin{array}{l}6.3 \\
5.6 \\
6.2 \\
5.2 \\
6.4\end{array}$ \\
\hline $\begin{array}{l}21 \\
22 \\
23 \\
24 \\
26\end{array}$ & \begin{tabular}{r|}
8.8 \\
7.6 \\
31 \\
10.3 \\
12.1
\end{tabular} & $\begin{array}{r}10.6 \\
9.4 \\
4.8 \\
3.8 \\
3.7\end{array}$ & $\begin{array}{l}3.4 \\
2.9 \\
2.6 \\
2.3 \\
2.1\end{array}$ & $\begin{array}{l}1.9 \\
2.7 \\
4.5 \\
2.7 \\
2.0\end{array}$ & $\begin{array}{l}29 \\
51 \\
17.4 \\
11.9 \\
9.9\end{array}$ & $\begin{array}{r}5.7 \\
5.4 \\
5.0 \\
5.0 \\
123\end{array}$ & $\begin{array}{l}32 \\
34 \\
36 \\
36 \\
12.9 \\
9.4\end{array}$ & $\begin{array}{l}24 \\
65 \\
21 \\
12.9 \\
10.5\end{array}$ & $\begin{array}{l}53 \\
12.9 \\
8.0 \\
6.9 \\
6.6\end{array}$ & $\begin{array}{l}7.8 \\
6.6 \\
5.9 \\
5.4 \\
5.0\end{array}$ & $\begin{array}{l}2.4 \\
2.4 \\
4.0 \\
3.1 \\
2.5\end{array}$ & $\begin{array}{l}3.8 \\
3.1 \\
2.7 \\
2.6 \\
5.0\end{array}$ \\
\hline $\begin{array}{l}26 \\
282 \\
28 \\
29 \\
30 \\
31\end{array}$ & $\begin{array}{l}17.3 \\
134 \\
50 \\
12.9 \\
8.8 \\
36\end{array}$ & $\begin{array}{l}4.5 \\
4.0 \\
3.4 \\
3.3 \\
3.5 \\
3.0\end{array}$ & $\begin{array}{l}2.1 \\
2.0 \\
2.3 \\
7.9 \\
8.4\end{array}$ & $\begin{array}{l}1.8 \\
1.6 \\
1.7 \\
6.6 \\
5.3 \\
3.5 \\
-\end{array}$ & $\begin{array}{r}9.0 \\
82.0 \\
32.2 \\
9.2\end{array}$ & $\begin{array}{r}20 \\
9.4 \\
8.0 \\
17.0 \\
9.2 \\
6.7\end{array}$ & $\begin{array}{r}8.2 \\
11.4 \\
55 \\
11.4 \\
8.5 \\
7.4\end{array}$ & \begin{tabular}{|c|}
24 \\
13.2 \\
19.1 \\
\hdashline... \\
\hdashline \\
\hdashline
\end{tabular} & $\begin{array}{r}6.3 \\
5.6 \\
5.9 \\
15.5 \\
9.6 \\
6.0\end{array}$ & $\begin{array}{l}4.6 \\
4.4 \\
4.1 \\
4.0 \\
3.9 \\
\cdots\end{array}$ & $\begin{array}{l}2.3 \\
6.0 \\
8.0 \\
32 \\
26 \\
44\end{array}$ & $\begin{array}{r}8.2 \\
6.5 \\
3.2 \\
2.8 \\
2.5 \\
- \\
-. .-\end{array}$ \\
\hline \multirow{2}{*}{\multicolumn{4}{|c|}{ Month }} & \multicolumn{4}{|c|}{ Million gallons a day } & \multirow{2}{*}{\multicolumn{2}{|c|}{$\begin{array}{c}\text { Second- } \\
\text { feet } \\
\text { (mean) }\end{array}$}} & \multicolumn{3}{|c|}{ Total run-off } \\
\hline & & & & Laximum & $\begin{array}{l}\mathrm{a} \\
\mathbf{n}\end{array}$ & nimum & Mean & & & $\begin{array}{l}\text { Million } \\
\text { gallons }\end{array}$ & Acre & e-feet \\
\hline \multicolumn{4}{|c|}{ 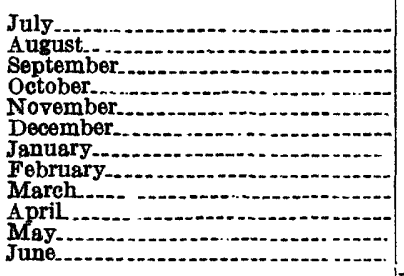 } & $\begin{array}{c}148 \\
41 \\
58 \\
11.0 \\
634 \\
123 \\
72 \\
138 \\
143 \\
90 \\
44 \\
33\end{array}$ & & $\begin{array}{l}4.5 \\
3.0 \\
2.0 \\
1.6 \\
2.1 \\
5.0 \\
5.6 \\
4.2 \\
5.6 \\
3.6 \\
2.3 \\
2.5\end{array}$ & $\begin{array}{r}34.9 \\
8.5 \\
8.7 \\
3.0 \\
92.3 \\
19.9 \\
20.9 \\
27.1 \\
26.8 \\
16.7 \\
8.2 \\
8.6\end{array}$ & & $\begin{array}{l}54.0 \\
13.2 \\
13.6 \\
4.70 \\
43 \\
30.8 \\
32.3 \\
41.9 \\
41.5 \\
25.8 \\
12.7 \\
13.4\end{array}$ & $\begin{array}{c}1,080 \\
265 \\
263 \\
94.3 \\
2770 \\
618 \\
649 \\
758 \\
832 \\
502 \\
255 \\
259\end{array}$ & & $\begin{array}{r}3,320 \\
813 \\
808 \\
289 \\
8.500 \\
1,890 \\
1,990 \\
2,330 \\
2,550 \\
\mathfrak{1}, 540 \\
783 \\
795\end{array}$ \\
\hline \multicolumn{4}{|c|}{ The year } & 634 & & 1.6 & 22.9 & & 35.4 & 8. 350 & & 25,600 \\
\hline
\end{tabular}




\section{KOKEE DITCH NBAR WAIMEA, KAUAT}

Location.-Water-stage recorder 1,000 feet west of road and 101/2 miles north of Waimea.

Records available.- September, 1926, to June, 1929.

Extremes.-Maximum discharge during year, 65 million gallons a day or 101 second-feet Nov. 4 and 5 (gage height, 2.62 feet); no flow for several short periods when water was shut out of ditch.

1926-1929: Maximum discharge, 68 million gallons a day or 105 secondfeet Dec. 24, 1927 (gage height, 2.70 feet); no flow occasionally when water was turned out of ditch just above weir.

REMarks.- Records excellent. Kokee ditch, at elevation 3,400 feet, diverts water from all streams tributary to Waimea River, east of and including Mohihi Stream, for irrigation near Kekaha. Regulated by head gates.

Discharge, in million gallons a day, 1928-29

\begin{tabular}{|c|c|c|c|c|c|c|c|c|c|c|c|c|}
\hline Day & July & Aug. & Sept. & Oct. & Nov. & Dec. & Jan. & Feb. & Mar. & Apr. & May & June \\
\hline $\begin{array}{l}1 \\
2 \\
2 \\
3 \\
4 \\
4 \\
5\end{array}$ & $\begin{array}{r}14.0 \\
15.6 \\
9.2 \\
8.1 \\
9.9\end{array}$ & $\begin{array}{l}45 \\
27 \\
15.1 \\
12.4 \\
11.4\end{array}$ & $\begin{array}{l}6.6 \\
22 \\
16.1 \\
29 \\
26\end{array}$ & $\begin{array}{r}7.6 \\
11.8 \\
10.0 \\
6.0 \\
5.1\end{array}$ & $\begin{array}{c}7.9 \\
5.6 \\
4.4 \\
40 \\
49\end{array}$ & $\begin{array}{l}15.9 \\
14.4 \\
13.3 \\
12.6 \\
44\end{array}$ & $\begin{array}{l}11.0 \\
10.5 \\
45 \\
43 \\
18.7\end{array}$ & $\begin{array}{l}14.9 \\
13.7 \\
13.1 \\
12.4 \\
11.8\end{array}$ & $\begin{array}{l}21 \\
19.8 \\
45 \\
42 \\
57\end{array}$ & $\begin{array}{r}12.0 \\
11.4 \\
10.5 \\
10.3 \\
9.9\end{array}$ & $\begin{array}{l}8.3 \\
8.1 \\
8.1 \\
8.1 \\
9.0\end{array}$ & $\begin{array}{r}39 \\
14.4 \\
9.3 \\
8.4 \\
28\end{array}$ \\
\hline $\begin{array}{r}6 \\
7 \\
8 \\
9 \\
10\end{array}$ & $\begin{array}{c}9.2 \\
36 \\
56 \\
28 \\
31\end{array}$ & $\begin{array}{r}10.5 \\
10.8 \\
10.6 \\
9.0 \\
8.4\end{array}$ & $\begin{array}{c}10.8 \\
18.1 \\
21 \\
9.8 \\
7.6\end{array}$ & $\begin{array}{l}5.6 \\
5.0 \\
4.4 \\
4.2 \\
4.4\end{array}$ & $\begin{array}{l}60 \\
60 \\
60 \\
60 \\
60\end{array}$ & $\begin{array}{l}42 \\
28 \\
40 \\
44 \\
38\end{array}$ & $\begin{array}{l}13.5 \\
12.0 \\
42 \\
18.7 \\
13.7\end{array}$ & $\begin{array}{l}11.4 \\
11.0 \\
10.8 \\
10.5 \\
12.3\end{array}$ & $\begin{array}{l}56 \\
56 \\
60 \\
56 \\
43\end{array}$ & $\begin{array}{c}9.7 \\
9.3 \\
9.3 \\
9.2 \\
50\end{array}$ & $\begin{array}{l}8.4 \\
10.5 \\
23 \\
11.6 \\
10.5\end{array}$ & $\begin{array}{r}18.7 \\
11.0 \\
8.3 \\
7.1 \\
6.9\end{array}$ \\
\hline $\begin{array}{l}11 \ldots \\
12 . . . \\
13 \\
14 \\
15\end{array}$ & $\begin{array}{l}46 \\
27 \\
24 \\
39 \\
26\end{array}$ & $\begin{array}{r}9.6 \\
10.5 \\
12.0 \\
16.1 \\
16.1\end{array}$ & $\begin{array}{l}21 \\
12.0 \\
7.7 \\
6.8 \\
6.8\end{array}$ & $\begin{array}{l}4.1 \\
4.0 \\
3.8 \\
3.8 \\
3.8\end{array}$ & $\begin{array}{l}64 \\
60 \\
56 \\
56 \\
41\end{array}$ & $\begin{array}{l}22 \\
32 \\
19.8 \\
24 \\
21\end{array}$ & $\begin{array}{l}12.6 \\
11.6 \\
10.6 \\
10.5 \\
34\end{array}$ & $\begin{array}{l}43 \\
38 \\
24 \\
18.7 \\
34\end{array}$ & $\begin{array}{l}31 \\
30 \\
34 \\
22 \\
19.8\end{array}$ & $\begin{array}{l}38 \\
49 \\
38 \\
25 \\
51\end{array}$ & $\begin{array}{r}10.5 \\
8.3 \\
7.1 \\
9.4 \\
16.1\end{array}$ & $\begin{array}{r}18.4 \\
24 \\
17.1 \\
9.9 \\
8.8\end{array}$ \\
\hline $\begin{array}{l}16 \ldots \\
17 \ldots \\
18 \\
20\end{array}$ & $\begin{array}{l}46 \\
56 \\
40 \\
22 \\
16.1\end{array}$ & $\begin{array}{r}12.9 \\
9.2 \\
13.1 \\
28 \\
11.4\end{array}$ & $\begin{array}{l}7.4 \\
6.9 \\
6.5 \\
5.8 \\
5.8\end{array}$ & $\begin{array}{l}3.7 \\
3.8 \\
5.6 \\
5.1 \\
4.4\end{array}$ & $\begin{array}{l}40 \\
52 \\
56 \\
38 \\
28\end{array}$ & $\begin{array}{l}16.1 \\
17.4 \\
14.2 \\
12.6 \\
11.8\end{array}$ & $\begin{array}{l}53 \\
31 \\
35 \\
27 \\
17.4\end{array}$ & $\begin{array}{l}53 \\
53 \\
34 \\
48 \\
43\end{array}$ & $\begin{array}{l}18.7 \\
17.4 \\
16.1 \\
15.4 \\
15.4\end{array}$ & $\begin{array}{l}27 \\
17.4 \\
14.7 \\
21 \\
24\end{array}$ & $\begin{array}{l}9.7 \\
7.3 \\
6.5 \\
6.0 \\
5.8\end{array}$ & $\begin{array}{l}8 . \\
7 . \\
8 . \\
7 . \\
8 .\end{array}$ \\
\hline 24 & $\begin{array}{l}13.7 \\
12.0 \\
28 \\
15.6 \\
14.4\end{array}$ & $\begin{array}{r}11.3 \\
15.8 \\
8.6 \\
7.4 \\
7.1\end{array}$ & $\begin{array}{l}6.2 \\
5.8 \\
5.2 \\
4.8 \\
4.5\end{array}$ & $\begin{array}{l}3.8 \\
4.0 \\
6.9 \\
5.0 \\
4.1\end{array}$ & $\begin{array}{l}26 \\
53 \\
28 \\
21 \\
18.7\end{array}$ & $\begin{array}{r}11.2 \\
10.6 \\
9.0 \\
9.9 \\
48\end{array}$ & $\begin{array}{l}23 \\
41 \\
46 \\
26 \\
18.7\end{array}$ & $\begin{array}{l}35 \\
49 \\
32 \\
22 \\
19.6\end{array}$ & $\begin{array}{l}36 \\
26 \\
17.4 \\
14.7 \\
13.9\end{array}$ & \begin{tabular}{l|l}
13.9 \\
12.0 \\
11.2 \\
10.6 \\
10.1
\end{tabular} & $\begin{array}{l}5.6 \\
5.6 \\
7.1 \\
6.5 \\
5.7\end{array}$ & $\begin{array}{l}6.3 \\
5.6 \\
5.2 \\
5.0 \\
6.4\end{array}$ \\
\hline $\begin{array}{l}26 \\
27 \\
28 \\
29 \\
30 \\
31 \\
31\end{array}$ & $\begin{array}{l}17.5 \\
53 \\
47 \\
19.8 \\
13.9 \\
34\end{array}$ & $\begin{array}{l}7.6 \\
7.3 \\
6.5 \\
6.3 \\
6.3 \\
6.0\end{array}$ & $\begin{array}{r}4.5 \\
4.2 \\
4.4 \\
9.3 \\
10.5\end{array}$ & \begin{tabular}{r|r}
3.7 & \\
3.4 & \\
3.4 & \\
9.1 & \\
11.2 & \\
7.6 &
\end{tabular} & $\begin{array}{l}17.4 \\
15.9 \\
37 \\
26 \\
18.7 \\
-\end{array}$ & $\begin{array}{l}31 \\
15.6 \\
13.9 \\
21 \\
16.1 \\
12.2\end{array}$ & \begin{tabular}{l|l}
16.1 & \\
17.2 & \\
47 & \\
24 & \\
18.7 & - \\
16.1 & -
\end{tabular} & \begin{tabular}{l}
32 \\
24 \\
27 \\
\hdashline \\
\hdashline \\
\end{tabular} & $\begin{array}{l}13.7 \\
12.9 \\
12.6 \\
22 \\
18.7 \\
13.3\end{array}$ & $\begin{array}{l}9.7 \\
9.0 \\
8.8 \\
8.6 \\
8.4\end{array}$ & $\begin{array}{l}5.4 \\
6.7 \\
12.4 \\
19.9 \\
30 \\
35\end{array}$ & $\begin{array}{l}9.7 \\
7.9 \\
5.7 \\
5.0 \\
4.7\end{array}$ \\
\hline \multirow{2}{*}{\multicolumn{4}{|c|}{ Month }} & \multicolumn{4}{|c|}{ Million gallons a day } & \multirow{2}{*}{\multicolumn{2}{|c|}{ 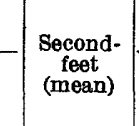 }} & \multicolumn{3}{|c|}{ Total run-off } \\
\hline & & & & Aaximum & Min & mum & Mean & & & $\begin{array}{l}\text { Million } \\
\text { gallons }\end{array}$ & \multicolumn{2}{|c|}{ Acre-feet } \\
\hline \multicolumn{4}{|c|}{$\begin{array}{l}\text { July } \\
\text { August } \\
\text { September } \\
\text { October } \\
\text { November } \\
\text { December } \\
\text { January } \\
\text { February } \\
\text { March } \\
\text { April } \\
\text { May }\end{array}$} & \begin{tabular}{l|}
56 \\
45 \\
29 \\
11.8 \\
64 \\
48 \\
53 \\
53 \\
60 \\
51 \\
35 \\
39
\end{tabular} & & $\begin{array}{r}8.1 \\
6.0 \\
4.2 \\
3.4 \\
4.4 \\
9.0 \\
10.5 \\
10.5 \\
12.6 \\
8.4 \\
5.4 \\
4.7\end{array}$ & $\begin{array}{l}26.7 \\
12.6 \\
10.4 \\
5.43 \\
38.7 \\
22.0 \\
24.7 \\
26.8 \\
28.3 \\
18.3 \\
10.7 \\
11.1\end{array}$ & \multicolumn{2}{|c|}{$\begin{array}{l}41.3 \\
19.5 \\
16.1 \\
8.40 \\
59.9 \\
34.0 \\
38.2 \\
41.5 \\
43.8 \\
28.3 \\
16.6 \\
17.2\end{array}$} & $\begin{array}{r}828 \\
389 \\
313 \\
168 \\
1,160 \\
682 \\
765 \\
751 \\
877 \\
549 \\
332 \\
332\end{array}$ & \multicolumn{2}{|r|}{$\begin{array}{r}2,540 \\
1,200 \\
957 \\
517 \\
3,560 \\
2,090 \\
2,350 \\
2,300 \\
2,690 \\
1,680 \\
1,020 \\
1,020\end{array}$} \\
\hline \multicolumn{4}{|l|}{ The year.. } & 64 & & 3.4 & 19.6 & \multicolumn{2}{|c|}{30.3} & 7,150 & \multicolumn{2}{|r|}{21,900} \\
\hline
\end{tabular}


WATAHULU STREAM ABOVE KOATE STREAM, MEAR WATMRA, KAUAT

Location.-Water-stage recorder in Waimea Canyon, half a mile above confluence with Koaie Stream and $8 \% 4$ miles north of Waimea.

Drainage area.-20.0 square miles.

ReCoRDS AVAILABLE. - February to October, 1916; October, 1917, to June, 1918; and May, 1925, to June, 1929.

Extremes.- Maximum discharge during year, 2,310 million gallons a day or 3,570 second-feet Nov. 4 (gage height, 9.33 feet); minimum, 7.2 million gallons a day or 11.1 second-feet June 10 .

1915-1918, 1925-1929: Maximum discharge, 2,550 million gallons a day or 3,950 second-feet Dec. 24, 1927 (gage height, 9.92 feet); minimum, 52. million gallons a day or 8.0 second-feet Nov. 4,1927 .

REMARKs.- Records good for ordinary stages; poor for estimated periods and all high-stage records. Kokee ditch diverts water for irrigation above station.

Discharge, in million gallons a day, 1928-29

\begin{tabular}{|c|c|c|c|c|c|c|c|c|c|c|c|c|}
\hline Day & July & Aug. & Sept. & Oct. & Nov. & Dec. & Jan. & Feb. & Mar. & Apr. & May & June \\
\hline $\begin{array}{l}1 \ldots \\
2 \ldots \\
3-.- \\
4 \ldots \\
5 \ldots\end{array}$ & $\begin{array}{l}13.0 \\
14.8 \\
11.4 \\
10.8 \\
10.8\end{array}$ & $\begin{array}{r}12.9 \\
12.3 \\
10.6 \\
9.6 \\
9.4\end{array}$ & $\begin{array}{r}8.2 \\
8.9 \\
9.6 \\
34 \\
18.5\end{array}$ & & $\begin{array}{l}8.0 \\
7.8 \\
7.8\end{array}$ & 12 & $\begin{array}{l}10 \\
34\end{array}$ & $\begin{array}{l}11.2 \\
10.9 \\
10.6 \\
10.6 \\
10.6\end{array}$ & $\begin{array}{l}17.4 \\
14.2 \\
84 \\
47 \\
207\end{array}$ & $\begin{array}{l}10.9 \\
10.6 \\
10.4 \\
10.4 \\
10.4\end{array}$ & $\begin{array}{r}11.8 \\
9.9 \\
9.9 \\
9.9 \\
9.9\end{array}$ & $\begin{array}{r}37 \\
12.9 \\
9.9 \\
8.7 \\
9.6\end{array}$ \\
\hline $\begin{array}{r}6 \\
7 \\
8 \\
9 \\
10\end{array}$ & \begin{tabular}{r|}
11.1 \\
14.8 \\
209 \\
17.6 \\
13.2
\end{tabular} & $\begin{array}{l}9.4 \\
9.6 \\
9.4 \\
9.2 \\
8.9\end{array}$ & $\begin{array}{r}10.1 \\
9.2 \\
10.1 \\
9.9 \\
9.2\end{array}$ & 9.5 & 300 & 32 & 13 & $\begin{array}{l}10.4 \\
10.4 \\
10.6 \\
10.6 \\
13.1\end{array}$ & $\begin{array}{r}41 \\
110 \\
94 \\
32 \\
24\end{array}$ & $\begin{array}{l}10.4 \\
10.4 \\
10.4 \\
10.4 \\
64\end{array}$ & $\begin{array}{r}9.9 \\
9.9 \\
10.9 \\
11.5 \\
10.9\end{array}$ & $\begin{array}{l}9.9 \\
8.4 \\
7.6 \\
7.4 \\
7.4\end{array}$ \\
\hline $\begin{array}{l}11 \\
12 \\
15\end{array}$ & $\begin{array}{l}53 \\
17.2 \\
13.8 \\
29 \\
13.2\end{array}$ & $\begin{array}{l}8.9 \\
8.9 \\
8.9 \\
9.6 \\
9.9\end{array}$ & $\begin{array}{r}13.6 \\
11.8 \\
9.9 \\
9.2 \\
9.2\end{array}$ & & & & & $\begin{array}{l}192 \\
34 \\
22 \\
16.6 \\
83\end{array}$ & $\begin{array}{l}17.4 \\
14.6 \\
14.2 \\
13.2 \\
12.6\end{array}$ & $\begin{array}{l}21 \\
85 \\
18.2 \\
64 \\
41\end{array}$ & $\begin{array}{l}10.6 \\
10.6 \\
10.6 \\
10.9 \\
11.2\end{array}$ & $\begin{array}{l}7.4 \\
8.2 \\
8.0 \\
7.8 \\
8.0\end{array}$ \\
\hline $\begin{array}{l}16 \\
18 \\
18\end{array}$ & $\begin{array}{l}69 \\
200 \\
26 \\
12.3 \\
10.6\end{array}$ & $\begin{array}{r}9.6 \\
9.2 \\
9.9 \\
17.4 \\
11.2\end{array}$ & $\begin{array}{l}9.2 \\
9.2 \\
9.2 \\
9.4 \\
9.4\end{array}$ & $\begin{array}{l}8.7 \\
8.7 \\
8.7\end{array}$ & & 11 & 30 & $\begin{array}{r}228 \\
97 \\
27 \\
45 \\
28\end{array}$ & $\begin{array}{l}12.3 \\
12.0 \\
11.8 \\
11.5 \\
12.0\end{array}$ & $\begin{array}{l}20 \\
13.5 \\
12.6 \\
21 \\
15.2\end{array}$ & $\begin{array}{l}11.2 \\
11.5 \\
11.5 \\
11.5 \\
11.5\end{array}$ & $\begin{array}{l}8.0 \\
8.2 \\
8.4 \\
8.7 \\
8.9\end{array}$ \\
\hline $\begin{array}{l}21 \\
22 \\
25\end{array}$ & $\begin{array}{r}10.1 \\
10.1 \\
12.3 \\
10.4 \\
9.9\end{array}$ & $\begin{array}{r}10.1 \\
9.4 \\
9.2 \\
8.7 \\
8.4\end{array}$ & $\begin{array}{l}9.9 \\
9.6 \\
9.2 \\
9.2 \\
9.2\end{array}$ & $\begin{array}{l}8.7 \\
8.7 \\
8.7 \\
8.7 \\
8.7\end{array}$ & 20 & & & $\begin{array}{l}26 \\
78 \\
24 \\
17.0 \\
15.6\end{array}$ & $\begin{array}{l}73 \\
19.0 \\
12.9 \\
12.0 \\
11.5\end{array}$ & $\begin{array}{l}13.5 \\
13.2 \\
12.9 \\
12.9 \\
12.9\end{array}$ & $\begin{array}{l}11.5 \\
11.5 \\
11.5 \\
11.5 \\
11.2\end{array}$ & $\begin{array}{r}9.2 \\
9.4 \\
9.6 \\
9.9 \\
10.4\end{array}$ \\
\hline $\begin{array}{l}26 \\
28 \\
28 \\
30 \\
31\end{array}$ & $\begin{array}{c}9.9 \\
107 \\
52 \\
12.9 \\
10.4 \\
10.1\end{array}$ & $\begin{array}{l}8.4 \\
8.4 \\
8.4 \\
8.4 \\
8.2 \\
8.2\end{array}$ & $\begin{array}{l}9.2 \\
9.2 \\
9.2 \\
9.4 \\
9.6\end{array}$ & $\begin{array}{l}8.7 \\
8.7 \\
8.7 \\
9.6 \\
8.4 \\
8.0\end{array}$ & & 22 & $\begin{array}{l}96 \\
17.0 \\
13.2 \\
11.8\end{array}$ & \begin{tabular}{c}
21 \\
16.3 \\
16.0 \\
\hdashline.- \\
\\
\hdashline
\end{tabular} & $\begin{array}{l}12.3 \\
11.2 \\
11.5 \\
14.6 \\
12.6 \\
11.5\end{array}$ & $\begin{array}{l}12.6 \\
12.6 \\
12.3 \\
12.0 \\
12.0\end{array}$ & $\begin{array}{l}10.9 \\
11.2 \\
12.0 \\
26 \\
26 \\
39\end{array}$ & $\begin{array}{l}9.4 \\
8.2 \\
8.0 \\
8.0 \\
8.0\end{array}$ \\
\hline
\end{tabular}

\begin{tabular}{|c|c|c|c|c|c|c|}
\hline \multirow{2}{*}{ Month } & \multicolumn{3}{|c|}{ Million gallons a day } & \multirow{2}{*}{$\begin{array}{l}\text { Second- } \\
\text { feet } \\
\text { (mean) }\end{array}$} & \multicolumn{2}{|c|}{ Total run-off } \\
\hline & Maximum & Minimum & Mean & & $\begin{array}{l}\text { Millon } \\
\text { gallons }\end{array}$ & Acre-feet \\
\hline $\begin{array}{l}\text { July } \\
\text { August } \\
\text { September } \\
\text { October } \\
\text { November } \\
\text { December } \\
\text { January } \\
\text { February } \\
\text { March } \\
\text { April } \\
\text { May } \\
\text { June }\end{array}$ & \begin{tabular}{|c|}
209 \\
17.4 \\
34
\end{tabular} \mid $\begin{array}{c}28 \\
207 \\
85 \\
39 \\
37\end{array}$ & \begin{tabular}{r|r|}
9.9 \\
8.2 \\
8.2 \\
10.4 \\
11.2 \\
10.4 \\
9.9 \\
7.4
\end{tabular} & $\begin{array}{c}33.1 \\
9.70 \\
10.7 \\
9.14 \\
99.1 \\
19.0 \\
22.7 \\
39.1 \\
32.4 \\
19.9 \\
12.8 \\
9.68\end{array}$ & $\begin{array}{r}51.2 \\
15.0 \\
16.6 \\
14.1 \\
153 \\
29.4 \\
35.1 \\
60.5 \\
50.1 \\
30.8 \\
19.8 \\
15.0\end{array}$ & $\begin{array}{r}1,030 \\
301 \\
322 \\
283 \\
2,970 \\
590 \\
704 \\
1,100 \\
1,000 \\
597 \\
398 \\
290\end{array}$ & $\begin{array}{r}3,150 \\
923 \\
985 \\
870 \\
9,120 \\
1,810 \\
2,160 \\
3,360 \\
3,080 \\
1,830 \\
1,220 \\
891\end{array}$ \\
\hline The year. & 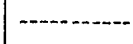 & - & 26.3 & 40.7 & 9,580 & 29,400 \\
\hline
\end{tabular}




\section{KOATE STREAM AT ELEVATION 3,700 FEET, NBAR WAIMEA, KAUAI}

Location.-Water-stage recorder at elevation 3,700 feet, $12 \frac{132}{2}$ miles N. $30^{\circ}$ E. from Waimea.

Drainage Area. - 3.4 square miles.

Records availabre. -July, 1919, to June, 1929.

EXTREmes.-Maximum discharge during year, 1,040 million gallons a day or 1,610 second-feet Nov. 4 (gage height, 2.99 feet); minimum, 0.6 million gallons a day or 0.9 second-foot May 21 and 22.

1919-1929: Maximum discharge, about 3,750 million gallons a day or 5,800 second-feet Jan. 16, 1921 (gage height, 6.70 feet); minimum, that of May 21 and 22, 1929.

REMARKs.- Records good except those for high stages, which are poor. No diversions.

Discharge, in million gallons a day, 1928-29

\begin{tabular}{|c|c|c|c|c|c|c|c|c|c|c|c|c|}
\hline Day & July & Aug. & Sept. & Oct. & Nov. & Dec. & Jan. & Feb. & Mar. & Apr. & May & June \\
\hline $\begin{array}{l}1 \ldots \\
2 \ldots \\
3 \\
4 \ldots \\
5\end{array}$ & $\begin{array}{l}74 \\
14.7 \\
6.1 \\
11.6 \\
24\end{array}$ & $\begin{array}{r}14.2 \\
12.5 \\
8.1 \\
7.0 \\
5.7\end{array}$ & $\begin{array}{l}a 4.9 \\
14 \\
69 \\
14.4\end{array}$ & $\begin{array}{r}4.4 \\
83 \\
8.2 \\
4.5 \\
6.1\end{array}$ & $\begin{array}{r}2.5 \\
1.8 \\
1.8 \\
372 \\
24\end{array}$ & $\begin{array}{r}4.5 \\
3.5 \\
3.2 \\
2.9 \\
24\end{array}$ & $\begin{array}{r}2.7 \\
2.5 \\
18.0 \\
26 \\
6.6\end{array}$ & $\begin{array}{l}2.1 \\
1.9 \\
1.6 \\
1.5 \\
1.5\end{array}$ & $\begin{array}{c}12.1 \\
5.7 \\
41 \\
22 \\
74\end{array}$ & $\begin{array}{l}1.9 \\
1,8 \\
1.6 \\
1.5 \\
1.9\end{array}$ & $\begin{array}{l}1.5 \\
1.3 \\
1.5 \\
1.3 \\
1.5\end{array}$ & $\begin{array}{r}63 \\
4.8 \\
3.8 \\
3.2 \\
10.6\end{array}$ \\
\hline $\begin{array}{r}6 \\
7 \\
8 \\
8 \\
10\end{array}$ & $\begin{array}{l}8.1 \\
18.1 \\
87 \\
12.2 \\
15.3\end{array}$ & $\begin{array}{l}\mathbf{5 . 3} \\
5.3 \\
6.5 \\
4.9 \\
4.2\end{array}$ & $\begin{array}{r}5.7 \\
6.9 \\
14.3 \\
19.0 \\
46\end{array}$ & $\begin{array}{l}6.6 \\
4.2 \\
3.8 \\
3.5 \\
3.5\end{array}$ & $\begin{array}{r}157 \\
102 \\
58 \\
101 \\
141\end{array}$ & $\begin{array}{l}24 \\
12.0 \\
19.8 \\
29\end{array}$ & $\begin{array}{l}3.8 \\
2.9 \\
3.5 \\
2.9 \\
2.3\end{array}$ & $\begin{array}{l}1.3 \\
1.2 \\
1.1 \\
1.1 \\
1.3\end{array}$ & $\begin{array}{l}26 \\
47 \\
46 \\
35 \\
27\end{array}$ & $\begin{array}{r}1.9 \\
1.6 \\
1.3 \\
1.3 \\
25\end{array}$ & $\begin{array}{l}1.9 \\
2.1 \\
5.2 \\
3.2 \\
2.1\end{array}$ & $\begin{array}{l}5.7 \\
3.2 \\
2.1 \\
1.6 \\
1.5\end{array}$ \\
\hline $\begin{array}{l}11 \ldots \\
12 . . \\
13 \\
14 \\
15 . .\end{array}$ & $\begin{array}{l}79 \\
18.8 \\
14.2 \\
25 \\
12.7\end{array}$ & $\begin{array}{r}3.9 \\
5.3 \\
18.7 \\
17.6 \\
7.0\end{array}$ & $\begin{array}{c}85 \\
9.1 \\
5.7 \\
4.9 \\
7.0\end{array}$ & $\begin{array}{l}3.2 \\
2.9 \\
2.5 \\
2.3 \\
2.3\end{array}$ & $\begin{array}{l}91 \\
17.7 \\
13.7 \\
22 \\
14.6\end{array}$ & $\begin{array}{r}17 \\
\\
\text { a } 8.3 \\
7.1\end{array}$ & $\begin{array}{r}2.1 \\
1.8 \\
1.6 \\
1.8 \\
25\end{array}$ & \begin{tabular}{|c|}
14.2 \\
54 \\
21 \\
22 \\
145
\end{tabular} & $\begin{array}{l}8.3 \\
4.8 \\
3.8 \\
3.2 \\
2.7\end{array}$ & $\begin{array}{c}26 \\
32 \\
24 \\
6.6 \\
36\end{array}$ & $\begin{array}{l}1.6 \\
1.3 \\
1.1 \\
1.1 \\
1.1\end{array}$ & $\begin{array}{l}1.5 \\
2.1 \\
3.2 \\
3.2 \\
2.7\end{array}$ \\
\hline $\begin{array}{l}16 \\
17 \\
18 \\
19 \\
20\end{array}$ & $\begin{array}{r}34 \\
97 \\
14.8 \\
7.5 \\
6.1\end{array}$ & $\begin{array}{l}6.5 \\
25 \\
50 \\
39 \\
8.1\end{array}$ & $\begin{array}{r}7.0 \\
9.9 \\
13.0 \\
7.0 \\
16.2\end{array}$ & $\begin{array}{l}2.3 \\
3.4 \\
4.8 \\
3.8 \\
3.5\end{array}$ & $\begin{array}{l}17.7 \\
23 \\
40 \\
15.6 \\
8.3\end{array}$ & $\begin{array}{r}11.4 \\
7.8 \\
4.5 \\
3.5 \\
2.9\end{array}$ & $\begin{array}{l}27 \\
15.0 \\
23 \\
8.2 \\
4.2\end{array}$ & \begin{tabular}{|l|}
133 \\
43 \\
12.2 \\
19.6 \\
28
\end{tabular} & $\begin{array}{l}2.3 \\
2.1 \\
1.8 \\
1.5 \\
4.4\end{array}$ & $\begin{array}{r}11.0 \\
5.2 \\
3.8 \\
30 \\
16.4\end{array}$ & $\begin{array}{r}1.0 \\
.9 \\
.8 \\
.8 \\
.7\end{array}$ & $\begin{array}{l}2.3 \\
2.3 \\
2.1 \\
1.6 \\
1.2\end{array}$ \\
\hline $\begin{array}{l}21 \\
22 \\
23 \\
25\end{array}$ & $\begin{array}{r}4.9 \\
5.2 \\
17.4 \\
7.0 \\
5.3\end{array}$ & $\begin{array}{r}6.1 \\
12.6 \\
6.5 \\
4.9 \\
4.5\end{array}$ & $\begin{array}{l}9.9 \\
5.7 \\
4.2 \\
3.6 \\
3.3\end{array}$ & $\begin{array}{l}2.7 \\
2.9 \\
4.5 \\
2.5 \\
1.9\end{array}$ & $\begin{array}{r}6.6 \\
14.6 \\
8.3 \\
5.2 \\
4.2\end{array}$ & $\begin{array}{r}2.7 \\
2.3 \\
2.1 \\
2.1 \\
33\end{array}$ & $\begin{array}{l}5.0 \\
24 \\
55 \\
7.0 \\
4.2\end{array}$ & $\begin{array}{c}14.3 \\
29 \\
8.3 \\
4.8 \\
3.5\end{array}$ & $\begin{array}{l}79 \\
8.1 \\
3.8 \\
2.7 \\
4.5\end{array}$ & $\begin{array}{l}4.8 \\
3.5 \\
2.9 \\
2.5 \\
2.1\end{array}$ & $\begin{array}{r}.7 \\
.7 \\
1.0 \\
.8 \\
.9\end{array}$ & $\begin{array}{r}1.0 \\
.8 \\
.8 \\
.7 \\
.8\end{array}$ \\
\hline 30 28 & $\begin{array}{l}5.3 \\
62 \\
86 \\
9.8 \\
6.5 \\
8.6\end{array}$ & $\begin{array}{l}5.7 \\
6.1 \\
4.5 \\
\text { 4. } 5 \\
4.2 \\
3.9\end{array}$ & $\begin{array}{l}3.0 \\
2.8 \\
7.0 \\
9.1 \\
6.1\end{array}$ & $\begin{array}{r}1.6 \\
1.6 \\
1.6 \\
15.2 \\
6.2 \\
4.2\end{array}$ & $\begin{array}{r}3.2 \\
2.9 \\
18.6 \\
8.9 \\
5.2\end{array}$ & $\begin{array}{r}9.9 \\
4.8 \\
5.7 \\
31 \\
6.1 \\
3.6\end{array}$ & $\begin{array}{l}3.2 \\
37 \\
29 \\
5.2 \\
3.2 \\
2.5\end{array}$ & \begin{tabular}{r}
9.3 \\
4.5 \\
16.0 \\
\hdashline-9 \\
\hdashline-9 \\
\end{tabular} & $\begin{array}{l}3.8 \\
2.5 \\
2.5 \\
6.9 \\
3.2 \\
2.3\end{array}$ & $\begin{array}{l}1.9 \\
1.8 \\
1.6 \\
1.5 \\
1.3\end{array}$ & $\begin{array}{r}1.1 \\
18.0 \\
11.1 \\
3.8 \\
8.2 \\
17.0\end{array}$ & $\begin{array}{r}.8 \\
.7 \\
.7 \\
.9 \\
1.2\end{array}$ \\
\hline
\end{tabular}

\begin{tabular}{|c|c|c|c|c|c|c|}
\hline \multirow{2}{*}{ Month } & \multicolumn{3}{|c|}{ - Million gallons a day } & \multirow{2}{*}{$\begin{array}{l}\text { Second- } \\
\text { feet } \\
\text { (mean) }\end{array}$} & \multicolumn{2}{|c|}{ Total run-off } \\
\hline & Maximum & Minimum & Mean & & $\begin{array}{l}\text { Million } \\
\text { gallons }\end{array}$ & Acre-feet \\
\hline $\begin{array}{l}\text { July } \\
\text { August } \\
\text { September } \\
\text { October } \\
\text { November } \\
\text { December. } \\
\text { January } \\
\text { February } \\
\text { March } \\
\text { April } \\
\text { May } \\
\text { June. }\end{array}$ & $\begin{array}{r}97 \\
50 \\
85 \\
83 \\
372 \\
33 \\
55 \\
145 \\
79 \\
36 \\
18.0 \\
63\end{array}$ & $\begin{array}{l}4.9 \\
3.9 \\
2.8 \\
1.6 \\
1.8 \\
2.1 \\
1.6 \\
1.1 \\
1.5 \\
1.3 \\
.7 \\
.7\end{array}$ & $\begin{array}{c}25.7 \\
10.3 \\
14.3 \\
6.57 \\
43.4 \\
11.0 \\
11.5 \\
21.3 \\
15.8 \\
8.49 \\
3.07 \\
4.34\end{array}$ & $\begin{array}{c}39.8 \\
15.9 \\
22.1 \\
10.2 \\
67.1 \\
17.0 \\
17.8 \\
33.0 \\
24.4 \\
13.1 \\
4.75 \\
6.71\end{array}$ & $\begin{array}{c}798 \\
318 \\
428 \\
204 \\
1,300 \\
340 \\
356 \\
596 \\
490 \\
255 \\
95.3 \\
130\end{array}$ & $\begin{array}{r}2,440 \\
980 \\
1,320 \\
625 \\
4,000 \\
1,050 \\
1,090 \\
1,830 \\
1,500 \\
782 \\
292 \\
400\end{array}$ \\
\hline The year... & 372 & .7 & 14.6 & 22.6 & 5,310 & 16,300 \\
\hline
\end{tabular}

- Partly estimated. 
WAIALAE RIVRR AT ELEVATION 3,700 FEET, INEAR WATMLA, KAUAI

Location.-Water-stage recorder at elevation 3,700 feet, $101 / 2$ miles N. $30^{\circ} \mathrm{E}$. from Waimea.

Drainage area.-3.3 square miles.

Records Available. - January, 1920, to June, 1929. August, 1910, to January, 1916 , at site 2 miles downstream.

Extremes.-Maximum discharge during year, 984 million gallons a day or 1,520 second-feet Nov. 4 (gage height, 3.98 feet); minimum, 1.2 million gallons a day or 1.9 second-feet June 30 .

1920-1929: Maximum discharge (estimated), 4,500 million gallons a day or 6,960 second-feet Jan. 16, 1921 (gage height, 8.44 feet); minimum, 0.7 million gallons a day or 1.1 second-feet Mar. 18-20, 1925.

Remarks.-Records good for ordinary stages, fair for estimated periods, and poor for extremely high and low stages. No diversions.

Discharge, in million gallons a day, 1928-29

\begin{tabular}{|c|c|c|c|c|c|c|c|c|c|c|c|c|}
\hline Day & July & Aug. & Sept. & Oct. I & Nov. & Dec. & Jan. & Feb. & Mar. & A pr. & May & June \\
\hline & $\begin{aligned} 59 \\
7.8 \\
2.8 \\
7.5 \\
20\end{aligned}$ & $\begin{array}{l}7.4 \\
6.8 \\
4.6 \\
3.9 \\
3.2\end{array}$ & $\begin{array}{c}2.8 \\
6.2 \\
22 \\
9.5\end{array}$ & \begin{tabular}{c|c}
2.8 & \\
75 & \\
7.1 & \\
4.1 & 3 \\
4.3 & 1
\end{tabular} & \begin{tabular}{r|}
3.5 \\
3.0 \\
2.8 \\
362 \\
182
\end{tabular} & \begin{tabular}{r|}
4.3 \\
3.7 \\
3.5 \\
3.0 \\
11.3
\end{tabular} & \begin{tabular}{r|}
2.8 \\
2.8 \\
8.3 \\
16.6 \\
4.6
\end{tabular} & $\begin{array}{l}3.0 \\
2.8 \\
2.8 \\
2.8 \\
2.8\end{array}$ & $\begin{array}{l}11.0 \\
7.4 \\
21 \\
14.9 \\
41\end{array}$ & $\begin{array}{l}3.1 \\
2.9 \\
2.9 \\
2.9 \\
3.4\end{array}$ & $\begin{array}{l}2.4 \\
2.4 \\
2.9\end{array}$ & 10. \\
\hline $\begin{array}{c}6 \\
7 \\
8 \\
9\end{array}$ & $\begin{array}{r}4.8 \\
13.3 \\
49 \\
5.9 \\
\times 19.4\end{array}$ & $\begin{array}{l}2.8 \\
2.6 \\
3.5 \\
2.6 \\
2.5\end{array}$ & $\begin{array}{r}3.9 \\
3.9 \\
7.9 \\
15.4 \\
37\end{array}$ & \begin{tabular}{l|l}
4.6 & 1 \\
3.5 & \\
3.0 & \\
3.2 & \\
3.7 & 1
\end{tabular} & $\begin{array}{r}138 \\
90 \\
41 \\
68 \\
118\end{array}$ & 16 & $\begin{array}{l}3.2 \\
2.8 \\
2.8 \\
2.8 \\
2.6\end{array}$ & $\begin{array}{l}2.8 \\
2.8 \\
2.6 \\
2.6 \\
2.8\end{array}$ & $\begin{array}{l}17.5 \\
29 \\
30 \\
24 \\
20\end{array}$ & $\begin{array}{r}2.9 \\
2.9 \\
2.8 \\
2.8 \\
16.9\end{array}$ & $\begin{array}{l}3.4 \\
2.8\end{array}$ & 3 \\
\hline (1) & $\begin{array}{c}74 \\
18.6 \\
11.2 \\
24 \\
8.0\end{array}$ & $\begin{array}{r}2.2 \\
2.4 \\
10.8 \\
12.9 \\
4.8\end{array}$ & $\begin{array}{l}63 \\
6.1 \\
3.9 \\
3.2 \\
4.1\end{array}$ & $\begin{array}{l}3.0 \\
2.5 \\
2.5 \\
2.4 \\
2.4\end{array}$ & \begin{tabular}{l|}
74 \\
15.3 \\
10.5 \\
17.0 \\
11.0
\end{tabular} & 5 & $\begin{array}{r}2.5 \\
2.4 \\
2.4 \\
2.4 \\
14.3\end{array}$ & $\begin{array}{l}13.8 \\
54 \\
21 \\
28 \\
162\end{array}$ & $\begin{array}{l}8.0 \\
5.6 \\
4.8 \\
4.4 \\
4.4\end{array}$ & $\begin{array}{c}12.8 \\
21 \\
9.8 \\
5.4 \\
26\end{array}$ & $\begin{array}{l}2.0 \\
2.4\end{array}$ & 3. \\
\hline 6. & $\begin{aligned} 28 \\
74 \\
11.5 \\
5.6 \\
4.3\end{aligned}$ & $\begin{array}{l}4.1 \\
28 \\
24 \\
28 \\
4.8\end{array}$ & $\begin{array}{r}4.1 \\
5.4 \\
7.0 \\
4.3 \\
11.7\end{array}$ & \begin{tabular}{l|}
2.4 \\
3.4 \\
3.9 \\
3.2 \\
3.2
\end{tabular} & $\begin{array}{c}11.8 \\
9.5 \\
24 \\
8.8 \\
5.3\end{array}$ & $\begin{array}{l}6.7 \\
5.6 \\
3.9 \\
3.2 \\
2.8\end{array}$ & $\begin{array}{c}15.7 \\
13.1 \\
24 \\
6.4 \\
3.9\end{array}$ & $\begin{array}{l}189 \\
40 \\
13.2 \\
13.2 \\
21\end{array}$ & $\begin{array}{r}3.8 \\
3.4 \\
3.1 \\
2.9 \\
14.6\end{array}$ & $\begin{array}{r}6.0 \\
3.8 \\
3.1 \\
27 \\
7.9\end{array}$ & $\begin{array}{l}2.4 \\
2.2 \\
2.2\end{array}$ & 3. \\
\hline (19 & $\begin{array}{l}3.5 \\
3.2 \\
5.6 \\
4.1 \\
3.2\end{array}$ & $\begin{array}{l}4.1 \\
7.4 \\
4.3 \\
3.0 \\
2.6\end{array}$ & $\begin{array}{l}6.6 \\
3.9 \\
2.8 \\
2.5 \\
2.4\end{array}$ & $\begin{array}{l}3.0 \\
3.5 \\
4.3 \\
3.0 \\
2.8\end{array}$ & $\begin{array}{l}4.6 \\
6.5 \\
5.0 \\
3.9 \\
3.2\end{array}$ & $\begin{array}{r}2.6 \\
2.5 \\
2.4 \\
2.4 \\
14.0\end{array}$ & \begin{tabular}{c|}
3.5 \\
23 \\
35 \\
5.6 \\
3.9
\end{tabular} & $\begin{array}{r}12.1 \\
18.4 \\
8.3 \\
5.9 \\
6.2\end{array}$ & $\begin{array}{c}75 \\
9.6 \\
5.6 \\
4.4 \\
5.1\end{array}$ & $\begin{array}{l}4.1 \\
3.1 \\
2.8 \\
2.8 \\
2.6\end{array}$ & $\begin{array}{l}2.2 \\
2.2 \\
2.2 \\
2.2 \\
2.1\end{array}$ & $\begin{array}{l}2 . \\
2 . \\
2 . \\
2 .\end{array}$ \\
\hline 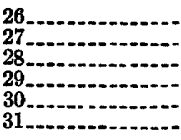 & $\begin{array}{c}2.8 \\
38 \\
87 \\
7.2 \\
4.6 \\
5.0\end{array}$ & $\begin{array}{l}3.2 \\
3.0 \\
2.6 \\
2.4 \\
2.4 \\
2.2\end{array}$ & $\begin{array}{l}2.2 \\
2.0 \\
6.4 \\
5.9 \\
3.9\end{array}$ & \begin{tabular}{r|r}
2.6 \\
2.5 \\
2.5 \\
22 \\
6.5 \\
4.6 &
\end{tabular} & $\begin{array}{r}3.2 \\
2.8 \\
18.6 \\
9.0 \\
5.0\end{array}$ & $\begin{array}{r}5.9 \\
3.5 \\
6.5 \\
23 \\
4.8 \\
3.1\end{array}$ & $\begin{array}{c}3.2 \\
51 \\
22 \\
5.2 \\
3.9 \\
3.5\end{array}$ & \begin{tabular}{r}
17.2 \\
6.6 \\
14.7 \\
\hdashline... \\
\hdashline..-- \\
..--
\end{tabular} & $\begin{array}{l}4.6 \\
3.6 \\
3.9 \\
7.1 \\
4.1 \\
\text { 3. } 6\end{array}$ & $\begin{array}{l}2.4 \\
2.4 \\
2.4 \\
2.4 \\
2.2\end{array}$ & $\begin{array}{r}2.1 \\
12.2 \\
15.1 \\
9.0 \\
14.4 \\
10.6\end{array}$ & $\begin{array}{l}2 . \\
1 . \\
1 . \\
1 . \\
1 .\end{array}$ \\
\hline \multirow{2}{*}{\multicolumn{4}{|c|}{ Month }} & \multicolumn{4}{|c|}{ Million gallons a day } & \multirow{2}{*}{\multicolumn{2}{|c|}{$\begin{array}{c}\text { Second- } \\
\text { feet } \\
\text { (mean) }\end{array}$}} & \multicolumn{3}{|c|}{ Total run-off } \\
\hline & & & & Laximum & Min & num & Mean & & & $\begin{array}{l}\text { Million } \\
\text { gallons }\end{array}$ & \multicolumn{2}{|c|}{ Acre-feet } \\
\hline $\begin{array}{l}\text { July } \\
\text { Augu } \\
\text { Septe } \\
\text { Octol } \\
\text { Nove } \\
\text { Deces } \\
\text { Janug } \\
\text { Febrr } \\
\text { Marc } \\
\text { April } \\
\text { May }\end{array}$ & 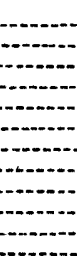 & & & \begin{tabular}{c}
87 \\
28 \\
63 \\
75 \\
362 \\
\hdashline 51 \\
189 \\
75 \\
27 \\
15.1 \\
59
\end{tabular} & & \begin{tabular}{r}
2.8 \\
2.2 \\
2.0 \\
2.4 \\
2.8 \\
\hdashline 2.4 \\
2.6 \\
2.9 \\
2.2 \\
2.1 \\
1.3
\end{tabular} & $\begin{array}{r}19.8 \\
6.42 \\
9.40 \\
6.37 \\
41.9 \\
7.93 \\
9.55 \\
24.0 \\
12.8 \\
6.45 \\
4.09 \\
5.51\end{array}$ & \multicolumn{2}{|c|}{$\begin{array}{c}30.6 \\
9.93 \\
14.5 \\
9.86 \\
64.8 \\
12.3 \\
14.8 \\
37.1 \\
19.8 \\
9.98 \\
6.33 \\
8.53\end{array}$} & $\begin{array}{r}613 \\
199 \\
282 \\
198 \\
1,260 \\
246 \\
296 \\
672 \\
397 \\
194 \\
127 \\
165\end{array}$ & \multicolumn{2}{|r|}{$\begin{array}{r}1,880 \\
611 \\
865 \\
606 \\
3,860 \\
754 \\
909 \\
2,060 \\
1,220 \\
594 \\
389 \\
507\end{array}$} \\
\hline \multicolumn{4}{|c|}{ The year...................... } & 362 & & 1.3 & 12.7 & & 9.6 & 4,650 & & 14,30 \\
\hline
\end{tabular}

a.Partly estimated. 


\section{KEKAHA DITCH AT CAMP NO. 1, NEAR WAMEA, KADAI}

Location.-Water-stage recorder in Waimea Canyon, $6 \frac{1}{4}$ miles N. $16^{\circ}$ E. of Waimea.

Records available.-November, 1907, to June, 1915; March, 1916, to June 1929.

Extremes.-Maximum discharge during year, 66 million gallons a day or 102 second-feet Sept. 10 (gage height, 4.18 feet); no flow several days in August and April.

1907-1929: Maximum discharge, 71 million gallons a day or 110 secondfeet Apr. 25, 1928 (gage height, 4.33 feet); no flow occasionally when water is shut out of the ditch.

REMARKs.- Records good for ordinary stages except those estimated, which are fair; records for extremely low stages poor. Intake on Waimea River 8 miles north of Waimea. Water used for irrigation of sugar cane around Kekaha. Regulated by head gates.

Discharge, in million gallons a day, 1928-29

\begin{tabular}{|c|c|c|c|c|c|c|c|c|c|c|c|c|}
\hline Day & July & Aug. & Sept. & Oct. & Nov. & Dec. & Jan. & b. & Mar. & Apr. & May & June \\
\hline $\begin{array}{l}1 \\
2 \\
3 \\
4 \\
5\end{array}$ & $\begin{array}{l}38 \\
48 \\
36 \\
32 \\
50\end{array}$ & $\begin{array}{l}44 \\
47 \\
36 \\
33 \\
30\end{array}$ & $\begin{array}{l}24 \\
30 \\
44 \\
50 \\
48\end{array}$ & \begin{tabular}{l|r}
26 & 2 \\
42 & 2 \\
47 & 2 \\
33 & 1 \\
29 & $a$
\end{tabular} & $\begin{array}{l}26 \\
24 \\
23 \\
19.5 \\
42\end{array}$ & $\begin{array}{l}33 \\
33 \\
33 \\
33 \\
44\end{array}$ & $\begin{array}{l}26 \\
30 \\
39 \\
44 \\
41\end{array}$ & $\begin{array}{l}36 \\
33 \\
32 \\
30 \\
30\end{array}$ & $\begin{array}{l}44 \\
44 \\
44 \\
41 \\
44\end{array}$ & $\begin{array}{l}36 \\
33 \\
33 \\
32 \\
32\end{array}$ & $\begin{array}{l}28 \\
29 \\
32 \\
30 \\
30\end{array}$ & $\begin{array}{l}56 \\
53 \\
44 \\
39 \\
41\end{array}$ \\
\hline $\begin{array}{r}6 \\
7 \\
8 \\
9\end{array}$ & $\begin{array}{l}44 \\
42 \\
47 \\
47 \\
50\end{array}$ & $\begin{array}{l}29 \\
29 \\
29 \\
28 \\
26\end{array}$ & $\begin{array}{l}36 \\
30 \\
39 \\
44 \\
50\end{array}$ & \begin{tabular}{l|l}
33 & $b 4$ \\
28 & $b 3$ \\
26 & $b 2$ \\
26 & $b 2$ \\
26 & $b$
\end{tabular} & $\begin{array}{l}41 \\
30 \\
22 \\
28\end{array}$ & $\begin{array}{l}47 \\
47 \\
47 \\
47 \\
47\end{array}$ & $\begin{array}{l}36 \\
32 \\
39 \\
32 \\
30\end{array}$ & $\begin{array}{l}29 \\
29 \\
28 \\
28 \\
30\end{array}$ & $\begin{array}{l}44 \\
44 \\
44 \\
44 \\
44\end{array}$ & $\begin{array}{l}7.6 \\
2.3 \\
29 \\
30 \\
39\end{array}$ & $\begin{array}{l}29 \\
29 \\
33 \\
36 \\
30\end{array}$ & $\begin{array}{l}53 \\
41 \\
33 \\
29 \\
28\end{array}$ \\
\hline $\begin{array}{l}11 \\
12 \\
13 \\
15\end{array}$ & $\begin{array}{l}47 \\
47 \\
47 \\
47 \\
47\end{array}$ & $\begin{array}{l}25 \\
25 \\
39 \\
44 \\
36\end{array}$ & $\begin{array}{l}50 \\
44 \\
33 \\
29 \\
26\end{array}$ & \begin{tabular}{l|l}
25 & $b$ \\
24 & $b$ \\
23 & $b q$ \\
23 & $b q$ \\
23 & $b$
\end{tabular} & $\begin{array}{l}28 \\
25 \\
25 \\
26 \\
28\end{array}$ & $\begin{array}{l}47 \\
47 \\
44 \\
44 \\
44\end{array}$ & $\begin{array}{l}29 \\
28 \\
26 \\
26 \\
33\end{array}$ & $\begin{array}{l}39 \\
44 \\
44 \\
44 \\
30\end{array}$ & $\begin{array}{l}44 \\
44 \\
44 \\
44 \\
41\end{array}$ & $\begin{array}{l}50 \\
50 \\
50 \\
44 \\
50\end{array}$ & $\begin{array}{l}29 \\
26 \\
26 \\
25 \\
26\end{array}$ & $\begin{array}{l}28 \\
28 \\
32 \\
32 \\
32\end{array}$ \\
\hline $\begin{array}{l}16 \\
17 \\
18 \\
19 \\
20\end{array}$ & $\begin{array}{l}50 \\
47 \\
47 \\
47 \\
39\end{array}$ & $\begin{array}{l}32 \\
39 \\
50 \\
13.4 \\
0\end{array}$ & $\begin{array}{l}32 \\
29 \\
39 \\
36 \\
33\end{array}$ & \begin{tabular}{l|l}
22 & $b$ \\
23 & $b$ \\
27 & $b$ \\
25 & $b$ \\
24 & $b$
\end{tabular} & $\begin{array}{l}28 \\
33 \\
39 \\
44 \\
44\end{array}$ & $\begin{array}{l}44 \\
44 \\
39 \\
33 \\
32\end{array}$ & $\begin{array}{l}44 \\
44 \\
39 \\
24 \\
24\end{array}$ & $\begin{array}{l}29 \\
28 \\
36 \\
39 \\
44\end{array}$ & $\begin{array}{l}\mathbf{3 9} \\
\mathbf{3 6} \\
\mathbf{3 6} \\
33 \\
36\end{array}$ & $\begin{array}{l}53 \\
41 \\
36 \\
44 \\
56\end{array}$ & $\begin{array}{l}25 \\
25 \\
25 \\
25 \\
25\end{array}$ & $\begin{array}{l}29 \\
28 \\
30 \\
28 \\
26\end{array}$ \\
\hline 21 & $\begin{array}{l}36 \\
33 \\
42 \\
41 \\
32\end{array}$ & $\begin{array}{l}19.6 \\
39 \\
39 \\
29 \\
28\end{array}$ & $\begin{array}{l}44 \\
30 \\
26 \\
25 \\
23\end{array}$ & \begin{tabular}{l|l}
24 & $b$ \\
23 & $b$ \\
25 & $a$ \\
24 & \\
23 &
\end{tabular} & $\begin{array}{l}41 \\
44 \\
45 \\
41 \\
36\end{array}$ & $\begin{array}{l}30 \\
29 \\
29 \\
28 \\
37\end{array}$ & $\begin{array}{l}32 \\
44 \\
44 \\
44 \\
41\end{array}$ & $\begin{array}{l}44 \\
44 \\
44 \\
44 \\
44\end{array}$ & $\begin{array}{l}41 \\
41 \\
41 \\
41 \\
41\end{array}$ & $\begin{array}{l}41 \\
36 \\
33 \\
32 \\
30\end{array}$ & $\begin{array}{l}24 \\
24 \\
25 \\
25 \\
25\end{array}$ & $\begin{array}{l}26 \\
\mathbf{2 5} \\
25 \\
25 \\
25\end{array}$ \\
\hline $\begin{array}{l}26 \\
27 \\
28 \\
30 \\
31\end{array}$ & $\begin{array}{l}30 \\
44 \\
50 \\
50 \\
39 \\
36\end{array}$ & $\begin{array}{l}26 \\
29 \\
28 \\
26 \\
25 \\
24\end{array}$ & $\begin{array}{l}23 \\
23 \\
23 \\
36 \\
30\end{array}$ & $\begin{array}{l}20 \\
22 \\
22 \\
34 \\
41 \\
32\end{array}$ & $\begin{array}{l}36 \\
33 \\
33 \\
33 \\
33\end{array}$ & $\begin{array}{l}44 \\
39 \\
33 \\
41 \\
41 \\
33\end{array}$ & $\begin{array}{l}35 \\
32 \\
29 \\
41 \\
44 \\
39\end{array}$ & \begin{tabular}{l|}
44 \\
44 \\
44 \\
\hdashline- \\
.-
\end{tabular} & $\begin{array}{l}44 \\
39 \\
39 \\
41 \\
39 \\
39\end{array}$ & \begin{tabular}{|c|}
29 \\
29 \\
29 \\
28 \\
28 \\
\hdashline-9
\end{tabular} & $\begin{array}{l}25 \\
33 \\
56 \\
50 \\
50 \\
56\end{array}$ & $\begin{array}{r}24 \\
24 \\
24 \\
24 \\
23 \\
-2\end{array}$ \\
\hline \multirow{2}{*}{\multicolumn{4}{|c|}{ Month }} & \multicolumn{4}{|c|}{ Million gallons a day } & \multirow{2}{*}{\multicolumn{2}{|c|}{$\begin{array}{c}\text { Second- } \\
\text { feet } \\
\text { (mean) }\end{array}$}} & \multicolumn{3}{|c|}{ Total run-off } \\
\hline & & & & Maximum & \multicolumn{2}{|c|}{ Minimum } & Mean & & & $\begin{array}{l}\text { Million } \\
\text { gallons }\end{array}$ & \multicolumn{2}{|c|}{ Acre-feet } \\
\hline \multirow{2}{*}{\multicolumn{4}{|c|}{$\begin{array}{l}\text { July-ust } \\
\text { August } 30 \text { days) } \\
\text { September } \\
\text { Octoter } \\
\text { November } \\
\text { December } \\
\text { January } \\
\text { February } \\
\text { March } \\
\text { Aprik } \\
\text { May- } \\
\text { June }\end{array}$}} & $\begin{array}{l}50 \\
50 \\
50 \\
47 \\
-47 \\
44 \\
44 \\
44 \\
56 \\
56 \\
56\end{array}$ & & $\begin{array}{l}30 \\
0 \\
23 \\
20 \\
19.5 \\
28 \\
24 \\
28 \\
33 \\
2.3 \\
24 \\
23\end{array}$ & $\begin{array}{l}43.0 \\
31.6 \\
34.3 \\
27.3 \\
32.6 \\
39.1 \\
35.2 \\
36.9 \\
41.3 \\
35.4 \\
30.8 \\
31.8\end{array}$ & & $\begin{array}{l}66.5 \\
48.9 \\
53.1 \\
42.2 \\
50.4 \\
60.5 \\
54.5 \\
57.1 \\
63.9 \\
54.8 \\
47.7 \\
49.2\end{array}$ & $\begin{array}{r}1,330 \\
947 \\
1,030 \\
845 \\
978 \\
1,210 \\
1,090 \\
1,030 \\
1,280 \\
1,060 \\
956 \\
955\end{array}$ & \multicolumn{2}{|r|}{$\begin{array}{l}4,090 \\
2,910 \\
3,160 \\
2,600 \\
3,000 \\
3,720 \\
3,350 \\
3,170 \\
3,930 \\
3,260 \\
2,930 \\
2.930\end{array}$} \\
\hline & & & & 56 & & 0 & 35.0 & & 54.2 & 12.700 & & 39,000 \\
\hline
\end{tabular}




\section{KEKAHA DITCH BELOW TUNNEL NO, 12, FEAR WAMMEA, KAUAI}

Location.- Water-stage recorder 1 mile north of Waimea, just above diversion for Waimea domestic water supply.

Records available.-April, 1908, to November, 1914; July, 1916, to June, 1929.

Extremes.-Maximum discharge during year, 53 million gallons a day or 82 second-feet June 1 (gage height, 4.09 feet); no flow several days during August and April.

1908-1929: Maximum discharge, 70 million gallons a day or 108 secondfeet Dec. 24, 1927 (gage height, 5.17 feet); no flow occasionally when water was shut out of ditch.

REMARKs. - Records good except those where shifting-control method is used, which are fair. Intake on Waimea River 8 miles north of Waimea. Water used for irrigation of sugar cane near Kekaha. Regulated by head gates.

Discharge, in million gallons a day, 1928-29

\begin{tabular}{|c|c|c|c|c|c|c|c|c|c|c|c|c|}
\hline Day & July & Aug. & Sept. & Oct. & Nov. & Dec. & Jan. & Feb. & Mar. & Apr. & May & June \\
\hline $\begin{array}{l}1 \ldots \\
2 \ldots \\
3 \ldots \\
4 \ldots \\
5 \ldots\end{array}$ & $\begin{array}{l}32 \\
43 \\
34 \\
29 \\
43\end{array}$ & $\begin{array}{l}34 \\
39 \\
34 \\
30 \\
29\end{array}$ & $\begin{array}{l}19.2 \\
23 \\
36 \\
43 \\
43\end{array}$ & $\begin{array}{l}22 \\
35 \\
43 \\
30 \\
26\end{array}$ & $\begin{array}{l}24 \\
21 \\
19.2 \\
19.2 \\
34\end{array}$ & $\begin{array}{l}30 \\
29 \\
30 \\
29 \\
37\end{array}$ & $\begin{array}{l}23 \\
27 \\
32 \\
39 \\
37\end{array}$ & $\begin{array}{l}30 \\
27 \\
27 \\
25 \\
25\end{array}$ & $\begin{array}{l}36 \\
36 \\
36 \\
37 \\
37\end{array}$ & $\begin{array}{l}32 \\
30 \\
30 \\
29 \\
29\end{array}$ & $\begin{array}{l}24 \\
25 \\
29 \\
27 \\
26\end{array}$ & $\begin{array}{l}50 \\
48 \\
41 \\
36 \\
34\end{array}$ \\
\hline $\begin{array}{r}6 \\
7 \\
8 \\
9 \\
10 \ldots\end{array}$ & $\begin{array}{l}37 \\
34 \\
43 \\
41 \\
41\end{array}$ & $\begin{array}{l}27 \\
26 \\
27 \\
26 \\
24\end{array}$ & $\begin{array}{l}34 \\
27 \\
32 \\
37 \\
41\end{array}$ & $\begin{array}{l}29 \\
24 \\
22 \\
22 \\
22\end{array}$ & $\begin{array}{l}37 \\
27 \\
21 \\
23 \\
22\end{array}$ & $\begin{array}{l}41 \\
41 \\
41 \\
41 \\
41\end{array}$ & $\begin{array}{l}32 \\
29 \\
34 \\
29 \\
27\end{array}$ & $\begin{array}{l}24 \\
24 \\
23 \\
23 \\
23\end{array}$ & $\begin{array}{l}37 \\
37 \\
37 \\
37 \\
37\end{array}$ & $\begin{array}{l}\text { a } 8 \\
\text { a } 1.5 \\
a 26 \\
27 \\
30\end{array}$ & $\begin{array}{l}25 \\
24 \\
26 \\
32 \\
26\end{array}$ & $\begin{array}{l}43 \\
36 \\
29 \\
25 \\
24\end{array}$ \\
\hline $\begin{array}{l}11 \\
12 \\
13 \\
15\end{array}$ & $\begin{array}{l}39 \\
37 \\
37 \\
39 \\
37\end{array}$ & $\begin{array}{l}23 \\
23 \\
34 \\
37 \\
34\end{array}$ & $\begin{array}{l}41 \\
39 \\
30 \\
25 \\
22\end{array}$ & $\begin{array}{l}21 \\
20 \\
19.2 \\
19.2 \\
19.2\end{array}$ & $\begin{array}{l}22 \\
21 \\
21 \\
21 \\
22\end{array}$ & $\begin{array}{l}41 \\
41 \\
39 \\
39 \\
39\end{array}$ & $\begin{array}{l}25 \\
23 \\
22 \\
22 \\
26\end{array}$ & $\begin{array}{l}34 \\
39 \\
39 \\
37 \\
29\end{array}$ & $\begin{array}{l}37 \\
37 \\
37 \\
37 \\
36\end{array}$ & $\begin{array}{l}43 \\
43 \\
43 \\
41 \\
41\end{array}$ & $\begin{array}{l}23 \\
22 \\
22 \\
23 \\
23\end{array}$ & $\begin{array}{l}23 \\
23 \\
23 \\
25 \\
24\end{array}$ \\
\hline 18 & $\begin{array}{l}41 \\
41 \\
39 \\
37 \\
34\end{array}$ & $\begin{array}{l}30 \\
32 \\
43 \\
a 8 \\
a_{0} 0\end{array}$ & $\begin{array}{l}26 \\
24 \\
32 \\
32 \\
29\end{array}$ & $\begin{array}{l}19.2 \\
18.3 \\
21 \\
21 \\
20\end{array}$ & $\begin{array}{l}23 \\
25 \\
34 \\
39 \\
39\end{array}$ & $\begin{array}{l}37 \\
39 \\
34 \\
30 \\
29\end{array}$ & $\begin{array}{l}34 \\
34 \\
34 \\
19.2 \\
17.5\end{array}$ & $\begin{array}{l}26 \\
24 \\
30 \\
32 \\
34\end{array}$ & $\begin{array}{l}34 \\
32 \\
32 \\
30 \\
30\end{array}$ & $\begin{array}{l}43 \\
39 \\
34 \\
30 \\
43\end{array}$ & $\begin{array}{l}23 \\
23 \\
23 \\
22 \\
22\end{array}$ & $\begin{array}{l}23 \\
22 \\
24 \\
23 \\
22\end{array}$ \\
\hline 21 & $\begin{array}{l}30 \\
29 \\
32 \\
37 \\
29\end{array}$ & $\begin{array}{r}\text { a } 15 \\
34 \\
34 \\
27 \\
24\end{array}$ & $\begin{array}{l}37 \\
27 \\
23 \\
21 \\
20\end{array}$ & $\begin{array}{l}19.2 \\
19.2 \\
21 \\
21 \\
19.2\end{array}$ & $\begin{array}{l}37 \\
37 \\
39 \\
36 \\
32\end{array}$ & $\begin{array}{l}29 \\
27 \\
26 \\
26 \\
32\end{array}$ & $\begin{array}{l}22 \\
32 \\
36 \\
37 \\
34\end{array}$ & $\begin{array}{l}37 \\
36 \\
36 \\
36 \\
36\end{array}$ & $\begin{array}{l}36 \\
37 \\
37 \\
36 \\
37\end{array}$ & $\begin{array}{l}39 \\
32 \\
29 \\
27 \\
26\end{array}$ & $\begin{array}{l}22 \\
22 \\
24 \\
25 \\
25\end{array}$ & $\begin{array}{l}22 \\
21 \\
21 \\
20 \\
19.2\end{array}$ \\
\hline $\begin{array}{l}26 \\
27 \\
28 \\
30 \\
31\end{array}$ & $\begin{array}{l}27 \\
36 \\
41 \\
41 \\
34 \\
30\end{array}$ & $\begin{array}{l}22 \\
24 \\
23 \\
22 \\
22 \\
20\end{array}$ & $\begin{array}{l}19.2 \\
19.2 \\
18.3 \\
30 \\
26\end{array}$ & $\begin{array}{l}18.3 \\
18.3 \\
18.3 \\
27 \\
37 \\
29\end{array}$ & $\begin{array}{l}30 \\
30 \\
30 \\
29 \\
29 \\
-\end{array}$ & $\begin{array}{l}39 \\
36 \\
30 \\
36 \\
37 \\
30\end{array}$ & $\begin{array}{l}29 \\
27 \\
23 \\
34 \\
36 \\
32\end{array}$ & $\begin{array}{r}36 \\
36 \\
37 \\
-1 \\
\end{array}$ & $\begin{array}{l}37 \\
36 \\
34 \\
36 \\
36 \\
34\end{array}$ & $\begin{array}{l}26 \\
24 \\
24 \\
24 \\
23\end{array}$ & $\begin{array}{l}25 \\
30 \\
48 \\
46 \\
48 \\
50\end{array}$ & $\begin{array}{l}20 \\
20 \\
19.2 \\
19.2 \\
18.3 \\
\end{array}$ \\
\hline \multirow{2}{*}{\multicolumn{4}{|c|}{ Month }} & \multicolumn{4}{|c|}{ Million gallons a day } & \multirow{2}{*}{\multicolumn{2}{|c|}{$\begin{array}{l}\text { Second- } \\
\text { feet } \\
\text { (mean) }\end{array}$}} & \multicolumn{3}{|c|}{ Total run-off } \\
\hline & & & & Maximu & n Min & imum & Mean & & & $\begin{array}{l}\text { Million } \\
\text { gallons }\end{array}$ & Acr & e-feet \\
\hline \multicolumn{4}{|c|}{$\begin{array}{l}\text { July } \\
\text { August }(\mathbf{3 0} \text { days) } \\
\text { September.. } \\
\text { October } \\
\text { November } \\
\text { December } \\
\text { January } \\
\text { February } \\
\text { March } \\
\text { April } \\
\text { May } \\
\text { June }\end{array}$} & & 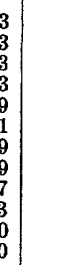 & $\begin{array}{l}27 \\
0 \\
18.3 \\
18.3 \\
19.2 \\
26 \\
17.5 \\
23 \\
30 \\
1.5 \\
22 \\
18.3\end{array}$ & $\begin{array}{l}36.3 \\
27.6 \\
29.2 \\
23.2 \\
28.1 \\
34.7 \\
29.3 \\
30.9 \\
35.6 \\
30.6 \\
27.6 \\
26.6\end{array}$ & & $\begin{array}{l}56.2 \\
42.7 \\
45,2 \\
35.9 \\
43.5 \\
53.7 \\
45.3 \\
47.8 \\
55.1 \\
47.3 \\
42.7 \\
41.2\end{array}$ & $\begin{array}{r}1,120 \\
827 \\
876 \\
721 \\
843 \\
1,080 \\
908 \\
\quad 865 \\
1,110 \\
916 \\
855 \\
798\end{array}$ & & $\begin{array}{l}3,450 \\
2,540 \\
2,690 \\
2,210 \\
2,590 \\
3,300 \\
2.790 \\
2.660 \\
3,390 \\
2,820 \\
2,630 \\
2,450\end{array}$ \\
\hline \multicolumn{4}{|c|}{ The year.................... } & & & 0 & 30.0 & & 46.4 & 10,900 & & 33,500 \\
\hline
\end{tabular}

- Estimated. 


\section{HANAPEPE RIVER AT KOULA, NEAR ELEEIR, KAUAT}

Location.-Water-stage recorder just below junction with Manuahi Stream, 500 feet below siphon at Koula, and 4 miles northeast of Eleele.

Drain AGE AREA. - 18.8 square miles.

Records available. - August, 1910, to January, 1921; and December, 1926, to June, 1929.

Extremes.-Maximum discharge during year, 969 million gallons a day or 1,500 second-feet Dec. 16 (gage height, 5.23 feet); minimum, 8.0 million gallons a day or 12.4 second-feet Jan. 9.

1910-1921, 1926-1929: Maximum discharge, at least 5,000 million gallons a day or 7,740 second-feet Dec. 18, 1916 (at old station above mouth of Manuahi Stream; gage height not known as station was destroyed by this flood); minimum, 7.1 million gallons a day or 11.0 second-feet Dec. 30, 31, 1913.

REMARKs.-Records fair for ordinary stages; poor for extremely high stages and estimated periods. Hanapepe ditch diverts water for irrigation from river 3 miles above station.

Discharge, in million gallons a day, 1928-29

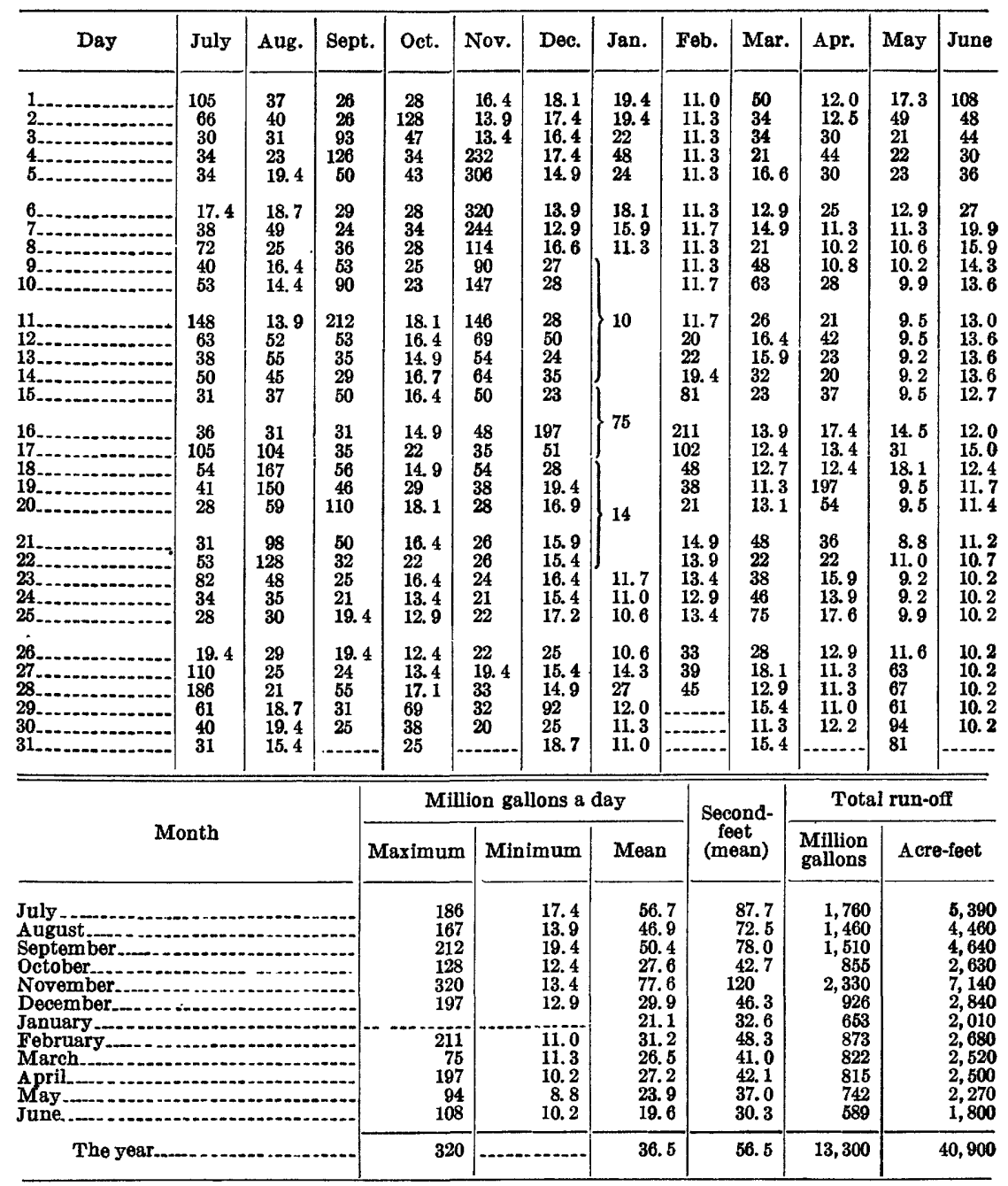




\section{HAN APEPE DITOE AT KOULA, FBAB RLEELE, KAUAI}

Location.-Water-stage recorder at first flume below siphon at Koula, 4 mile below intake and 4 miles north of Eleele.

Records avallable. -January, 1910, to June, 1921; March, 1927, to June, 1929.

Extremes.-Maximum discharge during year, 34 million gallons a day or 53 second-feet Apr. 19, May 19 (gage height, 3.05 feet); no flow several days when water was shut out of ditch.

1910-1921, 1927-1929: Maximum discharge, 36 million gallons a day or 56 second-feet Apr. 10, 1918 (gage height, 3.18 feet); ditch occasionally dry, owing to closing of head gates.

Remarks.-Records good. Diverts water for irrigation from Hanapepe River 3 miles above station. Regulated by head gates and spillways.

Discharge, in million gallons a day, 1928-29

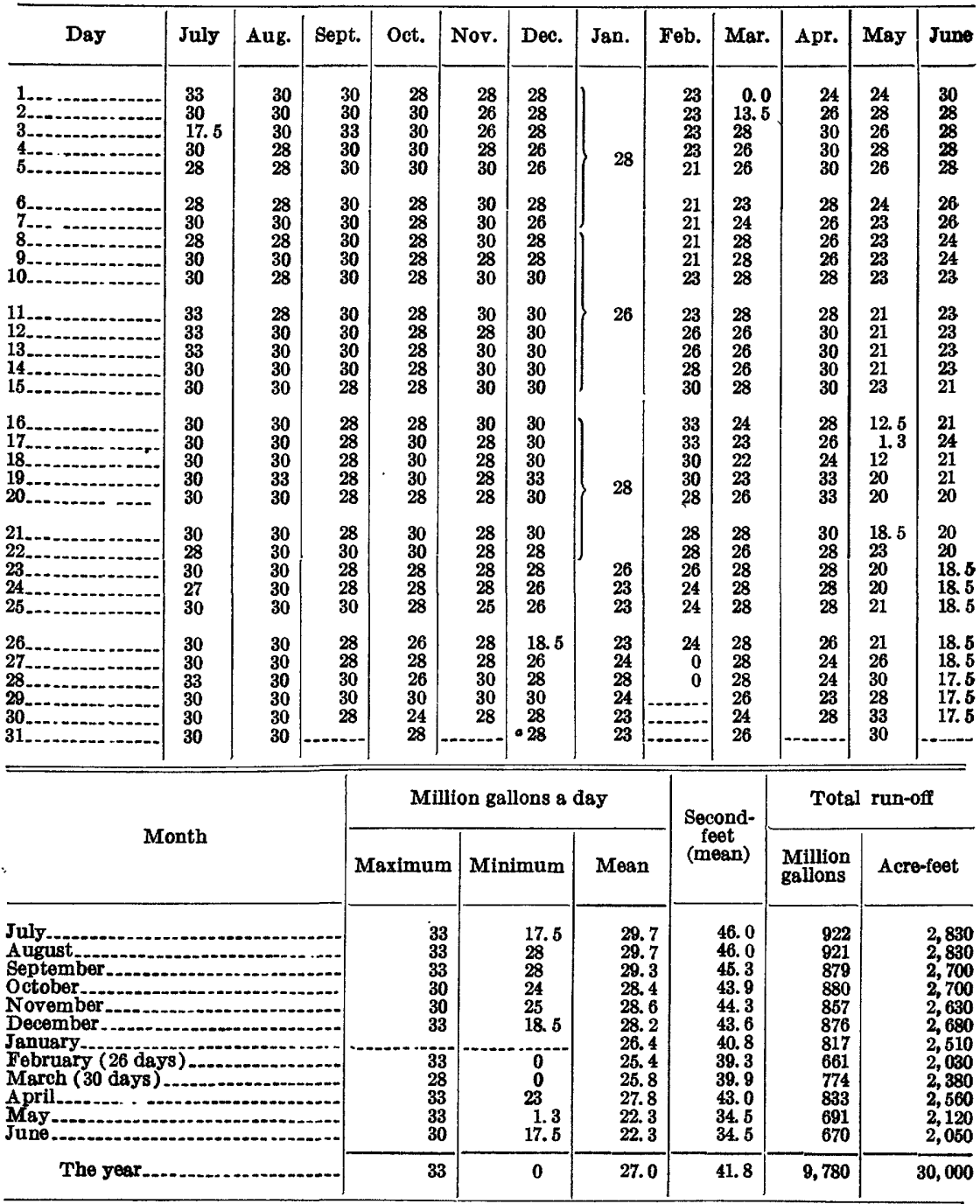

- Estimated. 


\section{SOUTH FORK OF WAIIUA RIVER NBAR LIHUE, KAUAI}

Locatron.-Water-stage recorder one-third mile above Wailua Falls and 5 miles northeast of Lihue. Prior to Nov. 18, 1918, one-third mile farther upstream.

DRAINAGE AREA.-22.4 square miles.

Records AvaILABLe.-December, 1911, to June, 1929.

EXTREMEs.-Maximum discharge during year, 12,600 million gallons a day or 19,500 second-feet May 30 (gage height, 8.44 feet); minimum, 2.0 million gallons a day or 3.1 second-feet Feb. 13 and 14 .

1911-1929: Maximum discharge, 28,000 million gallons a day or 44,900 second-feet Jan. 16, 1920 (gage height, 11.25 feet); minimum, 1.2 million gallons a day or 1.9 second-feet May 3,1926 .

REMARKs.- Records good except those for extremely high stages, which are poor. Lihue ditch and Hanamaulu ditch divert above station at elevations of 600 and 500 feet, respectively, for irrigation.

Discharge, in million gallons a day, 1928-29

\begin{tabular}{|c|c|c|c|c|c|c|c|c|c|c|c|c|c|}
\hline Day & July & Aug. & Sept. & Oct. & Nov. & Dec. & Jan. & Feb. & Mar. & Apr. & & Iay & June \\
\hline & $\begin{array}{l}43 \\
55 \\
13.7 \\
26 \\
11.8\end{array}$ & $\begin{array}{l}53 \\
64 \\
70 \\
31 \\
42\end{array}$ & $\begin{array}{r}4.7 \\
4.8 \\
75 \\
171 \\
58\end{array}$ & $\begin{array}{c}34 \\
300 \\
34 \\
13.4 \\
25\end{array}$ & $\begin{array}{r}23 \\
21 \\
31 \\
1,470 \\
725\end{array}$ & $\begin{array}{l}60 \\
55 \\
53 \\
48 \\
46\end{array}$ & $\begin{array}{l}23 \\
19.5 \\
5.9 \\
34 \\
32\end{array}$ & $\begin{array}{l}\text { 3. } 7 \\
\text { 3. } 6 \\
\text { 3. } 4 \\
\text { 3. } 2 \\
\text { 3. } 2\end{array}$ & $\begin{array}{l}6.0 \\
6.8 \\
28 \\
27 \\
26\end{array}$ & $\begin{array}{l}33 \\
28 \\
47 \\
36 \\
67\end{array}$ & & $\begin{array}{l}27 \\
48 \\
41 \\
36 \\
50\end{array}$ & $\begin{array}{l}190 \\
74 \\
49 \\
16.0 \\
28\end{array}$ \\
\hline $\begin{array}{r}6 .- \\
7-- \\
8 .- \\
9 .- \\
10 .-\end{array}$ & $\begin{array}{l}4.5 \\
4.5 \\
41 \\
22 \\
6.0\end{array}$ & $\begin{array}{l}27 \\
44 \\
35 \\
11.4 \\
4.2\end{array}$ & $\begin{array}{r}7.6 \\
4.5 \\
7.2 \\
42 \\
131\end{array}$ & $\begin{array}{c}22 \\
45 \\
6.0 \\
3.9 \\
3.6\end{array}$ & $\begin{array}{l}662 \\
481 \\
299 \\
217 \\
314\end{array}$ & $\begin{array}{l}45 \\
44 \\
45 \\
49 \\
52\end{array}$ & \begin{tabular}{r}
\multicolumn{1}{c}{24} \\
22 \\
15.6 \\
4.2 \\
3.7
\end{tabular} & $\begin{array}{l}\text { 3. } \\
\text { 3. } \\
3.0 \\
2.6 \\
2.6\end{array}$ & $\begin{array}{l}21 \\
13.3 \\
19.5 \\
26 \\
59\end{array}$ & $\begin{array}{l}62 \\
32 \\
18.2 \\
4.7 \\
29\end{array}$ & & $\begin{array}{r}15.2 \\
5.2 \\
3.6 \\
3.3 \\
3.3\end{array}$ & $\begin{array}{l}29 \\
8.7 \\
6.9 \\
6.3 \\
6.3\end{array}$ \\
\hline $\begin{array}{l}11 \\
12 \\
13 \\
14 \\
15\end{array}$ & $\begin{array}{c}108 \\
26 \\
8.6 \\
25 \\
26\end{array}$ & $\begin{array}{c}4.1 \\
15.7 \\
92 \\
23 \\
9.8\end{array}$ & $\begin{array}{c}403 \\
58 \\
18.1 \\
6.0 \\
55\end{array}$ & $\begin{array}{l}3.5 \\
3.4 \\
3.3 \\
3.2 \\
3.2\end{array}$ & $\begin{array}{l}294 \\
151 \\
125 \\
163 \\
134\end{array}$ & $\begin{array}{l}40 \\
21 \\
10.6 \\
45 \\
34\end{array}$ & $\begin{array}{l}4.0 \\
4.2 \\
4.2 \\
4.1 \\
4.1\end{array}$ & $\begin{array}{r}2.7 \\
2.3 \\
2.1 \\
3.5 \\
14.7\end{array}$ & \begin{tabular}{l|}
40 \\
28 \\
14.9 \\
20 \\
26
\end{tabular} & $\begin{array}{l}21 \\
9.5 \\
15.3 \\
61 \\
70\end{array}$ & & $\begin{array}{l}3.2 \\
3.1 \\
3.1 \\
3.1 \\
3.1\end{array}$ & $\begin{array}{l}6.1 \\
6.0 \\
5.4 \\
5.3 \\
5.2\end{array}$ \\
\hline $\begin{array}{l}16 \\
17 \\
18 \\
19 \\
20\end{array}$ & $\begin{array}{r}15.6 \\
74 . \\
16.4 \\
15.2 \\
8.0\end{array}$ & $\begin{array}{c}7.1 \\
75 \\
522 \\
386 \\
193\end{array}$ & $\begin{array}{c}38 \\
59 \\
41 \\
16.7 \\
351\end{array}$ & $\begin{array}{l}3.2 \\
3.4 \\
3.5 \\
3.4 \\
3.2\end{array}$ & $\begin{array}{r}152 \\
110 \\
129 \\
110 \\
81\end{array}$ & $\begin{array}{r}1,040 \\
149 \\
78 \\
55 \\
35\end{array}$ & $\begin{array}{c}4.0 \\
125 \\
165 \\
58 \\
52\end{array}$ & $\begin{array}{c}52 \\
70 \\
24 \\
4.2 \\
3.6\end{array}$ & $\begin{array}{r}16.7 \\
6.2 \\
16.7 \\
6.4 \\
4.3\end{array}$ & $\begin{array}{c}49 \\
26 \\
10.2 \\
240 \\
49\end{array}$ & & $\begin{array}{l}3.1 \\
3.0 \\
2.9 \\
3.1 \\
\text { 3. } 0\end{array}$ & $\begin{array}{l}5.2 \\
5.7 \\
5.2 \\
5.3 \\
5.3\end{array}$ \\
\hline $\begin{array}{l}21 \\
22 \\
23 \\
24 \\
25\end{array}$ & $\begin{array}{c}10.5 \\
52 \\
113 \\
42 \\
6.0\end{array}$ & $\begin{array}{r}215 \\
221 \\
48 \\
35 \\
28\end{array}$ & $\begin{array}{c}125 \\
54 \\
44 \\
22 \\
6.6\end{array}$ & $\begin{array}{l}3.3 \\
3.5 \\
3.4 \\
3.2 \\
3.1\end{array}$ & $\begin{array}{l}70 \\
78 \\
64 \\
53 \\
49\end{array}$ & $\begin{array}{l}17.5 \\
40 \\
33 \\
32 \\
38\end{array}$ & $\begin{array}{l}41 \\
37 \\
43 \\
33 \\
20\end{array}$ & $\begin{array}{r}2.9 \\
8.6 \\
11.2 \\
16.8 \\
12.0\end{array}$ & $\begin{array}{r}4.9 \\
4.1 \\
3.8 \\
96 \\
246\end{array}$ & $\begin{array}{l}100 \\
45 \\
23 \\
12.3 \\
15.8\end{array}$ & & $\begin{array}{l}3.0 \\
3.1 \\
3.1 \\
3.1 \\
3.0\end{array}$ & $\begin{array}{l}4.8 \\
4.6 \\
4.6 \\
4.6 \\
4.6\end{array}$ \\
\hline $\begin{array}{l}26 \\
27 \\
28 \\
29 \\
30 \\
31\end{array}$ & $\begin{array}{l}4.7 \\
144 \\
255 \\
100 \\
80 \\
63\end{array}$ & $\begin{array}{r}44 \\
7.3 \\
5.4 \\
5.2 \\
5.1 \\
4.9\end{array}$ & $\begin{array}{l}10.6 \\
10.6 \\
32 \\
11.6 \\
18.5 \\
-\end{array}$ & $\begin{array}{r}3.0 \\
3.2 \\
4.2 \\
44 \\
9.5 \\
11.1\end{array}$ & $\begin{array}{c}25 \\
6.6 \\
228 \\
141 \\
72 \\
-. .-\end{array}$ & $\begin{array}{c}36 \\
30 \\
15.0 \\
106 \\
37 \\
25\end{array}$ & $\begin{array}{l}5.8 \\
23 \\
36 \\
9.2 \\
4.6 \\
3.8\end{array}$ & $\begin{array}{l}37 \\
32 \\
20\end{array}$ & $\begin{array}{l}53 \\
34 \\
96 \\
82 \\
51 \\
48\end{array}$ & $\begin{array}{c}12.8 \\
6.2 \\
30 \\
19.0 \\
5.3\end{array}$ & & $\begin{array}{l}3.1 \\
5.2 \\
72 \\
12.8 \\
120 \\
308\end{array}$ & $\begin{array}{r}4.5 \\
4.2 \\
4.2 \\
4.1 \\
3.9 \\
---\end{array}$ \\
\hline \multirow{2}{*}{\multicolumn{4}{|c|}{ Month }} & \multicolumn{5}{|c|}{ Million gallons a day } & \multirow{2}{*}{$\begin{array}{c}\text { Second- } \\
\text { feet } \\
\text { (mean) }\end{array}$} & \multicolumn{4}{|c|}{ Total run-off } \\
\hline & & & & Ma & aximum & Minimum & $\mathrm{Me}$ & & & $\begin{array}{l}\text { Mil } \\
\text { gall }\end{array}$ & & Acr & a-feet \\
\hline \multicolumn{5}{|c|}{$\begin{array}{l}\text { July } \\
\text { August } \\
\text { September } \\
\text { October } \\
\text { November } \\
\text { December } \\
\text { January } \\
\text { February } \\
\text { March } \\
\text { April } \\
\text { May } \\
\text { June }\end{array}$} & $\begin{array}{r}255 \\
522 \\
403 \\
300 \\
1,470 \\
1,040 \\
165 \\
70 \\
246 \\
240 \\
1,120 \\
190\end{array}$ & $\begin{array}{r}4.5 \\
4.1 \\
4.5 \\
3.0 \\
6.6 \\
10.6 \\
3.7 \\
2.1 \\
3.8 \\
4.7 \\
2.9 \\
3.9\end{array}$ & & $\begin{array}{l}5.8 \\
5.1 \\
2.9 \\
9.7 \\
6 \\
7.9 \\
7.9 \\
2.5 \\
6.5 \\
9.2 \\
1.3 \\
17.1\end{array}$ & $\begin{array}{c}70.9 \\
116 \\
97.3 \\
30.5 \\
334 \\
121 \\
43.2 \\
19.3 \\
56.5 \\
60.7 \\
94.8 \\
26.5\end{array}$ & $\begin{array}{l}1, \\
2, \\
1, \\
6, \\
2, \\
1, \\
1, \\
1,\end{array}$ & & & $\begin{array}{r}4,360 \\
7,140 \\
\mathbf{5 , 7 9 0} \\
1,870 \\
19,900 \\
7,410 \\
2,650 \\
1,070 \\
3,470 \\
3,610 \\
5,830 \\
1,570\end{array}$ \\
\hline \multicolumn{4}{|c|}{ The year.....- } & & 1,470 & 2.1 & & 57.7 & 89.3 & & & & 64,700 \\
\hline
\end{tabular}


NORTH FORK OF WAIIUA RIVER AT ELEVATION 650 FEET, NEAR IIHO, KA UAI

LOCATION.-Water-stage recorder $1 \frac{112}{2}$ miles above intake of Kanaha ditch and $73 / 4$ miles northwest of Lihue.

Drain Age ARe A. - 6.6 square miles.

Records available. - August, 1910, to June, 1929.

EXTREMEs.-Maximum discharge during year, 2,450 million gallons a day or 3,790 second-feet May 30 (gage height, 7.25 feet); minimum, 19.8 million gallons a day or 30.6 second-feet June 25 .

1910-1929: Maximum discharge, 3,410 million gallons a day or 5,280 second-feet Dec. 24, 1927 (gage height, 8.46 feet); minimum, about 7.7 million gallons a day or 11.9 second-feet Apr. 27, 1926.

REMARKs. - Records good for ordinary stages; poor for high stages. Hanalei tunnel discharges water into stream, and North Wailua ditch diverts water from stream above station for irrigation.

Discharge, in million gallons a day, 1928-29

\begin{tabular}{|c|c|c|c|c|c|c|c|c|c|c|c|c|}
\hline Day & July & Aug. & Sept. & Oet. & Nov. & Dec. & Jan. & Feb. & Mar. & Apr. & May & June \\
\hline $\begin{array}{l}1 \\
2 \\
3 \\
4 \\
5\end{array} \ldots .$. & $\begin{array}{l}98 \\
49 \\
38 \\
48 \\
52\end{array}$ & $\begin{array}{l}61 \\
63 \\
54 \\
43 \\
40\end{array}$ & $\begin{array}{r}37 \\
37 \\
88 \\
115 \\
56\end{array}$ & $\begin{array}{r}50 \\
155 \\
71 \\
57 \\
63\end{array}$ & $\begin{array}{r}46 \\
40 \\
40 \\
417 \\
252\end{array}$ & $\begin{array}{l}46 \\
43 \\
40 \\
40 \\
58\end{array}$ & $\begin{array}{l}49 \\
49 \\
91 \\
76 \\
49\end{array}$ & $\begin{array}{l}24 \\
24 \\
23 \\
23 \\
23\end{array}$ & $\begin{array}{l}43 \\
42 \\
71 \\
43 \\
83\end{array}$ & $\begin{array}{l}46 \\
57 \\
85 \\
64 \\
70\end{array}$ & $\begin{array}{l}66 \\
87 \\
46 \\
54 \\
49\end{array}$ & $\begin{array}{r}112 \\
97 \\
67 \\
56 \\
63\end{array}$ \\
\hline $\begin{array}{r}6 \\
7 \\
8 \\
9 \\
9\end{array}$ & $\begin{array}{l}34 \\
49 \\
71 \\
49 \\
44\end{array}$ & $\begin{array}{l}37 \\
93 \\
43 \\
37 \\
34\end{array}$ & $\begin{array}{r}43 \\
49 \\
71 \\
72 \\
100\end{array}$ & $\begin{array}{l}49 \\
57 \\
50 \\
39 \\
36\end{array}$ & $\begin{array}{l}255 \\
178 \\
147 \\
132 \\
158\end{array}$ & $\begin{array}{l}53 \\
43 \\
46 \\
57 \\
49\end{array}$ & $\begin{array}{l}40 \\
37 \\
40 \\
29 \\
29\end{array}$ & $\begin{array}{l}22 \\
22 \\
22 \\
21 \\
24\end{array}$ & $\begin{array}{l}53 \\
68 \\
76 \\
80 \\
80\end{array}$ & $\begin{array}{l}56 \\
37 \\
34 \\
45 \\
94\end{array}$ & $\begin{array}{l}37 \\
34 \\
37 \\
32 \\
29\end{array}$ & $\begin{array}{l}52 \\
43 \\
40 \\
37 \\
34\end{array}$ \\
\hline 15 & $\begin{array}{l}78 \\
54 \\
43 \\
40 \\
\mathbf{3 7}\end{array}$ & $\begin{array}{l}34 \\
90 \\
60 \\
48 \\
43\end{array}$ & $\begin{array}{r}170 \\
67 \\
52 \\
46 \\
60\end{array}$ & $\begin{array}{l}\mathbf{3 6} \\
\mathbf{3 4} \\
\mathbf{3 2} \\
\mathbf{3 5} \\
\mathbf{3 4}\end{array}$ & $\begin{array}{r}127 \\
95 \\
90 \\
100 \\
104\end{array}$ & $\begin{array}{l}52 \\
59 \\
55 \\
59 \\
61\end{array}$ & $\begin{array}{l}29 \\
27 \\
27 \\
27 \\
39\end{array}$ & $\begin{array}{l}35 \\
40 \\
32 \\
54 \\
87\end{array}$ & $\begin{array}{l}56 \\
46 \\
46 \\
57 \\
43\end{array}$ & $\begin{array}{l}63 \\
73 \\
46 \\
.43 \\
.52\end{array}$ & $\begin{array}{l}29 \\
28 \\
28 \\
28 \\
30\end{array}$ & $\begin{array}{l}37 \\
37 \\
34 \\
37 \\
32\end{array}$ \\
\hline 16 & $\begin{array}{r}40 \\
106 \\
62 \\
62 \\
43\end{array}$ & $\begin{array}{r}37 \\
62 \\
228 \\
149 \\
120\end{array}$ & $\begin{array}{r}40 \\
73 \\
-\quad 80 \\
-\quad 69 \\
192\end{array}$ & $\begin{array}{l}37 \\
47 \\
34 \\
\mathbf{4 7} \\
\mathbf{3 4}\end{array}$ & $\begin{array}{r}90 \\
85 \\
105 \\
80 \\
67\end{array}$ & $\begin{array}{r}263 \\
72 \\
52 \\
43 \\
40\end{array}$ & $\begin{array}{l}43 \\
83 \\
72 \\
37 \\
30\end{array}$ & $\begin{array}{r}107 \\
85 \\
52 \\
68 \\
43\end{array}$ & $\begin{array}{l}34 \\
34 \\
32 \\
29 \\
34\end{array}$ & $\begin{array}{r}40 \\
34 \\
38 \\
166 \\
82\end{array}$ & $\begin{array}{l}27 \\
27 \\
29 \\
29 \\
28\end{array}$ & $\begin{array}{l}32 \\
32 \\
29 \\
34 \\
29\end{array}$ \\
\hline 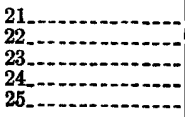 & $\begin{array}{l}43 \\
72 \\
87 \\
46 \\
49\end{array}$ & $\begin{array}{r}176 \\
131 \\
76 \\
59 \\
56\end{array}$ & $\begin{array}{l}94 \\
\mathbf{5 9} \\
\mathbf{4 6} \\
40 \\
55\end{array}$ & $\begin{array}{l}37 \\
40 \\
32 \\
32 \\
32\end{array}$ & $\begin{array}{l}64 \\
90 \\
63 \\
56 \\
52\end{array}$ & $\begin{array}{l}37 \\
34 \\
34 \\
34 \\
80\end{array}$ & $\begin{array}{l}36 \\
38 \\
43 \\
29 \\
27\end{array}$ & $\begin{array}{l}34 \\
42 \\
32 \\
29 \\
29\end{array}$ & $\begin{array}{r}73 \\
54 \\
61 \\
131 \\
156\end{array}$ & $\begin{array}{l}72 \\
52 \\
46 \\
43 \\
46\end{array}$ & $\begin{array}{l}28 \\
28 \\
28 \\
29 \\
34\end{array}$ & $\begin{array}{l}27 \\
25 \\
20 \\
20 \\
20\end{array}$ \\
\hline $\begin{array}{l}26 \\
27 \\
28 \\
30 \\
31\end{array}$ & $\begin{array}{r}43 \\
110 \\
136 \\
77 \\
52 \\
46\end{array}$ & $\begin{array}{l}49 \\
46 \\
37 \\
34 \\
34 \\
29\end{array}$ & $\begin{array}{l}46 \\
48 \\
57 \\
43 \\
37\end{array}$ & $\begin{array}{l}32 \\
38 \\
46 \\
78 \\
50 \\
48\end{array}$ & $\begin{array}{r}49 \\
46 \\
108 \\
59 \\
52 \\
-\end{array}$ & $\begin{array}{r}40 \\
40 \\
60 \\
128 \\
49 \\
46\end{array}$ & $\begin{array}{l}27 \\
54 \\
43 \\
27 \\
26 \\
25\end{array}$ & $\begin{array}{r}59 \\
34 \\
52 \\
\\
\\
\end{array}$ & $\begin{array}{l}67 \\
52 \\
60 \\
58 \\
43 \\
69\end{array}$ & $\begin{array}{l}\mathbf{3 7} \\
\mathbf{3 4} \\
\mathbf{3 4} \\
\mathbf{3 4} \\
\mathbf{4 2}\end{array}$ & $\begin{array}{r}32 \\
65 \\
63 \\
75 \\
342 \\
127\end{array}$ & $\begin{array}{l}21 \\
22 \\
24 \\
25 \\
25\end{array}$ \\
\hline
\end{tabular}

\begin{tabular}{|c|c|c|c|c|c|c|}
\hline \multirow[b]{2}{*}{ Month } & \multicolumn{3}{|c|}{ Million gallons a day } & \multirow{2}{*}{$\begin{array}{l}\text { Second- } \\
\text { feet } \\
\text { (mean) }\end{array}$} & \multicolumn{2}{|c|}{ Total run-off } \\
\hline & Maximum & Minimum & Mean & & $\begin{array}{l}\text { Million } \\
\text { gallons }\end{array}$ & Acre-feet \\
\hline $\begin{array}{l}\text { July } \\
\text { August } \\
\text { September } \\
\text { October. } \\
\text { November } \\
\text { December } \\
\text { January } \\
\text { February } \\
\text { March } \\
\text { April } \\
\text { May }\end{array}$ & $\begin{array}{r}136 \\
228 \\
192 \\
155 \\
417 \\
263 \\
91 \\
107 \\
156 \\
166 \\
342 \\
112\end{array}$ & $\begin{array}{l}34 \\
29 \\
37 \\
32 \\
40 \\
34 \\
25 \\
21 \\
29 \\
34 \\
27 \\
20\end{array}$ & $\begin{array}{r}59.9 \\
67.8 \\
68.1 \\
47.2 \\
108 \\
58.5 \\
41.2 \\
40.8 \\
60.5 \\
55.5 \\
51.8 \\
38.8\end{array}$ & $\begin{array}{r}92.7 \\
105 \\
105 \\
73.0 \\
167 \\
90.5 \\
63.7 \\
63.1 \\
93.6 \\
85.9 \\
80.1 \\
60.0\end{array}$ & $\begin{array}{l}1,860 \\
2,100 \\
2,040 \\
1,460 \\
3,250 \\
1,810 \\
1,280 \\
1,140 \\
1,870 \\
1,660 \\
1,600 \\
1,160\end{array}$ & $\begin{array}{l}5,700 \\
6,450 \\
6,270 \\
4,490 \\
9,940 \\
5,570 \\
3,920 \\
3,510 \\
5,760 \\
5,110 \\
4,930 \\
3,570\end{array}$ \\
\hline The year........... & 417 & 20 & 58.2 & 90.0 & 21,200 & 65,200 \\
\hline
\end{tabular}


KANAFA DTTOH NEAR IIHUE, RAUAI

LOCATION.-Water-stage recorder a quarter of a mile below Kauai Electric Co.'s power line and $6 \% 4$ miles northwest of Lihue.

Records av aILABLe.- August, 1910, to June, 1929.

EXTREMEs.- Maximum discharge during year, 41 million gallons a day or 63 second-feet May 30 (gage height, 3.07 feet); minimum, 0.6 million gallons a day or 0.9 second-foot Dec. 1 .

1910-1929: Maximum discharge recorded, 45 million gallons a day or 70 second-feet Dec. 24, 1927 (gage height, 3.22 feet); no fow occasionally when water was shut out of ditch.

REMARKs.-Records good. Intake $8 \frac{1}{2}$ miles above mouth of North Fork of Wailua River at elevation of about 600 feet. Water used for irrigation of sugar cane. Regulated by head gates and spillways.

Discharge, in million gallons a day, 1928-29

\begin{tabular}{|c|c|c|c|c|c|c|c|c|c|c|c|c|}
\hline Day & July & Aug. & Sept. & \begin{tabular}{l|l} 
Oct. & N
\end{tabular} & Nor. & ec. & Jan. & Feb. & Mar. & Apr. & May & June \\
\hline & $\begin{array}{l}8.9 \\
8.9 \\
8.9 \\
8.9\end{array}$ & $\begin{array}{l}8.9 \\
8.9 \\
8.9 \\
8.9 \\
8.9\end{array}$ & 8. & $\begin{array}{l}8.9 \\
8.9 \\
8.9 \\
8.3 \\
8.3\end{array}$ & $\begin{array}{l}8.3 \\
8.3 \\
8.3 \\
6.3 \\
2.0\end{array}$ & $\begin{array}{r}0.7 \\
.8 \\
.8 \\
.8 \\
1.2\end{array}$ & $\begin{array}{r}0.9 \\
.9 \\
.9 \\
.8 \\
.8\end{array}$ & $\begin{array}{l}7.0 \\
5.0 \\
4.1 \\
4.1 \\
4.3\end{array}$ & $\begin{array}{l}7.3 \\
7.3 \\
7.1 \\
7.0 \\
7.3\end{array}$ & $\begin{array}{l}7 \\
9 \\
0 \\
8 \\
8\end{array}$ & $\begin{array}{l}.1 \\
2 \\
6 \\
9 \\
.7\end{array}$ & 4. \\
\hline $\begin{array}{l}6 \\
8 \\
9\end{array}$ & $\begin{array}{l}8.9 \\
8.9 \\
8.9 \\
8.9 \\
8.9\end{array}$ & $\begin{array}{l}8.9 \\
8.9 \\
8.3 \\
8.9 \\
8.9\end{array}$ & $\begin{array}{l}8.9 \\
8.9 \\
8.9 \\
8.9 \\
8.9\end{array}$ & $\begin{array}{l}8.3 \\
8.9 \\
8.3 \\
8.3 \\
8.3\end{array}$ & $\begin{array}{l}2.5 \\
2.0 \\
1.8 \\
1.6 \\
1.6\end{array}$ & $\begin{array}{l}1.1 \\
1.1 \\
1.1 \\
1.1 \\
1.1\end{array}$ & $\begin{array}{r}.8 \\
.8 \\
.8 \\
3.3 \\
6.1\end{array}$ & $\begin{array}{l}4.4 \\
4.4 \\
4.4 \\
5.4 \\
7.1\end{array}$ & $\begin{array}{l}7.5 \\
7.8 \\
7.3 \\
7.2 \\
7.2\end{array}$ & $\begin{array}{l}6.9 \\
6.7 \\
6.8 \\
6.9 \\
7.0\end{array}$ & $\begin{array}{l}7.6 \\
7.8 \\
8.1 \\
8.1 \\
8.1\end{array}$ & $\begin{array}{l}7 . \\
7 .\end{array}$ \\
\hline (n) & $\begin{array}{l}8.9 \\
8.9 \\
8.9 \\
8.9 \\
8.9\end{array}$ & $\begin{array}{l}8.9 \\
8.9 \\
8.3 \\
8.3 \\
8.9\end{array}$ & $\begin{array}{l}9.5 \\
8.9 \\
8.9 \\
8.9 \\
8.9\end{array}$ & $\begin{array}{l}8.3 \\
8.3 \\
8.3 \\
8.3 \\
8.3\end{array}$ & $\begin{array}{l}1.4 \\
1.4 \\
1.2 \\
1.2 \\
1.2\end{array}$ & $\begin{array}{r}1.0 \\
1.0 \\
1.0 \\
.9 \\
.9\end{array}$ & $\begin{array}{l}7.8 \\
7.8 \\
7.9 \\
7.8 \\
7.9\end{array}$ & $\begin{array}{l}6.8 \\
6.8 \\
6.6 \\
7.1 \\
6.9\end{array}$ & $\begin{array}{l}7.1 \\
7.2 \\
7.3 \\
7.1 \\
7.0\end{array}$ & $\begin{array}{l}6.8 \\
6.7 \\
6.6 \\
6.4 \\
6.8\end{array}$ & $\begin{array}{l}7.8 \\
7.6\end{array}$ & 7. \\
\hline - & $\begin{array}{l}8.9 \\
8.9 \\
8.9 \\
8.9 \\
8.9\end{array}$ & $\begin{array}{l}8.3 \\
8.3 \\
8.3 \\
8.2 \\
8.3\end{array}$ & $\begin{array}{l}8.9 \\
8.9 \\
8.9 \\
8.9 \\
8.9\end{array}$ & \begin{tabular}{l|}
8.3 \\
8.3 \\
8.3 \\
8.3 \\
8.3
\end{tabular} & $\begin{array}{l}1.2 \\
1.1 \\
1.0 \\
1.0 \\
1.2\end{array}$ & $\begin{array}{r}1.0 \\
.8 \\
.8 \\
.9 \\
1.0\end{array}$ & $\begin{array}{l}5.6 \\
4.5 \\
4.5 \\
4.3 \\
4.2\end{array}$ & $\begin{array}{l}7.1 \\
7.0 \\
6.8 \\
7.1 \\
6.8\end{array}$ & $\begin{array}{l}7.22 \\
7.2 \\
7.2 \\
7.2 \\
7.2\end{array}$ & $\begin{array}{l}6.6 \\
6.3 \\
6.4 \\
7.1 \\
6.9\end{array}$ & $\begin{array}{l}7.3 \\
7.5 \\
7.6 \\
7.6 \\
7.5\end{array}$ & $\begin{array}{l}8 . \\
7 . \\
7 . \\
7 .\end{array}$ \\
\hline $\mid$ & $\begin{array}{l}8.9 \\
8.9 \\
8.9 \\
8.9 \\
8.9\end{array}$ & $\begin{array}{l}8.3 \\
8.2 \\
8.3 \\
8.3 \\
8.3\end{array}$ & $\begin{array}{l}8.9 \\
8.9 \\
8.9 \\
8.9 \\
8.9\end{array}$ & $\begin{array}{l}8.3 \\
8.9 \\
8.3 \\
8.3 \\
8.3\end{array}$ & $\begin{array}{l}.8 \\
1.0 \\
1.2 \\
1.2 \\
1.2\end{array}$ & $\begin{array}{r}1.0 \\
.8 \\
.8 \\
.8 \\
.7\end{array}$ & $\begin{array}{l}4.2 \\
4.5 \\
4.6 \\
4.4 \\
4.3\end{array}$ & $\begin{array}{l}7.1 \\
7.5 \\
7.1 \\
7.2 \\
7.5\end{array}$ & $\begin{array}{l}7.2 \\
7.5 \\
7.5 \\
7.5 \\
7.2\end{array}$ & $\begin{array}{l}6.7 \\
6.6 \\
6.8 \\
7.3 \\
7.3\end{array}$ & $\begin{array}{r}7.6 \\
11.9 \\
15.0 \\
12.2 \\
7.7\end{array}$ & 7. \\
\hline - & $\begin{array}{l}8.9 \\
8.9 \\
8.9 \\
8.9 \\
8.9 \\
8.9\end{array}$ & $\begin{array}{l}8.3 \\
8.3 \\
8.3 \\
8.3 \\
8.3 \\
8.3\end{array}$ & $\begin{array}{l}8.9 \\
8.9 \\
8.3 \\
8.9 \\
8.9\end{array}$ & $\begin{array}{l}8.9 \\
8.9 \\
8.2 \\
7.9 \\
8.3 \\
8.3\end{array}$ & $\begin{array}{r}1.0 \\
1.1 \\
1.2 \\
.8 \\
.7\end{array}$ & $\begin{array}{l}.7 \\
.7 \\
.7 \\
.8 \\
.8 \\
.8\end{array}$ & $\begin{array}{l}4.2 \\
4.4 \\
5.0 \\
6.7 \\
6.9 \\
7.0\end{array}$ & $\begin{array}{r}7.5 \\
7.1 \\
7.3 \\
\hdashline . . . \\
\hdashline \\
\hdashline\end{array}$ & $\begin{array}{l}7.0 \\
7.1 \\
7.0 \\
6.9 \\
6.9 \\
7.1\end{array}$ & $\begin{array}{l}7.2 \\
7.2 \\
7.2 \\
7.3 \\
7.5\end{array}$ & $\begin{array}{l}7.6 \\
7.6 \\
7.5 \\
7.8 \\
9.8 \\
6.1\end{array}$ & $\begin{array}{l}6 . \\
6 . \\
6 . \\
5 . \\
5 .\end{array}$ \\
\hline \multirow{2}{*}{\multicolumn{4}{|c|}{ Month }} & \multicolumn{4}{|c|}{ Million gallons a day } & \multirow{2}{*}{\multicolumn{2}{|c|}{$\begin{array}{c}\text { Second- } \\
\text { feet } \\
\text { (mean) }\end{array}$}} & \multicolumn{3}{|c|}{ Total run-off } \\
\hline & & & & Iaximum & Min & num & Mean & & & $\begin{array}{l}\text { Mullion } \\
\text { gallons }\end{array}$ & Acre & $\theta$-feet \\
\hline \multirow{2}{*}{\multicolumn{4}{|c|}{$\begin{array}{l}\text { July } \\
\text { August } \\
\text { September } \\
\text { October } \\
\text { November. } \\
\text { December } \\
\text { January } \\
\text { February } \\
\text { March } \\
\text { A pril } \\
\text { May } \\
\text { June }\end{array}$}} & $\begin{array}{r}8.9 \\
8.9 \\
9.5 \\
8.9 \\
8.3 \\
1.2 \\
7.9 \\
7.5 \\
7.8 \\
7.5 \\
15.0 \\
8.3\end{array}$ & & $\begin{array}{r}8.9 \\
8.2 \\
8.3 \\
7.9 \\
.7 \\
.7 \\
4.8 \\
4.1 \\
6.9 \\
6.3 \\
6.1 \\
4.0\end{array}$ & $\begin{array}{l}8 . \\
8 . \\
8 . \\
8 . \\
2 . \\
4 . \\
6 . \\
7.2\end{array}$ & & $\begin{array}{l}13.8 \\
13.2 \\
13.7 \\
13.0 \\
3.34 \\
1.38 \\
6.71 \\
9.81 \\
1.2 \\
10.6 \\
12.7 \\
10.3\end{array}$ & $\begin{array}{c}276 \\
264 \\
266 \\
260 \\
64.8 \\
27.7 \\
135 \\
178 \\
224 \\
206 \\
254 \\
200\end{array}$ & & $\begin{array}{l}847 \\
812 \\
816 \\
798 \\
198 \\
85 \\
413 \\
545 \\
686 \\
632 \\
780 \\
614\end{array}$ \\
\hline & & & & $\overline{15.0}$ & & .7 & 6.45 & & 9.98 & 2,360 & & 23 \\
\hline
\end{tabular}




\section{BAST BRANCH OF TORTH FORK OF WAIIUA RIVER NEAR IHUE, KAUAI}

Location.- Water-stage recorder 1,200 feet above confluence with North Fork and $7 \frac{1}{2}$ miles northwest of Lihue.

DraIN AGE AREA.-6.2 square miles.

Records available. - July, 1912, to June, 1929.

Extremes.- Maximum discharge during year, 3,320 million gallons a day or 5,140 second-feet May 30 (gage height, 10.48 feet); minimum, 11.5 million gallons a day or 17.8 second-feet June 30 .

1912-1929: Maximum discharge, 3,340 million gallons a day or 5,170 secondfeet Dec. 24, 1927 (gage height, 10.57 feet); minimum, 4.4 million gallons a day or 6.8 second-feet July $8,13,1926$.

REMARKs.- Records good for ordinary stages and fair for high and low stages. No diversions above station.

Discharge, in million gallons a day, 1928-29

\begin{tabular}{|c|c|c|c|c|c|c|c|c|c|c|c|c|}
\hline Day & July & Aug. & Sept. & Oct. & Nov. & Dec. & Jan. & Feb. & Mar. & Apr. & May & June \\
\hline $\begin{array}{l}1 \\
2 \\
3 \\
3 \\
5\end{array} \ldots$ & $\begin{array}{l}19.1 \\
16.5 \\
15.5 \\
20 \\
19.2\end{array}$ & $\begin{array}{l}26 \\
27 \\
24 \\
22 \\
21\end{array}$ & $\begin{array}{l}19.2 \\
19.2 \\
31 \\
42 \\
26\end{array}$ & \begin{tabular}{l|l}
16.5 & \\
37 & \\
19.2 & \\
17.5 & 4 \\
19.2 & 20
\end{tabular} & $\begin{array}{l}15.5 \\
14.0 \\
14.0 \\
473 \\
206\end{array}$ & 24 & $\begin{array}{l}21 \\
21 \\
35 \\
39 \\
24\end{array}$ & $\begin{array}{l}13.2 \\
13.0 \\
12.8 \\
12.8 \\
12.8\end{array}$ & $\begin{array}{l}19.2 \\
17.5 \\
29 \\
22 \\
41\end{array}$ & $\begin{array}{l}22 \\
24 \\
26 \\
33 \\
26\end{array}$ & $\begin{array}{l}19.2 \\
23 \\
19.2 \\
19.2 \\
17.5\end{array}$ & $\begin{array}{l}64 \\
40 \\
35 \\
28 \\
30\end{array}$ \\
\hline 6. & $\begin{array}{l}16.2 \\
20 \\
33 \\
24 \\
22\end{array}$ & $\begin{array}{l}19.2 \\
25 \\
19.2 \\
17.5 \\
17.5\end{array}$ & $\begin{array}{l}21 \\
21 \\
24 \\
26 \\
26\end{array}$ & \begin{tabular}{l|l}
17.2 & 18 \\
16.8 & 1. \\
16.8 & \\
16.2 & \\
15.8 &
\end{tabular} & $\begin{array}{r}181 \\
117 \\
82 \\
67 \\
90\end{array}$ & $\begin{array}{l}21 \\
19.2 \\
19.2 \\
24 \\
21\end{array}$ & $\begin{array}{l}21 \\
19.2 \\
19.2 \\
17.5 \\
17.5\end{array}$ & $\begin{array}{l}12.5 \\
12.5 \\
12.3 \\
12.1 \\
15.3\end{array}$ & $\begin{array}{l}28 \\
32 \\
37 \\
37 \\
44\end{array}$ & $\begin{array}{l}22 \\
21 \\
21 \\
23 \\
51\end{array}$ & $\begin{array}{l}16.8 \\
16.5 \\
16.5 \\
15.8 \\
15.5\end{array}$ & $\begin{array}{l}26 \\
24 \\
22 \\
21 \\
21\end{array}$ \\
\hline $\begin{array}{l}11 \ldots \\
12 \ldots . . \\
13 \ldots \ldots \\
14 \ldots \ldots \\
16 \ldots . . .\end{array}$ & $\begin{array}{l}36 \\
24 \\
21 \\
21 \\
19.2\end{array}$ & $\begin{array}{l}17.5 \\
26 \\
24 \\
25 \\
21\end{array}$ & $\begin{array}{l}71 \\
26 \\
22 \\
21 \\
24\end{array}$ & $\begin{array}{l}15.6 \\
14.8 \\
15.0 \\
14.8 \\
16.2\end{array}$ & $\begin{array}{l}76 \\
42 \\
37 \\
37 \\
40\end{array}$ & $\begin{array}{r}21 \\
24 \\
25 \\
24 \\
21\end{array}$ & $\begin{array}{l}17.2 \\
16.8 \\
15.8 \\
15.5 \\
17.0\end{array}$ & $\begin{array}{l}16.0 \\
23 \\
14.2 \\
45 \\
49\end{array}$ & $\begin{array}{l}33 \\
28 \\
28 \\
28 \\
22\end{array}$ & $\begin{array}{l}35 \\
38 \\
28 \\
26 \\
30\end{array}$ & $\begin{array}{l}15.0 \\
14.5 \\
14.5 \\
14.8 \\
15.0\end{array}$ & $\begin{array}{l}19.2 \\
21 \\
19.2 \\
19.2 \\
17.5\end{array}$ \\
\hline 16 & $\begin{array}{l}18.3 \\
58 \\
28 \\
28 \\
22\end{array}$ & $\begin{array}{c}19.2 \\
24 \\
116 \\
64 \\
93\end{array}$ & $\begin{array}{l}21 \\
22 \\
21 \\
21 \\
92\end{array}$ & $\begin{array}{l}15.2 \\
16.5 \\
16.0 \\
15.8 \\
15.0\end{array}$ & $\begin{array}{l}38 \\
33 \\
42 \\
33 \\
28\end{array}$ & $\begin{array}{l}98 \\
30 \\
24 \\
22 \\
21\end{array}$ & $\begin{array}{l}21 \\
35 \\
39 \\
22 \\
19.2\end{array}$ & $\begin{array}{l}29 \\
39 \\
26 \\
36 \\
22\end{array}$ & $\begin{array}{l}21 \\
19.2 \\
19.2 \\
17.5 \\
21 .\end{array}$ & $\begin{array}{l}24 \\
22 \\
22 \\
67 \\
30\end{array}$ & $\begin{array}{l}14.5 \\
14.0 \\
13.8 \\
13.8 \\
13.8\end{array}$ & $\begin{array}{l}17.0 \\
16.8 \\
16.2 \\
16.8 \\
16.0\end{array}$ \\
\hline 21 & $\begin{array}{l}22 \\
30 \\
42 \\
24 \\
24\end{array}$ & $\begin{array}{l}69 \\
48 \\
33 \\
28 \\
26\end{array}$ & $\begin{array}{l}30 \\
24 \\
22 \\
21 \\
21\end{array}$ & $\begin{array}{l}14.5 \\
14.8 \\
14.2 \\
13.8 \\
13.2\end{array}$ & $\begin{array}{l}24 \\
30 \\
24 \\
22 \\
21\end{array}$ & $\begin{array}{l}19.2 \\
19.2 \\
17.5 \\
17.2 \\
32\end{array}$ & $\begin{array}{l}19.0 \\
21 \\
21 \\
17.0 \\
15.8\end{array}$ & $\begin{array}{l}19.2 \\
21 \\
19.2 \\
17.0 \\
19.6\end{array}$ & $\begin{array}{l}28 \\
21 \\
21 \\
74 \\
72\end{array}$ & $\begin{array}{l}36 \\
28 \\
26 \\
24 \\
24\end{array}$ & $\begin{array}{l}13.2 \\
14.0 \\
13.8 \\
13.2 \\
13.2\end{array}$ & $\begin{array}{l}15.2 \\
14.5 \\
14.2 \\
13.8 \\
13.6\end{array}$ \\
\hline $\begin{array}{l}26 \\
27 \\
28 \\
31\end{array}$ & $\begin{array}{l}21 \\
53 \\
37 \\
26 \\
24 \\
22\end{array}$ & $\begin{array}{l}24 \\
24 \\
21 \\
21 \\
19.2 \\
19.2\end{array}$ & \begin{tabular}{l}
21 \\
19.2 \\
22 \\
19.2 \\
17.5 \\
\hdashline
\end{tabular} & $\left.\begin{array}{l|l}13.0 & 2 \\
14.0 \\
14.0 \\
24 \\
19.0 \\
16.8\end{array}\right\}$ & $\begin{array}{l}24 \\
40\end{array}$ & $\begin{array}{l}19.2 \\
17.2 \\
16.8 \\
113 \\
24 \\
21\end{array}$ & $\begin{array}{l}15.2 \\
15.0 \\
16.8 \\
14.5 \\
13.8 \\
13.5\end{array}$ & \begin{tabular}{c|}
38 \\
22 \\
21 \\
\hdashline \\
\hdashline \\
\hdashline
\end{tabular} & $\begin{array}{l}35 \\
30 \\
34 \\
37 \\
26 \\
24\end{array}$ & \begin{tabular}{|l|}
21 \\
21 \\
19.2 \\
19.2 \\
19.2 \\
\hdashline$\cdots . .-$
\end{tabular} & $\begin{array}{l}14.0 \\
21 \\
43 \\
17.5 \\
396 \\
160\end{array}$ & \begin{tabular}{r}
13.0 \\
12.8 \\
12.3 \\
12.1 \\
11.0 \\
\hdashline \\
\end{tabular} \\
\hline \multirow{2}{*}{\multicolumn{4}{|c|}{ Month }} & \multicolumn{4}{|c|}{ Million gallons a day } & \multirow{2}{*}{\multicolumn{2}{|c|}{$\begin{array}{c}\text { Second- } \\
\text { feet } \\
\text { (mean) }\end{array}$}} & \multicolumn{3}{|c|}{ Total run-off } \\
\hline & & & & Aaximum & Min. & num & Mean & & & $\begin{array}{l}\text { Million } \\
\text { gallons }\end{array}$ & Acr & e-feet \\
\hline $\begin{array}{l}\text { July } \\
\text { August } \\
\text { September } \\
\text { October- } \\
\text { November } \\
\text { December } \\
\text { January } \\
\text { February } \\
\text { March } \\
\text { April } \\
\text { May } \\
\text { June. }\end{array}$ & $=$ & 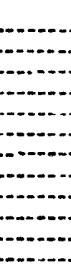 & & \begin{tabular}{r|}
58 \\
116 \\
92 \\
37 \\
473 \\
113 \\
39 \\
49 \\
74 \\
67 \\
396 \\
64
\end{tabular} & & $\begin{array}{l}15.5 \\
17.5 \\
17.5 \\
13.0 \\
14.0 \\
16.8 \\
13.5 \\
12.1 \\
17.5 \\
19.2 \\
13.2 \\
11.9\end{array}$ & $\begin{array}{l}26.0 \\
31.6 \\
27 . \\
16 . \\
65 . \\
27 . \\
20 . \\
21.6 \\
30 . \\
27 . \\
33 . \\
20 .\end{array}$ & & $\begin{array}{l}40.2 \\
48.9 \\
41.9 \\
25.8 \\
01 . \\
42.4 \\
31.7 \\
33.4 \\
47.2 \\
42.7 \\
52.0 \\
32.2\end{array}$ & $\begin{array}{r}806 \\
980 \\
813 \\
517 \\
1,970 \\
851 \\
636 \\
606 \\
946 \\
829 \\
1.040 \\
623\end{array}$ & & $\begin{array}{l}2,470 \\
3,010 \\
2,500 \\
1.590 \\
6 ; 040 \\
2,610 \\
1.950 \\
1.860 \\
2,900 \\
2.540 \\
3.200 \\
1,910\end{array}$ \\
\hline The year. & 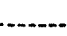 & 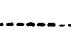 & & 473 & & 11.9 & 29.1 & & 45.0 & 10,600 & & 32,600 \\
\hline
\end{tabular}




\section{KAPAHI DITCH NEAR KEAIIA, KAUAI}

Location.-Water-stage recorder 500 feet below intake and 4.5 miles west of Kealia.

Records Available.-April, 1909, to May, 1914; May, 1915, to June, 1929.

EXTREMEs.-Maximum discharge during year, 123 million gallons a day or 190 second-feet Aug. 18 (gage height, 2.07 feet); no flow Sept. 3, when water was shut out of ditch.

1909-1914, 1915-1929: Maximum discharge, 233 million gallons a day or 361 second-feet Mar. 31, 1923 (gage height, about 3.15 feet); no flow occasionally when water is shut out of ditch.

REMARKs. - Records good except those for extremely low stages, which are poor. Diverts water from Kapaa River at elevation of about 400 feet. Water used for irrigation. Regulated by head gates.

Discharge, in million gallons a day, 1928-29

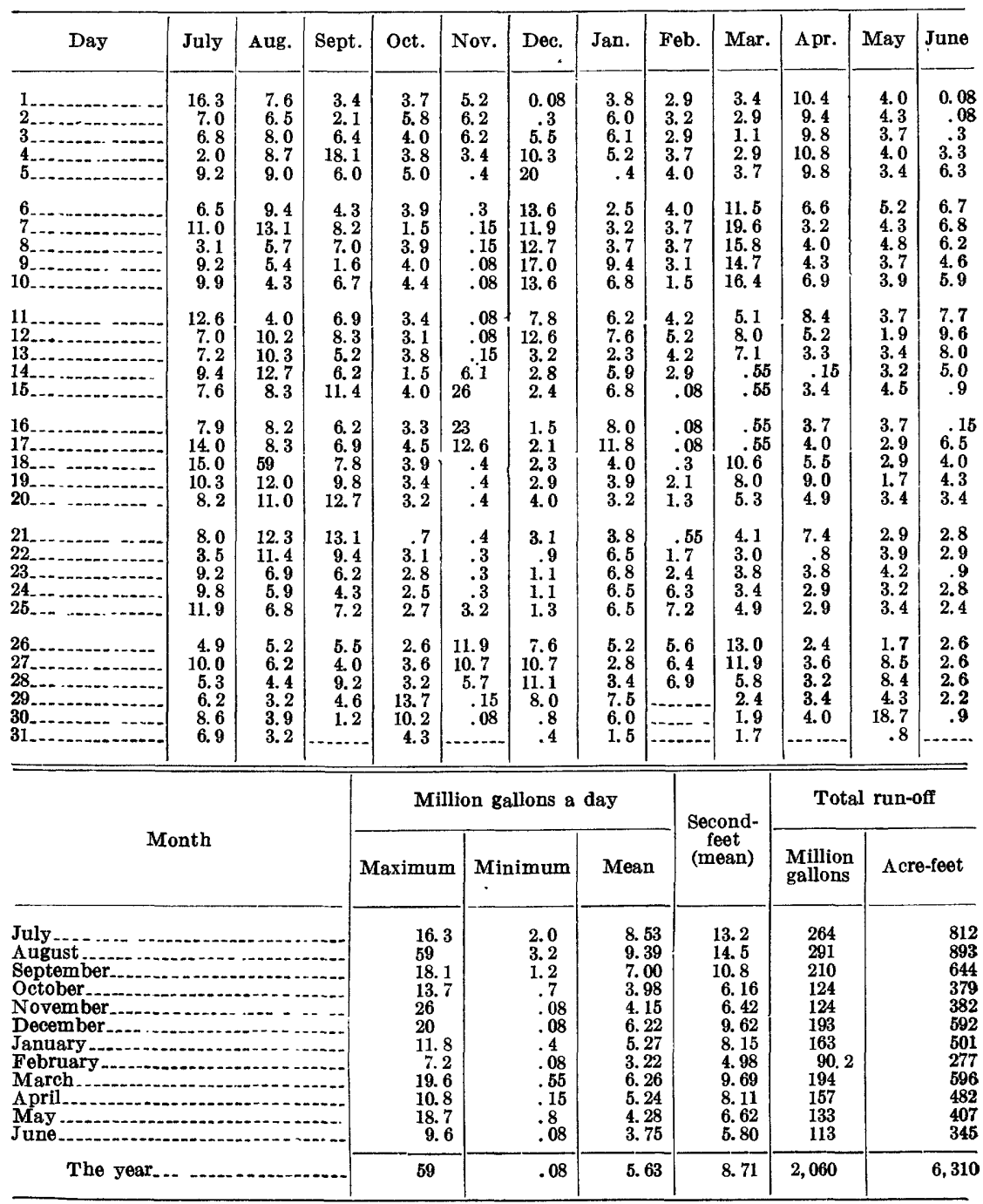




\section{ANAHOLA RIVER NEAR KEALIA, KAOAI}

Location.- Water-stage recorder a quarter of a mile above dam at Kiokala and $41 / 2$ miles northwest of Kealia.

Drainage AREA. $-\mathbf{5 . 5}$ square miles.

Records available.-August to November, 1910; December, 1912, to June, 1929.

Extrames.-Maximum discharge during year, 875 million gallons a day or 1,350 second-feet May 31 (gage height, 7.10 feet); minimum, 2.4 million gallons a day or. 3.7 second-feet Oct. $25,26$.

1910, 1912-1929: Maximum discharge, 1,530 million gallons a day or 2,370 second-feet Dec. 24, 1927 (gage height, 9.38 feet); minimum, 1.4 million gallons a day or 2.2 second-feet Sept. 12-13, 1923.

REM ARKs. - Records good for ordinary stages except those estimated, which are poor; records for high stages poor. Anahola ditch diverts water 3 miles above station for irrigation and domestic supply.

Discharge, in million gallons a day, 1928-29

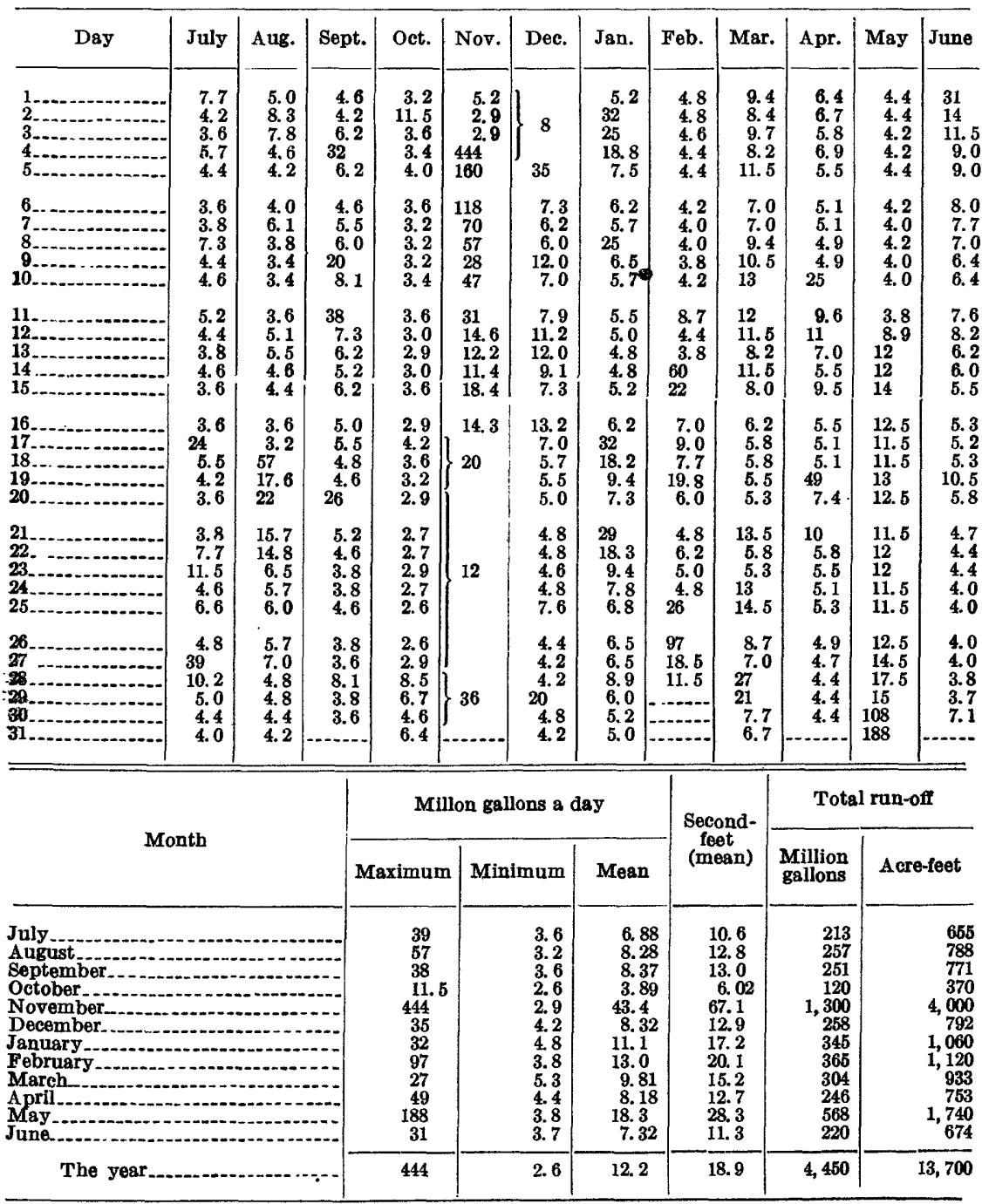




\section{ATAHOIA DTTCH ABOVE KANEHA RESBRVOIR, TEAB KHALIA, KAUAT}

Locatrion.-Water-stage recorder at upper end of second tunnel above Kaneha Reservoir, 5 miles northwest of Kealia.

Records Av AIL ABLE. - May, 1915, to June, 1929.

Extremes.- Maximum discharge during year, 80 million gallons a day or 124 second-feet May 31 (gage height, 4.68 feet); no flow several days when water was shut out of ditch.

1915-1929: Maximum discharge recorded, 130 million gallons a day or 201 second-feet Jan. 16, 1921 (gage height, 6.25 feet); no flow occasionally when water was shut out of ditch.

REMARKs.-Records good. This station measures water diverted from Anahola River to Kaneha Reservoir, where it is stored for irrigation. Regulated by head gates and spillways.

Discharge, in million gallons a day, 1928-29

\begin{tabular}{|c|c|c|c|c|c|c|c|c|c|c|c|c|}
\hline Day & July & Aug. & Sept. & Oct. & Nov. & Dec. & Jan. & Feb. & Mar. & Apr. & May & June \\
\hline $\begin{array}{l}1 \ldots \\
2 \ldots \\
3 \ldots \ldots \\
5 \ldots\end{array}$ & $\begin{array}{r}11.0 \\
3.7 \\
3.6 \\
6.6 \\
5.5\end{array}$ & $\begin{array}{r}11.0 \\
10.8 \\
7.0 \\
6.0 \\
4.5\end{array}$ & $\begin{array}{r}3.1 \\
3.4 \\
10.4 \\
17.8 \\
6.2\end{array}$ & $\begin{array}{l}3.0 \\
9.6 \\
3.9 \\
3.5 \\
6.1\end{array}$ & $\begin{array}{r}4.8 \\
2.2 \\
2.2 \\
10.7 \\
1.2\end{array}$ & $\begin{array}{l}0 \\
0 \\
0 \\
0 \\
0\end{array}$ & $\begin{array}{l}0 \\
0 \\
0 \\
0 \\
0\end{array}$ & $\begin{array}{l}1.9 \\
1.9 \\
1.8 \\
1.8 \\
1.8\end{array}$ & $\begin{array}{l}0 \\
0 \\
0 \\
0 \\
0\end{array}$ & $\begin{array}{l}0 \\
0 \\
0 \\
5.5 \\
4.1\end{array}$ & $\begin{array}{l}2.7 \\
3.3 \\
2.2 \\
2.3 \\
2.4\end{array}$ & $\begin{array}{l}0 \\
0 \\
4.4 \\
5.0 \\
5.8\end{array}$ \\
\hline $\begin{array}{r}6 \\
8 \\
8 \\
9\end{array}$ & $\begin{array}{r}3.1 \\
4.7 \\
10.0 \\
5.8 \\
6.0\end{array}$ & $\begin{array}{l}3.9 \\
8.8 \\
3.8 \\
3.2 \\
3.1\end{array}$ & $\begin{array}{r}4.2 \\
8.0 \\
6.6 \\
12.6 \\
10.9\end{array}$ & $\begin{array}{l}\text { 3. } 3 \\
\text { 3. } \\
3.2 \\
\text { 3. } \\
2.6\end{array}$ & $\begin{array}{l}1.1 \\
.9 \\
.3 \\
0 \\
0\end{array}$ & $\begin{array}{l}0 \\
0 \\
0 \\
0 \\
0\end{array}$ & $\begin{array}{l}0 \\
0 \\
0 \\
0 \\
0\end{array}$ & $\begin{array}{l}1.7 \\
1.7 \\
1.6 \\
1.5 \\
1.6\end{array}$ & $\begin{array}{r}0 \\
0 \\
1.4 \\
8.5 \\
11.8\end{array}$ & $\begin{array}{l}3.7 \\
3.2 \\
3.1 \\
4.7 \\
7.7\end{array}$ & $\begin{array}{l}1.8 \\
1.8 \\
2.5 \\
1.7 \\
1.6\end{array}$ & $\begin{array}{l}4.4 \\
3.9 \\
3.0 \\
2.6 \\
3.4\end{array}$ \\
\hline $\begin{array}{l}11 \\
12 \\
14 \\
15\end{array}$ & $\begin{array}{l}\text { 7. } 0 \\
\text { 5. } 8 \\
\text { 3. } 6 \\
\text { 5. } 5 \\
\text { 3. }\end{array}$ & $\begin{array}{l}3.3 \\
8.6 \\
6.4 \\
6.1 \\
4.5\end{array}$ & $\begin{array}{l}5.6 \\
4 \\
5.2 \\
4.5 \\
8.4\end{array}$ & $\begin{array}{l}2.9 \\
2.3 \\
2.2 \\
2.3 \\
3.4\end{array}$ & $\begin{array}{l}0 \\
0 \\
0 \\
0 \\
0\end{array}$ & $\begin{array}{l}0 \\
0 \\
0 \\
0 \\
0\end{array}$ & $\begin{array}{l}0.9 \\
2.9 \\
2.5 \\
1.1 \\
1.7\end{array}$ & $\begin{array}{l}5.6 \\
2.3 \\
1.8 \\
8.0 \\
5.4\end{array}$ & $\begin{array}{l}9.7 \\
6.4 \\
6.2 \\
7.6 \\
5.4\end{array}$ & $\begin{array}{l}0 \\
0 \\
1.6 \\
3.6 \\
8.9\end{array}$ & $\begin{array}{l}1.4 \\
1.2 \\
1.2 \\
1.2 \\
3.3\end{array}$ & $\begin{array}{l}5.7 \\
5.2 \\
3.1 \\
2.9 \\
2.3\end{array}$ \\
\hline $\begin{array}{l}16 \ldots \\
17 \\
18 \\
19 \\
20 \ldots\end{array}$ & $\begin{array}{r}4.4 \\
14.8 \\
6.9 \\
5.3 \\
3.7\end{array}$ & $\begin{array}{l}3.6 \\
4.2 \\
8.9 \\
0 \\
0\end{array}$ & $\begin{array}{r}4.4 \\
6.9 \\
5.4 \\
5.6 \\
14.2\end{array}$ & $\begin{array}{l}2.5 \\
\text { 5. } 8 \\
3.7 \\
3.0 \\
2.4\end{array}$ & $\begin{array}{l}0 \\
0 \\
0 \\
0 \\
0\end{array}$ & $\begin{array}{l}0 \\
0 \\
0 \\
0 \\
0\end{array}$ & $\begin{array}{l}3.5 \\
0 \\
0 \\
0 \\
.1\end{array}$ & $\begin{array}{r}7.0 \\
10.2 \\
6.2 \\
11.0 \\
4.6\end{array}$ & $\begin{array}{l}4.1 \\
3.5 \\
4.1 \\
3.1 \\
4.3\end{array}$ & $\begin{array}{r}4.1 \\
3.5 \\
3.4 \\
10.6 \\
0\end{array}$ & $\begin{array}{r}1.4 \\
1.1 \\
.9 \\
.9 \\
.8\end{array}$ & $\begin{array}{l}2.2 \\
2.3 \\
2.3 \\
\text { 6.8 } \\
2.6\end{array}$ \\
\hline 21 & $\begin{array}{r}5.8 \\
10.2 \\
12.2 \\
6.9 \\
11.1\end{array}$ & $\begin{array}{l}0 \\
0 \\
3.8 \\
4.4 \\
5.5\end{array}$ & $\begin{array}{l}6.1 \\
.02 \\
1.8 \\
3.0 \\
4.2\end{array}$ & $\begin{array}{l}2.2 \\
2.5 \\
2.2 \\
2.0 \\
1.8\end{array}$ & $\begin{array}{l}0 \\
0 \\
0 \\
0 \\
0\end{array}$ & $\begin{array}{l}0 \\
1.0 \\
2.5 \\
2.8 \\
8.5\end{array}$ & $\begin{array}{l}.5 \\
0 \\
0 \\
0\end{array}$ & $\begin{array}{l}3.4 \\
6.5 \\
3.4 \\
3.5 \\
9.8\end{array}$ & $\begin{array}{r}8.6 \\
4.6 \\
4.8 \\
9.8 \\
11.8\end{array}$ & $\begin{array}{l}0 \\
.05 \\
0 \\
2.3 \\
3.9\end{array}$ & $\begin{array}{r}.7 \\
.9 \\
1.1 \\
.8 \\
.8\end{array}$ & $\begin{array}{l}2.1 \\
2.0 \\
1.8 \\
1.8 \\
1.8\end{array}$ \\
\hline $\begin{array}{l}26 \\
27 \\
28 \\
30 \\
31\end{array}$ & $\begin{array}{l}7.4 \\
4.3 \\
0 \\
0 \\
1.5 \\
4.6\end{array}$ & $\begin{array}{l}4.9 \\
5.2 \\
3.3 \\
3.1 \\
2.9 \\
2.8\end{array}$ & $\begin{array}{l}3.8 \\
3.2 \\
8.1 \\
4.6 \\
3.5 \\
-2 .\end{array}$ & $\begin{array}{l}1.8 \\
2.4 \\
2.1 \\
4.9 \\
4.2 \\
5.6\end{array}$ & $\begin{array}{l}0 \\
0 \\
0 \\
0 \\
0\end{array}$ & $\begin{array}{l}3.1 \\
2.4 \\
2.4 \\
2.0 \\
0 \\
0\end{array}$ & $\begin{array}{l}0 \\
0 \\
0 \\
.8 \\
2.2 \\
2.0\end{array}$ & \begin{tabular}{l}
8.1 \\
0 \\
0 \\
\hdashline-1.2 \\
\hdashline
\end{tabular} & $\begin{array}{l}9.7 \\
5.4 \\
3.7 \\
0 \\
0 \\
0\end{array}$ & $\begin{array}{l}3.1 \\
2.6 \\
2.5 \\
2.4 \\
2.4\end{array}$ & $\begin{array}{r}1.2 \\
1.3 \\
4.4 \\
1.7 \\
10.7 \\
4.0\end{array}$ & $\begin{array}{l}2.1 \\
1.9 \\
1.7 \\
1.5 \\
1.5 \\
-\end{array}$ \\
\hline
\end{tabular}

\begin{tabular}{|c|c|c|c|c|c|c|}
\hline \multirow{2}{*}{ Month } & \multicolumn{3}{|c|}{ Million gallons a day } & \multirow{2}{*}{$\begin{array}{c}\text { Second- } \\
\text { feet } \\
\text { (mean) }\end{array}$} & \multicolumn{2}{|c|}{ Total run-off } \\
\hline & Maximum & Minlmum & Mean & & $\begin{array}{l}\text { Million } \\
\text { gallons }\end{array}$ & Acre-feet \\
\hline $\begin{array}{l}\text { July (29 days) } \\
\text { August (27 days) } \\
\text { September } \\
\text { Oetober } \\
\text { November (8 days) } \\
\text { December (8 days) } \\
\text { January (12 days) } \\
\text { February (26 days) } \\
\text { March (21 days) } \\
\text { April (22 days) } \\
\text { May - } 28 \text { days) } \\
\text { June (28) }\end{array}$ & $\begin{array}{r}14.8 \\
11.0 \\
17.8 \\
9.6 \\
10.7 \\
8.5 \\
3.5 \\
11.0 \\
11.8 \\
10.6 \\
10.7 \\
5.8\end{array}$ & 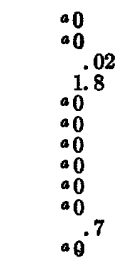 & $\begin{array}{l}\text { 6. } 38 \\
5.32 \\
6.20 \\
3.34 \\
2.92 \\
3.09 \\
1.55 \\
4.39 \\
6.40 \\
3.95 \\
2.04 \\
3.04\end{array}$ & $\begin{array}{l}9.87 \\
8.23 \\
9.59 \\
5.17 \\
4.52 \\
4.78 \\
2.40 \\
6.79 \\
9.90 \\
6.11 \\
3.16 \\
4.70\end{array}$ & $\begin{array}{c}185 \\
144 \\
186 \\
104 \\
23.4 \\
24.7 \\
18.6 \\
114 \\
134 \\
87.0 \\
63.3 \\
85.1\end{array}$ & $\begin{array}{r}\mathbf{5 6 8} \\
\mathbf{4 4 1} \\
\mathbf{5 7 1} \\
\mathbf{3 1 8} \\
\mathbf{7 2} \\
76 \\
\mathbf{5 7} \\
\mathbf{3 5 0} \\
\mathbf{4 1 2} \\
\mathbf{2 6 7} \\
\mathbf{1 9 4} \\
261\end{array}$ \\
\hline The year (273 days) & 17.8 & 0.0 & 4.28 & 6.62 & 1,170 & 3,590 \\
\hline
\end{tabular}

a The whole month considered. 
HANALET RIVER AT ELEVATION 625 FHET, HEAR HANALET, RAUAT

Locatron.-Water-stage recorder 2 miles west of Kauai Electric Co.'s power line and about 10 miles above mouth of stream.

Drainage area.-7.4 square miles.

Records av AILABLe. -January, 1914, to June, 1929.

ExTREMEs.-Maximum discharge during year, 3,440 million gallons a day or 5,320 second-feet Nov. 4 (gage height, 7.04 feet); minimum, 9.0 million gallons a day or 13.9 second-feet June 30 .

1914-1929: Maximum discharge, 6,500 million gallons a day or 10,100 second-feet Jan. 16, 1921 (gage height, 7.50 feet); minimum, 5.8 million gallons a day or 9.0 second-feet Apr. 28, May 1-3, 1926.

REMARKs. - Records good for ordinary stages except those estimated, which are poor; records for extremely high and low stages poor. Hanalei tunnel diverts water from stream about 2 miles above station.

Discharge, in million gallons a day, 1928-29

\begin{tabular}{|c|c|c|c|c|c|c|c|c|c|c|c|c|}
\hline & July & Aug. & Sept. & Oct. & Nov. & Dec. & Jan. & Feb. & Mar. & Apr. & May & June \\
\hline $\begin{array}{l}2 \\
8 \\
4 \\
5\end{array}$ & $\begin{array}{l}80 \\
26 \\
17.6 \\
22 \\
22\end{array}$ & $\begin{array}{l}42 \\
37 \\
30 \\
26 \\
24\end{array}$ & $\begin{array}{c}19.9 \\
20 \\
84 \\
100 \\
36\end{array}$ & $\begin{array}{r}20 \\
136 \\
24 \\
22 \\
22\end{array}$ & $\begin{array}{l}15.0 \\
13.6 \\
13.6 \\
681 \\
271\end{array}$ & $\begin{array}{l}19.5 \\
18.7 \\
18.0 \\
18.3 \\
40\end{array}$ & $\begin{array}{l}18.3 \\
19.1 \\
51 \\
39 \\
24\end{array}$ & $\begin{array}{l}13.3 \\
12.9 \\
12.6 \\
12.6 \\
12.6\end{array}$ & $\begin{array}{l}21 \\
43 \\
26 \\
81\end{array}$ & $\begin{array}{l}19.9 \\
21 \\
24 \\
23 \\
20\end{array}$ & $\begin{array}{l}28 \\
24 \\
15.8 \\
27 \\
18.0\end{array}$ & 26 \\
\hline $\begin{array}{r}6 \\
7 \\
8 \\
9 \\
9\end{array}$ & $\begin{array}{l}17.6 \\
24 \\
70 \\
30 \\
26\end{array}$ & $\begin{array}{l}22 \\
42 \\
22 \\
20 \\
19.5\end{array}$ & $\begin{array}{l}28 \\
30 \\
32 \\
46 \\
53\end{array}$ & \begin{tabular}{l|}
19.5 \\
19.5 \\
18.7 \\
17.6 \\
16.8
\end{tabular} & \begin{tabular}{|c|}
214 \\
129 \\
95 \\
110 \\
156
\end{tabular} & $\begin{array}{l}25 \\
19.9 \\
20 \\
31 \\
23\end{array}$ & $\begin{array}{l}20 \\
18.7 \\
19.1 \\
16.8 \\
16.1\end{array}$ & $\begin{array}{l}12.2 \\
11.9 \\
11.9 \\
11.9 \\
13.3\end{array}$ & $\begin{array}{l}34 \\
46 \\
49 \\
56 \\
66\end{array}$ & $\begin{array}{l}17.6 \\
16.5 \\
16.8 \\
16.8 \\
55\end{array}$ & $\begin{array}{l}14.7 \\
14.0 \\
13.6 \\
12.2 \\
12.2\end{array}$ & 17 \\
\hline$\frac{4}{3}-.$. & $\begin{array}{l}56 \\
30 \\
26 \\
24 \\
22\end{array}$ & $\begin{array}{l}18.7 \\
60 \\
30 \\
26 \\
23\end{array}$ & $\begin{array}{r}112 \\
32 \\
26 \\
24 \\
28\end{array}$ & $\begin{array}{l}16.5 \\
15.8 \\
16.1 \\
16.5 \\
15.4\end{array}$ & $\begin{array}{r}100 \\
46 \\
38 \\
38 \\
41\end{array}$ & $\begin{array}{l}24 \\
28 \\
34 \\
30 \\
24\end{array}$ & $\begin{array}{l}16.1 \\
15.0 \\
14.7 \\
14.7 \\
22\end{array}$ & $\begin{array}{l}39 \\
34 \\
16.1 \\
49 \\
69\end{array}$ & $\begin{array}{l}37 \\
30 \\
28 \\
27 \\
23\end{array}$ & $\begin{array}{l}31 \\
45 \\
28 \\
23 \\
35\end{array}$ & $\begin{array}{l}11.9 \\
11.2 \\
11.2 \\
12.9\end{array}$ & 22 \\
\hline 3 & $\begin{array}{l}25 \\
98 \\
42 \\
36 \\
26\end{array}$ & $\begin{array}{r}22 \\
48 \\
212 \\
90 \\
61\end{array}$ & $\begin{array}{r}22 \\
32 \\
35 \\
26 \\
137\end{array}$ & $\begin{array}{l}16.8 \\
18.0 \\
17.2 \\
18.3 \\
16.1\end{array}$ & $\begin{array}{l}39 \\
41 \\
52 \\
35 \\
28\end{array}$ & \begin{tabular}{|l|}
164 \\
35 \\
26 \\
22 \\
19.9
\end{tabular} & $\begin{array}{l}26 \\
87 \\
36 \\
20 \\
18.0\end{array}$ & $\begin{array}{l}91 \\
72 \\
33 \\
61 \\
28\end{array}$ & $\begin{array}{l}20 \\
18.7 \\
17.6 \\
16.8 \\
18.0\end{array}$ & \begin{tabular}{c|}
22 \\
19.1 \\
19.9 \\
168 \\
34
\end{tabular} & 15 & 15 \\
\hline $\begin{array}{l}1 \\
2 \\
3 \\
4 \\
6\end{array}$ & $\begin{array}{l}24 \\
41 \\
81 \\
30 \\
30\end{array}$ & \begin{tabular}{|c}
122 \\
80 \\
39 \\
32 \\
28
\end{tabular} & $\begin{array}{l}36 \\
25 \\
22 \\
21 \\
19.9\end{array}$ & $\begin{array}{l}15.4 \\
15.8 \\
14.7 \\
14.3 \\
13.6\end{array}$ & $\begin{array}{l}30 \\
46 \\
27 \\
23 \\
21\end{array}$ & $\begin{array}{l}18.7 \\
17.6 \\
16.8 \\
16.5 \\
62\end{array}$ & $\begin{array}{l}22 \\
21 \\
20 \\
16.5 \\
15.4\end{array}$ & $\begin{array}{l}22 \\
30 \\
21 \\
18.1 \\
28\end{array}$ & \begin{tabular}{|r}
38 \\
21 \\
25 \\
76 \\
109
\end{tabular} & \begin{tabular}{l|}
31 \\
24 \\
21 \\
19.5 \\
19.9
\end{tabular} & 12 & $\begin{array}{r}-14.0 \\
13.3 \\
12.6 \\
11.9 \\
11.9\end{array}$ \\
\hline 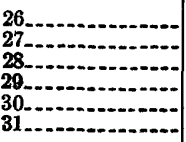 & $\begin{array}{r}26 \\
117 \\
124 \\
40 \\
30 \\
28\end{array}$ & $\begin{array}{l}25 \\
24 \\
21 \\
19.9 \\
19.9 \\
18.7\end{array}$ & $\begin{array}{l}19.1 \\
18.0 \\
24 \\
19.5 \\
18.0\end{array}$ & $\begin{array}{l}13.6 \\
14.0 \\
16.0 \\
31 \\
16.1 \\
16.8\end{array}$ & $\begin{array}{l}26 \\
20 \\
76 \\
26 \\
22\end{array}$ & \begin{tabular}{l|}
20 \\
17.2 \\
17.2 \\
89 \\
22 \\
19.1
\end{tabular} & $\begin{array}{l}15.0 \\
30 \\
22 \\
14.7 \\
14.0 \\
13.6\end{array}$ & \begin{tabular}{c}
40 \\
22 \\
27 \\
\hdashline$\ldots$ \\
\hdashline \\
\hdashline \\
\end{tabular} & $\begin{array}{l}33 \\
26 \\
35 \\
35 \\
24 \\
24\end{array}$ & $\begin{array}{l}17.6 \\
16.1 \\
15.4 \\
15.0 \\
16.0\end{array}$ & 55 & $\begin{array}{r}11 . \\
10 . \\
10 . \\
9 .\end{array}$ \\
\hline \multirow{2}{*}{\multicolumn{4}{|c|}{ Month }} & \multicolumn{4}{|c|}{ Million gallons a day } & \multirow{2}{*}{\multicolumn{2}{|c|}{$\begin{array}{c}\text { Second- } \\
\text { feet } \\
\text { (mean) }\end{array}$}} & \multicolumn{3}{|c|}{ Total run-of } \\
\hline & & & & Aaximum & n $\quad$ Min & imum & Mean & & & $\begin{array}{l}\text { Million } \\
\text { gallons }\end{array}$ & Acre & $\theta$-feet \\
\hline ly & $\ldots$ & - & & $\begin{array}{r}124 \\
212 \\
137 \\
136 \\
651 \\
164 \\
87 \\
91 \\
109 \\
168\end{array}$ & & \begin{tabular}{c}
17.6 \\
18.7 \\
18.0 \\
13.6 \\
18.6 \\
16.6 \\
18.6 \\
11.9 \\
16.8 \\
15.0 \\
\hdashline 9.3
\end{tabular} & $\begin{array}{l}41.7 \\
42,1 \\
38.2 \\
21.3 \\
81.4 \\
30.9 \\
23.1 \\
29.6 \\
35.9 \\
28.3 \\
21.1 \\
17.2\end{array}$ & \multicolumn{2}{|c|}{$\begin{array}{r}64.5 \\
65.1 \\
59.1 \\
33.0 \\
126 \\
47.8 \\
35.7 \\
45.6 \\
55.5 \\
43.8 \\
32.6 \\
26.6\end{array}$} & $\begin{array}{r}1,290 \\
1,300 \\
1,1150 \\
661 \\
2,440 \\
956 \\
715 \\
826 \\
1,110 \\
849 \\
655 \\
517\end{array}$ & \multicolumn{2}{|r|}{$\begin{array}{l}\mathbf{3}, 970 \\
\mathbf{4 , 0 1 0} \\
\mathbf{3}, 620 \\
\mathbf{2 , 0 3 0} \\
\mathbf{7 , 4 4 0} \\
\mathbf{2 ,}, 940 \\
2,200 \\
2,530 \\
\mathbf{3}, 420 \\
\mathbf{2 , 6 1 0} \\
\mathbf{2 , 0 1 0} \\
1,580\end{array}$} \\
\hline The year. & & & & 651 & & 9.3 & 34.2 & & 52.9 & 12,600 & & 38,300 \\
\hline
\end{tabular}

- Partly estimated. 
WAIOII STREAM NEAR HANALET, KAUAI

LOCATION.-Water-stage recorder $2 \frac{1}{2}$ miles south of Hanalei and 3 miles above mouth of stream.

Drainage area.-1.6 square miles.

RECORDS Available.-July, 1914, to June, 1929.

EXTREMES.-Maximum discharge during year, 920 million gallons a day or 1,420 second-feet Nov. 4 (gage height, 6.10 feet); minimum, 6.9 million gallons a day or 10.7 second-feet Feb. 10 .

1914-1929: Maximum discharge, 955 million gallons a day or 1,480 secondfeet Dec. 19, 1916 (gage height, 6.15 feet); minimum, 2.0 million gallons a day or 3.1 second-feet July 22, 1914.

REM ARKs. - Records good for ordinary stages except those estimated, which are fair; records for extremely high and low stages poor. No diversions.

Discharge, in million gallons à day, 1928-29

\begin{tabular}{|c|c|c|c|c|c|c|c|c|c|c|c|c|}
\hline Day & July & Aug. & Sept. & Oct. $\mathbf{N}$ & Nov. & Dec. & Jan. & Feb. & Mar. & Apr. & May & June \\
\hline & \begin{tabular}{l|}
56 \\
23 \\
17.0 \\
19.2 \\
18.0
\end{tabular} & $\begin{array}{l}37 \\
26 \\
18.0 \\
17.3 \\
16.0\end{array}$ & $\begin{array}{l}13.6 \\
16.4 \\
36 \\
73 \\
20\end{array}$ & \begin{tabular}{l|r}
10.9 & 1 \\
30 & 1 \\
13.6 & 1 \\
12.5 & 39 \\
16.4 & 20
\end{tabular} & $\begin{array}{l}16.0 \\
12.5 \\
13.9 \\
301 \\
207\end{array}$ & \begin{tabular}{r|}
8.9 \\
8.5 \\
8.7 \\
12.4 \\
33
\end{tabular} & $\begin{array}{l}10.5 \\
17.4 \\
41 \\
25 \\
14.7\end{array}$ & \begin{tabular}{l|}
7.5 \\
7.3 \\
7.3 \\
7.3 \\
7.2
\end{tabular} & $\begin{array}{l}14.7 \\
18.0 \\
27 \\
17.0 \\
42\end{array}$ & \begin{tabular}{l|}
7.9 \\
8.7 \\
9.1 \\
7.7 \\
7.5
\end{tabular} & $\begin{array}{r}7.9 \\
19.5 \\
10.2 \\
23 \\
17.4\end{array}$ & $\begin{array}{l}13.6 \\
11.2 \\
10.7 \\
13.9 \\
18.0\end{array}$ \\
\hline $\begin{array}{r}6 \ldots . \\
7 \\
8 \ldots \\
9 \ldots \\
10 \ldots\end{array}$ & $\begin{array}{l}13.9 \\
29 \\
50 \\
22 \\
24\end{array}$ & $\begin{array}{l}\text { 13. } 6 \\
14.7 \\
11.7 \\
11.2 \\
11.9\end{array}$ & $\begin{array}{l}16.7 \\
23 \\
20 \\
32 \\
32\end{array}$ & \begin{tabular}{r|r}
11.9 & 12 \\
12.7 & 9 \\
12.2 & 6 \\
10.9 & 5 \\
10.2 & 10
\end{tabular} & $\begin{array}{r}129 \\
93 \\
60 \\
57 \\
104\end{array}$ & $\begin{array}{l}17.0 \\
12.5 \\
14.7 \\
39 \\
21\end{array}$ & \begin{tabular}{l|}
13.3 \\
16.3 \\
24 \\
13.9 \\
12.2
\end{tabular} & $\begin{array}{l}7.2 \\
7.2 \\
7.2 \\
7.2 \\
7.3\end{array}$ & $\begin{array}{l}18.0 \\
21 \\
24 \\
37 \\
29\end{array}$ & \begin{tabular}{r|}
7.5 \\
7.3 \\
7.5 \\
12.7 \\
41
\end{tabular} & $\begin{array}{r}11.7 \\
10.9 \\
12.7 \\
9.1 \\
10.0\end{array}$ & $\begin{array}{r}16.4 \\
11.9 \\
9.1 \\
8.7 \\
10.0\end{array}$ \\
\hline 5 & $\begin{array}{l}44 \\
22 \\
18.2 \\
20 \\
21\end{array}$ & $\begin{array}{l}13.9 \\
31 \\
19.2 \\
17.6 \\
16.8\end{array}$ & $\begin{array}{l}46 \\
17.3 \\
13.6 \\
13.0 \\
16.0\end{array}$ & $\begin{array}{r}9.7 \\
9.7 \\
9.7 \\
11.4 \\
11.9\end{array}$ & $\begin{array}{l}59 \\
21 \\
24 \\
29 \\
25\end{array}$ & $\begin{array}{l}19.3 \\
20 \\
26 \\
21 \\
15.7\end{array}$ & $\begin{array}{l}11.7 \\
11.2 \\
10.9 \\
10.9 \\
22\end{array}$ & \begin{tabular}{l|}
24 \\
18.6 \\
11.7 \\
15.4 \\
55
\end{tabular} & $\begin{array}{l}18.4 \\
15.7 \\
14.7 \\
17.1 \\
13.6\end{array}$ & \begin{tabular}{l|}
19.6 \\
52 \\
18.8 \\
21 \\
23
\end{tabular} & $\begin{array}{r}8.9 \\
7.9 \\
8.7 \\
11.7 \\
15.9\end{array}$ & $\begin{array}{l}26 \\
17.7 \\
13.6 \\
12.2 \\
9.1\end{array}$ \\
\hline 16 & $\begin{array}{l}28 \\
75 \\
24 \\
18.4 \\
14.4\end{array}$ & $\begin{array}{l}14.1 \\
27 \\
88 \\
33 \\
29\end{array}$ & $\begin{array}{l}12.7 \\
14.4 \\
12.2 \\
12.5 \\
24\end{array}$ & $\begin{array}{l}11.2 \\
15.0 \\
15.7 \\
13.9 \\
11.9\end{array}$ & \begin{tabular}{l|}
21 \\
30 \\
48 \\
18.0 \\
14.1
\end{tabular} & \begin{tabular}{l|}
24 \\
13.9 \\
11.9 \\
10.2 \\
9.5
\end{tabular} & 10.0 & \begin{tabular}{l|}
72 \\
32 \\
19.1 \\
24 \\
14.7
\end{tabular} & $\begin{array}{r}10.7 \\
9.3 \\
8.5 \\
8.1 \\
9.1\end{array}$ & $\begin{array}{l}13.3 \\
10.5 \\
15.7 \\
38 \\
15.4\end{array}$ & $\begin{array}{l}9.5 \\
8.3 \\
77.9 \\
7.3 \\
7.3\end{array}$ & $\begin{array}{r}9.7 \\
9.3 \\
8.5 \\
12.3 \\
11.9\end{array}$ \\
\hline 21.22 & $\begin{array}{l}14.4 \\
22 \\
58 \\
18.8 \\
29\end{array}$ & $\begin{array}{l}25 \\
23 \\
14.4 \\
13.3 \\
15.0\end{array}$ & $\begin{array}{r}13.6 \\
11.2 \\
10.5 \\
10.0 \\
9.5\end{array}$ & $\begin{array}{l}13.0 \\
15.0 \\
12.5 \\
11.4 \\
11.2\end{array}$ & $\begin{array}{l}30 \\
54 \\
17.0 \\
11.9 \\
10.2\end{array}$ & \begin{tabular}{r|}
9.5 \\
9.1 \\
8.9 \\
14.8 \\
37
\end{tabular} & \begin{tabular}{l|}
25 \\
17.5 \\
14.7 \\
10.5 \\
9.1
\end{tabular} & \begin{tabular}{l|}
12.2 \\
24 \\
13.0 \\
10.2 \\
10.1
\end{tabular} & $\begin{array}{l}34 \\
12.2 \\
11.9 \\
13.0 \\
16.1\end{array}$ & $\begin{array}{r}11.9 \\
10.2 \\
9.1 \\
8.5 \\
8.3\end{array}$ & $\begin{array}{l}7.2 \\
7.5 \\
9.1 \\
7.5 \\
7.3\end{array}$ & $\begin{array}{r}9.3 \\
7.9 \\
7.3 \\
7.2 \\
10.2\end{array}$ \\
\hline $\begin{array}{l}26 \\
27 \\
28 \\
31\end{array}$ & $\begin{array}{l}33 \\
136 \\
47 \\
23 \\
17.7 \\
22\end{array}$ & $\begin{array}{l}14.7 \\
12.5 \\
11.7 \\
10.9 \\
10.7 \\
10.7\end{array}$ & $\begin{array}{r}9.5 \\
9.5 \\
13.3 \\
13.6 \\
13.8\end{array}$ & \begin{tabular}{l|l}
11.2 & \\
12.7 & \\
11.4 & \\
15.8 & \\
11.9 & \\
18.2 & $\ldots$
\end{tabular} & $\begin{array}{r}9.5 \\
8.9 \\
11.9 \\
99.5 \\
9.1\end{array}$ & $\begin{array}{l}13.0 \\
11.2 \\
14.0 \\
28 \\
12.7 \\
11.2\end{array}$ & $\begin{array}{r}8.7 \\
11.0 \\
14.3 \\
8.9 \\
7.9 \\
7.5\end{array}$ & $\begin{array}{l}28 \\
14.7 \\
16.7\end{array}$ & $\begin{array}{r}9.3 \\
7.9 \\
8.2 \\
16.7 \\
10.0 \\
8.5\end{array}$ & $\begin{array}{l}8.3 \\
7.9 \\
7.9 \\
7.7 \\
7.9 \\
-9\end{array}$ & $\begin{array}{l}8.9 \\
22 \\
24.1 \\
198 \\
38 \\
19.2\end{array}$ & $\begin{array}{r}11.2 \\
8.3 \\
7.2 \\
7.0 \\
6.7\end{array}$ \\
\hline \multirow{2}{*}{\multicolumn{4}{|c|}{ Month }} & \multicolumn{6}{|c|}{ Million gallons a day } & \multicolumn{3}{|c|}{ Total run-off } \\
\hline & & & & Maximum & $\begin{array}{l}n \\
\text { a Min }\end{array}$ & imum & Mear & \multicolumn{2}{|c|}{$\begin{array}{c}\text { Second- } \\
\text { feet } \\
\text { (mean) }\end{array}$} & $\begin{array}{l}\text { Million } \\
\text { gallons }\end{array}$ & \multicolumn{2}{|c|}{ Acre-feet } \\
\hline \multicolumn{4}{|c|}{ 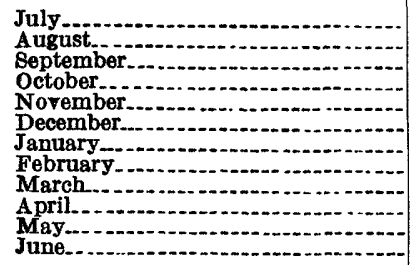 } & $\begin{array}{r}136 \\
88 \\
73 \\
30 \\
391 \\
39 \\
41 \\
72 \\
42 \\
52 \\
38 \\
26\end{array}$ & $\begin{array}{l}6 \\
8 \\
3 \\
0 \\
1 \\
9 \\
1 \\
2 \\
2 \\
2 \\
2 \\
8 \\
6\end{array}$ & $\begin{aligned} 13.9 \\
10.7 \\
9.5 \\
9.7 \\
8.9 \\
8.5 \\
7.5 \\
7.2 \\
7.9 \\
7.3 \\
7.2 \\
6.7\end{aligned}$ & & & $\begin{array}{l}48.7 \\
31.4 \\
29.9 \\
20.3 \\
79.7 \\
25.8 \\
24.8 \\
26.9 \\
26.0 \\
22.3 \\
19.8 \\
17.3\end{array}$ & $\begin{array}{r}978 \\
629 \\
579 \\
406 \\
1,540 \\
517 \\
496 \\
488 \\
521 \\
432 \\
396 \\
336\end{array}$ & & $\begin{array}{l}3,000 \\
1,930 \\
1,780 \\
1,250 \\
4,740 \\
1,590 \\
1,520 \\
1,500 \\
1,600 \\
1,330 \\
1,220 \\
1,030\end{array}$ \\
\hline & 391 & & 6.7 & & 0.1 & 31.1 & 7,320 & & 22,500 \\
\hline
\end{tabular}


LUMAFAI RIVER WEAR HAWALEI, KAUAI

Location.-Water-stage recorder 6 miles above mouth and 4132 miles southwest of Hanalei.

Drainage aRea. - 7.1 square miles.

Records Avatlable.-May, 1914, to October, 1917; July, 1920, to June, 1929.

Extremes.-Maximum discharge during year, 3,290 million gallons a day or 5,090 second-feet Nov. 4 (gage height, 6.99 feet); minimum, 14.9 million gallons a day or 23.1 second-feet June 29 and 30 .

1914-1917, 1920-1929: Maximum discharge (estimated), 5,000 million gallons a day or 7,740 second-feet Sept. 11, 1922 (gage height, 9.41 feet); minimum, 13.6 million gallons a day or 21 second feet May 15, 17, 1926.

REMARKs. - Records good for ordinary stages, fair for estimated period, poor for extremely high stages. No diversions.

Discharge, in million gallons a day, 1928-29

\begin{tabular}{|c|c|c|c|c|c|c|c|c|c|c|c|c|}
\hline Day & July & Aug. & Sept. & Oct. & fov. & Dec. & Jan. & Feb. & Mar. & Apr. & May & June \\
\hline $\begin{array}{l}3 \ldots \\
4 \ldots \\
5 \ldots\end{array}$ & $\begin{array}{r}184 \\
68 \\
42 \\
47 \\
47\end{array}$ & $\begin{array}{l}94 \\
68 \\
49 \\
44 \\
41\end{array}$ & $\begin{array}{r}31 \\
38 \\
136 \\
305 \\
76\end{array}$ & \begin{tabular}{r|r}
27 & \\
151 & \\
40 & \\
34 \\
41
\end{tabular} \mid${ }^{a} 1$, & $\begin{array}{r}24 \\
24 \\
25 \\
750 \\
750\end{array}$ & $\begin{array}{r}30 \\
29 \\
28 \\
40 \\
129\end{array}$ & $\begin{array}{r}30 \\
41 \\
181 \\
92 \\
48\end{array}$ & $\begin{array}{l}24 \\
23 \\
22 \\
22 \\
21\end{array}$ & $\begin{array}{r}45 \\
52 \\
200 \\
96 \\
376\end{array}$ & $\begin{array}{l}25 \\
26 \\
26 \\
24 \\
22\end{array}$ & $\begin{array}{l}21 \\
24 \\
24 \\
43 \\
42\end{array}$ & $\begin{array}{l}66 \\
30 \\
27 \\
31 \\
42\end{array}$ \\
\hline $\begin{array}{r}6 .- \\
7 .- \\
8 \ldots \\
9- \\
10 .\end{array}$ & $\begin{array}{r}35 \\
108 \\
355 \\
79 \\
70\end{array}$ & $\begin{array}{l}37 \\
50 \\
34 \\
31 \\
32\end{array}$ & $\begin{array}{l}52 \\
83 \\
65 \\
74 \\
76\end{array}$ & $\begin{array}{l}31 \\
30 \\
30 \\
27 \\
26\end{array}$ & $\begin{array}{l}750 \\
398 \\
274 \\
392 \\
604\end{array}$ & $\begin{array}{r}75 \\
47 \\
55 \\
134 \\
78\end{array}$ & $\begin{array}{l}38 \\
35 \\
47 \\
30 \\
29\end{array}$ & $\begin{array}{l}21 \\
21 \\
20 \\
19.9 \\
20\end{array}$ & $\begin{array}{l}114 \\
160 \\
174 \\
186 \\
123\end{array}$ & $\begin{array}{r}22 \\
21 \\
21 \\
50 \\
277\end{array}$ & $\begin{array}{l}22 \\
27 \\
40 \\
24 \\
24\end{array}$ & $\begin{array}{l}35 \\
26 \\
20 \\
18.9 \\
19.9\end{array}$ \\
\hline 14 & $\begin{array}{r}185 \\
82 \\
59 \\
73 \\
67\end{array}$ & $\begin{array}{r}34 \\
a 58\end{array}$ & $\begin{array}{r}213 \\
59 \\
44 \\
39 \\
48\end{array}$ & $\begin{array}{l}25 \\
24 \\
23 \\
24 \\
24\end{array}$ & $\begin{array}{r}326 \\
87 \\
83 \\
76 \\
67\end{array}$ & $\begin{array}{l}65 \\
85 \\
71 \\
64 \\
49\end{array}$ & $\begin{array}{l}27 \\
26 \\
25 \\
26 \\
82\end{array}$ & $\begin{array}{r}96 \\
89 \\
50 \\
59 \\
235\end{array}$ & $\begin{array}{l}71 . \\
54 \\
50 \\
48 \\
43\end{array}$ & $\begin{array}{r}101 \\
153 \\
\times 74 \\
\\
55\end{array}$ & $\begin{array}{l}21 \\
19.4 \\
19.9 \\
24 \\
30\end{array}$ & $\begin{array}{l}47 \\
32 \\
24 \\
22 \\
18.9\end{array}$ \\
\hline 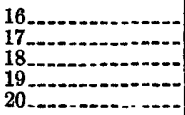 & $\begin{array}{r}129 \\
428 \\
106 \\
63 \\
48\end{array}$ & 340 & $\begin{array}{r}37 \\
44 \\
41 \\
42 \\
107\end{array}$ & $\begin{array}{l}24 \\
30 \\
28 \\
29 \\
24\end{array}$ & $\begin{array}{r}64 \\
127 \\
154 \\
64 \\
46\end{array}$ & $\begin{array}{r}105 \\
51 \\
39 \\
34 \\
31\end{array}$ & $\begin{array}{r}104 \\
100 \\
56 \\
40 \\
34\end{array}$ & $\begin{array}{r}340 \\
209 \\
84 \\
145 \\
73\end{array}$ & $\begin{array}{l}35 \\
32 \\
30 \\
27 \\
29\end{array}$ & $\begin{array}{r}a 36 \\
42 \\
214 \\
53\end{array}$ & $\begin{array}{l}21 \\
19.4 \\
18.4 \\
17.8 \\
17,3\end{array}$ & $\begin{array}{l}19.4 \\
17.8 \\
17.3 \\
17.8 \\
17.8\end{array}$ \\
\hline 23 & $\begin{array}{r}42 \\
59 \\
131 \\
50 \\
66\end{array}$ & 80 & $\begin{array}{l}44 \\
34 \\
30 \\
28 \\
27\end{array}$ & $\begin{array}{l}25 \\
31 \\
26 \\
23 \\
22\end{array}$ & $\begin{array}{r}102 \\
150 \\
54 \\
40 \\
34\end{array}$ & $\begin{array}{r}29 \\
27 \\
26 \\
36 \\
302\end{array}$ & $\begin{array}{l}75 \\
68 \\
80 \\
42 \\
34\end{array}$ & $\begin{array}{r}53 \\
136 \\
55 \\
40 \\
36\end{array}$ & $\begin{array}{r}127 \\
43 \\
39 \\
55 \\
87\end{array}$ & $\begin{array}{l}39 \\
32 \\
30 \\
27 \\
26\end{array}$ & $\begin{array}{l}16.8 \\
17.8 \\
19.9 \\
16.8 \\
16.2\end{array}$ & $\begin{array}{l}17.3 \\
16.2 \\
15.3 \\
16.2 \\
19.4\end{array}$ \\
\hline - & $\begin{array}{r}69 \\
384 \\
266 \\
73 \\
53 \\
65\end{array}$ & $\begin{array}{r}32 \\
\\
\times 28 \\
27 \\
27\end{array}$ & $\begin{array}{l}27 \\
26 \\
36 \\
33 \\
35\end{array}$ & $\begin{array}{l}21 \\
22 \\
21 \\
31 \\
23 \\
24\end{array}$ & $\begin{array}{c}31 \\
29 \\
54 \\
32 \\
30 \\
-\end{array}$ & $\begin{array}{l}62 \\
40 \\
38 \\
89 \\
38 \\
32\end{array}$ & $\begin{array}{l}30 \\
30 \\
44 \\
51 \\
30 \\
27 \\
25\end{array}$ & $\begin{array}{l}65 \\
47 \\
53\end{array}$ & $\begin{array}{l}37 \\
30 \\
32 \\
37 \\
28 \\
27\end{array}$ & $\begin{array}{l}24 \\
22 \\
22 \\
21 \\
21 \\
\\
\end{array}$ & $\begin{array}{c}18.4 \\
32 \\
37 \\
90 \\
115 \\
46\end{array}$ & $\begin{array}{r}19.9 \\
16.8 \\
15.7 \\
15.3 \\
15.3 \\
\hdashline-.-\end{array}$ \\
\hline \multirow{2}{*}{\multicolumn{4}{|c|}{ Month }} & \multicolumn{4}{|c|}{ Million gallons a day } & \multirow{2}{*}{\multicolumn{2}{|c|}{$\begin{array}{l}\text { Second- } \\
\text { feet } \\
\text { (mean) }\end{array}$}} & \multicolumn{3}{|c|}{ Total run-off } \\
\hline & & & & Iaximum & Min & um & Mean & & & $\begin{array}{l}\text { Million } \\
\text { gallons }\end{array}$ & Acr & feet \\
\hline $\begin{array}{l}\text { July } \\
\text { August } \\
\text { September } \\
\text { October } \\
\text { November } \\
\text { December } \\
\text { January } \\
\text { February } \\
\text { March } \\
\text { A pril } \\
\text { May } \\
\text { June }\end{array}$ & $\ldots$ & & & $\begin{array}{r}428 \\
3305 \\
151 \\
1,750 \\
302 \\
181 \\
340 \\
376 \\
277 \\
115 \\
66\end{array}$ & & $\begin{array}{l}35 \\
-26 \\
21 \\
24 \\
26 \\
25 \\
19.9 \\
27 \\
21 \\
16.2 \\
15.3\end{array}$ & $\begin{array}{r}114 \\
90 . y \\
64.3 \\
31.0 \\
221.0 \\
63.2 \\
51.5 \\
74.4 \\
80.2 \\
53.9 \\
29.8 \\
23.9\end{array}$ & \multicolumn{2}{|c|}{$\begin{array}{c}176 \\
141 \\
99.5 \\
48.0 \\
342 \\
97.8 \\
79.7 \\
115 \\
124 \\
83.4 \\
46.1 \\
37.0\end{array}$} & $\begin{array}{r}3,530 \\
2,820 \\
1,930 \\
961 \\
6,640 \\
1,960 \\
1,600 \\
2,080 \\
2,490 \\
1,620 \\
925 \\
717\end{array}$ & \multicolumn{2}{|r|}{$\begin{array}{r}10,800 \\
8,650 \\
5,920 \\
2,950 \\
20,300 \\
6,010 \\
4,900 \\
6,390 \\
7,630 \\
4,960 \\
2,840 \\
2,200\end{array}$} \\
\hline \multicolumn{2}{|l|}{ The year... } & - & & 1,750 & & 15.3 & 74. & & 116 & 27,300 & & 83,600 \\
\hline
\end{tabular}

- Partly estimated.

$67754-32-3$ 


\section{MISCELLANEOUS MEASUREMENTS}

Measurements of streams and ditches on the island of Kauai at other than regular gaging stations are listed below:

Miscellaneous discharge measurements on Kauai, 1928-29

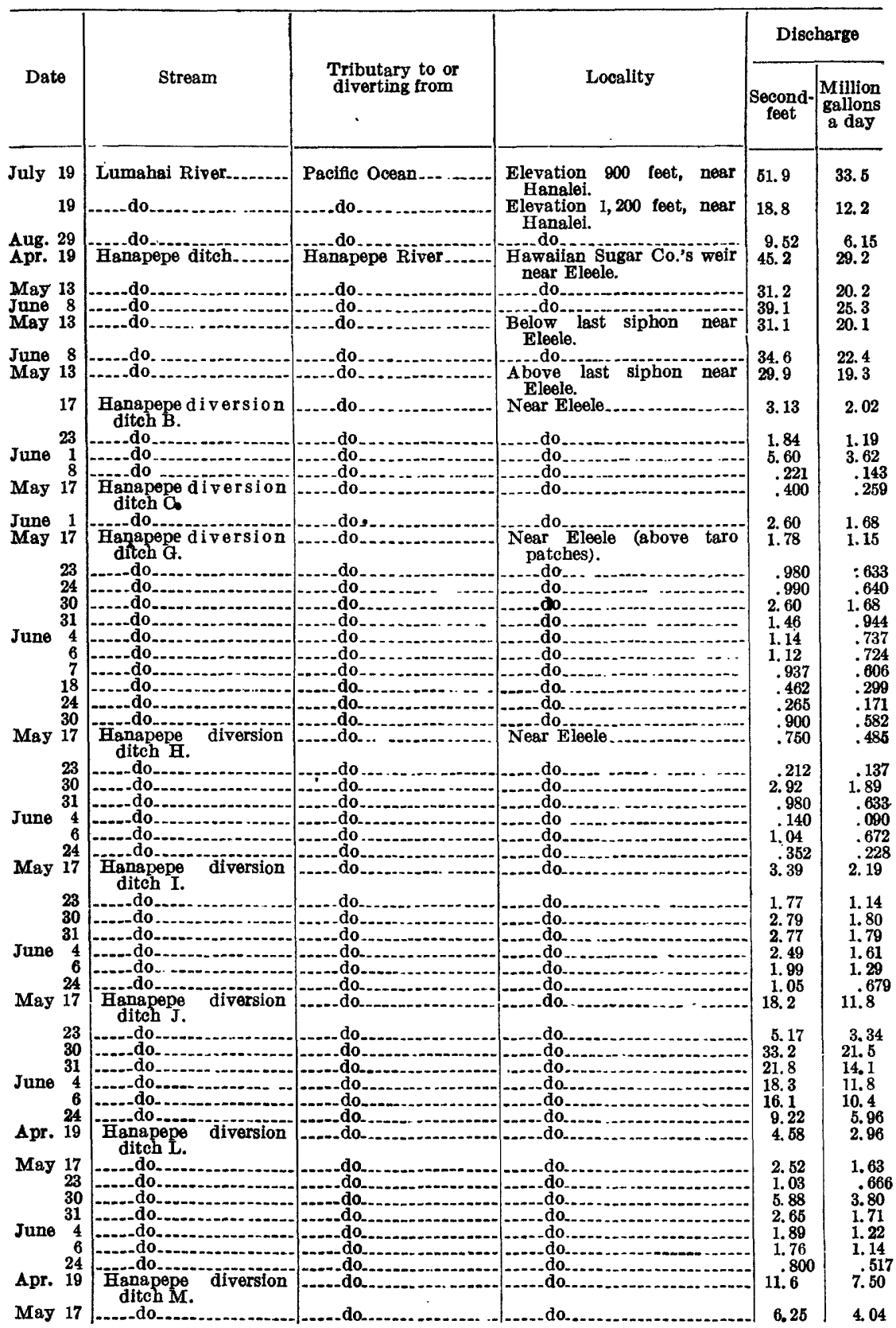


Miscellaneous discharge measurements on $K$ auai, 1928-29-Continued

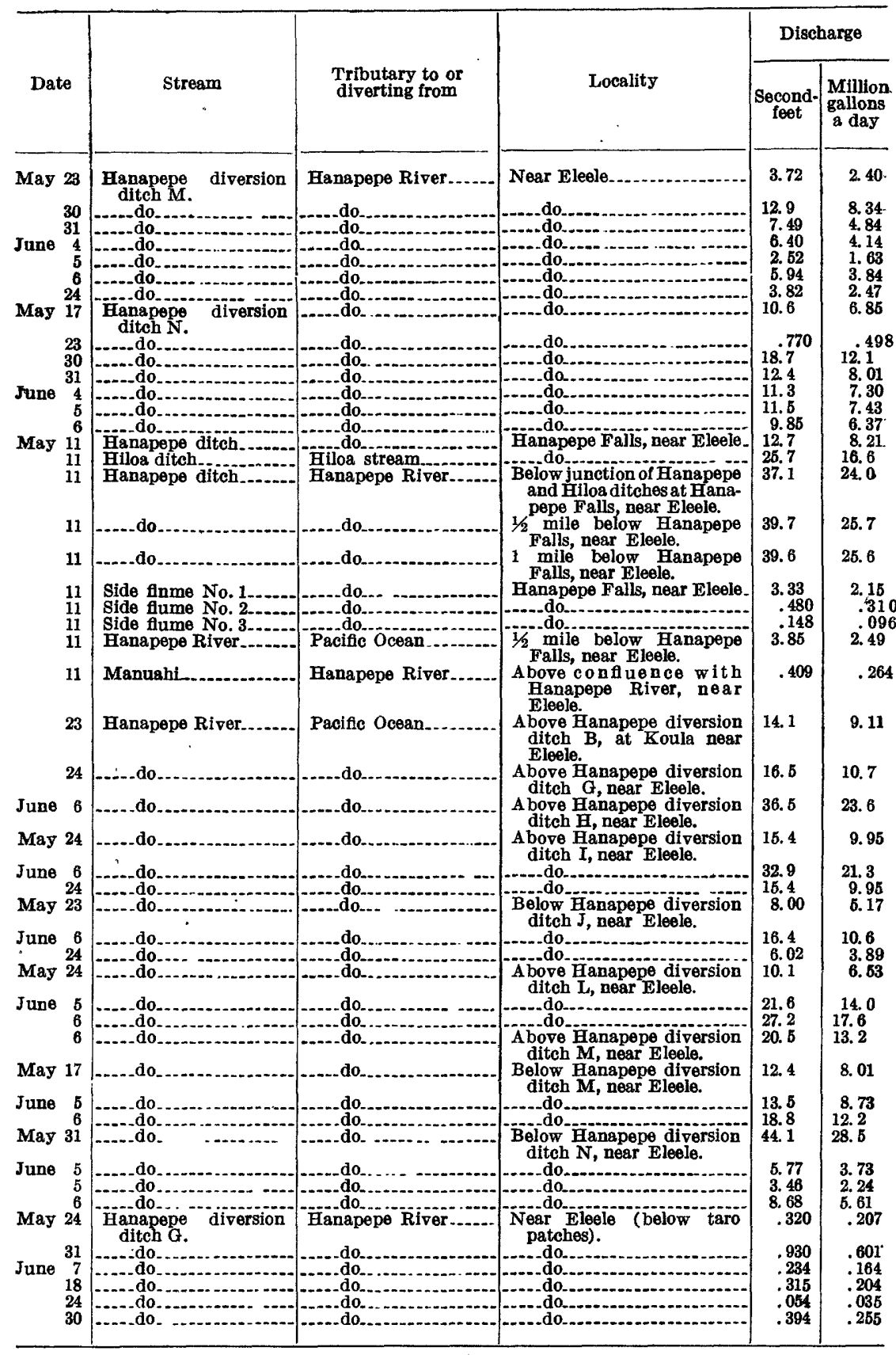




\section{ISLAND OF OAHU}

\section{RIGHT BRATCH OF NORTH FORK OF KAUKONAHOA STREAM NEAR WAHIAWA, OAHU}

Location.-Water-stage recorder 200 feet upstream from intake of Wahiawa Water Co.'s tunnel, which is just below confluence of right and left branches of North Fork of Kaukonahua Stream and 8 miles northeast of Wahiawa.

Drain Age AREA.- - 1.2 square miles.

RECORDS AVAILABLE. - May, 1913, to June, 1929.

ExTREMEs.-Maximum discharge during year, 754 million gallons a day or 1,170 second-feet Oct. 1 (gage height, 7.47 feet); minimum, 0.4 million gallons a day or 0.6 second-foot Feb. 11, 12 .

1913-1929: Maximum discharge, about 985 million gallons a day or 1,520 second-feet Mar. 26, 1920 (gage height, 9.0 feet, determined from floodmarks and comparison with record of left branch of North Fork of this stream); minimum, 0.09 million gallons a day or 0.15 second-foot Mar. 22, 1926.

REMARKs.-Records good for ordinary stages; poor for high stages and estimated the periods. No diversions above station.

Discharge, in million gallons a day, 1928-29

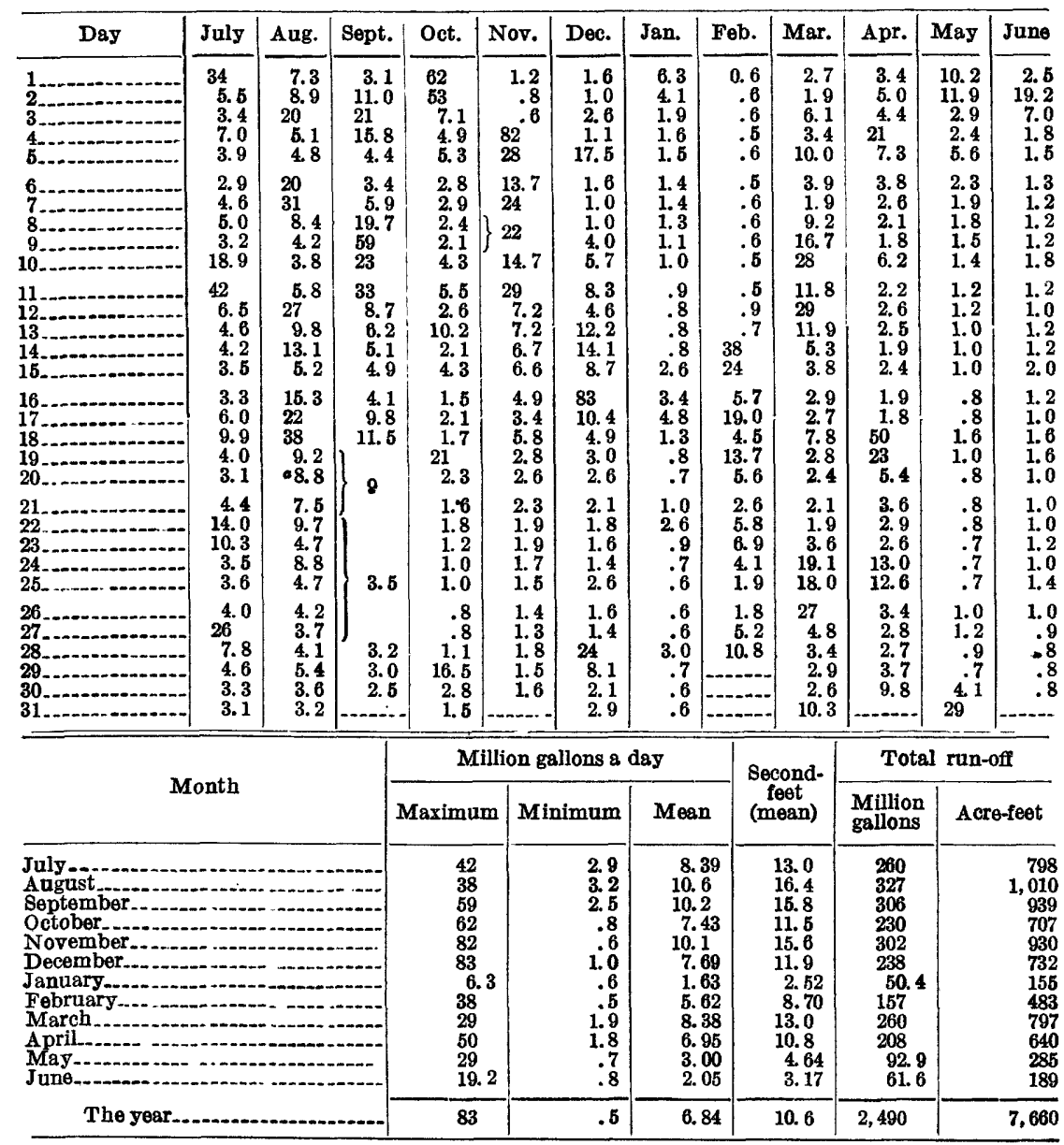

- Partly estimated. 


\section{IBFT BRANCH OF NORTH FORK OF KAUKONAFUA STREAM NBAR WAHAWA, OAHU}

Loisation.-Water-stage recorder 100 feet upstream from intake of Wahiawa Water Co.'s tunnel, which is just below confluence of the right and left branches of North Fork of Kaukonahua Stream, and 8 miles northeast of Wahiawa.

Drainagi area.-1.5 square miles.

RicoRDs AVAILABLE.-May, 1913, to June, 1929.

Ex'rREMes.-Maximum discharge during year, 2,540 million gallons a day or 3,930 second-feet Oct. 1 (gage height, 8.78 feet); minimum, 0.5 million gallons a day or 0.8 second-foot June 30 .

1913-1929: Maximum discharge, about 4,080 million gallons a day or 6,310 second-feet Jan. 14, 1923 (gage height, 10.3 feet); minimum, 0.1 million gallons a day or 0.16 second-foot Feb. 18, Mar. 5, 1920 .

REMARKs.- Records fair for ordinary stages; poor for high stages and estimated periods. No diversions above station.

Discharge, in million gallons a day, 1928-29

\begin{tabular}{|c|c|c|c|c|c|c|c|c|c|c|c|c|}
\hline Day & July & Aug. & Sept. & Oct. & Nov. & Dec. & Jan. & Feb. & Mar. & Apr. & May & June \\
\hline $\begin{array}{l}1 \\
2 \ldots \\
3 \\
4 \ldots \\
5\end{array}$ & \begin{tabular}{r}
\multicolumn{1}{c}{36} \\
8.5 \\
5.1 \\
14.2 \\
5.3
\end{tabular} & $\begin{array}{l}22 \\
25 \\
33 \\
12.5 \\
13.4\end{array}$ & $\begin{array}{c}6.2 \\
23 \\
27 \\
28 \\
8.9\end{array}$ & $\left\{\begin{array}{l}145 \\
100 \\
10\end{array}\right.$ & $\begin{array}{r}3.6 \\
3.0 \\
2.9 \\
95 \\
26\end{array}$ & $\begin{array}{r}3.0 \\
2.4 \\
4.1 \\
2.6 \\
13.8\end{array}$ & $\begin{array}{l}8.0 \\
4.9 \\
2.8 \\
2.5 \\
2.4\end{array}$ & $\begin{array}{l}1.2 \\
1.2 \\
1.2 \\
1.1 \\
1.1\end{array}$ & $\begin{array}{r}3.8 \\
3.9 \\
5.9 \\
5.3 \\
10.2\end{array}$ & $\begin{array}{r}4.1 \\
5.4 \\
7.4 \\
14.3 \\
7.2\end{array}$ & $\begin{array}{l}7.3 \\
8.8 \\
3.2 \\
2.8 \\
6.1\end{array}$ & $\begin{array}{l}2.4 \\
9.4 \\
4.3 \\
2.3 \\
2.1\end{array}$ \\
\hline $\begin{array}{r}6 \ldots \\
7 .- \\
8 \\
9 \\
10 .-\end{array}$ & $\begin{array}{r}4.4 \\
6.6 \\
10.7 \\
6.4 \\
35\end{array}$ & $\begin{array}{r}12.6 \\
15.2 \\
6.9 \\
6.2 \\
6.2\end{array}$ & $\begin{array}{l}6.4 \\
11.6 \\
16.1 \\
69 \\
43\end{array}$ & 5. 5 & $\begin{array}{c}15.6 \\
25 \\
9.7 \\
43 \\
17.0\end{array}$ & $\begin{array}{r}3.0 \\
2.3 \\
2.2 \\
5.9 \\
12.0\end{array}$ & $\begin{array}{l}2.4 \\
2.2 \\
2.2 \\
1.9 \\
1.9\end{array}$ & $\begin{array}{l}1.1 \\
1.1 \\
1.0 \\
1.0 \\
1.0\end{array}$ & $\begin{array}{r}5.8 \\
3.0 \\
11.1 \\
25 \\
24\end{array}$ & $\begin{array}{l}4.8 \\
4.9 \\
3.4 \\
2.6 \\
9.3\end{array}$ & $\begin{array}{l}3.0 \\
2.7 \\
2.5 \\
2.2 \\
2.1\end{array}$ & $\begin{array}{l}1.8 \\
1.7 \\
4.1 \\
2.1 \\
2.9\end{array}$ \\
\hline $\begin{array}{l}11 \ldots \\
12 \\
13 \\
14 \\
15\end{array}$ & \begin{tabular}{r}
\multicolumn{1}{c}{56} \\
14.3 \\
9.2 \\
8.6 \\
6.9
\end{tabular} & $\begin{array}{l}13.0 \\
24 \\
22 \\
24 \\
8.9\end{array}$ & $\begin{array}{r}41 \\
12.0 \\
9.5 \\
8.6 \\
8.9\end{array}$ & 11 & $\begin{array}{l}36 \\
10.3 \\
11.1 \\
11.6 \\
13.2\end{array}$ & $\begin{array}{l}16.7 \\
10.4 \\
13.2 \\
26 \\
16.1\end{array}$ & $\begin{array}{l}1.9 \\
1.8 \\
1.7 \\
1.8 \\
2.5\end{array}$ & $\begin{array}{r}.9 \\
1.2 \\
1.1 \\
13.4 \\
10.9\end{array}$ & $\begin{array}{r}13.5 \\
41 \\
11.0 \\
6.6 \\
5.6\end{array}$ & $\begin{array}{l}3.2 \\
4.7 \\
5.6 \\
2.8 \\
5.5\end{array}$ & $\begin{array}{l}2.0 \\
1.9 \\
1.8 \\
1.8 \\
1.9\end{array}$ & $\begin{array}{l}1.6 \\
2.9 \\
3.2 \\
7.1 \\
\text { 5. } 3\end{array}$ \\
\hline $\begin{array}{l}16 \\
17 \\
18 \\
20 \ldots\end{array}$ & $\begin{array}{r}6.6 \\
13.7 \\
17,0 \\
8.6 \\
8.6\end{array}$ & $\begin{array}{l}17.3 \\
54 \\
63 \\
14.7 \\
11.5\end{array}$ & $\begin{array}{r}6.6 \\
17.4 \\
17.4 \\
19.2 \\
11.9\end{array}$ & $\int_{b 30} 3.2$ & $\begin{array}{r}8.2 \\
6.4 \\
11.9 \\
5.8 \\
5.2\end{array}$ & $\begin{array}{l}59 \\
12.1 \\
7.4 \\
5.8 \\
5.0\end{array}$ & $\begin{array}{r}10.0 \\
8.8 \\
3.2 \\
2.0 \\
2.0\end{array}$ & $\begin{array}{r}7.4 \\
19.1 \\
4.6 \\
12.3 \\
5.9\end{array}$ & $\begin{array}{l}4.4 \\
4.1 \\
7.1 \\
4.1 \\
3.2\end{array}$ & $\begin{array}{r}2.8 \\
2.4 \\
35 \\
15.6 \\
5.2\end{array}$ & $\begin{array}{l}1.7 \\
1.5 \\
1.8 \\
1.8 \\
1.6\end{array}$ & $\begin{array}{l}1.9 \\
1.7 \\
2.4 \\
1.8 \\
1.4\end{array}$ \\
\hline $\begin{array}{l}21 \\
22 \\
24 \\
25\end{array}$ & $\begin{array}{l}16.1 \\
30 \\
31 \\
9.2 \\
11.6\end{array}$ & $\begin{array}{r}17.1 \\
25 \\
9.2 \\
13.1 \\
8.6\end{array}$ & $\begin{array}{r}11.9 \\
6.4 \\
5.6 \\
7.5 \\
8.9\end{array}$ & 2.8 & $\begin{array}{l}4.4 \\
4.1 \\
3.8 \\
3.6 \\
3.4\end{array}$ & $\begin{array}{l}4.4 \\
4.0 \\
3.4 \\
3.2 \\
\text { 5. } 6\end{array}$ & $\begin{array}{l}2.4 \\
3.1 \\
1.9 \\
1.6 \\
1.5\end{array}$ & $\begin{array}{l}2.9 \\
5.9 \\
5.0 \\
6.0 \\
2.8\end{array}$ & $\begin{array}{r}2.9 \\
2.9 \\
2.9 \\
7.6 \\
20\end{array}$ & $\begin{array}{l}4.0 \\
3.0 \\
2.9 \\
7.7 \\
5.0\end{array}$ & $\begin{array}{l}1.6 \\
1.8 \\
1.8 \\
1.9 \\
1.7\end{array}$ & $\begin{array}{l}1.3 \\
1.3 \\
1.4 \\
1.3 \\
1.7\end{array}$ \\
\hline $\begin{array}{l}26 \\
27 \\
30 \\
31\end{array}$ & $\begin{array}{r}8.9 \\
36 \\
11.8 \\
9.2 \\
6.9 \\
6.4\end{array}$ & $\begin{array}{l}7.6 \\
7.4 \\
7.9 \\
7.6 \\
6.0 \\
5.6\end{array}$ & $\begin{array}{l}4.8 \\
4.4 \\
8.2 \\
5.4 \\
4.2\end{array}$ & $\begin{array}{r}25 \\
5\end{array}$ & $\begin{array}{l}3.0 \\
2.9 \\
3.4 \\
2.9 \\
3.2\end{array}$ & $\begin{array}{r}3.4 \\
2.9 \\
10.7 \\
9.3 \\
3.2 \\
3.8\end{array}$ & $\begin{array}{l}1.4 \\
1.7 \\
4.6 \\
1.6 \\
1.3 \\
1.2\end{array}$ & \begin{tabular}{r}
2.6 \\
5.2 \\
10.8 \\
\hdashline-2 \\
$-2 . .$. \\
\end{tabular} & $\begin{array}{r}24 \\
5.4 \\
4.2 \\
4.0 \\
3.2 \\
5.3\end{array}$ & $\begin{array}{r}2.8 \\
2.6 \\
3.0 \\
2.7 \\
13.2\end{array}$ & $\begin{array}{l}4.6 \\
3.4 \\
1.8 \\
1.3 \\
5.3 \\
9.7\end{array}$ & $\begin{array}{r}1.3 \\
1.2 \\
1.0 \\
1.0 \\
.9\end{array}$ \\
\hline
\end{tabular}

\begin{tabular}{|c|c|c|c|c|c|c|}
\hline \multirow[b]{2}{*}{ Month } & \multicolumn{3}{|c|}{ Million gallons a day } & \multirow{2}{*}{$\begin{array}{l}\text { Second- } \\
\text { feet } \\
\text { (mean) }\end{array}$} & \multicolumn{2}{|c|}{ Total run-off } \\
\hline & Maximum & Minimum & Mean & & $\begin{array}{l}\text { Million } \\
\text { gallons }\end{array}$ & Acre-feet \\
\hline $\begin{array}{l}\text { July } \\
\text { August } \\
\text { September } \\
\text { October } \\
\text { November } \\
\text { December } \\
\text { January } \\
\text { Febriary } \\
\text { March } \\
\text { April } \\
\text { May }\end{array}$ & $\begin{array}{r}56 \\
63 \\
69 \\
145 \\
95 \\
59 \\
10.0 \\
19.1 \\
41 \\
35 \\
9.7 \\
9.4\end{array}$ & $\begin{array}{r}4.4 \\
5.6 \\
4.2 \\
2.9 \\
2.2 \\
1.2 \\
2.9 \\
2.9 \\
2.4 \\
1.3 \\
.9\end{array}$ & $\begin{array}{l}14.8 \\
16.8 \\
15.3 \\
14.4 \\
13.2 \\
8.93 \\
2.88 \\
4.61 \\
9.06 \\
6.44 \\
3.02 \\
2.49\end{array}$ & $\begin{array}{r}22.9 \\
26.0 \\
23.7 \\
22.3 \\
20.4 \\
13.8 \\
4.46 \\
7.13 \\
14.0 \\
9.96 \\
4.67 \\
3.85\end{array}$ & $\begin{array}{c}459 \\
520 \\
459 \\
447 \\
395 \\
277 \\
89.2 \\
129 \\
281 \\
193 \\
93.5 \\
74.8\end{array}$ & $\begin{array}{r}1,410 \\
1,600 \\
1,410 \\
1,370 \\
1,220 \\
850 \\
274 \\
396 \\
862 \\
593 \\
287 \\
229\end{array}$ \\
\hline The year... & 145 & .9 & 9. 36 & 14.5 & 3,420 & 10,500 \\
\hline
\end{tabular}




\section{MOANALUA STREAT FRAR HONOLULU, OAHU}

Location.- Water-stage recorder $41 / 4$ miles from mouth of stream and $51 / 4$ miles north of Honolulu post office.

Drain AGE AREA.-3.2 square miles.

Records available. - June, 1926, to June, 1929.

EXTrEmes.-Maximum discharge during year, 157 million gallons a day, or 243 second-feet July 27 (gage height, 3.17 feet); no flow for several periods during year.

1926-1929: Maximum discharge, 1,120 million gallons a day or 1,730 second-feet Mar. 16, Nov. 19, 1927 (gage height, 8.48 feet); no flow for several periods.

REMARKS.-Records poor. Water for domestic use diverted from stream 1 mile above station by 2 -inch pipe.

Discharge, in million gallons a day, 1928-29

\begin{tabular}{|c|c|c|c|c|c|c|c|c|c|c|c|c|}
\hline Day & July & Aug. & Sept. & Oet. & Nov. & Dec. & Jan. & Feb. & Mar. & Apr. & May & June \\
\hline $\begin{array}{l}1 \\
2 \\
3 \\
4 \ldots \\
5 \\
5\end{array}$ & $\begin{array}{l}0.8 \\
.55 \\
.3 \\
.09 \\
.03\end{array}$ & $\begin{array}{l}0.03 \\
.03 \\
1.7 \\
.5 \\
.06\end{array}$ & $\mathbf{0}$ & $\begin{array}{l}0 \\
0 \\
0 \\
0\end{array}$ & $\begin{array}{l}0 \\
0 \\
0 \\
1.9 \\
.3\end{array}$ & $\begin{array}{l}0 \\
0 \\
0 \\
0 \\
.35\end{array}$ & $\begin{array}{l}0 \\
0 \\
0 \\
0 \\
0\end{array}$ & $\begin{array}{l}\mathbf{0} \\
0 \\
0 \\
0 \\
0\end{array}$ & $\begin{array}{l}0.2 \\
.1 \\
.06 \\
.08 \\
.03\end{array}$ & $\begin{array}{l}0.01 \\
0 \\
0 \\
0 \\
0\end{array}$ & $\begin{array}{r}0 \\
0 \\
.0\end{array}$ & $\begin{array}{l}0 \\
0 \\
0 \\
0\end{array}$ \\
\hline 6. & $\begin{array}{r}.02 \\
.02 \\
.01 \\
.01 \\
9.2\end{array}$ & $\begin{array}{l}.03 \\
.5 \\
.04 \\
.03 \\
.04\end{array}$ & & $\begin{array}{l}0 \\
0 \\
0 \\
0 \\
0\end{array}$ & $\begin{array}{l}.25 \\
.25 \\
.2 \\
.2 \\
.25\end{array}$ & $\begin{array}{l}.1 \\
.03 \\
.02 \\
.1 \\
.8\end{array}$ & $\begin{array}{l}\mathbf{0} \\
0 \\
0 \\
0 \\
0\end{array}$ & $\begin{array}{l}0 \\
0 \\
0 \\
0 \\
0\end{array}$ & $\begin{array}{r}.03 \\
.05 \\
.03 \\
.02 \\
.01\end{array}$ & $\begin{array}{l}0 \\
0 \\
0 \\
0 \\
0\end{array}$ & & $\begin{array}{l}0 \\
0 \\
0\end{array}$ \\
\hline 11 . & $\begin{array}{l}7.1 \\
1.8 \\
1.1 \\
.9 \\
.25\end{array}$ & $\begin{array}{l}.06 \\
.5 \\
1.1 \\
.03 \\
.03\end{array}$ & $\begin{array}{l}0 \\
0\end{array}$ & $\begin{array}{l}0 \\
0 \\
0 \\
0 \\
0\end{array}$ & $\begin{array}{l}.35 \\
.25 \\
.2 \\
.2 \\
.2\end{array}$ & $\begin{array}{l}.75 \\
.9 \\
.75 \\
.55 \\
.45\end{array}$ & $\begin{array}{l}0 \\
0 \\
0 \\
0 \\
0\end{array}$ & $\begin{array}{l}0 \\
0 \\
0 \\
2.0 \\
.6\end{array}$ & .0 & $\begin{array}{l}0 \\
0 \\
0 \\
0 \\
0\end{array}$ & $\mathbf{0}$ & $\begin{array}{l}0 \\
0 \\
0 \\
0 \\
0\end{array}$ \\
\hline $\begin{array}{l}16 \\
18 \\
19\end{array}$ & $\begin{array}{l}.07 \\
3.6 \\
1.1 \\
.7 \\
.1\end{array}$ & $\begin{array}{l}.02 \\
.02 \\
2.5 \\
.8 \\
1.0\end{array}$ & $\begin{array}{l}0 \\
0 \\
0 \\
0 \\
0\end{array}$ & $\begin{array}{l}0 \\
0 \\
0 \\
9 \\
0\end{array}$ & $\begin{array}{l}.2 \\
.2 \\
.2 \\
.2 \\
.15\end{array}$ & $\begin{array}{l}.6 \\
.5 \\
.4 \\
.35 \\
.3\end{array}$ & $\begin{array}{l}0 \\
0 \\
.03 \\
.03 \\
2.3\end{array}$ & $\begin{array}{l}.4 \\
.5 \\
.35 \\
.3 \\
.3\end{array}$ & 00 & $\begin{array}{l}0 \\
0 \\
3.0 \\
.85 \\
.3\end{array}$ & $\because 0$ & $\begin{array}{l}0 \\
0 \\
0 \\
0\end{array}$ \\
\hline $\begin{array}{l}21 \\
22 \\
23 \\
24 \\
25\end{array}$ & $\begin{array}{l}.07 \\
.06 \\
.05 \\
.04 \\
.02\end{array}$ & $\begin{array}{l}.6 \\
.4 \\
.09 \\
.04 \\
.03\end{array}$ & $\begin{array}{l}0 \\
0 \\
0 \\
0 \\
0\end{array}$ & $\begin{array}{l}0 \\
0 \\
0 \\
0 \\
0\end{array}$ & $\begin{array}{l}.06 \\
.03 \\
.02 \\
.02 \\
.01\end{array}$ & $\begin{array}{l}.25 \\
6.2 \\
6.2 \\
6.15 \\
b .09\end{array}$ & $\begin{array}{l}.4 \\
.3 \\
.2 \\
.1 \\
.08\end{array}$ & $\begin{array}{l}.25 \\
.35 \\
.35 \\
.3 \\
.25\end{array}$ & & $\begin{array}{l}.07 \\
0 \\
0 \\
0\end{array}$ & $\begin{array}{l}\mathbf{0} \\
\mathbf{0} \\
0 \\
\mathbf{0} \\
\mathbf{0}\end{array}$ & $\begin{array}{l}0 \\
0 \\
0 \\
0 \\
0\end{array}$ \\
\hline $\begin{array}{l}26 \\
27 \\
28 \\
29 \\
30\end{array}$ & $\begin{array}{c}22.01 \\
5.6 \\
1.6 \\
.75 \\
.06\end{array}$ & $\begin{array}{l}.03 \\
.02 \\
.02 \\
.01\end{array}$ & $\begin{array}{l}0 \\
0 \\
0 \\
0 \\
0\end{array}$ & $\begin{array}{l}0 \\
0 \\
0 \\
0 \\
0 \\
0\end{array}$ & $\begin{array}{l}.01 \\
.01 \\
.01 \\
.01 \\
.01 \\
.--\end{array}$ & $\begin{array}{l}6.06 \\
.08 \\
.02 \\
.01 \\
.01 \\
.01\end{array}$ & \begin{tabular}{l|l}
.02 & \\
.01 & \\
.01 & \\
.02 & \\
.02 & - \\
.02 & -
\end{tabular} & $\begin{array}{l}.25 \\
.1 \\
.15 \\
-15 \\
-1\end{array}$ & $\begin{array}{r}.03 \\
.02 \\
.01 \\
.01\end{array}$ & $\begin{array}{l}0 \\
0 \\
0 \\
0 \\
0\end{array}$ & $\begin{array}{l}0 \\
1.3 \\
0.06 \\
0 \\
0\end{array}$ & $\begin{array}{l}0 \\
0 \\
0 \\
0 \\
0 \\
\end{array}$ \\
\hline \multirow{2}{*}{\multicolumn{4}{|c|}{ Month }} & \multicolumn{4}{|c|}{ Million gallons a day } & \multirow{2}{*}{\multicolumn{2}{|c|}{$\begin{array}{c}\text { Second- } \\
\text { feet } \\
\text { (mean) }\end{array}$}} & \multicolumn{3}{|c|}{ Total run-off } \\
\hline & & & & Taximum & Min & mum & Mean & & & $\begin{array}{l}\text { Million } \\
\text { gallons }\end{array}$ & Acre & feet \\
\hline $\begin{array}{l}\text { July } \\
\text { August } \\
\text { September. } \\
\text { October } \\
\text { November. } \\
\text { December... } \\
\text { January } \\
\text { February... } \\
\text { March } \\
\text { April } \\
\text { May } \\
\text { June. }\end{array}$ & 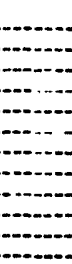 & 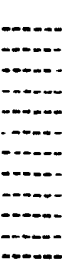 & - & $\begin{array}{c}22 \\
2.5 \\
.01 \\
.09 \\
1.9 \\
.9 \\
2.3 \\
2.0 \\
.2 \\
3.0 \\
1.3 \\
.00\end{array}$ & & $\begin{array}{l}0.01 \\
.01 \\
0 \\
0 \\
0 \\
0 \\
0 \\
0 \\
0 \\
0 \\
0 \\
0\end{array}$ & $\begin{array}{c}1.87 \\
.332 \\
.002 \\
.003 \\
.190 \\
.257 \\
.113 \\
.230 \\
.035 \\
.141 \\
.044 \\
.000\end{array}$ & & $\begin{array}{l}.89 \\
.514 \\
.003 \\
.005 \\
.294 \\
.398 \\
.175 \\
.356 \\
.054 \\
.218 \\
.068 \\
.000\end{array}$ & $\begin{array}{l}58.0 \\
10.3 \\
.05 \\
.09 \\
5.69 \\
7.98 \\
3.49 \\
6.45 \\
1.09 \\
4.24 \\
1.36 \\
.00\end{array}$ & & $\begin{array}{r}178 \\
32 \\
0 \\
0 \\
17 \\
24 \\
11 \\
20 \\
3 \\
13 \\
4 \\
0\end{array}$ \\
\hline The year. & $\ldots$ & $\ldots$ & $\ldots$ & 22 & & .00 & .270 & & .418 & 98.7 & & 302 \\
\hline
\end{tabular}




\section{KATIHI STREAM FEAR HONOLULO, OAHU}

Location. - Water-stage recorder at Kioi Pool, three-eighths mile upstream from Catholic orphanage and 5 miles north of Honolulu post office.

Drainage area. - 2.7 square miles.

RECoRds AVAILABLE. - September, 1913, to June, 1929.

Extremes.-Maximum discharge during year, 570 million gallons a day or 882 second-feet Nov. 4 (gage height, 8.60 feet); minimum uncertain, owing to missing record.

1913-1929: Maximum discharge estimated, 1,250 million gallons a day or 1,930 second-feet Jan. 16, 1921 (gage height, 14.0 feet; determined from floodmarks); minimum, 0.1 million gallons a day or 0.15 second-foot Apr. 5, 1924, May 12-25, 1926.

Remarks.-Records good. Water for domestic use diverted from stream above station.

Discharge, in million gallons a day, 1928-29

\begin{tabular}{|c|c|c|c|c|c|c|c|c|c|c|c|c|}
\hline Day & July & Aug. & Sept. & Oct. & Nov. & Dec. & Jan. & Feb. & Mar. & Apr. & May & June \\
\hline $\begin{array}{l}1 \\
2 \\
3 \\
4 \\
5 \\
5\end{array}$ & $\begin{array}{l}6.0 \\
3.7 \\
2.4 \\
2.3 \\
2.0\end{array}$ & $\begin{array}{l}3.0 \\
3.1 \\
6.0 \\
4.0 \\
3.0\end{array}$ & 2.2 & $\begin{array}{l}2.0 \\
2.5 \\
1.6 \\
1.7 \\
1.8\end{array}$ & $\begin{array}{c}1.1 \\
1.0 \\
1.0 \\
31 \\
8.4\end{array}$ & $\begin{array}{r}1.9 \\
1.9 \\
1.9 \\
1.7 \\
10.2\end{array}$ & $\begin{array}{l}2.1 \\
2.1 \\
1.8 \\
1.7 \\
1.7\end{array}$ & $\begin{array}{l}1.4 \\
1.4\end{array}$ & $\int_{66}^{3.0}$ & $\begin{array}{l}1.8 \\
1.8 \\
7.1 \\
7.4 \\
3.0\end{array}$ & & $\begin{array}{l}1.0 \\
1.8 \\
1.2 \\
1.0 \\
1.0\end{array}$ \\
\hline $\begin{array}{r}6 \\
7 \\
8 \\
9 \\
9\end{array}$ & $\begin{array}{r}1.8 \\
2.1 \\
2.0 \\
1.9 \\
27\end{array}$ & $\begin{array}{l}2.8 \\
8.7 \\
3.4 \\
2.7 \\
2.7\end{array}$ & 6 & $\begin{array}{l}1.6 \\
1.5 \\
1.5 \\
1.5 \\
1.4\end{array}$ & $\begin{array}{r}5.2 \\
10.3 \\
5.3 \\
7.0 \\
5.9\end{array}$ & $\begin{array}{l}2.5 \\
1.9 \\
1.8 \\
4.4 \\
4.8\end{array}$ & $\begin{array}{l}1.8 \\
1.6 \\
1.6 \\
1.5 \\
1.4\end{array}$ & 1.2 & 3.0 & $\begin{array}{l}2.3 \\
2.3 \\
1.9 \\
3.2 \\
2.3\end{array}$ & 1.2 & $\begin{array}{r}1.0 \\
.8 \\
.9 \\
.9 \\
1.0\end{array}$ \\
\hline 11. & $\begin{array}{l}\text { 9. } 0 \\
4.6 \\
3.2 \\
3.0 \\
2.6\end{array}$ & $\begin{array}{l}2.5 \\
5.4 \\
3.8 \\
3.7 \\
\text { 3. } 0\end{array}$ & 3.0 & $\begin{array}{l}1.5 \\
1.5 \\
1.5 \\
1.8 \\
1.3\end{array}$ & $\begin{array}{r}12.9 \\
5.6 \\
4.9 \\
5.8 \\
7.0\end{array}$ & $\begin{array}{l}5.4 \\
8.4 \\
9.5 \\
7.0 \\
4.5\end{array}$ & $\begin{array}{l}1.4 \\
1.4 \\
1.3 \\
1.4 \\
1.6\end{array}$ & & $\int_{19} 4.8$ & $\begin{array}{l}1.9 \\
1.8 \\
1.7 \\
1.8 \\
1.7\end{array}$ & & $\begin{array}{l}1.0 \\
1.0 \\
1.0 \\
1.0 \\
.9\end{array}$ \\
\hline $\begin{array}{l}16 . \ldots \\
17 \\
18 \ldots \\
20 \ldots\end{array}$ & $\begin{array}{l}2.3 \\
2.8 \\
2.3 \\
2.1 \\
1.9\end{array}$ & $\begin{array}{r}2.7 \\
2.8 \\
14.3 \\
4.9 \\
5.3\end{array}$ & 2.8 & $\begin{array}{l}1.2 \\
1.2 \\
1.3 \\
1.2 \\
1.2\end{array}$ & $\begin{array}{l}5.8 \\
4.5 \\
8.0 \\
4.8 \\
3.8\end{array}$ & $\begin{array}{l}7.8 \\
5.1 \\
3.8 \\
3.2 \\
3.0\end{array}$ & $\begin{array}{l}1.7 \\
2.6 \\
1.8 \\
1.5 \\
40\end{array}$ & 8 & 4.2 & $\begin{array}{l}1.7 \\
40 \\
8.9 \\
4.3 \\
3.1\end{array}$ & a 1.0 & $\begin{array}{l}.9 \\
1.0 \\
1.0 \\
1.0 \\
1.0\end{array}$ \\
\hline 25 & $\begin{array}{l}1.8 \\
2.0 \\
2.5 \\
1.8 \\
1.7\end{array}$ & $\begin{array}{l}\text { 4. } 0 \\
\text { 3. } \\
\text { 3. } 0 \\
\text { 3. } 6 \\
\text { 3. }\end{array}$ & $\begin{array}{l}1.6 \\
1.5 \\
1.5 \\
2.1\end{array}$ & $\begin{array}{l}1.2 \\
1.2 \\
1.2 \\
1.1 \\
1.1\end{array}$ & $\begin{array}{l}3.2 \\
3.0 \\
2.8 \\
2.6 \\
2.3\end{array}$ & $\begin{array}{l}2.6 \\
2.5 \\
2.2 \\
2.1 \\
2.3\end{array}$ & $\begin{array}{l}9.4 \\
3.8 \\
2.6 \\
2.0 \\
1.9\end{array}$ & 3.6 & 7 & 2.3 & $\begin{array}{r}1.0 \\
1.0 \\
1.0 \\
1.0 \\
.9\end{array}$ & $\begin{array}{l}.9 \\
.9 \\
.8 \\
.9\end{array}$ \\
\hline $\begin{array}{l}26 \\
28 \\
30 \\
31\end{array}$ & $\begin{array}{l}1.9 \\
29 \\
7.8 \\
3.8 \\
2.8 \\
2.5\end{array}$ & $\begin{array}{r}2.8 \\
2.6 \\
2.5 \\
2.3 \\
2.2 \\
\text { b } 2.3\end{array}$ & $\begin{array}{l}1.5 \\
1.4 \\
1.4 \\
1.4 \\
1.8\end{array}$ & \begin{tabular}{l|l}
1.0 & \\
1.0 & \\
1.1 & \\
1.4 & \\
1.3 & \\
1.1 & -
\end{tabular} & $\begin{array}{r}2.2 \\
2.1 \\
2.3 \\
2.1 \\
1.9 \\
-\end{array}$ & $\begin{array}{l}2.0 \\
1.8 \\
1.8 \\
1.8 \\
1.8 \\
1.8\end{array}$ & \begin{tabular}{l|l}
1.8 & \\
1.8 & \\
1.8 & \\
1.6 & - \\
1.6 & - \\
1.5 & -
\end{tabular} & | & $\begin{aligned} r 2.1 \\
2.1 \\
1.9 \\
2.1 \\
1.8\end{aligned}$ & 1.8 & $\begin{array}{l}1.0 \\
1.2 \\
1.0 \\
.9 \\
1.6 \\
1.9\end{array}$ & $\begin{array}{l}.8 \\
.7 \\
.6 \\
.5\end{array}$ \\
\hline \multirow{2}{*}{\multicolumn{4}{|c|}{ Montl }} & \multicolumn{4}{|c|}{ Million gallons a day } & \multirow{2}{*}{\multicolumn{2}{|c|}{$\begin{array}{l}\text { Second- } \\
\text { feet } \\
\text { (mean) }\end{array}$}} & \multicolumn{3}{|c|}{ Total run-off } \\
\hline & & & & Maximum & a Min & limum & Mean & & & $\begin{array}{l}\text { Million } \\
\text { gallons }\end{array}$ & Acr & e-feet \\
\hline $\begin{array}{l}\text { July } \\
\text { August } \\
\text { September } \\
\text { October } \\
\text { November } \\
\text { December } \\
\text { January } \\
\text { February } \\
\text { March } \\
\text { April } \\
\text { May } \\
\text { June }\end{array}$ & $\cdots$ & 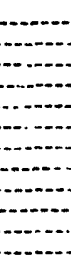 & & $\begin{array}{c}29 \\
14.3 \\
2.5 \\
31 \\
10.2 \\
40 \\
40 \\
1.8\end{array}$ & & \begin{tabular}{l}
1.7 \\
2.2 \\
1.4 \\
1.0 \\
1.0 \\
1.7 \\
1.3 \\
\hdashline 1.8 \\
.9 \\
.5
\end{tabular} & $\begin{array}{l}4.60 \\
3.85 \\
2.54 \\
1.40 \\
5.46 \\
3.66 \\
3.28 \\
3.60 \\
4.38 \\
4.02 \\
1.17 \\
.93\end{array}$ & & $\begin{array}{l}\text { 7. } 12 \\
\text { 5. } 96 \\
\text { 3.93 } \\
2.17 \\
8.45 \\
\text { 5. 66 } \\
\text { 5. } 07 \\
\text { 5. 57 } \\
6.78 \\
6.22 \\
1.81 \\
1.44\end{array}$ & $\begin{array}{l}143 \\
120 \\
76.2 \\
43.5 \\
164 \\
113 \\
102 \\
101 \\
136 \\
120 \\
36.3 \\
27.9\end{array}$ & & $\begin{array}{r}\mathbf{4 3 8} \\
\mathbf{3 6 6} \\
\mathbf{2 3 4} \\
\mathbf{1 3 3} \\
\mathbf{5 0 3} \\
\mathbf{3 4 8} \\
\mathbf{3 1 2} \\
\mathbf{3 0 9} \\
417 \\
\mathbf{3 7 0} \\
\mathbf{1 1 1} \\
\mathbf{8 6}\end{array}$ \\
\hline \multicolumn{2}{|l|}{ The jear } & $\cdots$ & & 40 & & .5 & 3.24 & & 5.01 & 1,180 & & 3,630 \\
\hline
\end{tabular}


NUUANU STREAM BELOW RESERVOIR NO. 2 WASTEWAY, NEAR HONOLULO, OAHU

Location.-Water-stage recorder on Pali road in upper Nuuanu Valley, 1 mile above end of car line and 5 miles from Honolulu post office.

Drain Age ARea.-3.4 square miles.

ReCORDS AVAILABLE.-October, 1913, to June, 1929.

Extremes.-Maximum discharge during year, 202 million gallons a day or 313 second-feet Nov. 4 (gage height, 4.02 feet); minimum, 0.85 million gallons a day or 1.3 second-feet June 29,30 .

1913-1929: Maximum discharge, 1,600 million gallons a day or 2,480 second-feet Jan. 16, 1921 (gage height, 8.74 feet; from floodmarks); minimum, 0.06 million gallons a day or 0.09 second-foot Sept. 10, 11, 1925.

REmarks.-Records fair for ordinary stages; poor for high stages and estimated periods. Reservoirs Nos. 2, 3, and 4 regulate flow, but diversion from them past station was discontinued in January. Honolulu waterworks diverts ground water from tunnels in drainage area.

Discharge, in million gallons a day, 1928-29

\begin{tabular}{|c|c|c|c|c|c|c|c|c|c|c|c|c|}
\hline IDay & July & Aug. & Sept. & \begin{tabular}{l|l} 
Oct. & $\mathrm{N}$
\end{tabular} & Nov. & Dec. & Jan. & Feb. & Mar. & Apr. & May & June \\
\hline $\begin{array}{l}2 \\
3 \ldots \\
4 \ldots \\
5\end{array}$ & $\begin{array}{l}7.6 \\
5.8 \\
3.2 \\
3.1 \\
2.8\end{array}$ & $\begin{array}{l}2.5 \\
2.8 \\
4.6 \\
3.2 \\
2.9\end{array}$ & $\begin{array}{l}2.6 \\
2.6 \\
2.4 \\
2.6 \\
2.0\end{array}$ & $\left.\begin{array}{l}1.5 \\
1.6 \\
1.5 \\
1.5 \\
1.5\end{array}\right\}$ & \begin{tabular}{c|}
1.2 \\
1.1 \\
31 \\
5.3
\end{tabular} & $\begin{array}{l}2.3 \\
2.3 \\
2.3 \\
2.2 \\
9.9\end{array}$ & $\begin{array}{l}2.4 \\
2.4 \\
2.2 \\
2.2 \\
2.1\end{array}$ & $\begin{array}{l}2.0 \\
2.0 \\
2.0 \\
1.8 \\
1.8\end{array}$ & $\begin{array}{l}2.7 \\
2.8 \\
2.8 \\
3.1 \\
5.4\end{array}$ & $\begin{array}{l}1.3 \\
1.3 \\
1.5 \\
2.2 \\
1.5\end{array}$ & $\begin{array}{l}1.8 \\
1.8 \\
1.6 \\
1.7 \\
1.5\end{array}$ & $\begin{array}{l}1.5 \\
2.2 \\
1.6 \\
1.5 \\
1.5\end{array}$ \\
\hline $\begin{array}{c}6 \ldots \\
7 \ldots \\
8 \ldots \\
9 \ldots \\
10 \ldots\end{array}$ & $\begin{array}{r}2.4 \\
2.1 \\
2.2 \\
2.0 \\
13.0\end{array}$ & $\begin{array}{l}3.1 \\
7.7 \\
3.9 \\
3.6 \\
\text { 3. } 6\end{array}$ & $\begin{array}{l}2.2 \\
2.4 \\
2.6 \\
3.2 \\
6.2\end{array}$ & $\left.\begin{array}{l}1.5 \\
1.4 \\
1.4 \\
1.3 \\
1.4\end{array}\right)$ 。 & $\begin{array}{r}4.6 \\
-5.1\end{array}$ & \begin{tabular}{l|}
3.3 \\
3.1 \\
3.2 \\
3.7 \\
3.6
\end{tabular} & $\begin{array}{l}2.0 \\
2.0 \\
1.9 \\
1.7 \\
1.7\end{array}$ & $\begin{array}{l}1.7 \\
1.7 \\
1.7 \\
1.5 \\
1.5\end{array}$ & \begin{tabular}{l|}
2.8 \\
2.8 \\
2.8 \\
3.2 \\
2.9
\end{tabular} & $\begin{array}{l}1.4 \\
1.5 \\
1.4 \\
1.5 \\
1.3\end{array}$ & $\begin{array}{l}1.5 \\
1.5 \\
1.5 \\
1.5 \\
1.4\end{array}$ & $\begin{array}{l}1.5 \\
1.5 \\
1.5 \\
1.3 \\
1.4\end{array}$ \\
\hline 5 & $\begin{array}{l}3.8 \\
2.0 \\
1.9 \\
1.8 \\
1.5\end{array}$ & $\begin{array}{l}\text { 3. } 3 \\
5.2 \\
3.9 \\
3.6 \\
3.4\end{array}$ & $\begin{array}{l}\text { 9. } 4 \\
\text { 3. } 2 \\
\text { 3. } 0 \\
4.2 \\
4.0\end{array}$ & $\begin{array}{l}1.5 \\
1.4 \\
1.4 \\
1.5 \\
1.4\end{array}$ & 4.1 & $\begin{array}{l}3.8 \\
4.5 \\
5.7 \\
5.7 \\
4.7\end{array}$ & $\begin{array}{l}1.6 \\
1.5 \\
1.4 \\
1.5 \\
1.6\end{array}$ & $\begin{array}{l}1.8 \\
1.7 \\
1.4 \\
6.0 \\
9.5\end{array}$ & $\begin{array}{l}8.2 \\
4.3 \\
4.5 \\
7.3 \\
5.7\end{array}$ & $\begin{array}{l}1.4 \\
1.3 \\
1.4 \\
1.4 \\
1.3\end{array}$ & \begin{tabular}{r|}
1.4 \\
1.4 \\
1.4 \\
1.3 \\
01.3
\end{tabular} & $\begin{array}{l}1.3 \\
1.3 \\
1.3 \\
1.3 \\
1.3\end{array}$ \\
\hline 16 & $\begin{array}{l}1.7 \\
3.1 \\
2.6 \\
2.5 \\
2.4\end{array}$ & $\begin{array}{r}3.3 \\
3.6 \\
12.6 \\
4.7 \\
4.9\end{array}$ & $\begin{array}{l}1.5 \\
1.9 \\
2.8 \\
4.9 \\
2.8\end{array}$ & $\begin{array}{l}1.4 \\
1.4 \\
1.4 \\
1.3 \\
1.2\end{array}$ & $\begin{array}{l}2.6 \\
2.2 \\
4.7 \\
2.9 \\
2.8\end{array}$ & \begin{tabular}{c|}
20 \\
6.9 \\
4.9 \\
3.9 \\
3.3
\end{tabular} & \begin{tabular}{r|}
1.8 \\
2.0 \\
2.0 \\
1.7 \\
23
\end{tabular} & $\begin{array}{r}7.9 \\
9.4 \\
3.4 \\
10.3 \\
5.2\end{array}$ & $\begin{array}{l}4.6 \\
3.9 \\
3.6 \\
2.9 \\
2.7\end{array}$ & $\begin{array}{r}1.3 \\
13.5 \\
2.8 \\
1.9 \\
1.8\end{array}$ & $\begin{array}{r}1.3 \\
\cdot 1.3\end{array}$ & $\begin{array}{l}1.3 \\
1.3 \\
1.3 \\
1.2 \\
1.2\end{array}$ \\
\hline 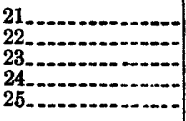 & $\begin{array}{l}2.4 \\
2.6 \\
3.0 \\
2.4 \\
2.4\end{array}$ & \begin{tabular}{l|l}
4.2 \\
3.9 \\
3.6 \\
3.7 \\
3.3
\end{tabular} & $\begin{array}{l}3.4 \\
2.8 \\
2.8 \\
2.6 \\
2.6\end{array}$ & $\begin{array}{l}1.2 \\
1.2 \\
1.2 \\
1.1 \\
1.1\end{array}$ & $\begin{array}{l}2.7 \\
2.7 \\
2.6 \\
2.5 \\
2.4\end{array}$ & $\begin{array}{l}\text { 2. } 9 \\
3.0 \\
\text { 3. } \\
\text { 3. } \\
\text { 3. } 0\end{array}$ & \begin{tabular}{r|}
18.0 \\
15.2 \\
12.6 \\
10.5 \\
7.6
\end{tabular} & $\begin{array}{l}3.6 \\
3.3 \\
3.7 \\
2.9 \\
2.6\end{array}$ & $\begin{array}{l}2.5 \\
2.3 \\
3.7 \\
5.7 \\
6.1\end{array}$ & $\begin{array}{l}1.8 \\
1.8 \\
1.9 \\
1.8 \\
1.7\end{array}$ & $\begin{array}{l}1.3 \\
1.2 \\
1.3 \\
1.3 \\
1.2\end{array}$ & $\begin{array}{l}1.2 \\
1.2 \\
1.1 \\
1.1 \\
1.1\end{array}$ \\
\hline $\begin{array}{l}26 \\
27 \\
28 \\
30 \\
31\end{array}$ & $\begin{array}{r}2.4 \\
31 \\
16.0 \\
3.8 \\
2.8 \\
2.2\end{array}$ & $\begin{array}{l}3.1 \\
2.8 \\
2.6 \\
2.4 \\
2.4 \\
2.5\end{array}$ & $\begin{array}{l}2.5 \\
2.0 \\
1.6 \\
1.6 \\
1.6\end{array}$ & $\begin{array}{l}1.1 \\
1.1 \\
1.1 \\
1.4 \\
1.4 \\
1.3\end{array}$ & $\begin{array}{l}2.4 \\
2.4 \\
2.6 \\
2.6 \\
2.4\end{array}$ & $\begin{array}{l}2.8 \\
2.8 \\
3.2 \\
2.9 \\
2.4 \\
2.3\end{array}$ & $\begin{array}{l}3.5 \\
3.0 \\
\text { 3. } \\
2.4 \\
2.3 \\
2.2\end{array}$ & $\begin{array}{r}\mathbf{2 . 6} \\
\mathbf{5 . 0} \\
\mathbf{3 . 1} \\
\hdashline-. \\
\\
\hdashline-\end{array}$ & $\begin{array}{l}4.4 \\
1.3 \\
1.3 \\
1.2 \\
1.4 \\
1.3\end{array}$ & $\begin{array}{l}1.7 \\
1.7 \\
1.8 \\
1.9 \\
1.8\end{array}$ & $\begin{array}{l}1.3 \\
1.7 \\
1.4 \\
1.4 \\
1.5 \\
2.5\end{array}$ & $\begin{array}{l}1.1 \\
1.0 \\
1.0 \\
.9 \\
.9\end{array}$ \\
\hline \multirow{2}{*}{\multicolumn{4}{|c|}{ Month }} & \multicolumn{4}{|c|}{ Million gallons a day } & \multirow{2}{*}{\multicolumn{2}{|c|}{$\begin{array}{l}\text { Second- } \\
\text { feet } \\
\text { (mean) }\end{array}$}} & \multicolumn{3}{|c|}{ Total run-off } \\
\hline & & & & Maximum & Min & aimum & Mear & & & $\begin{array}{l}\text { Million } \\
\text { gallons }\end{array}$ & Acre & e-feet \\
\hline \multicolumn{4}{|c|}{ 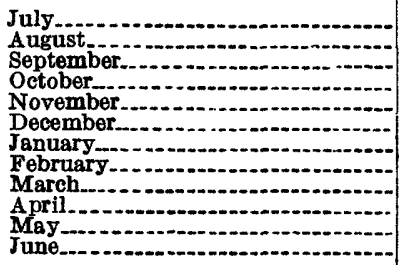 } & \begin{tabular}{c|c|}
31 \\
12.6 \\
9.4 \\
1.6 \\
31 \\
20 \\
23 \\
10.3 \\
8.2 \\
13.5 \\
2.5 \\
2.2
\end{tabular} & & $\begin{array}{l}1.5 \\
2.4 \\
1.5 \\
1.1 \\
-2.2 \\
1.4 \\
1.4 \\
1.2 \\
1.3 \\
1.2\end{array}$ & $\begin{array}{l}4 . \\
3 . \\
3 . \\
1 . \\
4 . \\
4 . \\
4 . \\
3 . \\
3 . \\
2 . \\
1 . \\
1 .\end{array}$ & $\begin{array}{l}47 \\
90 \\
00 \\
35 \\
09 \\
21 \\
48 \\
61 \\
55\end{array}$ & $\begin{array}{l}6.92 \\
6.03 \\
4.64 \\
2.09 \\
6.33 \\
6.51 \\
6.93 \\
5.59 \\
5.49 \\
3.14 \\
2.26 \\
2.01\end{array}$ & $\begin{array}{l}138 \\
121 \\
90.0 \\
41.7 \\
123 \\
131 \\
139 \\
101 \\
110 \\
60.9 \\
45.2 \\
38.9\end{array}$ & & $\begin{array}{l}\mathbf{4 2 5} \\
371 \\
276 \\
128 \\
377 \\
401 \\
\mathbf{4 2 6} \\
310 \\
338 \\
187 \\
139 \\
120\end{array}$ \\
\hline \multicolumn{4}{|c|}{ The year. } & 31 & & .9 & 3. & 12 & 4.83 & 1,140 & & 3,500 \\
\hline
\end{tabular}

- Partly estimated. 


\section{WEST BEANCH OF MANOA STREAM TEAR HONOLOLO, OAHO}

LocATION-Water-stage recorder 75 feet above lower highway bridge and 4 miles northeast of Honolulu post office.

Drain age are A. - 1.1 square miles.

Records AVAIt ABLE.- May, 1913, to January, 1921; August, 1925, to June, 1929.

Exтremes.-Maximum discharge during year, 550 million gallons a day or 851 second-feet Jan. 20 (gage height, 3.45 feet); minimum, 0.15 million gallons a day or 0.25 second-foot Nov. 2 .

1913-1921, 1926-1929: Maximum stage, 10.4 feet Jan. 16, 1921, from floodmarks (discharge (estimated), 2,100 million gallons a day or 3,250 second-feet); minimum, about 0.05 million gallons a day or 0.08 secondfoot Mar. 16, 22, 1926.

REMarks. - Records fair for low stages; good for medium stages; poor for high stages and estimated period. Gage datum raised 3.40 feet Mar. 16, 1928. No diversions above station.

Discharge, in million gallons a day, 1928-29

\begin{tabular}{|c|c|c|c|c|c|c|c|c|c|c|c|c|}
\hline Day & July & Aug. & Sept. & Oct. $N$ & Nov. & Dec. & Jan. & Feb. & Mar. & Apr. & May & June \\
\hline $\begin{array}{l}2 \ldots \\
3 \\
4 \ldots \\
5 \\
5\end{array}$ & $\begin{array}{l}4.9 \\
3.1 \\
2.0 \\
1.6 \\
1.3\end{array}$ & $\begin{array}{l}1.5 \\
2.2 \\
4.9 \\
2.4 \\
2.2\end{array}$ & $\begin{array}{l}1.6 \\
1.5 \\
1.6 \\
2.4 \\
1.3\end{array}$ & \begin{tabular}{l|}
0.7 \\
.8 \\
.7 \\
.8 \\
1.0
\end{tabular} & $\begin{array}{c}0.3 \\
.25 \\
.25 \\
33.6\end{array}$ & $\begin{array}{r}0.6 \\
.6 \\
1.1 \\
.9 \\
5.7\end{array}$ & $\begin{array}{r}1.5 \\
1.1 \\
.9 \\
.7 \\
.7\end{array}$ & $\begin{array}{l}0.6 \\
.6 \\
.5 \\
.5 \\
.4\end{array}$ & $\begin{array}{l}1.6 \\
2.6 \\
1.8 \\
2.0 \\
3.2\end{array}$ & $\begin{array}{l}1.1 \\
1.0 \\
.9 \\
2.5 \\
5.5\end{array}$ & $\begin{array}{r}0.9 \\
1.0 \\
.9 \\
.6 \\
2.4\end{array}$ & $\begin{array}{l}0.8 \\
1.1 \\
.8 \\
.8 \\
.7\end{array}$ \\
\hline 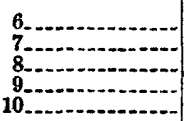 & \begin{tabular}{r|}
1.2 \\
4.1 \\
4.0 \\
1.2 \\
33
\end{tabular} & $\begin{array}{l}2.0 \\
2.8 \\
1.6 \\
1.5 \\
5.5\end{array}$ & $\begin{array}{r}1.3 \\
1.5 \\
1.8 \\
2.0 \\
13.5\end{array}$ & $\begin{array}{l}.6 \\
.55 \\
.55 \\
.6 \\
.6\end{array}$ & $\begin{array}{l}4.9 \\
7.8 \\
6.7 \\
5.3 \\
3.3\end{array}$ & $\begin{array}{r}1.0 \\
.8 \\
.8 \\
3.8 \\
2.8\end{array}$ & $\begin{array}{l}.8 \\
.7 \\
.6 \\
.55 \\
.55\end{array}$ & $\begin{array}{l}.55 \\
.4 \\
.4 \\
.4 \\
.25\end{array}$ & $\begin{array}{r}2.4 \\
1.8 \\
a 2.6 \\
1.8 \\
2.8\end{array}$ & $\begin{array}{l}1.5 \\
1.2 \\
1.2 \\
1.0 \\
1.1\end{array}$ & $\begin{array}{l}.7 \\
.6 \\
.55 \\
.5 \\
.6\end{array}$ & $\begin{array}{l}.55 \\
.6 \\
.9 \\
.55 \\
.6\end{array}$ \\
\hline (1) & \begin{tabular}{r|}
10.0 \\
3.4 \\
2.4 \\
2.4 \\
1.6
\end{tabular} & $\begin{array}{l}2.2 \\
5.3 \\
3.1 \\
3.4 \\
2.4\end{array}$ & $\begin{array}{r}15.0 \\
1.8 \\
1.3 \\
1.1 \\
1.0\end{array}$ & $\begin{array}{l}.5 \\
.5 \\
.55 \\
.5 \\
.7\end{array}$ & $\begin{array}{l}9.4 \\
2.4 \\
3.1 \\
3.7 \\
4.0\end{array}$ & $\begin{array}{l}2.4 \\
3.9 \\
2.6 \\
4.1 \\
2.8\end{array}$ & $\begin{array}{l}.5 \\
.7 \\
.6 \\
.6 \\
.6\end{array}$ & $\begin{array}{r}.5 \\
.7 \\
.4 \\
3.0 \\
3.9\end{array}$ & $\begin{array}{r}2.5 \\
20 \\
4.9 \\
5.7 \\
\times 3.1\end{array}$ & $\begin{array}{l}.9 \\
.8 \\
.7 \\
.55 \\
.7\end{array}$ & $\begin{array}{l}.55 \\
.5 \\
.4 \\
.5 \\
.5\end{array}$ & $\begin{array}{l}.6 \\
.55 \\
.8 \\
.6 \\
.55\end{array}$ \\
\hline $\begin{array}{l}16 \\
17 \\
19\end{array}$ & $\begin{array}{l}2.0 \\
4.4 \\
2.0 \\
1.6 \\
1.6\end{array}$ & $\begin{array}{r}2.0 \\
10.1 \\
16.2 \\
3.1 \\
4.9\end{array}$ & $\begin{array}{l}1.0 \\
1.2 \\
\text { 3.1 } \\
\text { 1. } \\
1.6\end{array}$ & \begin{tabular}{l|l}
.6 & \\
.55 & \\
.7 & 1 \\
.8 & \\
0.7 &
\end{tabular} & $\begin{array}{r}2.4 \\
2.2 \\
10.4 \\
2.6 \\
2.0\end{array}$ & \begin{tabular}{l|}
36 \\
8.1 \\
3.4 \\
2.4 \\
1.6
\end{tabular} & $\begin{array}{c}1.3 \\
2.0 \\
1.4 \\
1.1 \\
34\end{array}$ & $\begin{array}{l}6.3 \\
15.2 \\
3.4 \\
7.3 \\
5.6\end{array}$ & 1.6 & $\begin{array}{c}.55 \\
27^{.55} \\
4.4 \\
1.6\end{array}$ & $\begin{array}{l}.5 \\
.5 \\
.5 \\
.55 \\
.55\end{array}$ & $\begin{array}{l}.7 \\
.3 \\
.9 \\
.55 \\
.5\end{array}$ \\
\hline 221 & $\begin{array}{l}1.6 \\
2.5 \\
2.2 \\
1.5 \\
1.3\end{array}$ & $\begin{array}{l}3.1 \\
3.4 \\
2.2 \\
4.6 \\
2.4\end{array}$ & $\begin{array}{r}1.7 \\
1.0 \\
.8 \\
.9 \\
1.6\end{array}$ & $\begin{array}{l}.6 \\
.7 \\
.6 \\
.5 \\
.4\end{array}$ & $\begin{array}{l}1.6 \\
1.3 \\
1.3 \\
1.2 \\
1.3\end{array}$ & \begin{tabular}{l|}
1.6 \\
1.6 \\
1.5 \\
1.3 \\
1.1
\end{tabular} & $\begin{array}{l}5.8 \\
2.4 \\
1.5 \\
1.5 \\
1.2\end{array}$ & $\begin{array}{l}2.6 \\
2.6 \\
3.4 \\
2.2 \\
1.8\end{array}$ & $\begin{array}{r}1.0 \\
1.1 \\
2.0 \\
2.4\end{array}$ & $\begin{array}{l}1.2 \\
1.1 \\
.9 \\
1.1 \\
1.1\end{array}$ & $\begin{array}{l}.5 \\
.5 \\
.6 \\
.6 \\
.6\end{array}$ & $\begin{array}{l}.5 \\
.55 \\
.5 \\
.8 \\
.55\end{array}$ \\
\hline $\begin{array}{l}26 \\
27 \\
28 \\
29 \\
31 \\
31\end{array}$ & $\begin{array}{r}1.6 \\
18.0 \\
4.0 \\
2.0 \\
1.6 \\
1.3\end{array}$ & $\begin{array}{l}2.0 \\
1.8 \\
1.5 \\
1.5 \\
1.3 \\
1.2\end{array}$ & $\begin{array}{l}.9 \\
.8 \\
.7 \\
.6 \\
.8\end{array}$ & $\begin{array}{l}.4 \\
.4 \\
.7 \\
.55 \\
.55 \\
.4\end{array}$ & $\begin{array}{l}.1 \\
.9 \\
.9 \\
.8 \\
.8\end{array}$ & $\begin{array}{l}1.1 \\
1.0 \\
1.0 \\
1.0 \\
1.0 \\
.9\end{array}$ & $\begin{array}{l}1.1 \\
1.0 \\
.9 \\
.8 \\
.7 \\
.55\end{array}$ & $\begin{array}{l}1.5 \\
2.5 \\
2.8\end{array}$ & $\begin{array}{l}2.2 \\
1.3 \\
1.2 \\
1.0 \\
.9 \\
1.4\end{array}$ & $\begin{array}{r}1.0 \\
.9 \\
.8 \\
.7 \\
2.1 \\
\end{array}$ & $\begin{array}{r}.9 \\
4.2 \\
1.0 \\
.8 \\
.8 \\
1.4\end{array}$ & $\begin{array}{l}.55 \\
.5 \\
.4 \\
.4 \\
.35\end{array}$ \\
\hline \multirow{2}{*}{\multicolumn{4}{|c|}{ Month }} & \multicolumn{4}{|c|}{ Million gallons a day } & \multirow{2}{*}{\multicolumn{2}{|c|}{$\begin{array}{c}\text { Second- } \\
\text { feet } \\
\text { (mean) }\end{array}$}} & \multicolumn{3}{|c|}{ Total run-off } \\
\hline & & & & Maximum & Min & imum & Mean & & & $\begin{array}{l}\text { Million } \\
\text { gallons }\end{array}$ & Acr & e-feet \\
\hline \multicolumn{4}{|c|}{$\begin{array}{l}\text { July } \\
\text { August } \\
\text { September } \\
\text { October } \\
\text { November } \\
\text { December } \\
\text { January } \\
\text { February } \\
\text { March } \\
\text { Mpril } \\
\text { May } \\
\text { Mune }\end{array}$} & $\begin{array}{l}33 \\
16.2 \\
15.0 \\
1.0 \\
33 \\
36 \\
34 \\
15.2 \\
20 \\
27 \\
4.2 \\
1.1\end{array}$ & & $\begin{array}{l}1.2 \\
1.2 \\
.6 \\
.4 \\
.25 \\
.6 \\
.5 \\
.25 \\
.9 \\
.55 \\
.4 \\
.3\end{array}$ & $\begin{array}{l}4.05 \\
3.36 \\
2.32 \\
.605 \\
4.02 \\
3.18 \\
2.20 \\
2.51 \\
2.67 \\
2.19 \\
.829 \\
.618\end{array}$ & & $\begin{array}{l}6.27 \\
5.20 \\
.59 \\
.936 \\
6.22 \\
4.92 \\
3.40 \\
3.88 \\
4.13 \\
3.39 \\
1.28 \\
.956\end{array}$ & $\begin{array}{c}125 \\
104 \\
69.7 \\
18.8 \\
121 \\
98.7 \\
68.4 \\
70.3 \\
82.9 \\
65.6 \\
25.7 \\
18.6\end{array}$ & & $\begin{array}{r}385 \\
320 \\
214 \\
58 \\
370 \\
303 \\
209 \\
216 \\
254 \\
202 \\
79 \\
\mathbf{5 7}\end{array}$ \\
\hline \multicolumn{4}{|c|}{ The year. } & 36 & & .25 & 2.38 & & 3.68 & 869 & & 2,670 \\
\hline
\end{tabular}

- Partly estimated. 


\section{EAST BRANCH OF MANOA STREAM NEAR HONOLULU, OAHU}

Location.-Water-stage recorder just below highway bridge, 400 feet upstream from confluence with West Branch of Manoa Stream, and 4 miles northeast of Honolulu post office.

Drainage area. - 1.0 square mile.

Records available.-May, 1913, to January, 1921; August, 1925, to June, 1929.

Extremes.-Maximum discharge during year, 129 million gallons a day or 200 second-feet Nov. 4 (gage height, 2.40 feet); minimum, 0.75 million gallons a day or 1.16 second-feet May 20-22.

1913-1921 1926-1929: Maximum gage height (determined from floodmarks), 10.4 feet Jan. 16, 1921 (discharge (estimated), 2,000 million gallons a day or 3,090 second-feet); minimum, 0.4 million gallons a day or 0.6 secondfoot June $7,8,1926$.

REMARKs.- Records fair for ordinary stages; good for medium stages; poor for high stages and estimated periods. Water is diverted from stream above station by East Manoa ditch and from tunnels in drainage area by Honolulu water works. Gage datum raised 3.40 feet Mar. 19, 1928 .

Discharge, in million gallons a day, 1928-29

\begin{tabular}{|c|c|c|c|c|c|c|c|c|c|c|c|c|}
\hline Day & July & Aug. & Sept. & Oct. & Nov. & Dec. & Jan. & Feb. & Mar. & Apr. & May & June \\
\hline & $\begin{array}{l}5.8 \\
4.8 \\
3.7 \\
3.4 \\
3.1\end{array}$ & $\begin{array}{l}2.0 \\
3.1 \\
4.1 \\
2.5 \\
2.0\end{array}$ & $\begin{array}{l}2.2 \\
2.0 \\
2.2 \\
2.5 \\
2.0\end{array}$ & $\begin{array}{r}2.0 \\
2.0 \\
1.8 \\
-2.0 \\
-2.0 \\
2.0\end{array}$ & $\begin{array}{r}1.5 \\
1.5 \\
1.5 \\
12.6 \\
6.6\end{array}$ & $\begin{array}{r}a 1.8 \\
1.8 \\
2.8 \\
1.8 \\
6.6\end{array}$ & $\begin{array}{l}2.8 \\
2.5 \\
1.8 \\
1.8 \\
1.6\end{array}$ & $\begin{array}{l}1.1 \\
1.1 \\
1.1 \\
1.1 \\
1.1\end{array}$ & $\begin{array}{r}2.0 \\
2.8 \\
-2.0\end{array}$ & $\begin{array}{l}1.4 \\
1.4 \\
1,4 \\
3.6 \\
5.7\end{array}$ & $\begin{array}{l}1.4 \\
1.5 \\
1.4 \\
1.4 \\
1.8\end{array}$ & $\begin{array}{l}1.1 \\
1.2 \\
1.0 \\
1.1 \\
1.1\end{array}$ \\
\hline & $\begin{array}{r}2.8 \\
4.1 \\
4.4 \\
3.1 \\
11.5\end{array}$ & $\begin{array}{l}2.0 \\
3.7 \\
2.0 \\
1.8 \\
4.1\end{array}$ & $\begin{array}{l}2.0 \\
2.2 \\
2.2 \\
2.0 \\
6.0\end{array}$ & 2.0 & $\begin{array}{l}5.1 \\
7.0 \\
6.5 \\
5.8 \\
4.1\end{array}$ & $\begin{array}{l}2.0 \\
1.8 \\
2.0 \\
4.4 \\
3.7\end{array}$ & $\begin{array}{l}1.6 \\
1.6 \\
1.6 \\
1.6 \\
1.6\end{array}$ & $\begin{array}{l}1.1 \\
1.0 \\
1.0 \\
1.0 \\
1.0\end{array}$ & $\begin{array}{l}2.8 \\
4.1\end{array}$ & $\begin{array}{l}2.5 \\
2.0 \\
2.2 \\
2.0 \\
2.0\end{array}$ & $\begin{array}{l}1.6 \\
1.5 \\
1.5 \\
1.5 \\
1.5\end{array}$ & $\begin{array}{l}.8 \\
1.0 \\
1.4 \\
1.8\end{array}$ \\
\hline 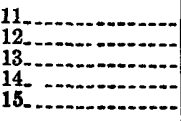 & $\begin{array}{l}7.0 \\
3.7 \\
3.4 \\
3.7 \\
3.1\end{array}$ & $\begin{array}{l}2.5 \\
4.8 \\
3.4 \\
4.1 \\
3.1\end{array}$ & $\begin{array}{l}4.1 \\
2.5 \\
2.2 \\
2.0 \\
2.0\end{array}$ & $\begin{array}{r} \\
2.0 \\
2.0 \\
2.0 \\
2.0\end{array}$ & $\begin{array}{l}6.6 \\
3.1 \\
4.8 \\
4.8 \\
4.4\end{array}$ & $\begin{array}{l}3.1 \\
3.7 \\
3.1 \\
3.4 \\
3.7\end{array}$ & $\begin{array}{l}1.6 \\
1.6 \\
1.6 \\
1.5 \\
2.0\end{array}$ & $\begin{array}{l}1.1 \\
1.2 \\
1.1 \\
2.8 \\
2.4\end{array}$ & $\begin{array}{l}3.4 \\
9.2 \\
4.8 \\
4.9 \\
4.1\end{array}$ & $\begin{array}{l}2.0 \\
1.8 \\
1.8 \\
1.6 \\
1.8\end{array}$ & $\begin{array}{l}1.5 \\
1.4 \\
1.4 \\
1.5 \\
1.2\end{array}$ & $\begin{array}{l}1.1 \\
1.1 \\
1.0 \\
1.0 \\
1.1\end{array}$ \\
\hline - & $\begin{array}{l}3.7 \\
5.1 \\
3.7 \\
3.1 \\
2.8\end{array}$ & $\begin{array}{l}3.1 \\
6.3 \\
9.8 \\
4.1 \\
6.1\end{array}$ & $\begin{array}{l}2.2 \\
2.5 \\
2.8 \\
3.4 \\
2.2\end{array}$ & $\begin{array}{r}2.0 \\
2.0 \\
2.0 \\
2.0 \\
-1.8\end{array}$ & $\begin{array}{l}3.4 \\
3.4 \\
7.0 \\
3.7 \\
3.7\end{array}$ & $\begin{array}{r}14.7 \\
5.5 \\
3.4 \\
3.1 \\
2.8\end{array}$ & \begin{tabular}{r|}
2.5 \\
3.4 \\
2.5 \\
2.0 \\
12.8
\end{tabular} & $\begin{array}{l}4.1 \\
6.6 \\
2.0 \\
7.0 \\
3.1\end{array}$ & $\begin{array}{l}3.4 \\
2.8 \\
2.5 \\
2.5 \\
2.2\end{array}$ & $\begin{array}{r}1.8 \\
1.8 \\
14.8 \\
4.4 \\
2.5\end{array}$ & $\begin{array}{r}.9 \\
.9 \\
.8 \\
1.0 \\
.8\end{array}$ & $\begin{array}{l}1 . \\
1 . \\
1 . \\
1 .\end{array}$ \\
\hline 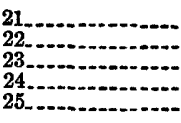 & $\begin{array}{l}2.8 \\
3.4 \\
3.4 \\
2.2 \\
2.0\end{array}$ & $\begin{array}{l}3.4 \\
3.4 \\
2.8 \\
5.1 \\
2.5\end{array}$ & $\begin{array}{l}2.0 \\
1.9 \\
1.5 \\
1.5 \\
4.0\end{array}$ & $\begin{array}{l}1.8 \\
1.8 \\
1.8 \\
1.8 \\
1.6\end{array}$ & $\begin{array}{l}3.1 \\
2.5 \\
2.2 \\
2.0 \\
2.0\end{array}$ & $\begin{array}{l}2.2 \\
2.2 \\
2.2 \\
2.2 \\
2.2\end{array}$ & $\begin{array}{l}5.1 \\
2.8 \\
2.0 \\
2.0 \\
1.8\end{array}$ & $\begin{array}{r}2.0 \\
-5.1\end{array}$ & $\begin{array}{l}2.0 \\
1.5 \\
1.4 \\
1.8 \\
2.5\end{array}$ & $\begin{array}{l}2.0 \\
1.6 \\
1.5 \\
1.5 \\
1.5\end{array}$ & $\begin{array}{l}.8 \\
.8 \\
.9 \\
.9 \\
.8\end{array}$ & 1. \\
\hline (2) & $\begin{array}{l}2.0 \\
9.1 \\
4.4 \\
2.5 \\
2.0 \\
2.0\end{array}$ & $\begin{array}{l}3.1 \\
2.5 \\
2.2 \\
2.5 \\
2.5 \\
2.5\end{array}$ & $\begin{array}{l}1.6 \\
1.6 \\
1.8 \\
1.8 \\
2.5\end{array}$ & \begin{tabular}{l|l}
1.6 & \\
1.6 & \\
1.5 & \\
1.5 & \\
1.5 & \\
1.5 &..
\end{tabular} & $\begin{array}{r}1.8 \\
1.8 \\
1.8 \\
1.8 \\
.1 .8 \\
\cdots \cdots . . .\end{array}$ & $\begin{array}{l}2.5 \\
2.5 \\
2.5 \\
2.2 \\
2.0 \\
2.2\end{array}$ & \begin{tabular}{l|l}
1.8 & \\
1.8 & \\
1.8 & \\
1.8 & - \\
1.5 & -
\end{tabular} & & $\begin{array}{l}2.0 \\
1.5 \\
1.5 \\
1.1 \\
1.2 \\
1.5\end{array}$ & $\begin{array}{l}1.5 \\
1.4 \\
1.5 \\
1.5 \\
1.6\end{array}$ & $\begin{array}{l}1.1 \\
1.8 \\
1.2 \\
1.1 \\
1.2 \\
1.4\end{array}$ & 1. \\
\hline \multirow{2}{*}{\multicolumn{4}{|c|}{ Month }} & \multicolumn{4}{|c|}{ Million gallons a day } & \multirow{2}{*}{\multicolumn{2}{|c|}{$\begin{array}{l}\text { Second- } \\
\text { feet } \\
\text { (mean) }\end{array}$}} & \multicolumn{3}{|c|}{ Total run-of } \\
\hline & & & & Maximum & \multicolumn{2}{|c|}{ m Minimum } & Mean & & & $\begin{array}{l}\text { Million } \\
\text { gallons }\end{array}$ & \multicolumn{2}{|c|}{ Acre-feet } \\
\hline \multicolumn{4}{|c|}{$\begin{array}{l}\text { July } \\
\text { August } \\
\text { September- } \\
\text { October- } \\
\text { November } \\
\text { Docember- } \\
\text { January } \\
\text { February } \\
\text { March } \\
\text { April }\end{array}$} & $\begin{aligned} 11.5 \\
9.8 \\
6.0 \\
2.0 \\
12.6 \\
14.7 \\
12.8 \\
7.0 \\
9.2 \\
14.8 \\
1.8 \\
1.4\end{aligned}$ & & $\begin{array}{l}2.0 \\
1.8 \\
1.5 \\
1.5 \\
1.5 \\
1.8 \\
1.5 \\
1.1 \\
1.4 \\
.8\end{array}$ & $\begin{array}{l}3 . \\
3 . \\
2 . \\
1 . \\
3 . \\
2 . \\
2 .\end{array}$ & & $\begin{array}{l}\text { 6. } 08 \\
5.34 \\
3.68 \\
2.88 \\
6.08 \\
5.00 \\
3.70 \\
3.64 \\
4.27 \\
3.82 \\
1.92 \\
1.61\end{array}$ & $\begin{array}{c}122 \\
107 \\
71.4 \\
57.6 \\
118 \\
100 \\
74.0 \\
65.9 \\
85.5 \\
74.1 \\
38.5 \\
31.1\end{array}$ & & $\begin{array}{l}37 \\
32 \\
21\end{array}$ \\
\hline \multicolumn{4}{|c|}{ The year } & 14.8 & & .8 & 2.59 & & 4.01 & 495 & & 2,900 \\
\hline
\end{tabular}

a Partly estimated. 
EAST MAFOA DITCH TEAR HOROLULU, OAEU

Location.-Water-stage recorder 150 feet east of lower highway and gaging station on East Branch of Manoa Stream and 4 miles northeast of Honolulu post office.

Records avallable.-May, 1915, to December, 1916; January, 1918, to January, 1921; August, 1925, to June, 1929.

EXTREMms.-Maximum discharge during year, 6.4 million gallons a day or 9.9 second-feet Nov. 4 (gage height, 1.39 feet); minimum, 0.05 million gallons a day or 0.08 second-foot Aug. 19 .

1915-1917, 1918-1921, 1926-1929: Maximum discharge, about 26 million gallons a day or 40 second-feet Jan. 16, 1921 (gage height, 2.27 feet); no flow Aug. 26, 1927.

RmMarKs.- Records fair. Water diverted from East Manoa Stream about onefourth mile above station by means of crude-stone dam.

Discharge, in million gallons a day, 1988-29

\begin{tabular}{r|c|c|c|c|c|c|c|c|c|c|c|c}
\hline Day & July & Aug. & Sept. & Oct. & Nov. & Dec. & Jan. & Feb. & Mar. & Apr. & May & June \\
\hline & & & & & & & & & & \\
\hline 1
\end{tabular}

a Partly estimated. 
PUKELE STREAM NEAR HONOLULU, OAHU

Location.- Water-stage recorder 200 feet upstream from Palolo belt-road bridge, five-eighths mile above confluence of Pukele and Waiomao Streams, and 4\%, miles east of Honolulu post office.

Drain age AREa. - 1.2 square miles.

Records available.-April, 1912, to September, 1913; June, 1926, to June, 1929.

ExTREMEs.-Maximum discharge during year, 104 million gallons a day or 161 second-feet Jan. 20 (gage height, 3.18 feet; from floodmarks); minimum uncertain, owing to plugged intake.

1912-13, 1926-1929: Maximum discharge, 360 million gallons a day or 557 second-feet May 16, 1927 (gage height, 6.13 feet); minimum, 0.15 million gallons a day, or 0.25 second-foot June $3,1926$.

REMARKs. - Records fair for ordinary stages; poor for high stages and estimated periods. 2-inch pipe diverts water from stream above station.

Discharge, in million gallons a day, 1928-29

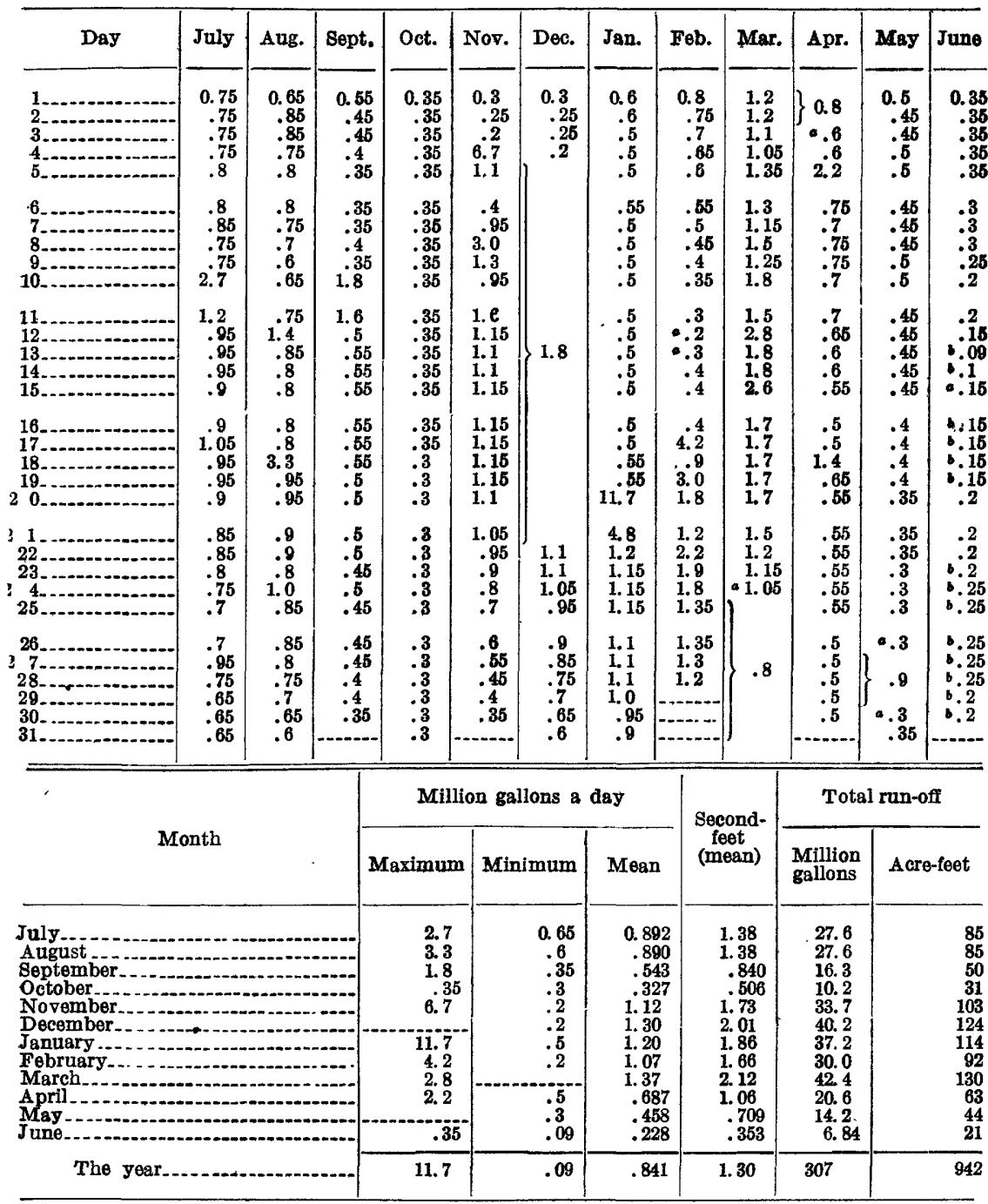

a Partly estimated.

- Estimated. 
WAIOMAO STREAM ABOVE PUKBLE STREAM, NEAR HONOLULU, OAHU

Locstion.-Water-stage recorder 300 feet west of road, 1 mile upstream from confluence of $W$ aiomao and Pukele Streams, and 5 miles east of Honolulu post office.

Drainage area.- -1.0 square mile.

Records available.-April, 1911, to December, 1912; June, 1926, to June, 1929.

EXTREMEs.-Maximum discharge during year, 33 million gallons a day or 51 second-feet Jan. 20 (gage height, 1.58 feet); minimum, 0.02 million gallons a day or 0.03 second-feet Nov. 3,4 :

1911-12, 1926-1929: Maximum discharge at least 440 million gallons a day or 681 second-feet May 16, 1927 (gage height, at least 5.52 feet; from: floodmarks); no flow July 27, to Aug. 5, 1926.

REmarks. - Records fair for ordinary stages; poor for high stages and estimated periods. No diversions from stream above station.

Discharge, in million gallons a day, 1928-29

\begin{tabular}{|c|c|c|c|c|c|c|c|c|c|c|c|c|}
\hline Day & July & Ang. & Sept. & Oct. I & Nov. & Dec. & Jan. & Feb. & Mar. & Apr. & May & June \\
\hline $\begin{array}{l}2 \ldots \\
3 \\
4 \\
5\end{array}$ & $\begin{array}{c}1.4 \\
1.0 \\
.65 \\
.7 \\
.55\end{array}$ & $\begin{array}{c}.8 \\
1.0 \\
.7 \\
.55\end{array}$ & $\begin{array}{l}0.35 \\
.25 \\
.3 \\
.35 \\
.25\end{array}$ & $\begin{array}{l}0.3 \\
.25 \\
.2 \\
.2 \\
.15\end{array}$ & $\begin{array}{c}0.05 \\
.1 \\
.05 \\
2.5\end{array}$ & 2.0 & 0.5 & $\begin{array}{l}0.09 \\
.08 \\
.08 \\
.08\end{array}$ & $\begin{array}{l}0.55 \\
.65 \\
.65 \\
.7 \\
1.3\end{array}$ & $\begin{array}{l}0.35 \\
.25 \\
.25 \\
.4 \\
1.7\end{array}$ & $\begin{array}{l}0.2 \\
.25 \\
.25 \\
.2 \\
.4\end{array}$ & $\begin{array}{l}0.4 \\
.5 \\
.4 \\
.35 \\
.35\end{array}$ \\
\hline $\begin{array}{c}7 \ldots \\
8 \ldots \\
9 \ldots \ldots \\
10 \ldots\end{array}$ & $\begin{array}{r}.45 \\
.7 \\
.7 \\
.5 \\
2.5\end{array}$ & $\begin{array}{r}.5 \\
1.0 \\
.5 \\
.4 \\
1.0\end{array}$ & $\begin{array}{l}.2 \\
.35 \\
.5 \\
.35 \\
.9\end{array}$ & $\begin{array}{l}.15 \\
.15 \\
.15 \\
.15 \\
.15\end{array}$ & 1.3 & .1 & $\begin{array}{l}.35 \\
.25 \\
.2\end{array}$ & $\begin{array}{l}.08 \\
.08 \\
.07 \\
.08 \\
.07\end{array}$ & $\begin{array}{l}1.4 \\
.7 \\
1.7 \\
.9 \\
1.1\end{array}$ & $\begin{array}{l}.7 \\
.55 \\
.55 \\
.4 \\
.4\end{array}$ & $\begin{array}{l}.4 \\
.25 \\
.2 \\
.16 \\
.2\end{array}$ & $\begin{array}{l}.25 \\
.25 \\
.3 \\
.2 \\
.35\end{array}$ \\
\hline $\begin{array}{l}11 \\
12 \\
13 \\
15 \\
15\end{array}$ & $\begin{array}{l}2.3 \\
.9 \\
.65 \\
.65 \\
.5\end{array}$ & $\begin{array}{r}.6 \\
1.8 \\
1.0 \\
.9 \\
.7\end{array}$ & $\begin{array}{l}1.4 \\
.55 \\
.4 \\
.35 \\
.25\end{array}$ & $\begin{array}{l}.15 \\
.15 \\
.15 \\
.1 \\
.1\end{array}$ & & .5 & $\begin{array}{l}.15 \\
.2 \\
.2 \\
.2 \\
.35\end{array}$ & $\begin{array}{l}.09 \\
.09 \\
.09 \\
.25 \\
.6\end{array}$ & $\begin{array}{l}.9 \\
1.7 \\
1.1 \\
1.1 \\
2.1\end{array}$ & $\begin{array}{l}.35 \\
.25 \\
.25 \\
.2 \\
.25\end{array}$ & $\begin{array}{r}.16 \\
.09 \\
.16 \\
.16\end{array}$ & $\begin{array}{l}.2 \\
.2 \\
.3 \\
.2 \\
.2\end{array}$ \\
\hline $\begin{array}{l}16 \\
17 \\
18 \\
190 \\
20\end{array}$ & $\begin{array}{c}.45 \\
1.2 \\
.75 \\
.55 \\
.45\end{array}$ & $\begin{array}{r}.6 \\
.8 \\
2.5 \\
.9 \\
.9\end{array}$ & $\begin{array}{l}.3 \\
.35 \\
.4 \\
.5 \\
.3\end{array}$ & $\begin{array}{r}.1 \\
.09 \\
.09 \\
.09 \\
.09\end{array}$ & .7 & 2.4 & $\begin{array}{l}.65 \\
1.3 \\
1.2 \\
.85 \\
5.1\end{array}$ & $\begin{array}{l}.5 \\
3.4 \\
1.0 \\
2.6 \\
1.8\end{array}$ & $\begin{array}{l}.85 \\
.65 \\
.65 \\
.5 \\
.45\end{array}$ & $\begin{array}{l}.25 \\
.15 \\
1.3 \\
.7 \\
.55\end{array}$ & & $\begin{array}{l}.3 \\
.25 \\
.2 \\
.2 \\
.2\end{array}$ \\
\hline $\begin{array}{l}21 \\
22 \\
24 \\
25\end{array}$ & $\begin{array}{l}.4 \\
.4 \\
.4 \\
.35 \\
.25\end{array}$ & $\begin{array}{l}.7 \\
.55 \\
.5 \\
1.2 \\
.7\end{array}$ & $\begin{array}{l}.25 \\
.2 \\
.2 \\
.2 \\
.25\end{array}$ & $\begin{array}{l}a .07 \\
0.06 \\
.06 \\
.05 \\
.04\end{array}$ & & .6 & $\begin{array}{l}1.1 \\
.35 \\
.25 \\
.2 \\
.15\end{array}$ & $\begin{array}{l}.9 \\
1.8 \\
1.7 \\
1.4 \\
.75\end{array}$ & $\begin{array}{l}.4 \\
.4 \\
.4 \\
.5 \\
.65\end{array}$ & $\begin{array}{l}.45 \\
.4 \\
.35 \\
.35 \\
.35\end{array}$ & .1 & $\begin{array}{l}.2 \\
.15 \\
.1 \\
.15 \\
.2\end{array}$ \\
\hline $\begin{array}{l}26 \\
27 \\
29 \\
30 \\
311\end{array}$ & \begin{tabular}{c|}
.3 \\
1.2 \\
.85 \\
.5 \\
.4 \\
.35
\end{tabular} & $\begin{array}{l}.6 \\
.55 \\
.45 \\
.5 \\
.4 \\
.4\end{array}$ & $\begin{array}{l}.2 \\
.15 \\
.15 \\
.1 \\
.45\end{array}$ & $\begin{array}{r}.03 \\
.03 \\
.03 \\
.06 \\
.15 \\
0.06\end{array}$ & .3 & & $\begin{array}{l}.15 \\
.15 \\
.2 \\
.15 \\
.1 \\
.09 \\
-\end{array}$ & 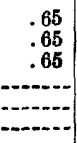 & $\begin{array}{l}.6 \\
.45 \\
.4 \\
.3 \\
.3 \\
.3\end{array}$ & $\begin{array}{l}.2 \\
.2 \\
.2 \\
.2 \\
.35\end{array}$ & $\begin{array}{l}1.5 \\
.6 \\
.55 \\
.5 \\
.5\end{array}$ & $\begin{array}{l}.15 \\
.1 \\
.1 \\
.1 \\
.2 \\
.:\end{array}$ \\
\hline \multirow{2}{*}{\multicolumn{4}{|c|}{ Month }} & \multicolumn{4}{|c|}{ Million gallons a day } & \multirow{2}{*}{\multicolumn{2}{|c|}{$\begin{array}{c}\text { Second- } \\
\text { feet } \\
\text { (mean) }\end{array}$}} & \multicolumn{3}{|c|}{ Total run-off } \\
\hline & & & & Maximum & Min & imum & Mean & & & $\begin{array}{l}\text { Million } \\
\text { gallons }\end{array}$ & \multicolumn{2}{|c|}{ Acre-feet } \\
\hline 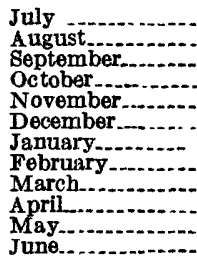 & 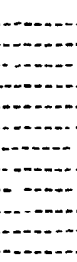 & $\ldots$ & & $\begin{array}{r}2.5 \\
2.5 \\
1.4 \\
2.3 \\
2.5 \\
-5.1 \\
3.4 \\
2.1 \\
1.7 \\
1.5 \\
.5\end{array}$ & & $\begin{array}{l}0.25 \\
.3 \\
.1 \\
.03 \\
.05 \\
.09 \\
.07 \\
.3 \\
.15 \\
.09 \\
.1\end{array}$ & $\begin{array}{r}0.740 \\
.774 \\
.358 \\
.116 \\
.637 \\
.70 \\
.561 \\
.704 \\
.785 \\
.428 \\
.255 \\
.235\end{array}$ & \multicolumn{2}{|c|}{$\begin{array}{l}1.14 \\
1.20 \\
.554 \\
.179 \\
.986 \\
1.08 \\
.868 \\
1.09 \\
1.21 \\
.662 \\
.395 \\
.364\end{array}$} & $\begin{array}{l}23.0 \\
24.0 \\
10.8 \\
3.60 \\
19.1 \\
21.6 \\
17.4 \\
19.7 \\
24.4 \\
12.8 \\
7.89 \\
7.05\end{array}$ & \multicolumn{2}{|r|}{$\begin{array}{l}70 \\
74 \\
33 \\
11 \\
59 \\
67 \\
53 \\
60 \\
75 \\
39 \\
24 \\
22\end{array}$} \\
\hline The year. & $\cdots$ & & & 5.1 & & .03 & .524 & & .811 & 191 & & $\overline{587}$ \\
\hline
\end{tabular}

- Partly estimated. 
MISCELIANEOUS MEASURRMENTS

Measurements of streams and ditches on the island of Oahu at other than regular gaging stations are listed below.

Miscellaneous discharge measurements on Oahu, 1928-29

\begin{tabular}{|c|c|c|c|c|c|}
\hline \multirow[b]{2}{*}{ Date } & \multirow[b]{2}{*}{ Stream } & \multirow[b]{2}{*}{ Tributary to- } & \multirow[b]{2}{*}{ Locality } & \multicolumn{2}{|c|}{ Discharge } \\
\hline & & & & $\begin{array}{l}\text { Sec- } \\
\text { ond- } \\
\text { feet }\end{array}$ & $\begin{array}{l}\text { Million } \\
\text { gallons } \\
\text { a day }\end{array}$ \\
\hline Jan. 24 & $\begin{array}{l}\text { Hiilaniwai Stream....-. } \\
\text { Luluku Stregm }\end{array}$ & Luluku Stream....... & $\begin{array}{l}\text { Elevation } 460 \text { feet, opposite } \\
\text { Kailua water system's in- } \\
\text { take, near Kaneohe. } \\
1.000 \text { feet below Kailua wa- }\end{array}$ & 0.39 & 0.25 \\
\hline 24 & First Division ditch... & Kaneobe stream. & $\begin{array}{l}\text { 1,00 feet below Kallua wa- } \\
\text { ter system's intake, near } \\
\text { Kaneohe. } \\
50 \text { feet below intake near }\end{array}$ & .12 & .078 \\
\hline 24 & Diversion ditch of & _.....do..... & 300 feet above Kaneohe & .11 & .07 \\
\hline 24 & Luluku Stream........... & Keneohe Stream. & $\begin{array}{l}\text { 1,000 feet above confluence } \\
\text { with Kaneohe Stream, } \\
\text { near Kaneohe. }\end{array}$ & .82 & .53 \\
\hline 24 & North Luluku ditch.... & Taro patches.- & $\begin{array}{l}\text { Last main diversion on } \\
\text { Waiahole side of Luluku } \\
\text { Stream, near Kaneohe. }\end{array}$ & .80 & .52 \\
\hline Mar. 28 & North Halawa Stream. & Pacific Ocean & $\begin{array}{l}\text { Elevation } 300 \text { foet, near } \\
\text { Aiea. }\end{array}$ & .6 & .39 \\
\hline
\end{tabular}

Notk. - In addition to the above miscellaneous measurements 492 measurements were made by J. F. Kunesh or under his supervision. These measurements will be published in a supplement to the report of the Board of Water Supply, city and county of Honolulu, to the legislature of the Territory of Hawaii, sixteenth regular session, under the title " Water Resources of the City of Honolulu, 1928-1930." 


\section{ISLAND OF MOLOKAI}

\section{HALAWA STREAM NEAR HALAWA, MOLOKAI}

Location.-Water-stage recorder 750 feet below confluence of two main branches and 2 miles above mouth of stream and Halawa schoolhouse.

Drain age arta.-4.6 square miles.

Records avarlable.-August, 1917, to June, 1929.

ExTREmes.-Maximum discharge recorded during year, 431 million gallons a day or 667 second-feet Apr. 18 (gage height, 5.65 feet); minimum, 2.9 million gallons a day or 4.5 second-feet June 24 .

1918-1929: Maximum discharge, about 1,550 million gallons a day or 2,400 second-feet Mar. 31, 1923 (gage height, 11.65 feet); minimum, 0.8 million gallons a day or 1.2 second-feet Oct. 13-15, 19, 1917.

A maximum discharge greater than 1,550 million gallons a day may have occurred Jan. 20, 1929.

Remarks.-Records poor. 1-inch pipe line diverts water one-fourth mile above station for domestic use.

Discharge, in million gallons a day, 1928-29

\begin{tabular}{|c|c|c|c|c|c|c|c|c|c|c|c|c|c|}
\hline Day & July & Aug. & Sept. & Oct. & No & v. & Dec. & Jan. & Feb. & Mar. & A pr. & May & June \\
\hline $\begin{array}{l}1 \\
2 \\
3 \\
4 \\
5\end{array}$ & $\begin{array}{l}28 \\
36 \\
17.8 \\
13.1 \\
11.3\end{array}$ & 24 & $\begin{array}{r}8.4 \\
8.2 \\
a 8.7 \\
17\end{array}$ & $\begin{array}{r}20 \\
8.0 \\
9.1 \\
12.1\end{array}$ & & 3.5 & 4. 3 & 12 & 4.0 & $\begin{array}{l}12 \\
14 \\
52 \\
86\end{array}$ & $\begin{array}{l}10 \\
51 \\
14.5\end{array}$ & $\begin{array}{l}7.6 \\
8.1 \\
5.9 \\
5.2 \\
4.7\end{array}$ & $\begin{array}{l}5.5 \\
5.2 \\
5.9 \\
5.5 \\
4.3\end{array}$ \\
\hline $\begin{array}{r}6 \\
7 \\
8 \\
9\end{array}$ & $\begin{array}{l}9.6 \\
19.7 \\
29 \\
12.5 \\
24\end{array}$ & 14 & 13 & $\begin{array}{c}9.4 \\
9.1 \\
8.0 \\
37 \\
48\end{array}$ & $\int_{b 36}$ & & 32 & 90 & 14 & $\begin{array}{l}35 \\
19.5 \\
22 \\
12 \\
15\end{array}$ & $\begin{array}{c}8.2 \\
8.2 \\
9.3 \\
6.2 \\
26\end{array}$ & $\begin{array}{l}9.8 \\
6.8 \\
9.5 \\
5.9 \\
4.7\end{array}$ & $\begin{array}{l}5.0 \\
5.0 \\
4.7 \\
4.0 \\
8.0\end{array}$ \\
\hline $\begin{array}{l}11 \\
12 \\
13 \\
14 \\
15\end{array}$ & $\begin{array}{l}33 \\
\text { b 12.1 } \\
\text { a } 11.0 \\
\text { a } 12.1 \\
11\end{array}$ & . & 11 & $\begin{array}{r}14.7 \\
12.9 \\
15.6 \\
a 15.1 \\
11\end{array}$ & 14 & & 30 & 5.5 & 14 & $\begin{array}{l}38 \\
32\end{array}$ & $\begin{array}{c}12 \\
8.2 \\
6.5 \\
5.5 \\
7.5\end{array}$ & $\begin{array}{l}5.0 \\
4.7 \\
5.0 \\
7.0 \\
9.1\end{array}$ & $\begin{array}{r}6.2 \\
5.5 \\
8.5 \\
5.9 \\
11.5\end{array}$ \\
\hline $\begin{array}{l}16 \\
17 \\
18 \\
19 \\
20\end{array}$ & $\left\{\begin{array}{l}33 \\
13\end{array}\right.$ & 26 & 28 & & 22 & & & 9 & 5.5 & $\begin{array}{r}8 \\
44\end{array}$ & $\begin{array}{r}5.9 \\
5.0 \\
123 \\
19.5 \\
11.5\end{array}$ & $\begin{array}{l}5.2 \\
4.3 \\
4.3 \\
4.0 \\
3.8\end{array}$ & $\begin{array}{r}8.2 \\
5.0 \\
15 \\
5.2 \\
4.3\end{array}$ \\
\hline $\begin{array}{l}21 \ldots . \\
22 \\
23 \\
24 \ldots \\
25 \ldots\end{array}$ & 22 & $\begin{array}{l}28 \\
20 \\
12.1 \\
23 \\
12.5\end{array}$ & 6 & 4.9 & $\begin{array}{r}14 \\
7 \\
7 \\
12\end{array}$ & \begin{tabular}{l|}
4.5 \\
7.8 \\
7.2 \\
2.7
\end{tabular} & 15 & 7.5 & 28 & 11 & $\begin{array}{c}7.5 \\
6.5 \\
6.2 \\
14 . \\
6.8\end{array}$ & $\begin{array}{l}3.6 \\
3.6 \\
4.3 \\
5.2 \\
4.0\end{array}$ & $\begin{array}{r}3.8 \\
3.6 \\
3.1 \\
3.8 \\
44\end{array}$ \\
\hline $\begin{array}{l}26 \\
28 \\
28 \\
28 \\
31\end{array}$ & $\begin{array}{l}75 \\
17\end{array}$ & $\begin{array}{r}11.0 \\
12.8 \\
11.0 \\
11.0 \\
17.1 \\
9.6\end{array}$ & & $\begin{array}{l}70 \\
8.5\end{array}$ & & 5 & $\begin{array}{l}6 \\
9.5\end{array}$ & $\begin{array}{r}18 \\
6\end{array}$ & .... & 6 & \begin{tabular}{c}
5.2 \\
5.0 \\
8.6 \\
19 \\
5.5 \\
\hdashline...
\end{tabular} & $\begin{array}{c}9.2 \\
47 \\
16 \\
8.9 \\
9.3 \\
13\end{array}$ & $\begin{array}{r}16 \\
5.5 \\
4.5 \\
4.0 \\
3.6 \\
-2\end{array}$ \\
\hline \multirow{2}{*}{\multicolumn{4}{|c|}{ Month }} & \multicolumn{5}{|c|}{ Million gallons a day } & \multirow{2}{*}{\multicolumn{2}{|c|}{$\begin{array}{c}\text { Second- } \\
\text { feet } \\
\text { (mean) }\end{array}$}} & \multicolumn{3}{|c|}{ Total run-off } \\
\hline & & & & \multicolumn{2}{|c|}{ Maximum } & \multicolumn{2}{|c|}{ Minimum } & Mean & & & $\begin{array}{l}\text { Million } \\
\text { gallons }\end{array}$ & \multicolumn{2}{|c|}{ Acre-feet } \\
\hline $\begin{array}{l}\text { July... } \\
\text { August } \\
\text { September-. } \\
\text { October } \\
\text { November-.. } \\
\text { December } \\
\text { January } \\
\text { February } \\
\text { March } \\
\text { April } \\
\text { May. } \\
\text { June }\end{array}$ & 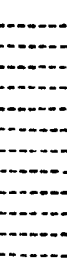 & . & -.. & 12 & $\begin{array}{l}- \\
23 \\
4 \\
4\end{array}$ & - & $\begin{array}{l} \\
5.0 \\
3.6 \\
3.1\end{array}$ & $\begin{array}{c}23.7 \\
17.4 \\
13.4 \\
15.5 \\
11.6 \\
17.9 \\
24.1 \\
11.8 \\
21.6 \\
14.7 \\
7.89 \\
7.34\end{array}$ & & $\begin{array}{l}36.7 \\
26.9 \\
20.7 \\
24.0 \\
17.9 \\
27.7 \\
37.3 \\
18.3 \\
33.4 \\
22.7 \\
12.2 \\
11.4\end{array}$ & $\begin{array}{l}\mathbf{7 3 5} \\
\mathbf{5 3 8} \\
401 \\
480 \\
349 \\
\mathbf{5 5 5} \\
748 \\
329 \\
668 \\
442 \\
245 \\
220\end{array}$ & & $\begin{array}{r}2,250 \\
1,660 \\
1,230 \\
1,470 \\
1,070 \\
1,700 \\
2,290 \\
1,010 \\
2,050 \\
1,350 \\
751 \\
676\end{array}$ \\
\hline The year. & 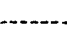 & & & & & & & 15.6 & & $\overline{24.1}$ & 5,710 & & $\overline{17,500}$ \\
\hline
\end{tabular}


PAPALAUA STREAM NEAR WATHE, MOLOKAI

LocAtion.- Water-stage recorder a quarter of a mile above mouth, 2 miles east of Wailau landing, and $6 \frac{1}{2}$ miles due north of Pukoo village.

Drainage area. - 2.0 square miles.

RECORDS AVAIIABLE. - September, 1919, to September, 1929. (diseontinued).

Extremes.-Maximum discharge during period, 976 million gallons a day or 1,510 second-feet Jan. 20 (gage height, 7.78 feet); minimum, 1.2 million gallons a day or 1.9 second-feet July $15,1929$.

1920-1929: Maximum discharge, about 1,140 million gallons a day or 1,760 second-feet Dec. 24, 1920 (gage height, 8.58 feet); minimum, 0.9 million gallons a day or 1.4 second-feet July $1,5,6,1926$.

REMARKs.-Records good for ordinary stages; poor for extremely high stages. No diversion.

Daily discharge, in million gallons a day, 1928-29

\begin{tabular}{|c|c|c|c|c|c|c|c|c|c|c|c|c|c|}
\hline \multicolumn{2}{|l|}{ Day } & uly & ug. & Sept. & Oct. & Nov. & Deo. & Jan. & Feb. & Mar. & Apr. & May & Jur \\
\hline \multicolumn{2}{|c|}{ 5 } & $\begin{array}{l}23 \\
27 \\
12.2 \\
8.0 \\
6.5\end{array}$ & $\begin{array}{l}27 \\
13.1 \\
23 \\
6.9 \\
6.8\end{array}$ & $\begin{array}{r}4.5 \\
4.2 \\
17.6 \\
11.8 \\
12.4\end{array}$ & $\begin{aligned} 24 \\
13.6 \\
4.5 \\
6.0 \\
9.6\end{aligned}$ & \begin{tabular}{l|}
9.0 \\
5.2 \\
3.5 \\
5.4 \\
8.0
\end{tabular} & \begin{tabular}{r|}
3.7 \\
3.9 \\
3.4 \\
3.5 \\
78
\end{tabular} & $\begin{array}{r}6.9 \\
14.8 \\
5.2 \\
4.4 \\
4.8\end{array}$ & 2. & $\begin{array}{r}7.0 \\
13.9\end{array}$ & $\begin{array}{r}6 . \\
7 .\end{array}$ & & \\
\hline \multicolumn{2}{|c|}{ (6-10. } & $\begin{array}{r}5.6 \\
17.3 \\
25 \\
9.0 \\
18.5\end{array}$ & $\begin{array}{r}10.3 \\
17.5 \\
5.8 \\
4.6 \\
9.7\end{array}$ & $\begin{array}{r}5.1 \\
7.0 \\
8.2 \\
19.8 \\
26\end{array}$ & $\begin{array}{l}5.8 \\
6.0 \\
4.5 \\
47 \\
41\end{array}$ & \begin{tabular}{c|}
9.8 \\
33 \\
10.1 \\
12.7 \\
6.0
\end{tabular} & \begin{tabular}{c|}
25 \\
5.6 \\
6.5 \\
36 \\
17.8
\end{tabular} & $\begin{array}{r}4.5 \\
4.2 \\
86 \\
6.5 \\
4.4\end{array}$ & & & D & & \\
\hline \multicolumn{2}{|c|}{$\begin{array}{l}11 \\
112 \\
13 \\
15\end{array}$} & \begin{tabular}{c|}
40 \\
7.7 \\
5.6 \\
7.4 \\
4.8
\end{tabular} & $\begin{array}{r}16.9 \\
23 \\
8.2 \\
11.1 \\
5.8\end{array}$ & $\begin{aligned} 53 \\
11.0 \\
7.3 \\
5.8 \\
4.8\end{aligned}$ & $\begin{array}{l}11.2 \\
15.0 \\
13.6 \\
12.6 \\
15.0\end{array}$ & & $\begin{array}{l}6,0 \\
8.0 \\
3 \\
6\end{array}$ & $\begin{array}{r}4.0 \\
3.0 \\
2.7 \\
12.9 \\
19.0\end{array}$ & $\begin{array}{r}2 . \\
2 . \\
2 . \\
17 . \\
9 .\end{array}$ & $\begin{array}{l}38 \\
77 \\
73.3\end{array}$ & & & \\
\hline \multicolumn{2}{|c|}{10} & \begin{tabular}{r|}
8.1 \\
32 \\
8.4 \\
9.6 \\
7.4
\end{tabular} & $\begin{array}{l}18.5 \\
40 \\
25 \\
7.1 \\
28\end{array}$ & $\begin{array}{l}4.2 \\
12.0 \\
26 \\
40 \\
10.6\end{array}$ & $\begin{array}{l}5.8 \\
4.5 \\
4.0 \\
5.1 \\
3.7\end{array}$ & & \begin{tabular}{r|}
64 \\
15.3 \\
8.5 \\
6.7 \\
5.6
\end{tabular} & \begin{tabular}{l|}
24 \\
10.0 \\
5.1 \\
28 \\
69
\end{tabular} & $\begin{array}{l}32 \\
89 \\
21 \\
72 \\
28\end{array}$ & $\begin{array}{c}6.9 \\
5.4 \\
6.2 \\
66 \\
17.0\end{array}$ & $\begin{array}{r}5.0 \\
4.0 \\
122 \\
18.1 \\
9.5\end{array}$ & & 18 \\
\hline \multicolumn{2}{|c|}{221} & \begin{tabular}{r|}
12.3 \\
30 \\
9.4 \\
14.7 \\
52
\end{tabular} & $\begin{array}{l}21 \\
20 \\
8.0 \\
16.6 \\
9.0\end{array}$ & $\begin{array}{l}6.2 \\
4.8 \\
3.8 \\
3.5 \\
5.2\end{array}$ & $\begin{array}{l}3.3 \\
3.1 \\
2.9 \\
5.0 \\
3.1\end{array}$ & \begin{tabular}{r|}
10.6 \\
6.0 \\
5.1 \\
10.4 \\
5.6
\end{tabular} & $\begin{array}{r}5.6 \\
6.9 \\
5.6 \\
11.4 \\
21\end{array}$ & \begin{tabular}{|c|}
123 \\
174 \\
17.3 \\
8.0 \\
6.0
\end{tabular} & 7.5 & $\begin{array}{r}12.0 \\
6.7 \\
9.2 \\
13.2 \\
17.4\end{array}$ & $\begin{array}{l}6 . \\
4 . \\
4\end{array}$ & & $\begin{array}{r}2 . \\
2 . \\
5 . \\
42\end{array}$ \\
\hline \multicolumn{2}{|c|}{$\begin{array}{l}26 \\
27 \\
28 \\
20 \\
30 \\
31\end{array}$} & $\begin{aligned} 54 \\
100 \\
17.5 \\
9.7 \\
6.9 \\
5.6\end{aligned}$ & $\begin{array}{r}7.5 \\
7.5 \\
6.2 \\
10.4 \\
14.3 \\
5.6\end{array}$ & $\begin{array}{l}3.8 \\
2.8 \\
3.8 \\
2.9 \\
2.6\end{array}$ & \begin{tabular}{c|}
2.5 \\
108 \\
33 \\
6.4 \\
5.0 \\
4.0
\end{tabular} & \begin{tabular}{l|}
4.5 \\
3.8 \\
3.4 \\
3.4 \\
3.5
\end{tabular} & $\begin{array}{r}8.6 \\
5.0 \\
4.8 \\
13.9 \\
5.4 \\
4.8\end{array}$ & $\begin{array}{r}5.9 \\
9.6 \\
23 \\
6.4 \\
4.5 \\
3.7\end{array}$ & $\begin{array}{r}18.2 \\
10.0 \\
8.7\end{array}$ & $\begin{array}{r}11.2 \\
5.8 \\
4.6 \\
4.2 \\
3.5 \\
3.7\end{array}$ & $\begin{array}{r}3.9 \\
3.5 \\
14.3 \\
21 \\
5.0\end{array}$ & $\begin{array}{r}9 . \\
5 . \\
7 . \\
15 .\end{array}$ & $\begin{array}{r}11 . \\
3 . \\
3 . \\
2 .\end{array}$ \\
\hline Day & July & Aug. & Sept. & & $y$ & July & Aug. & Sept. & & & July & ing. & Sept \\
\hline 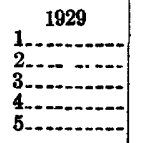 & $\begin{array}{l}2.6 \\
3.7 \\
2.5 \\
2.0 \\
1.8\end{array}$ & $\begin{array}{r}7.4 \\
7.1 \\
10.2 \\
9.7 \\
6.0\end{array}$ & $\begin{array}{l}4.2 \\
5.1\end{array}$ & $\begin{array}{l}2 \\
1\end{array}$ & 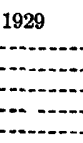 & $\begin{array}{l}1.7 \\
1.6\end{array}$ & $\begin{array}{r}16.0 \\
8.0 \\
6.7 \\
5.4 \\
5.4\end{array}$ & & $\begin{array}{l}21 . \\
22 . \\
23 . \\
24 . \\
25 .\end{array}$ & & $\begin{array}{r}5.9 \\
6.4 \\
4.6 \\
10.4 \\
29\end{array}$ & 5. & $\cdots$ \\
\hline $\begin{array}{r}7 \\
8 \\
9\end{array}$ & $\begin{array}{l}1.6 \\
4.8 \\
2.6 \\
1.7 \\
2.5\end{array}$ & $\begin{array}{l}6.9 \\
6.9 \\
4.8 \\
22 \\
20\end{array}$ & & $\begin{array}{l}16 \\
17 . \\
18 . \\
19 . \\
20\end{array}$ & (1) & $\begin{array}{l}35 \\
6.0 \\
4.2 \\
2.7 \\
3.7\end{array}$ & $\begin{array}{l}8.3 \\
7.1 \\
5.1 \\
5.1 \\
4.8\end{array}$ & 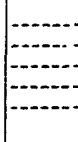 & $\begin{array}{l}26 . \\
27 . \\
28 . \\
29 . \\
30 .\end{array}$ & & $\begin{array}{l}68 \\
14.3 \\
7.8 \\
5.1 \\
4.4 \\
5.4\end{array}$ & $\begin{array}{r}11.9 \\
5.1 \\
3.9 \\
3.1 \\
2.7 \\
11.6\end{array}$ & 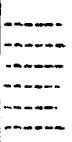 \\
\hline
\end{tabular}


Monthly discharge of Papalaua Stream near Wailau, Molokai, 1928-29

\begin{tabular}{|c|c|c|c|c|c|c|}
\hline \multirow{2}{*}{ Month } & \multicolumn{3}{|c|}{ Million gallons a day } & \multirow{2}{*}{$\begin{array}{l}\text { Second- } \\
\text { feet } \\
\text { (mean) }\end{array}$} & \multicolumn{2}{|c|}{ Total run-off } \\
\hline & Maximum & Minimum & Mean & & $\begin{array}{l}\text { Million } \\
\text { gallons }\end{array}$ & Acre-feet \\
\hline $\begin{array}{l}\text { Juhy } \\
\text { August } \\
\text { September } \\
\text { October } \\
\text { November. } \\
\text { December } \\
\text { January } \\
\text { Pebruary } \\
\text { March } \\
\text { April } \\
\text { May } \\
\text { June }\end{array}$ & $\begin{array}{r}100 \\
40 \\
58 \\
108 \\
33 \\
78 \\
174 \\
89 \\
66 \\
122 \\
19.2 \\
42\end{array}$ & $\begin{array}{l}4.8 \\
4.6 \\
2.6 \\
2.5 \\
3.4 \\
3,4 \\
2.7 \\
2.6 \\
3.5 \\
3.5 \\
2.7 \\
2.2\end{array}$ & $\begin{array}{r}19.2 \\
14.0 \\
11.0 \\
13.8 \\
9.54 \\
16.1 \\
22,6 \\
17.1 \\
18.4 \\
15.1 \\
6.34 \\
7.15\end{array}$ & $\begin{array}{l}29.7 \\
21.7 \\
17.0 \\
21.4 \\
14.8 \\
24,9 \\
35.0 \\
26.5 \\
28.5 \\
23.4 \\
9.81 \\
11.1\end{array}$ & $\begin{array}{l}595 \\
434 \\
331 \\
429 \\
286 \\
498 \\
701 \\
478 \\
571 \\
453 \\
196 \\
214\end{array}$ & $\begin{array}{r}1,830 \\
1,330 \\
1,010 \\
1,310 \\
878 \\
1,550 \\
2,150 \\
1,470 \\
1,750 \\
1,390 \\
603 \\
658\end{array}$ \\
\hline . The year.. & 174 & 2.2 & 14.2 & 22.0 & 5,190 & 15,900 \\
\hline $\begin{array}{l}\text { July } \\
\text { August } \\
\text { Ang }\end{array}$ & $\begin{array}{l}68 \\
22\end{array}$ & $\begin{array}{l}1.3 \\
2.7\end{array}$ & $\begin{array}{l}8.01 \\
8.07\end{array}$ & $\begin{array}{l}12.4 \\
12.5\end{array}$ & $\begin{array}{l}248 \\
250\end{array}$ & $\begin{array}{l}762 \\
768\end{array}$ \\
\hline
\end{tabular}

$67754-32-4$ 
WAIAKEAKUA STREAM NEAR WAIIAU, MOLOKAI

Locatron.-Water-stage recorder half a mile above confluence with Pulena Stream, 3 miles south of Wailau landing, and 4 miles northwest of Pukoo village.

Drainage area.-1.4 square miles.

Records aVAILABLe. - October, 1919, to September, 1929 (discontinued).

ExTremes.-Maximum discharge during period, 582 million gallons a day or 900 second-feet Jan. 20 (gage height, 6.51 feet); minimum, 1.7 million gallons a day or 2.6 second-feet July 14, 1929.

1920 1929: Maximum discharge, about 710 million gallons a day or 1,100 second-feet Mar. 31, 1923 (gage height, 7.15 feet); minimum, 1.3 million gallons a day or 2.0 second-feet Mar. 7, 1920.

REMARKs.-Records good for ordinary stages, fair for estimated periods, and poor for high stages. No diversions.

Daily discharge, in million gallons a day, 1928-29

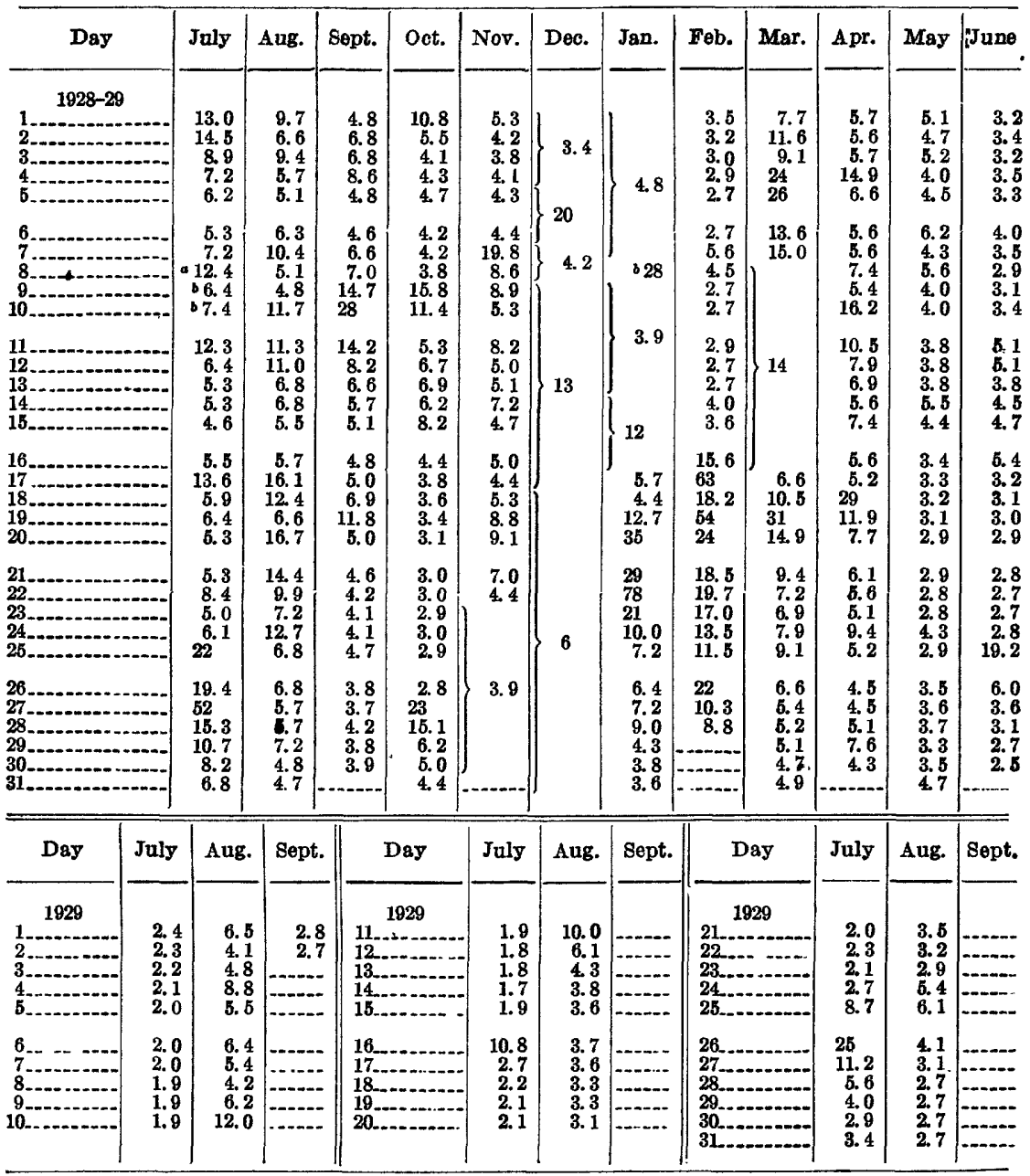

a Partly estimated.

- Estimated. 
Monthly discharge of Waiakeakua Stream near Wailau, Molokai, 1928-29

\begin{tabular}{|c|c|c|c|c|c|c|}
\hline \multirow{2}{*}{ Month } & \multicolumn{3}{|c|}{ Million gallons a day } & \multirow{2}{*}{$\begin{array}{l}\text { Second- } \\
\text { feet } \\
\text { (mean) }\end{array}$} & \multicolumn{2}{|c|}{ Total run-off } \\
\hline & Maximum & Minimum & Mean & & $\begin{array}{l}\text { Million } \\
\text { gallons }\end{array}$ & Acre-feet \\
\hline $\begin{array}{l}\text { July } \\
\text { August } \\
\text { September. } \\
\text { October. } \\
\text { November } \\
\text { December } \\
\text { January } \\
\text { February } \\
\text { March } \\
\text { April } \\
\text { May }\end{array}$ & \begin{tabular}{|c|}
52 \\
16.7 \\
28 \\
23 \\
\hdashline 78 \\
63 \\
\hdashline 29 \\
6.2 \\
19.2
\end{tabular} & \begin{tabular}{r}
4.6 \\
4.7 \\
3.7 \\
2.8 \\
\hdashline 2.7 \\
4.7 \\
4.3 \\
2.8 \\
2.5
\end{tabular} & $\begin{array}{l}10.3 \\
8.37 \\
6.90 \\
6.18 \\
5.80 \\
8.48 \\
11.4 \\
12.3 \\
11.9 \\
7.79 \\
3.96 \\
4.08\end{array}$ & $\begin{array}{r}15.9 \\
13.0 \\
10.7 \\
9.56 \\
8.97 \\
13.1 \\
17.6 \\
19.0 \\
18.4 \\
12.1 \\
6.13 \\
6.31\end{array}$ & $\begin{array}{l}318 \\
260 \\
207 \\
192 \\
174 \\
263 \\
354 \\
346 \\
368 \\
234 \\
123 \\
122\end{array}$ & $\begin{array}{r}980 \\
796 \\
635 \\
588 \\
534 \\
507 \\
\mathbf{8 0 7} \\
\mathbf{1 , 0 8 0} \\
\mathbf{1 , 0 6 0} \\
\mathbf{1 , 1 3 0} \\
\mathbf{7 1 7} \\
\mathbf{3 7 7} \\
\mathbf{3 7 6}\end{array}$ \\
\hline The year... & - & - & 8.11 & 12.5 & 2,960 & 9,080 \\
\hline $\begin{array}{l}1929 \\
\text { July }\end{array}$ & $\begin{array}{l}25 \\
12.0\end{array}$ & $\begin{array}{l}1.7 \\
2.7\end{array}$ & $\begin{array}{l}3.86 \\
4.77\end{array}$ & $\begin{array}{l}5.97 \\
7.38\end{array}$ & $\begin{array}{l}120 \\
148\end{array}$ & $\begin{array}{l}367 \\
454\end{array}$ \\
\hline
\end{tabular}


PULENA streaM NEaR WaILaU, MOLOKaI

Location.-Water-stage recorder half a mile above confluence with Waiakeakua Stream, 3 miles south of Wailau landing, and 4 miles northwest of Pukoo Village.

Drainage area.-4.4 square miles.

Records available. - October, 1919, to December, 1928 (discontinued).

Extremes. - Maximum gage height (determined from flood marks) at least 22 feet Jan. 20, 1929 (discharge undetermined); minimum, 6.8 million gallons a day or 10.5 second-feet Oct. $26,27$.

1920-1929: Maximum discharge, that of Jan. 20, 1923 (gage height, at least 22 feet); minimum, 3.0 million gallons a day or 4.6 second-feet June 28 and July 14, 1920 .

Remarks.-Records fair. No diversions. Station destroyed by flood of Jan. 20.

Discharge, in million gallons a day, 1928

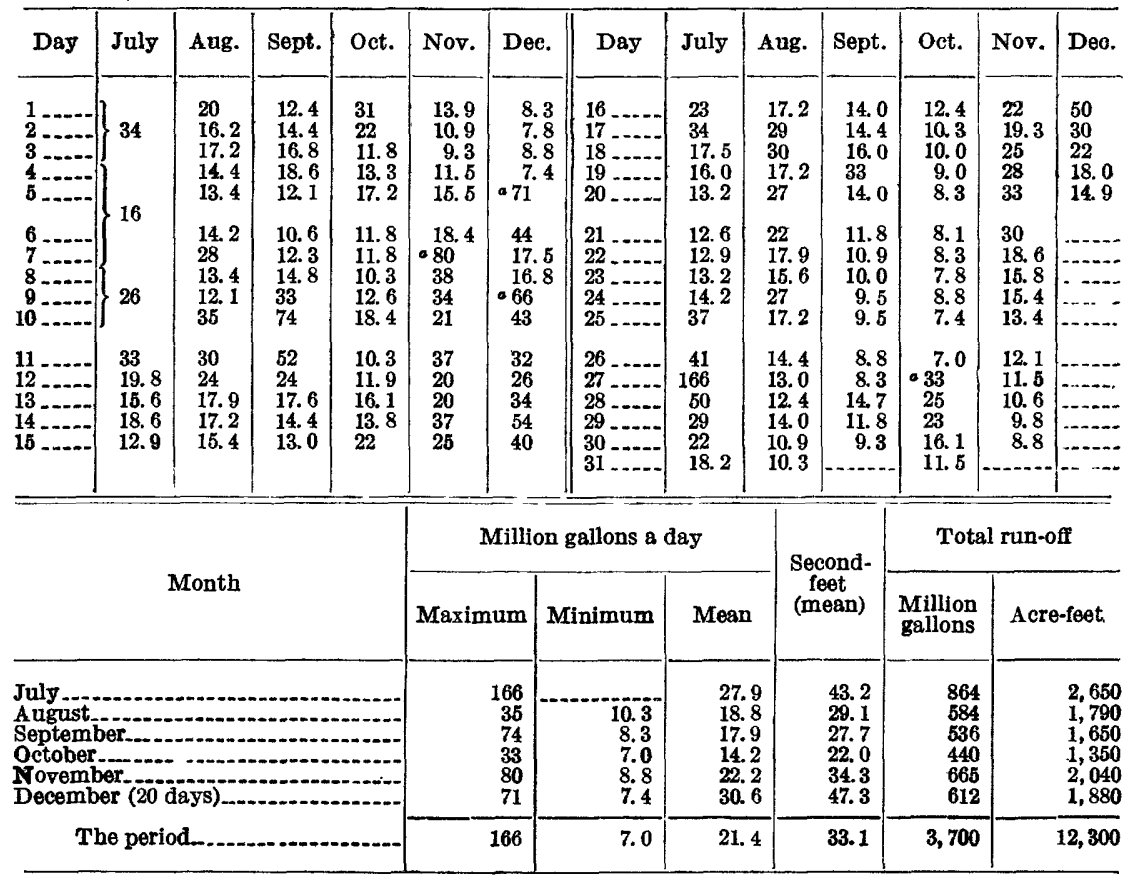

- Partly estimated. 


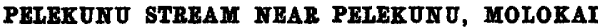

LOCATION.-Water-stage recorder half a mile above confluence with Lanipuni Stream, 2 miles south of Pelekunu landing, and 6 miles north of Kamalo village.

Drainage area.-2.4 square miles.

Ri CORDs AV AILABLE. - December, 1919, to January, 1929.

Extrames.-Maximum discharge during period, 1,330 million gallons a day or 2,060 second-feet Jan. 20 (gage height, 11.5 feet; estimated from floodmarks); minimum not known, owing to faulty record.

1920-1929: Maximum discharge, that of Jan. 20, 1929; minimum, 1.8 million gallons a day or 2.8 second-feet Mar. 7 and July 13,1920 .

REMARKs. - Records fair for ordinary stages except those estimated, which are poor. Records for extremely high stages poor. No diversions. Station destroyed by flood Jan. 20.

Discharge, in million gallons a day, 1928-29

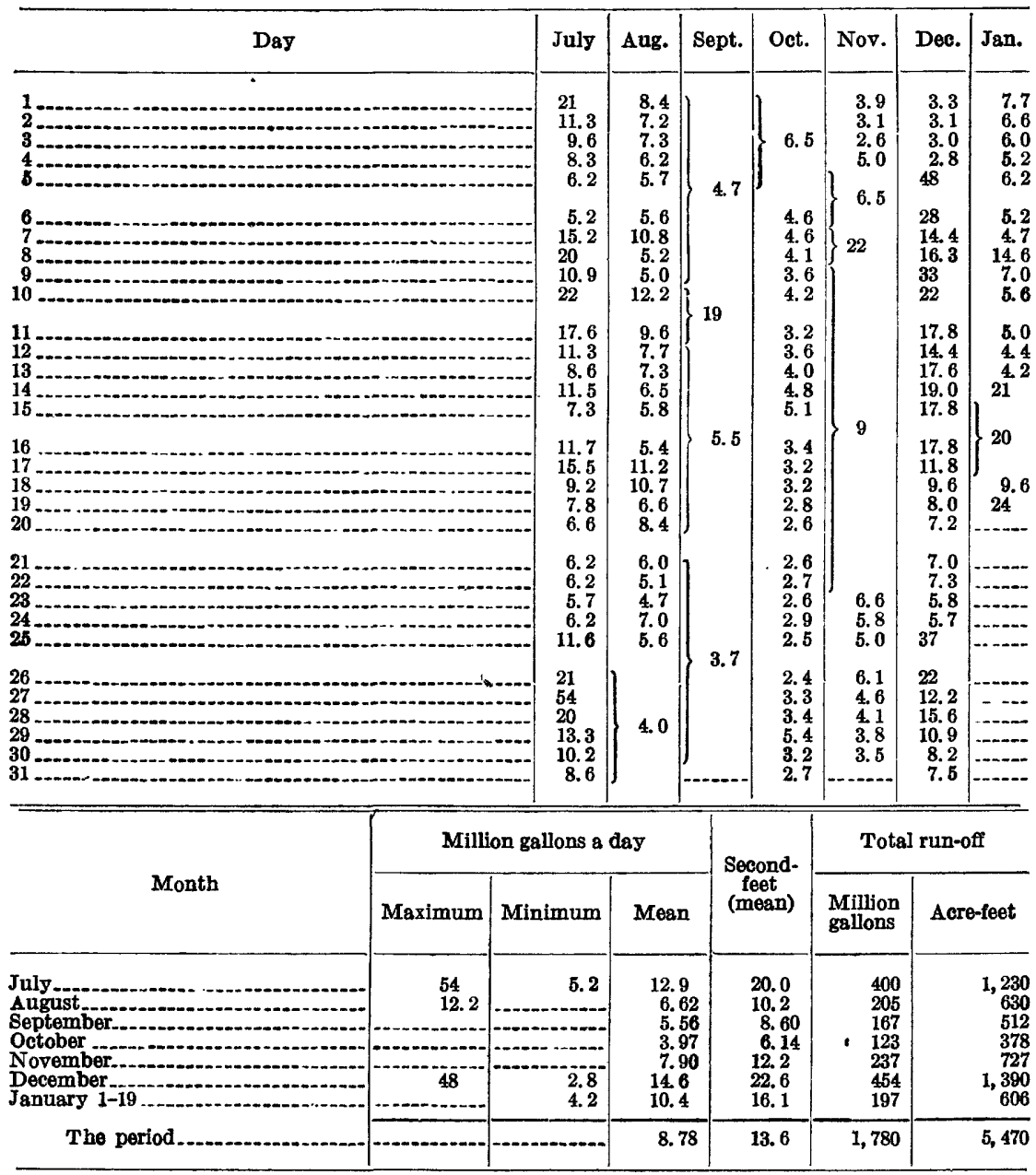




\section{IANIPUNI STREAM NEAR PMLEUNU, MOLOKAI}

Location.-Water-stage recorder half a mile above junction with Pelekunu Stream, 2 miles south of Pelekunu landing, and 6 miles north of Kamalo village.

Drainage area. - 0.9 square mile.

Records avatuable.-December, 1919, to September, 1929 (discontinued).

EXTREMES.-Maximum discharge during period, 938 million gallons a day or 1,450 second-feet Jan. 20 (gage height, 5.12 feet); minimum, 2.6 million gallons a day or 4.0 second-feet Dec. 4 .

1920-1929: Maximum discharge, about 1,250 million gallons a day or 1,930 second-feet Dec. 24, 1920 (gage height, 5.90 feet); minimum, 1.8 million gallons a day or 2.8 second-feet Nov. 10, 11, 1926 .

REMARKs.- Records good for ordinary stages; poor for extremely high stages. No diversions.

Daily discharge, in million gallons a day, 1928-29

\begin{tabular}{|c|c|c|c|c|c|c|c|c|c|c|c|c|c|}
\hline \multicolumn{2}{|l|}{ Day } & July & Aug. & Sept. & Oct. & Nov. & Dec. & Jan. & Feb. & Mar. & Apr. & May & June \\
\hline \multicolumn{2}{|l|}{$1928-29$} & \begin{tabular}{r|}
17.6 \\
9.9 \\
7.2 \\
5.7 \\
4.8
\end{tabular} & $\begin{array}{l}9.0 \\
5.7 \\
5.9 \\
5.2 \\
4.8\end{array}$ & $\begin{array}{l}4.0 \\
5.0 \\
5.4 \\
6.1 \\
\text { 4. } 2\end{array}$ & $\begin{array}{r}12.4 \\
6.2 \\
4.2 \\
5.0 \\
7.2\end{array}$ & $\begin{array}{l}\text { 3. } 6 \\
3.5 \\
\text { 3.4 } \\
6.2 \\
6.1\end{array}$ & $\begin{array}{r}2.8 \\
2.7 \\
2.8 \\
2.7 \\
131\end{array}$ & $\begin{array}{l}6.8 \\
6.4 \\
5.9 \\
5.4 \\
5.9\end{array}$ & $\begin{array}{l}\text { 5. } 6 \\
5.3 \\
5.2 \\
5.2 \\
5.2\end{array}$ & $\begin{array}{l}9.9 \\
18.4 \\
24 \\
59 \\
61\end{array}$ & $\begin{array}{l}4.8 \\
4.9 \\
4.8 \\
4.9 \\
4.5\end{array}$ & $\begin{array}{l}4.9 \\
4.4 \\
4.2 \\
4.2 \\
4.5\end{array}$ & $\begin{array}{l}4.6 \\
5.3 \\
4.5 \\
7.1 \\
4.9\end{array}$ \\
\hline \multicolumn{2}{|c|}{$\begin{array}{c}6 \\
8 \\
8 \\
0\end{array}$} & $\begin{array}{c}4.5 \\
11.8 \\
21 \\
7.7 \\
28\end{array}$ & $\begin{array}{r}4.8 \\
8.6 \\
4.7 \\
5.0 \\
12.7\end{array}$ & $\begin{array}{r}4.0 \\
4.0 \\
3.9 \\
8.3 \\
34 .\end{array}$ & $\begin{array}{l}4.5 \\
4.3 \\
4.0 \\
4.2 \\
4.7\end{array}$ & $\begin{array}{r}6.8 \\
47 \\
13.1 \\
9.1 \\
5.7\end{array}$ & $\begin{array}{l}53 \\
11.4 \\
21 \\
47 \\
19.6\end{array}$ & $\begin{array}{c}5.2 \\
5.2 \\
37 \\
8.5 \\
6.8\end{array}$ & $\begin{array}{l}5.2 \\
6.5 \\
6.6 \\
5.2 \\
5.1\end{array}$ & \begin{tabular}{l|}
23 \\
16.2 \\
17.0 \\
15.8 \\
10.2
\end{tabular} & $\begin{array}{r}4.5 \\
4.6 \\
6.4 \\
5.1 \\
27\end{array}$ & $\begin{array}{l}\text { 6. } 5 \\
\text { 5. } \\
5.9 \\
4.5 \\
\text { 5. }\end{array}$ & $\begin{array}{l}6.3 \\
5.1 \\
4.5 \\
4.3 \\
\text { 5. } 2\end{array}$ \\
\hline \multicolumn{2}{|c|}{$\begin{array}{l}11 \\
12 \\
13 \\
15\end{array}$} & $\begin{array}{r}15.1 \\
7.2 \\
5.4 \\
7.3 \\
4.8\end{array}$ & $\begin{array}{l}9.0 \\
6.8 \\
6.4 \\
5.9 \\
\text { 5. }\end{array}$ & $\begin{array}{r}16.4 \\
5.9 \\
4.8 \\
4.5 \\
4.2\end{array}$ & $\begin{array}{l}3.7 \\
4.0 \\
3.9 \\
5.1 \\
\text { 5. } 2\end{array}$ & $\begin{array}{r}18.0 \\
6.6 \\
6.4 \\
19.6 \\
9.2\end{array}$ & $\begin{array}{l}15.4 \\
11.7 \\
19.5 \\
23 \\
14.0\end{array}$ & $\begin{array}{r}6.1 \\
5.5 \\
5.4 \\
17.2 \\
27\end{array}$ & $\begin{array}{r}5.2 \\
5.6 \\
5.1 \\
8.9 \\
14.5\end{array}$ & $\begin{array}{r}8.9 \\
8.1 \\
6.9 \\
\times 6.4 \\
65.9\end{array}$ & $\begin{array}{r}10.6 \\
9.5 \\
8.8 \\
\cdot 5.9 \\
7.6\end{array}$ & $\begin{array}{r}6.4 \\
5.2 \\
10.3 \\
7.8 \\
5.5\end{array}$ & $\begin{array}{l}\text { 5. } 2 \\
5.5 \\
7.7 \\
9.4\end{array}$ \\
\hline \multicolumn{2}{|c|}{$\begin{array}{l}16 \\
17 \\
18 \\
20\end{array}$} & $\begin{array}{r}6.9 \\
12.0 \\
5.7 \\
5.2 \\
4.3\end{array}$ & $\begin{array}{r}6.2 \\
13.5 \\
9.0 \\
5.4 \\
8.9\end{array}$ & $\begin{array}{r}4.8 \\
5.2 \\
5.8 \\
15.0 \\
5.0\end{array}$ & $\begin{array}{l}3.9 \\
3.7 \\
3.7 \\
3.5 \\
3.4\end{array}$ & $\begin{array}{r}6.4 \\
5.7 \\
8.1 \\
8.5 \\
12.7\end{array}$ & $\begin{array}{r}12.8 \\
8.5 \\
7.0 \\
6.4 \\
5.9\end{array}$ & $\begin{array}{l}a 16.4 \\
810.1 \\
a 7.2 \\
13.8 \\
82\end{array}$ & $\begin{array}{l}25 \\
79 \\
24 \\
56 \\
27\end{array}$ & $\begin{array}{r}0.8 \\
6.5 \\
5.3 \\
5.6 \\
5.3\end{array}$ & $\begin{array}{r}5.6 \\
5.5 \\
21 \\
8.8 \\
5.9\end{array}$ & $\begin{array}{l}4.6 \\
4.5 \\
4.4 \\
4.3 \\
4.3\end{array}$ & $\begin{array}{l}5 . \\
4 . \\
4 \\
4 . \\
4 .\end{array}$ \\
\hline \multicolumn{2}{|c|}{ 21 24} & \begin{tabular}{r|}
4.3 \\
4.2 \\
4.2 \\
6.0 \\
26
\end{tabular} & $\begin{array}{l}5.7 \\
5.0 \\
4.5 \\
6.6 \\
5.2\end{array}$ & $\begin{array}{l}4.3 \\
4.2 \\
4.0 \\
3.9 \\
3.7\end{array}$ & $\begin{array}{l}3.4 \\
3.5 \\
3.5 \\
3.7 \\
3.4\end{array}$ & $\begin{array}{l}8.6 \\
5.4 \\
4.3 \\
4.3 \\
3.6\end{array}$ & $\begin{array}{r}6.1 \\
6.8 \\
5.7 \\
6.1 \\
51\end{array}$ & $\begin{array}{c}43 \\
227 \\
34 \\
12.5 \\
9.2\end{array}$ & $\begin{array}{l}23 \\
25 \\
12.5 \\
9.8 \\
7.8\end{array}$ & $\begin{array}{l}5.1 \\
5.1 \\
4.8 \\
5.5 \\
5.9\end{array}$ & $\begin{array}{l}5.3 \\
4.8 \\
4.8 \\
5.5 \\
5.2\end{array}$ & $\begin{array}{l}4.3 \\
4.4 \\
4.6 \\
4.6 \\
4.8\end{array}$ & $\begin{array}{r}4.3 \\
4.4 \\
4.3 \\
5.7 \\
16.5\end{array}$ \\
\hline \multicolumn{2}{|c|}{$\begin{array}{l}26 \\
28 \\
20 \\
31\end{array}$} & $\begin{aligned} 47 \\
63 \\
13.6 \\
8.2 \\
6.4 \\
5.7\end{aligned}$ & $\begin{array}{l}5.0 \\
4.5 \\
4.5 \\
4.5 \\
3.9 \\
3.7\end{array}$ & \begin{tabular}{l|}
3.7 \\
3.6 \\
4.7 \\
4.2 \\
3.7
\end{tabular} & $\begin{array}{l}\text { 3.4 } \\
4.3 \\
5.2 \\
3.7 \\
\text { 3. } \\
3.4\end{array}$ & $\begin{array}{l}\text { 3.4 } \\
\text { 3.2 } \\
\text { 3.1 } \\
\text { 3.0 } \\
\text { 3.0 }\end{array}$ & $\begin{array}{r}12.6 \\
7.7 \\
11.9 \\
8.4 \\
6.4 \\
6.6\end{array}$ & $\begin{array}{r}8.6 \\
8.9 \\
12.1 \\
7.3 \\
6.4 \\
5.8\end{array}$ & $\begin{array}{r}11.8 \\
8.3 \\
9.2 \\
-2\end{array}$ & $\begin{array}{l}5.6 \\
4.9 \\
4.9 \\
4.8 \\
4.6 \\
4.6\end{array}$ & $\begin{array}{l}4.5 \\
4.5 \\
4.4 \\
4.4 \\
4.3\end{array}$ & $\begin{array}{l}6.2 \\
5.6 \\
7.5 \\
5.2 \\
4.8 \\
4.6\end{array}$ & $\begin{array}{r}6.7 \\
5.2 \\
4.6 \\
4.5 \\
4.4 \\
\\
\end{array}$ \\
\hline Day & July & Aug. & Sept & & $y$ & July & Aug. & Sept. & & ay & July & Aug. & Sept. \\
\hline $\begin{array}{c}1929 \\
3 \\
3 \\
5 \\
\end{array}$ & $\begin{array}{l}4.4 \\
4.4 \\
4.1 \\
4.0 \\
4.0\end{array}$ & $\begin{array}{l}5.0 \\
4.8 \\
7.8 \\
7.0 \\
\text { 5. } 6\end{array}$ & $\begin{array}{l}4.8 \\
4.6 \\
4.5 \\
4.4 \\
4.3\end{array}$ & $\begin{array}{l}11 . \\
12 . \\
13 . \\
14 . \\
15 .\end{array}$ & 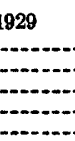 & $\begin{array}{l}4.0 \\
3.9 \\
4.0 \\
3.9 \\
5.1\end{array}$ & $\begin{array}{r}17.3 \\
6.6 \\
5.6 \\
5.2 \\
5.1\end{array}$ & ים & $\begin{array}{l}21 . \\
22 . \\
23 . \\
24 . \\
25 .\end{array}$ & 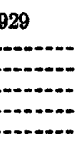 & $\begin{array}{l}4.4 \\
4.4 \\
4.2 \\
4.5 \\
7.1\end{array}$ & $\begin{array}{r}5.3 \\
4.9 \\
4.8 \\
4.8 \\
10.9\end{array}$ & - \\
\hline $\begin{array}{r}6 \\
8 \\
8 \\
8\end{array}$ & $\begin{array}{l}4.0 \\
4.1 \\
4.1 \\
4.0 \\
4.0\end{array}$ & $\begin{array}{r}5.3 \\
4.9 \\
5.1 \\
6.2 \\
23\end{array}$ & $\mid$\begin{tabular}{l}
$-\ldots-$ \\
\hdashline$-\cdots$ \\
\hdashline$-\cdots$ \\
\hdashline$-\cdots$
\end{tabular} & & - & $\begin{array}{l}23 \\
5.3 \\
4.5 \\
4.3 \\
4.2\end{array}$ & $\begin{array}{l}5.2 \\
4.8 \\
4.8 \\
5.3 \\
\text { 5. } 3\end{array}$ & - & $\begin{array}{l}26 \\
27 . \\
28 . \\
29 \\
30 \\
31 .\end{array}$ & $\ldots$ & $\begin{array}{l}22 \\
10.5 \\
5.9 \\
5.1 \\
4.6 \\
4.8\end{array}$ & $\begin{array}{l}6.4 \\
5.2 \\
4.9 \\
4.8 \\
5.1 \\
4.6\end{array}$ & - \\
\hline
\end{tabular}

a Partly estimated.

Bstimated. 
ISLAND OF MOLOKAI

Monthly discharge of Lanipuni Stream near Pelekunu, Molokai, 19\$8-\$9

\begin{tabular}{|c|c|c|c|c|c|c|}
\hline \multirow{2}{*}{ Month } & \multicolumn{3}{|c|}{ Million gallons a day } & \multirow{2}{*}{$\begin{array}{l}\text { Second- } \\
\text { foet } \\
\text { (mean) }\end{array}$} & \multicolumn{2}{|c|}{ Total run-ofi } \\
\hline & Maximum & Minimum & Mean & & $\begin{array}{l}\text { Million } \\
\text { gallons }\end{array}$ & Acre-feet \\
\hline $\begin{array}{l}\text { July } \\
\text { August } \\
\text { September } \\
\text { October } \\
\text { November } \\
\text { Docember } \\
\text { January } \\
\text { February } \\
\text { March } \\
\text { April } \\
\text { May } \\
\text { June }\end{array}$ & $\begin{array}{l}63 \\
13.5 \\
34 \\
12.4 \\
47 \\
131 \\
227 \\
79 \\
61 \\
27 \\
10.3 \\
16.5\end{array}$ & $\begin{array}{l}4.2 \\
\text { 3. } \\
3.6 \\
3.4 \\
3.0 \\
2.7 \\
5.2 \\
5.1 \\
4.6 \\
4.3 \\
4.2 \\
4.3\end{array}$ & $\begin{array}{r}12.3 \\
6.28 \\
6.35 \\
4.45 \\
8.25 \\
17.7 \\
21.2 \\
14.7 \\
12.1 \\
6.95 \\
5.30 \\
5.66\end{array}$ & $\begin{array}{l}19.0 \\
9.72 \\
9.82 \\
6.89 \\
12.8 \\
27.4 \\
32.8 \\
22.7 \\
18.7 \\
10.8 \\
8.20 \\
8.76\end{array}$ & $\begin{array}{l}381 \\
195 \\
190 \\
138 \\
248 \\
548 \\
659 \\
413 \\
374 \\
208 \\
164 \\
170\end{array}$ & $\begin{array}{r}1,170 \\
597 \\
585 \\
423 \\
760 \\
1,680 \\
2,020 \\
1,260 \\
1,150 \\
640 \\
504 \\
521\end{array}$ \\
\hline The year. & 227 & 2.7 & 10.1 & 15.6 & 3,690 & 11,300 \\
\hline $\begin{array}{l}\text { July } 1929 \\
\text { August } \\
\text { Beptember (5 days) }\end{array}$ & $\begin{array}{l}23 \\
23 \\
4.8\end{array}$ & $\begin{array}{l}3.9 \\
4.6 \\
4.3\end{array}$ & $\begin{array}{l}5.83 \\
6.50 \\
4.52\end{array}$ & $\begin{array}{c}9.02 \\
10.1 \\
6.99\end{array}$ & $\begin{array}{l}181 \\
202 \\
22.6\end{array}$ & $\begin{array}{r}555 \\
618 \\
69\end{array}$ \\
\hline
\end{tabular}


WAIKOLU STREAM AT PIPE-IINE CBOSSING NEAR KALAUPAPA, MOLOKAT

Location.-Water-stage recorder at elevation 300 feet, 1 mile above mouth of stream, and 4 miles southeast of Kalaupapa.

Drainage area. - 3.7 square miles.

Records aVaILABLe.-June, 1919, to June, 1929.

EXTREmas.- Maximum discharge during year, 772 million gallons a day or 1,190 second-feet Jan. 20 (gage height, 5.05 feet); minimum, 3.2 million gallons a day or 5.0 second-feet July 27 .

1919-1929: Maximum discharge, 1,270 million gallons a day or 1,960 second-feet Dec. 24, 1920 (gage height, 10.20 feet); minimum, 1.3 million gallons a day or 2.0 second-feet Nov. 1-2, 1925, June 5, 1926.

REMARKs. - Records fair for ordinary stages; poor for estimated periods and extremely high stages. Kalaupapa water-supply system diverts above station for domestic use.

Discharge, in million gallons a day, 1928-29

\begin{tabular}{|c|c|c|c|c|c|c|c|c|c|c|c|c|}
\hline Day & July & Aug. & Sept. & Oct. & Nov. & Dec. & Jan. & Feb. & Mar. & Apr. & May & June \\
\hline $\begin{array}{l}2 \\
3 \\
4 \\
5\end{array}$ & $\begin{array}{l}23 \\
8.4 \\
8.4 \\
6.8 \\
6.8\end{array}$ & $\begin{array}{l}6.5 \\
6.5 \\
6.2 \\
6.2 \\
6.2\end{array}$ & $\begin{array}{l}6.2 \\
6.2 \\
6.2 \\
6.9 \\
6.6\end{array}$ & $\begin{array}{l}6.2 \\
6.6 \\
5.5 \\
5.5 \\
8.2\end{array}$ & $\begin{array}{l}6.0 \\
5.7 \\
5.5 \\
6.9 \\
9.8\end{array}$ & $\begin{array}{r}4.8 \\
4.8 \\
4,8 \\
4.8 \\
99\end{array}$ & 6 & $\begin{array}{l}6.6 \\
6.6 \\
6.6 \\
6.6 \\
6.6\end{array}$ & $\begin{array}{l}69.5 \\
16.8 \\
17.3 \\
48 \\
63\end{array}$ & $\begin{array}{l}5.7 \\
5.7 \\
5.7 \\
5.7 \\
5.7\end{array}$ & $\begin{array}{r}5.5 \\
5.5 \\
5.5 \\
a 5.5\end{array}$ & \\
\hline 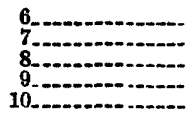 & 15.8 & $\begin{array}{r}5.9 \\
6.8 \\
6.2 \\
5.9 \\
14.3\end{array}$ & $\begin{array}{r}6.6 \\
6.6 \\
6.6 \\
6.2 \\
12.3\end{array}$ & $\begin{array}{l}6.2 \\
5.7 \\
5.0 \\
4.8 \\
4.8\end{array}$ & $\begin{array}{r}9.4 \\
48 \\
12.2 \\
12.7 \\
7.6\end{array}$ & $\begin{array}{l}36 \\
8.4 \\
11.7 \\
48 \\
17.0\end{array}$ & $\begin{array}{r}\text { b } 30 \\
6.6 \\
\mathbf{5 . 5}\end{array}$ & $\begin{array}{l}6.6 \\
6.9 \\
6.9 \\
6.6 \\
6.2\end{array}$ & $\begin{array}{l}25 \\
23 \\
17.1 \\
12.7 \\
9.4\end{array}$ & $\begin{array}{r}5.7 \\
5.5 \\
5.7 \\
5.7 \\
25\end{array}$ & & \\
\hline 11 12 & $\begin{array}{r}10.9 \\
6.5 \\
6.2 \\
10.5 \\
6.5\end{array}$ & $\begin{array}{l}9.7 \\
6.8 \\
6.5 \\
6.2 \\
6.2\end{array}$ & $\begin{array}{r}\mathbf{1 7 . 0} \\
7.3 \\
\mathbf{6 . 2} \\
\mathbf{6 . 0} \\
\mathbf{5 . 7}\end{array}$ & $\begin{array}{l}4.6 \\
4.6 \\
4.8 \\
5.0 \\
4.8\end{array}$ & $\begin{array}{l}35 \\
8.7 \\
8.0 \\
21 \\
13.2\end{array}$ & $\begin{array}{r}11.0 \\
8.0 \\
10.2 \\
9.4 \\
8.7\end{array}$ & $\begin{array}{l}5,3 \\
4.8 \\
4.8 \\
26 \\
40\end{array}$ & $\begin{array}{r}6.2 \\
7.6 \\
6.9 \\
6.9 \\
24\end{array}$ & $\begin{array}{l}8.4 \\
8.0 \\
8.0 \\
8.0 \\
8.4\end{array}$ & $\begin{array}{r}19.9 \\
7.3 \\
8.7 \\
6.2 \\
6.2\end{array}$ & & 6 \\
\hline 16 18 & $\begin{array}{r}7.6 \\
13.4 \\
7.2 \\
6.5 \\
6.2\end{array}$ & $\begin{array}{r}5.9 \\
7.4 \\
11.3 \\
6.6 \\
6.9\end{array}$ & $\begin{array}{l}5.7 \\
6.0 \\
6.6 \\
9.1 \\
7.3\end{array}$ & $\begin{array}{l}4.8 \\
4.8 \\
4.8 \\
4.6 \\
4.6\end{array}$ & $\begin{array}{r}7.6 \\
6.9 \\
9.1 \\
9.4 \\
22\end{array}$ & $\begin{array}{l}9.1 \\
6.6 \\
5.7 \\
5.5 \\
5.5\end{array}$ & $\begin{array}{l}14.5 \\
9.2 \\
6.9 \\
24 \\
83\end{array}$ & $\begin{array}{l}29 \\
74 \\
26 \\
63 \\
33\end{array}$ & $\begin{array}{l}8.0 \\
8.0 \\
8.0 \\
8.4 \\
8.4\end{array}$ & $\begin{array}{r}6.0 \\
6.0 \\
16.9 \\
11.8 \\
6.6\end{array}$ & 6 & \\
\hline 221 & $\begin{array}{r}6.2 \\
5.9 \\
5.9 \\
5.9 \\
10.6\end{array}$ & $\begin{array}{l}7.6 \\
6.2 \\
6.2 \\
6.2 \\
6.6\end{array}$ & $\begin{array}{l}6.0 \\
5.5 \\
5.5 \\
6.0 \\
6.2\end{array}$ & $\begin{array}{l}4.6 \\
4.6 \\
4.8 \\
4.8 \\
4.8\end{array}$ & $\begin{array}{r}15.6 \\
6.2 \\
5.5 \\
5.5 \\
5.3\end{array}$ & $\begin{array}{r}5.5 \\
5.5 \\
5.3 \\
5.5 \\
46\end{array}$ & $\begin{array}{c}85 \\
220 \\
30 \\
11.0 \\
8.4\end{array}$ & $\begin{array}{l}35 \\
28 \\
9.4 \\
8.7 \\
7.3\end{array}$ & $\begin{array}{l}7.3 \\
6.6 \\
6.6 \\
6.6 \\
6.9\end{array}$ & $\begin{array}{l}6.2 \\
6.0 \\
6.0 \\
6.0 \\
6.2\end{array}$ & & $\begin{array}{r}\mathbf{6 . 7} \\
5.7 \\
6.0 \\
17.0\end{array}$ \\
\hline $\begin{array}{l}28 \\
27 \\
28 \\
30 \\
31\end{array}$ & $\begin{array}{l}42 \\
22 \\
9.7 \\
6.8 \\
6.2 \\
6.2\end{array}$ & $\begin{array}{l}6.2 \\
6.2 \\
6.2 \\
6.2 \\
6.0 \\
6.0\end{array}$ & $\begin{array}{l}6.2 \\
6.6 \\
6.6 \\
5.7 \\
5.3\end{array}$ & $\begin{array}{l}4.8 \\
5.0 \\
5.0 \\
5.7 \\
6.0 \\
5.5\end{array}$ & $\begin{array}{l}\mathbf{5 . 3} \\
\mathbf{5 . 0} \\
\mathbf{5 . 0} \\
\mathbf{5 . 0} \\
\mathbf{5 . 0}\end{array}$ & $\begin{array}{r}12.6 \\
6.2 \\
8\end{array}$ & $\begin{array}{r}7.6 \\
11.9 \\
32 \\
8.7 \\
7.3 \\
6.6\end{array}$ & 8 & $\begin{array}{l}6.6 \\
6.2 \\
6.0 \\
6.0 \\
5.7 \\
5.7\end{array}$ & $\begin{array}{l}6.0 \\
6.0 \\
6.0 \\
6.0 \\
5.7\end{array}$ & & $\begin{array}{l}9.0 \\
6.2 \\
5.7 \\
5.7 \\
5.7\end{array}$ \\
\hline
\end{tabular}

\begin{tabular}{|c|c|c|c|c|c|c|}
\hline \multirow{2}{*}{ Menth } & \multicolumn{3}{|c|}{ Million gallons a day } & \multirow{2}{*}{$\begin{array}{l}\text { Second- } \\
\text { feet } \\
\text { (mean) }\end{array}$} & \multicolumn{2}{|c|}{ Total run-off } \\
\hline & Maximum & um & Mean & & $\begin{array}{l}\text { Million } \\
\text { gallons }\end{array}$ & Acre-feet \\
\hline $\begin{array}{l}\text { July } \\
\text { August } \\
\text { September } \\
\text { October } \\
\text { November } \\
\text { December } \\
\text { January } \\
\text { February } \\
\text { March } \\
\text { April } \\
\text { May }\end{array}$ & \begin{tabular}{c}
42 \\
14.3 \\
17.0 \\
8.2 \\
48 \\
99 \\
220 \\
\hdashline 63 \\
25 \\
---
\end{tabular} & $\begin{array}{r}5.9 \\
5.3 \\
4.6 \\
5.0 \\
6.2 \\
5,7 \\
5.5 \\
\end{array}$ & $\begin{array}{c}10.1 \\
6.90 \\
6.90 \\
5.21 \\
10.9 \\
14.1 \\
23.6 \\
17.0 \\
12.7 \\
7.73 \\
5.94 \\
6.42\end{array}$ & $\begin{array}{c}15.6 \\
10.7 \\
10.7 \\
8.06 \\
16.9 \\
21.8 \\
36.5 \\
.26 .3 \\
19.6 \\
12.0 \\
9.19 \\
9.93\end{array}$ & $\begin{array}{l}314 \\
214 \\
207 \\
162 \\
328 \\
438 \\
731 \\
477 \\
394 \\
232 \\
184 \\
193\end{array}$ & $\begin{array}{r}961 \\
656 \\
635 \\
496 \\
1,000 \\
1,340 \\
2,250 \\
1,460 \\
1,210 \\
712 \\
565 \\
591\end{array}$ \\
\hline The year... & 220 & - & 10.6 & 16.4 & 3,870 & 11,900 \\
\hline
\end{tabular}




\section{MISCELLANEOUS MEASUREMHNTS}

Measurements of streams and ditches on the island of Molokai at other than regular gaging stations are listed below:

Miscellaneous discharge measurements on Molokai, 1928-29

\begin{tabular}{|c|c|c|c|c|c|}
\hline \multirow[b]{2}{*}{ Dato } & \multirow[b]{2}{*}{ Stream } & \multirow[b]{2}{*}{$\begin{array}{l}\text { Tributary to- } \\
\text { or diverting from }\end{array}$} & \multirow[b]{2}{*}{ Locality } & \multicolumn{2}{|c|}{ Discharge } \\
\hline & & & & $\begin{array}{c}\text { Second- } \\
\text { feet }\end{array}$ & $\begin{array}{l}\text { Million } \\
\text { gallons } \\
\text { a day }\end{array}$ \\
\hline Apr. & Waileia Stream. & Pacific Ocean & Elevation 2,750 feet, near & 0.113 & 0.073 \\
\hline & Waihanau Stream..... & ......do...... & Elevation 2,450 feet, near & .267 & .173 \\
\hline - & $\underset{\text { Commission tunnel. }}{\text { H aw alia n }}$ & Waihanau Stream. & Near Kalae. & .312 & .202 \\
\hline
\end{tabular}




\section{ISLAND OF MAUI}

\section{HONOKAHAU STREAM NEAR HONOKAHAO, MAUI}

LocAtion.-Water-stage recorder 1,000 feet above intake of Honokahau ditch and about 5 miles southeast of Honokahau; elevation 910 feet.

Drainage AREA. - 4.2 square miles.

Records available.-March, 1913, to September, 1920; May, 1922, to June, 1929.

Extremes.-1928-29; Not known, owing to loss of record.

1913-1920, 1922-1929: Maximum discharge, 2,200 million gallons a day or 3,400 second-feet Feb. 13, 1924 (gage height 7:92 feet); minimum, 6.2 million gallons a day or 9.6 second-feet June 30, 1926 .

REMARKs.- Records good except those for extremely high stages and estimated periods, which are poor. No diversions.

Discharge, in million gallons a day, 1928-29

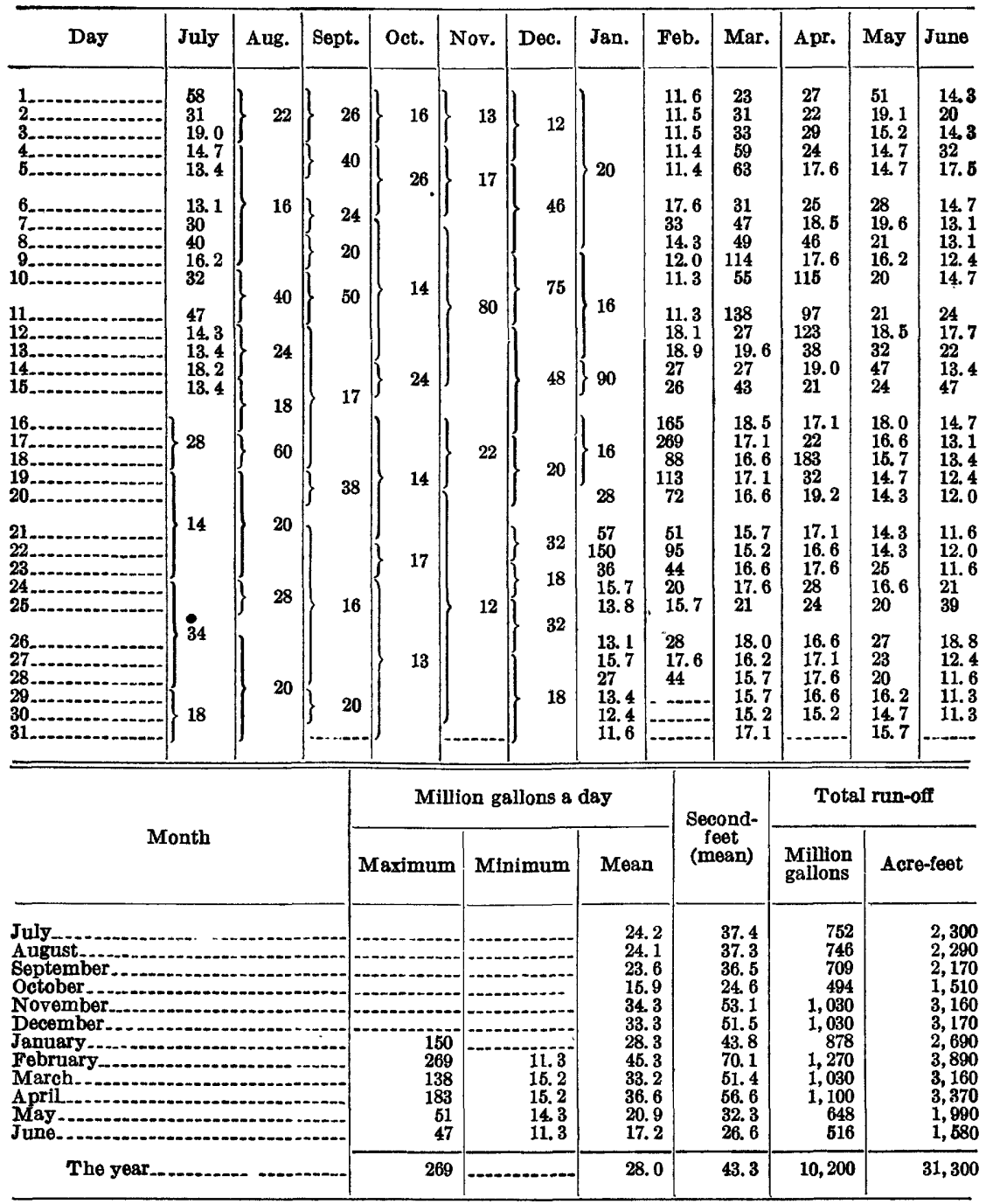




\section{HOMOKAWAI DITCH MEAR LAHAINA, MAUI}

LOCATION.-Water-stage recorder just below intake on Honokawai Stream, 21/2 miles above Pioneer Mill Co.'s power house and $7 \frac{1 / 2}{2}$ miles northeast of Lahaina.

RECORDS AVAir ABLE.-July, 1912, to June, 1929.

ExTREMEs.-Maximum discharge during year, 49 million gallons a day or 76 second-feet Nov. 7 (gage height, 2.00 feet); minimum, 3.3 million gallons a day or 5.1 second-feet July 6 .

1912-1929: Maximum discharge, 69 million gallons a day or 107 secondfeet Sept. 10, 1922 (gage height, 2.71 feet); no flow occasionally when water is shut out of ditch.

R EMARKs. - Records good. Diverts water for irrigation from Honokawai Stream just above station. Regulated by head gates at intake.

Discharge, in million gallons a day, 1928-29

\begin{tabular}{|c|c|c|c|c|c|c|c|c|c|c|c|c|}
\hline Day & July & Aug. & Sept. & Oct. I & Nov. & Dec. & Jan. & Feb. & Mar. & A pr. & May & June \\
\hline & $\begin{array}{l}7.6 \\
5.2 \\
4.2 \\
3.6\end{array}$ & $\begin{array}{r}7.2 \\
6.0 \\
15.2 \\
4.5 \\
4.9\end{array}$ & $\begin{array}{r}3.8 \\
10.5 \\
8.8 \\
13.2 \\
4.2\end{array}$ & \begin{tabular}{r|}
4.2 \\
4.0 \\
4.1 \\
7.7 \\
10.0
\end{tabular} & $\begin{array}{l}4.0 \\
4.0 \\
4.0 \\
4.6 \\
4.3\end{array}$ & $\begin{array}{r}4.1 \\
3.8 \\
3.8 \\
3.8 \\
22\end{array}$ & $\begin{array}{r}12.4 \\
6.3 \\
4.4 \\
4.2 \\
5.6\end{array}$ & $\begin{array}{l}4.0 \\
4.0 \\
4.0 \\
4.0 \\
4.0\end{array}$ & $\begin{array}{r}8.2 \\
11.9 \\
10.9 \\
12.1 \\
11.9\end{array}$ & $\begin{array}{l}4.1 \\
5.6 \\
4.4 \\
4.9 \\
4.1\end{array}$ & $\begin{array}{r}11.6 \\
5.1 \\
4.0 \\
4.0 \\
3.9\end{array}$ & $\begin{array}{r}4.1 \\
4.6 \\
4.8 \\
13.4 \\
5.6\end{array}$ \\
\hline$\cdots$ & \begin{tabular}{r|}
3.3 \\
9.2 \\
13.5 \\
4.2 \\
12.4
\end{tabular} & $\begin{array}{r}3.9 \\
4.9 \\
4.5 \\
3.9 \\
11.8\end{array}$ & $\begin{array}{r}3.9 \\
4.7 \\
4.8 \\
9.0 \\
26\end{array}$ & $\begin{array}{l}4.5 \\
4.4 \\
4.0 \\
4.0 \\
4.0\end{array}$ & $\begin{array}{r}5.0 \\
32 \\
10.4 \\
7.2 \\
8.9\end{array}$ & $\begin{array}{l}20 \\
7.0 \\
15.5 \\
26 \\
13.3\end{array}$ & $\begin{array}{r}7.4 \\
+5.6 \\
+8.3 \\
4.6 \\
4.2\end{array}$ & $\begin{array}{l}4.0 \\
6.8 \\
4.6 \\
4.0 \\
3.9\end{array}$ & $\begin{array}{r}7.8 \\
14.4 \\
13.4 \\
21 \\
10.7\end{array}$ & $\begin{array}{r}4.1 \\
4.4 \\
15.2 \\
4.2 \\
24\end{array}$ & $\begin{array}{l}5.0 \\
4.4 \\
4.6 \\
4.1 \\
4.8\end{array}$ & $\begin{array}{l}4.9 \\
4.5 \\
4.2 \\
4.2 \\
4.6\end{array}$ \\
\hline (n) & \begin{tabular}{r|r|}
11.4 \\
3.8 \\
3.6 \\
4.7 \\
3.6
\end{tabular} & $\begin{array}{r}9.7 \\
11.1 \\
12.8 \\
5.7 \\
5.2\end{array}$ & $\begin{array}{r}12.1 \\
4.8 \\
4.1 \\
4.0 \\
4.0\end{array}$ & $\begin{array}{l}4.0 \\
3.9 \\
3.9 \\
5.0 \\
6.5\end{array}$ & $\begin{array}{c}18.3 \\
10.7 \\
10.0 \\
27 \\
9.3\end{array}$ & $\begin{array}{l}10.8 \\
11.9 \\
14.2 \\
20 \\
13.7\end{array}$ & \begin{tabular}{r|}
4.1 \\
3.9 \\
4.0 \\
12.9 \\
14.2
\end{tabular} & \begin{tabular}{r|}
3.9 \\
7.6 \\
4.8 \\
13.4 \\
13.4
\end{tabular} & $\begin{array}{r}14.5 \\
5.8 \\
4.9 \\
4.6 \\
4.6\end{array}$ & \begin{tabular}{r|}
21 \\
35 \\
11.2 \\
4.8 \\
6.3
\end{tabular} & $\begin{array}{r}5.8 \\
4.8 \\
10.4 \\
11.7 \\
6.1\end{array}$ & $\begin{array}{r}9.8 \\
7.0 \\
8.6 \\
5.2 \\
16.8\end{array}$ \\
\hline ... & \begin{tabular}{r|}
15.9 \\
11.1 \\
5.2 \\
4.2 \\
3.6
\end{tabular} & $\begin{array}{r}13.2 \\
36 \\
13.0 \\
4.4 \\
8.1\end{array}$ & $\begin{array}{r}4.5 \\
4.4 \\
4.2 \\
21 \\
4.8\end{array}$ & $\begin{array}{l}4.1 \\
4.0 \\
4.0 \\
4.0 \\
4.0\end{array}$ & $\begin{array}{l}5.9 \\
5.0 \\
7.9 \\
6.6 \\
6.3\end{array}$ & $\begin{array}{r}17.4 \\
8.3 \\
7.0 \\
6.7 \\
5.8\end{array}$ & $\begin{array}{r}8.2 \\
4.6 \\
4.1 \\
14.1 \\
13.0\end{array}$ & $\begin{array}{l}27 \\
30 \\
15.6 \\
22 \\
20\end{array}$ & $\begin{array}{l}4.4 \\
4.2 \\
4.1 \\
4.1 \\
4.1\end{array}$ & $\begin{array}{r}4.1 \\
5.2 \\
28 \\
8.2 \\
4.5\end{array}$ & $\begin{array}{l}4.2 \\
4.2 \\
4.1 \\
4.1 \\
4.1\end{array}$ & $\begin{array}{l}5.5 \\
4.9 \\
4.4 \\
4.1 \\
4.1\end{array}$ \\
\hline (1) & \begin{tabular}{r|}
3.5 \\
6.6 \\
5.0 \\
17.2 \\
31
\end{tabular} & $\begin{array}{l}5.2 \\
4.5 \\
5.6 \\
9.5 \\
5.2\end{array}$ & $\begin{array}{l}4.2 \\
4.0 \\
4.0 \\
4.0 \\
4.0\end{array}$ & $\begin{array}{l}4.0 \\
5.4 \\
4.2 \\
4.0 \\
4.0\end{array}$ & $\begin{array}{l}5.0 \\
4.2 \\
4.2 \\
4.2 \\
4.2\end{array}$ & $\begin{array}{r}8.7 \\
5.9 \\
4.4 \\
6.2 \\
14.8\end{array}$ & \begin{tabular}{r|}
18.3 \\
19.1 \\
8.6 \\
5.4 \\
4.5
\end{tabular} & \begin{tabular}{r|}
19.2 \\
28 \\
11.3 \\
5.8 \\
4.6
\end{tabular} & $\begin{array}{l}4.1 \\
4.1 \\
4.0 \\
3.9 \\
3.9\end{array}$ & $\begin{array}{l}4.1 \\
4.0 \\
4.0 \\
6.6 \\
5.8\end{array}$ & $\begin{array}{l}4.1 \\
4.1 \\
7.1 \\
5.0 \\
8.0\end{array}$ & $\begin{array}{r}4.1 \\
4.1 \\
4.1 \\
5.8 \\
12.7\end{array}$ \\
\hline (1) & $\begin{array}{c}33 \\
16.9 \\
7.0 \\
4.9 \\
4.2 \\
5.5\end{array}$ & $\begin{array}{l}4.1 \\
4.1 \\
7.8 \\
3.9 \\
3.8 \\
3.6\end{array}$ & $\begin{array}{l}4.0 \\
4.0 \\
4.0 \\
4.9 \\
4.0\end{array}$ & $\begin{array}{l}4.0 \\
4.0 \\
4.0 \\
4.0 \\
4.1 \\
4.1\end{array}$ & $\begin{array}{l}4.2 \\
4.2 \\
4.3 \\
4.4 \\
4.3\end{array}$ & $\begin{array}{l}8.0 \\
4.8 \\
8.6 \\
7.3 \\
4.1 \\
4.0\end{array}$ & $\begin{array}{r}4.0 \\
4.5 \\
10.4 \\
4.4 \\
4.1 \\
4.1 \\
-\end{array}$ & $\begin{array}{r}4.6 \\
5.0 \\
16.1 \\
\\
\\
\end{array}$ & $\begin{array}{l}4.0 \\
3.9 \\
3.9 \\
3.9 \\
4.0 \\
4.0\end{array}$ & $\begin{array}{l}4.1 \\
4.2 \\
4.1 \\
4.1 \\
4.0\end{array}$ & $\begin{array}{r}10.1 \\
6.2 \\
6.1 \\
5.0 \\
4.4 \\
4.2\end{array}$ & $\begin{array}{l}6 . \\
4 . \\
4 . \\
4 . \\
4 \\
-\end{array}$ \\
\hline \multirow{2}{*}{\multicolumn{4}{|c|}{ Month }} & \multicolumn{4}{|c|}{ Million gallons a day } & \multirow{2}{*}{\multicolumn{2}{|c|}{$\begin{array}{l}\text { Second- } \\
\text { feet } \\
\text { (mean) }\end{array}$}} & \multicolumn{3}{|c|}{ Total run-ofl } \\
\hline & & & & Maximum & n Min & imum & Mean & & & $\begin{array}{l}\text { Million } \\
\text { gallons }\end{array}$ & Acre & 8-feet \\
\hline 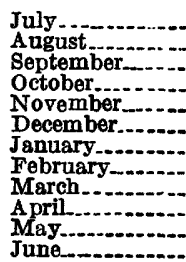 & - & & & $\begin{array}{l}33 \\
36 \\
26 \\
10.0 \\
32 \\
26 \\
19.1 \\
30 \\
21 \\
35 \\
11.7 \\
16.8\end{array}$ & \multicolumn{2}{|r|}{$\begin{array}{l}3.3 \\
3.6 \\
3.8 \\
3.9 \\
4.0 \\
3.8 \\
3.9 \\
3.9 \\
3.9 \\
4.0 \\
3.9 \\
4.1\end{array}$} & $\begin{array}{r}9.05 \\
7.85 \\
6.60 \\
4.52 \\
7.82 \\
10.1 \\
7.53 \\
10.6 \\
7.14 \\
8.28 \\
5.65 \\
5.98\end{array}$ & \multicolumn{2}{|c|}{$\begin{array}{c}14.0 \\
12.1 \\
10.2 \\
6.99 \\
12.1 \\
15.6 \\
11.7 \\
16.4 \\
11.0 \\
12.8 \\
8.74 \\
9.25\end{array}$} & $\begin{array}{l}198 \\
140 \\
235 \\
312 \\
234 \\
296 \\
221 \\
248 \\
175 \\
179\end{array}$ & & $\begin{array}{l}861 \\
747 \\
608 \\
430 \\
720 \\
961 \\
716 \\
911 \\
679 \\
762 \\
538 \\
551\end{array}$ \\
\hline \multicolumn{2}{|l|}{ The year.. } & 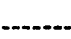 & & 36 & & 3.3 & 7.57 & & 11.7 & 2,760 & & 8,480 \\
\hline
\end{tabular}




\section{GANAHA STREAM ABOVE PIPE-IUNE INTAKE IEAR LAHATNA, MAUI}

Location.-Water-stage recorder 200 feet above intake of pipe line for Lahaina and Lahainaluna School and 23/4 miles northeast of Lahaina.

Drainage area.-1.8 square miles.

Reconds AVAMABLE.-February, 1916, to June, 1929.

ExTrmans.-Maximum discharge during year, 140 million gallons a day or 217 second-feet Feb. 17 (gage height, 3.43 feet); minimum, 2.8 million gallons a day or 4.3 second-feet frequently during first half of year.

1916-1929: Maximum discharge, 314 million gallons a day or 486 secondfeet Nov. 26, 1918 (gage height, 3.79 feet); minimum, 1.6 million gallons a day or 2.5 second-feet Dec. 23-25, 1927.

REMARKs.-Records good for ordinary stages; poor for high stages. No diversions

Discharge, in million gallons a day, 1928-29

\begin{tabular}{|c|c|c|c|c|c|c|c|c|c|c|c|c|}
\hline Day & July & Aug. & Sept. & Oct. & Nov. & Dec. & Jan. & Fob. & Mar. & Apr. & May & June \\
\hline $\begin{array}{l}1 \ldots \\
2 \ldots \\
3 \ldots \\
4 \ldots \\
6 \ldots\end{array}$ & $\begin{array}{l}4.6 \\
3.7 \\
2.9 \\
2.9\end{array}$ & $\begin{array}{l}3.7 \\
3.7 \\
5.7 \\
2.9 \\
2.8\end{array}$ & $\begin{array}{r}3.5 \\
5.0 \\
6.5 \\
10.0 \\
3.7\end{array}$ & $\begin{array}{l}2.9 \\
2.9 \\
2.9 \\
5.4 \\
6.3\end{array}$ & $\begin{array}{l}2.9 \\
2.9 \\
2.9 \\
4.2 \\
3.3\end{array}$ & $\begin{array}{r}2.8 \\
2.8 \\
a 2.8 \\
\times 2.9 \\
6.2\end{array}$ & $\begin{array}{l}5.4 \\
3.1 \\
3.1 \\
3.1 \\
4.9\end{array}$ & $\begin{array}{l}3.3 \\
\text { 3. } \\
\text { 3. } \\
\text { 3. } \\
\text { 3. } 3\end{array}$ & $\begin{array}{r}12.4 \\
10.4 \\
5.6 \\
6.3 \\
4.6\end{array}$ & $\begin{array}{l}4.3 \\
6.2 \\
4.6 \\
4.6 \\
4.3\end{array}$ & $\begin{array}{l}4.6 \\
3.5 \\
3.1 \\
3.1 \\
3.1\end{array}$ & $\begin{array}{r}13 . \\
6\end{array}$ \\
\hline $\begin{array}{r}6 \\
7-- \\
8-. \\
9-. \\
10 .-\end{array}$ & $\begin{array}{r}2.9 \\
10.1 \\
12.4 \\
6.0 \\
11.2\end{array}$ & $\begin{array}{l}2.8 \\
3.3 \\
3.1 \\
2.9 \\
9.6\end{array}$ & $\begin{array}{r}3.5 \\
5.0 \\
3.3 \\
4.3 \\
13.1\end{array}$ & $\begin{array}{l}3.3 \\
2.9 \\
2.9 \\
2.9 \\
2.9\end{array}$ & \begin{tabular}{r|r|}
3.5 & 18.7 \\
7.8 \\
7.8 \\
9.2
\end{tabular} & $\begin{array}{r}7.7 \\
4.5 \\
13.8 \\
17.3 \\
9.2\end{array}$ & $\begin{array}{l}4.6 \\
3.7 \\
4.5 \\
3.1 \\
3.1\end{array}$ & $\begin{array}{l}\text { 3. } \\
\text { 3.5 } \\
\text { 3. } 5 \\
\text { 3. } \\
\text { 3. }\end{array}$ & $\begin{array}{r}4.3 \\
10.1 \\
9.1 \\
10.3 \\
5.4\end{array}$ & $\begin{array}{r}4.3 \\
6.4 \\
11.6 \\
4.6 \\
9.6\end{array}$ & $\begin{array}{l}3.1 \\
3.1 \\
3.1 \\
3.3 \\
4.4\end{array}$ & \\
\hline 10 & $\begin{array}{l}5.7 \\
4.0 \\
3.5 \\
7.0 \\
4.9\end{array}$ & $\begin{array}{l}\mathbf{6 . 2} \\
\mathbf{4 . 3} \\
\mathbf{6 . 0} \\
\mathbf{5 . 2} \\
\mathbf{3 . 3}\end{array}$ & $\begin{array}{l}8.0 \\
3.3 \\
2.9 \\
2.9 \\
2.9\end{array}$ & $\begin{array}{l}2.9 \\
3.1 \\
3.1 \\
6.1 \\
5.5\end{array}$ & $\begin{array}{r}17.4 \\
7.2 \\
8.3 \\
11.7 \\
5.2\end{array}$ & $\begin{array}{r}8.3 \\
6.6 \\
6.5 \\
13.4 \\
7.2\end{array}$ & $\begin{array}{l}2.9 \\
2.9 \\
2.9 \\
9.3 \\
7.5\end{array}$ & $\begin{array}{r}3.3 \\
6.0 \\
3.5 \\
5.9 \\
11.0\end{array}$ & $\begin{array}{l}8.0 \\
4.3 \\
3.7 \\
3.7 \\
4.0\end{array}$ & $\begin{array}{r}7.5 \\
21 \\
8.1 \\
4.9 \\
7.6\end{array}$ & $\begin{array}{l}\mathbf{5 . 1} \\
\mathbf{5 . 3} \\
9.1 \\
6.0 \\
4.0\end{array}$ & $\begin{array}{r}10 . \\
5 . \\
12 .\end{array}$ \\
\hline 18 & $\begin{array}{r}10.9 \\
6.5 \\
4.0 \\
3.1 \\
3.1\end{array}$ & $\begin{array}{r}3.8 \\
12.8 \\
6.7 \\
3.3 \\
3.7\end{array}$ & $\begin{array}{l}3.1 \\
2.9 \\
2.8 \\
7.8 \\
3.1\end{array}$ & $\begin{array}{l}3.3 \\
\text { 3.1 } \\
\text { 3.1 } \\
\text { 3.1 } \\
\text { 3.1 }\end{array}$ & $\begin{array}{l}3.3 \\
3.8 \\
4.8 \\
3.6 \\
2.9\end{array}$ & $\begin{array}{l}7.0 \\
4.6 \\
4.0 \\
4.4 \\
4.3\end{array}$ & $\begin{array}{l}6.3 \\
3.3 \\
3.3 \\
6.7 \\
7.9\end{array}$ & $\begin{array}{l}29 \\
34 \\
30.6 \\
7.8 \\
13.6\end{array}$ & $\begin{array}{l}3.7 \\
3.7 \\
3.5 \\
3.5 \\
\mathbf{3 . 5}\end{array}$ & $\begin{array}{r}4.0 \\
4.3 \\
12.6 \\
4.3 \\
3.3\end{array}$ & $\begin{array}{l}3.7 \\
3.7 \\
3.7 \\
3.7 \\
3.7\end{array}$ & \\
\hline 2124 & $\begin{array}{r}3.1 \\
3.1 \\
3.7 \\
7.1 \\
11.2\end{array}$ & $\begin{array}{l}3.5 \\
3.3 \\
4.0 \\
6.1 \\
4.2\end{array}$ & $\begin{array}{l}3.1 \\
2.9 \\
2.9 \\
2.9 \\
2.9\end{array}$ & $\begin{array}{l}3.1 \\
4.1 \\
\text { 3.1 } \\
\text { 3. } \\
2.9\end{array}$ & $\begin{array}{l}2.8 \\
2.8 \\
2.8 \\
2.8 \\
2.8\end{array}$ & $\begin{array}{l}5.4 \\
5.6 \\
3.7 \\
3.7 \\
6.2\end{array}$ & $\begin{array}{l}9.8 \\
7.0 \\
4.8 \\
3.5 \\
3.5\end{array}$ & $\begin{array}{r}14.9 \\
21 \\
5.7 \\
4.0 \\
3.7\end{array}$ & $\begin{array}{l}\mathbf{3 . 5} \\
\mathbf{3 . 7} \\
\mathbf{3 . 7} \\
\mathbf{3 . 7} \\
4.0\end{array}$ & $\begin{array}{l}3.3 \\
3.3 \\
3.3 \\
4.8 \\
3.3\end{array}$ & $\begin{array}{l}3.7 \\
4.0 \\
8.2 \\
4.9 \\
\text { 5. }\end{array}$ & $\begin{array}{c}6 . \\
10 .\end{array}$ \\
\hline $\begin{array}{l}26 \\
27 \\
28 \\
30 \\
31\end{array}$ & $\begin{array}{r}10.4 \\
8.5 \\
3.5 \\
2.9 \\
2.9 \\
3.9\end{array}$ & $\begin{array}{l}4.0 \\
4.0 \\
4.6 \\
3.5 \\
3.3 \\
3.1\end{array}$ & $\begin{array}{l}2.9 \\
2.9 \\
3.3 \\
4.8 \\
2.9\end{array}$ & $\begin{array}{l}2.9 \\
2.9 \\
2.9 \\
2.9 \\
3.1 \\
2.9\end{array}$ & $\begin{array}{l}2.8 \\
2.8 \\
2.8 \\
2.8 \\
2.8\end{array}$ & $\begin{array}{l}5.2 \\
3.9 \\
4 . \\
4 . \\
3 .\end{array}$ & $\begin{array}{l}3.5 \\
3.7 \\
5.9 \\
3.5 \\
3.3 \\
3.3\end{array}$ & $\begin{array}{r}3.5 \\
3.8 \\
12.3\end{array}$ & $\begin{array}{l}4.0 \\
4.0 \\
4.3 \\
4.3 \\
4.3 \\
4.3\end{array}$ & $\begin{array}{l}3.3 \\
4.0 \\
3.3 \\
3.3 \\
3.1\end{array}$ & $\begin{array}{l}6.8 \\
5.8 \\
6.7 \\
4.0\end{array}$ & 4 \\
\hline
\end{tabular}

\begin{tabular}{|c|c|c|c|c|c|c|}
\hline \multirow{2}{*}{ Month } & \multicolumn{3}{|c|}{ Million gallons a day } & \multirow{2}{*}{$\begin{array}{l}\text { Second- } \\
\text { feet } \\
\text { (mean) }\end{array}$} & \multicolumn{2}{|c|}{ Total run-off } \\
\hline & Maximum & Minimum & Mean & & $\begin{array}{l}\text { Million } \\
\text { gallons }\end{array}$ & Acre-feet \\
\hline 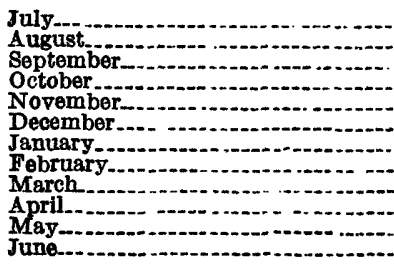 & $\begin{array}{l}12.4 \\
12.8 \\
13.1 \\
6.3 \\
18.7 \\
17.3 \\
9.8 \\
34 \\
12.4 \\
21 \\
9.1 \\
13.5\end{array}$ & \begin{tabular}{l|}
2.9 \\
2.8 \\
2.8 \\
2.9 \\
2.8 \\
2.8 \\
2.9 \\
3.3 \\
3.5 \\
3.1 \\
3.1 \\
3.3
\end{tabular} & $\begin{array}{l}5.72 \\
4.46 \\
4.30 \\
3.41 \\
5.31 \\
5.93 \\
4.63 \\
8.10 \\
5.29 \\
5.77 \\
4.45 \\
5.12\end{array}$ & $\begin{array}{l}8.85 \\
6.90 \\
6.65 \\
5.28 \\
8.22 \\
9.18 \\
7.16 \\
12.5 \\
8.18 \\
8.93 \\
6.89 \\
7.92\end{array}$ & $\begin{array}{l}177 \\
138 \\
129 \\
106 \\
159 \\
184 \\
113 \\
227 \\
164 \\
173 \\
138 \\
154\end{array}$ & $\begin{array}{l}544 \\
424 \\
396 \\
324 \\
489 \\
564 \\
440 \\
696 \\
503 \\
531 \\
423 \\
471\end{array}$ \\
\hline The year & 34 & 2.8 & 5. 18 & $\overline{8.01}$ & 1,890 & 5,800 \\
\hline
\end{tabular}

a Partly estimated. 


\section{OLOWAIU DITOH NEAR OLOWAIJ, MAUI}

LOCATION.-Water-stage recorder 425 feet above intake to penstock of hydroelectric power station, 1 mile above Olowalu, and 7 miles east of Lahaina.

ReCords AVAIL aBLE.- August, 1911, to June, 1929.

Extremes.-Maximum discharge during year, 8.6 million gallons a day or 13.3 second-feet Dec. 26 (gage height, 1.18 feet); minimum, 1.4 million gallons a day or 2.2 second-feet Apr. 4.

1912-1929: Maximum discharge, 18 million gallons a day or 28 secondfeet Dec. 25, 1920 (gage height, 1.53 feet); no flow occasionally when water is shut out of ditch.

REMARKs.- - Records good except those for estimated periods, which are poor. Intake in Olowalu Stream at about 450 feet elevation. Water used for power and irrigation. Regulated by head gates.

Discharge, in million gallons a day, 1928-29

\begin{tabular}{|c|c|c|c|c|c|c|c|c|c|c|c|c|}
\hline D & July & ug. & Sept. & Oct. & Nov. & Dec. & Jan. & Feb. & Mar. & Apr. & May & Jun \\
\hline $\begin{array}{l}1 \ldots \\
2 \ldots \\
3 \ldots \\
4 \ldots \\
5 \ldots\end{array}$ & $\begin{array}{l}8.0 \\
8.0 \\
7.3 \\
5.6 \\
4.3\end{array}$ & 3.8 & $\begin{array}{l}4.9 \\
5.9 \\
6.8 \\
7.3 \\
\text { 5.9 }\end{array}$ & $\begin{array}{r}\cdot 3.9 \\
3.9 \\
\cdot 4.0\end{array}$ & $\begin{array}{l}3.5 \\
3.2 \\
2.9 \\
3.4 \\
3.6\end{array}$ & $\begin{array}{l}\text { 3. } 0 \\
\text { 3. } 0 \\
\text { 3. } 2 \\
\text { 3. } 0 \\
\text { 5. } 1\end{array}$ & $\begin{array}{l}6.6 \\
5.0 \\
4.7 \\
4.4 \\
4.7\end{array}$ & $\begin{array}{l}4.4 \\
4.1 \\
3.8 \\
3.8 \\
3.8\end{array}$ & $\begin{array}{l}7.0 \\
7.2 \\
7.3 \\
7.6 \\
7.3\end{array}$ & $\begin{array}{l}4.7 \\
4.4 \\
4.1 \\
3.8 \\
4.0\end{array}$ & $\begin{array}{l}4.3 \\
4.0 \\
3.8 \\
3.6 \\
3.6\end{array}$ & \\
\hline $\begin{array}{c}6 \ldots \\
7 \ldots \\
8 \ldots \\
9 \\
10 \\
\ldots\end{array}$ & $\begin{array}{l}3.9 \\
4.8 \\
6.1 \\
4.6 \\
4.5\end{array}$ & & $\begin{array}{l}5.2 \\
5.7 \\
5.2 \\
5.4 \\
5.8\end{array}$ & 3.8 & $\begin{array}{l}2.5 \\
6.8 \\
6.3 \\
5.2 \\
4.6\end{array}$ & $\begin{array}{l}7.3 \\
5.0 \\
5.5 \\
8.4 \\
8.4\end{array}$ & $\begin{array}{l}5.3 \\
5.4 \\
6.2 \\
4.7 \\
4.1\end{array}$ & $\begin{array}{l}4.2 \\
5.0 \\
4.1 \\
3.8 \\
3.5\end{array}$ & $\begin{array}{l}7.2 \\
7.6 \\
7.6 \\
8.0 \\
8.0\end{array}$ & $\begin{array}{l}4.3 \\
4.4 \\
4.9 \\
4.3 \\
7.6\end{array}$ & $\begin{array}{l}3.8 \\
3.6 \\
3.7 \\
3.5 \\
3.8\end{array}$ & \\
\hline L. & $\begin{array}{l}5.3 \\
4.0 \\
\text { 3. } 6 \\
3.6 \\
3.2\end{array}$ & 6.5 & $\begin{array}{l}7.3 \\
7.3 \\
7.0 \\
6.6 \\
6.8\end{array}$ & $\begin{array}{l}3.3 \\
3.2 \\
3.3 \\
3.6\end{array}$ & $\begin{array}{l}7.6 \\
7.3 \\
7.3 \\
7.3 \\
7.6\end{array}$ & $\begin{array}{l}7.7 \\
6.6 \\
6.6 \\
8.4 \\
8.4\end{array}$ & $\begin{array}{l}3.8 \\
3.8 \\
3.8 \\
6.7 \\
7.3\end{array}$ & $\begin{array}{l}3.4 \\
3.5 \\
3.4 \\
3.4 \\
3.9\end{array}$ & $\begin{array}{l}8.0 \\
7.6 \\
7.0 \\
6.6 \\
7.3\end{array}$ & $\begin{array}{l}8.0 \\
8.4 \\
8.4 \\
6.6 \\
5.6\end{array}$ & $\begin{array}{l}4.0 \\
3.7 \\
4.6 \\
5.6 \\
5.2\end{array}$ & \\
\hline $\begin{array}{l}18 . \\
19 .\end{array}$ & $\begin{array}{l}6.0 \\
7.6 \\
7.0 \\
6.6 \\
4.9\end{array}$ & 7.5 & $\begin{array}{l}5.6 \\
5.2 \\
4.9 \\
6.8 \\
6.8\end{array}$ & $\begin{array}{l}\text { 3. } 1 \\
\text { 3. } 1 \\
\text { 3. } 0 \\
\text { 3. } 0 \\
\text { 2. } 8\end{array}$ & $\begin{array}{l}7.3 \\
5.9 \\
5.0 \\
4.7 \\
4.7\end{array}$ & $\begin{array}{l}8.4 \\
8.4 \\
8.4 \\
8.0 \\
7.3\end{array}$ & $\begin{array}{l}7.6 \\
5.6 \\
6.0 \\
5.8 \\
6.6\end{array}$ & $\begin{array}{l}5.9 \\
5.8 \\
6.4 \\
7.6 \\
7.6\end{array}$ & $\begin{array}{l}7.0 \\
6.6 \\
6.2 \\
6.2 \\
5.9\end{array}$ & $\begin{array}{l}4.9 \\
4.9 \\
8.3 \\
8.7 \\
8.0\end{array}$ & $\begin{array}{l}4.6 \\
4.3 \\
4.0 \\
3.8 \\
3.6\end{array}$ & \\
\hline $\begin{array}{l}21 \ldots \\
22-\ldots \\
23 \ldots \\
24 \ldots \\
25 \ldots\end{array}$ & $\begin{array}{l}4.3 \\
5.6 \\
5.2 \\
7.4 \\
8.0\end{array}$ & 6 & $\begin{array}{l}5.6 \\
4.9 \\
4.6 \\
4.3 \\
4.3\end{array}$ & $\begin{array}{l}2.8 \\
3.1 \\
2.9 \\
2.8 \\
2.8\end{array}$ & $\begin{array}{l}4.1 \\
3.8 \\
3.8 \\
3.8 \\
3.5\end{array}$ & $\begin{array}{l}7.0 \\
6.2 \\
5.6 \\
5.9 \\
7.1\end{array}$ & $\begin{array}{l}6.6 \\
6.6 \\
6.6 \\
6.6 \\
5.9\end{array}$ & $\begin{array}{l}7.6 \\
7.6 \\
7.3 \\
7.0 \\
6.6\end{array}$ & $\begin{array}{l}5.6 \\
5.3 \\
5.3 \\
5.3 \\
5.0\end{array}$ & $\begin{array}{l}6.6 \\
5.6 \\
5.2 \\
5.2 \\
4.6\end{array}$ & $\begin{array}{l}3.8 \\
3.6 \\
3.8 \\
3.6 \\
3.6\end{array}$ & \\
\hline $\begin{array}{l}26 \\
27 \\
28 \\
29 \\
20\end{array}$ & 8.0 & $\begin{array}{l}5.9 \\
6.4 \\
5.6 \\
4.9 \\
4.6\end{array}$ & 4.2 & $\begin{array}{l}2.8 \\
2.8 \\
2.8 \\
2.9 \\
3.0 \\
3.7\end{array}$ & $\begin{array}{l}3.4 \\
3.2 \\
3.2 \\
3.2 \\
3.1\end{array}$ & $\begin{array}{l}8.4 \\
8.0 \\
7.0 \\
6.6 \\
5.3 \\
4.7\end{array}$ & $\begin{array}{l}5.6 \\
6.2 \\
7.0 \\
6.2 \\
5.3 \\
4.7\end{array}$ & $\begin{array}{r}6.2 \\
6.6 \\
7.3 \\
\end{array}$ & $\begin{array}{l}5.0 \\
4.7 \\
4.7 \\
4.7 \\
4.4 \\
4.4\end{array}$ & $\begin{array}{l}4.3 \\
4.3 \\
4.0 \\
4.0 \\
3.8\end{array}$ & $\begin{array}{l}3.8 \\
3.8 \\
6.9 \\
5.2 \\
3.9 \\
3.6\end{array}$ & \\
\hline
\end{tabular}

\begin{tabular}{|c|c|c|c|c|c|c|}
\hline \multirow{2}{*}{ Month } & \multicolumn{3}{|c|}{ Million gallons a day } & \multirow{2}{*}{$\begin{array}{l}\text { Second- } \\
\text { feet } \\
\text { (mean) }\end{array}$} & \multicolumn{2}{|c|}{ Total run-off } \\
\hline & Maximum & Minimum & Mean & & $\begin{array}{l}\text { Million } \\
\text { gallons }\end{array}$ & Acre-feet \\
\hline $\begin{array}{l}\text { July } \\
\text { August } \\
\text { September. } \\
\text { October } \\
\text { November } \\
\text { December } \\
\text { January } \\
\text { February } \\
\text { March } \\
\text { April } \\
\text { May }\end{array}$ & $\begin{array}{l}8.0 \\
7.3 \\
7.6 \\
8.4 \\
7.6 \\
7.6 \\
8.0 \\
8.7 \\
6.9 \\
5.6\end{array}$ & \begin{tabular}{r|}
3.2 \\
2.8 \\
2.5 \\
3.0 \\
3.8 \\
3.4 \\
4.4 \\
3.8 \\
3.5 \\
2.5
\end{tabular} & $\begin{array}{l}\text { 5. } 72 \\
5.58 \\
5.54 \\
\text { 3. } 33 \\
4.73 \\
6.51 \\
5.66 \\
5.27 \\
6.37 \\
5.53 \\
4.09 \\
\text { 3. } 10\end{array}$ & $\begin{array}{r}8.85 \\
8.63 \\
8.57 \\
5.15 \\
7.32 \\
10.1 \\
8.76 \\
8.15 \\
9.86 \\
8.56 \\
6.33 \\
4.80\end{array}$ & $\begin{array}{l}177 \\
173 \\
166 \\
103 \\
142 \\
202 \\
175 \\
148 \\
198 \\
166 \\
137 \\
93.1\end{array}$ & $\begin{array}{l}\mathbf{5 4 4} \\
\mathbf{5 3 1} \\
510 \\
317 \\
\mathbf{4 3 5} \\
\mathbf{6 1 9} \\
\mathbf{5 3 8} \\
453 \\
606 \\
509 \\
389 \\
\mathbf{2 8 5}\end{array}$ \\
\hline The year..... & 8.7 & -..- & 5.12 & 7.92 & 1,870 & 5,740 \\
\hline
\end{tabular}

- Estimatod. 


\section{OHEO STREAM AT ELEVATION 1,560 FEET, NEAR KIPAEULU, MAUI}

Location.-Water-stage recorder at old diversion dam $2 \frac{1}{4}$ miles above mouth and 2 miles northwest of Kipahulu.

DrainagE AREA. -5.8 square miles.

Records available. - February, 1927, to June, 1929.

EXTREMEs.-Maximum discharge during period, 3,340 million gallons a day or 5,170 second-feet Dec. 16 (gage height, 15.45 feet); dry several days during the year.

1927-1929: Maximum discharge, that of Dec. 16, 1928; stream becomes dry during dry periods.

REMARKs.-Records good for ordinary stages; poor for high stages. No diversions.

Discharge, in million gallons a day, 1928-29

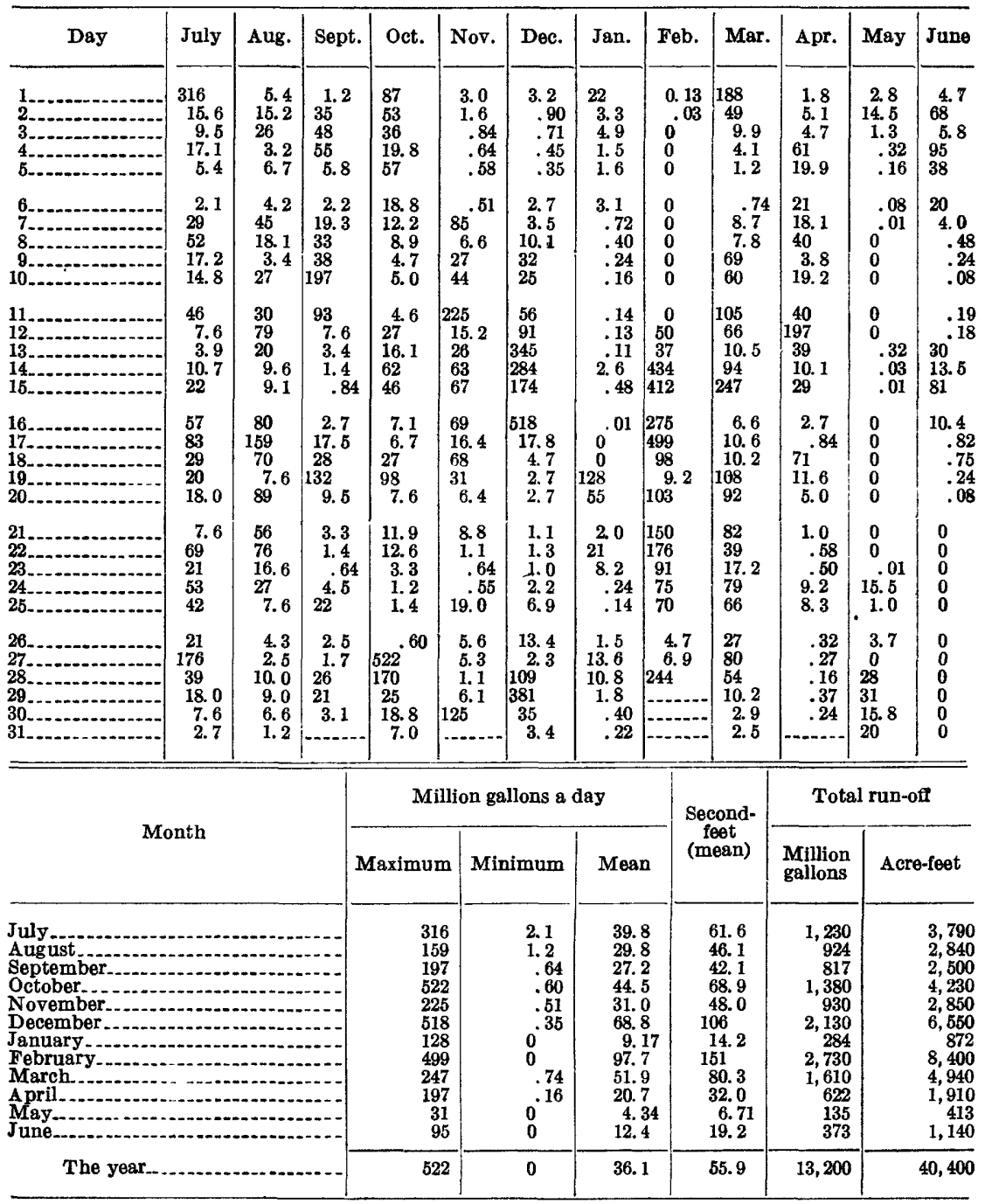




\section{RIGHT BRATCH OF KAHALAWE STREAM NEAR IIPAHULU, MAUI}

Location.- Water-stage recorder at old ditch intake, elevation 1,100 feet, 2 miles north of Kipahulu.

Drainage area. - 0.1 square mile.

Records available. - February, 1927, to June, 1929.

ExtrRmes.-Maximum discharge during year, 347 million gallons a day or 537 second-feet Feb. 17 (gage height, 10.07 feet); minimum, 0.3 million gallons a day or 0.5 second-foot June 28-30.

1927-1929: Maximum discharge, that of Feb. 17, 1929; minimum, 0.2 million gallons a day or 0.3 second-foot Mar. 23, 24, 1928.

REMARKs.- Records good for ordinary stages, poor for high stages. No diversion.

Discharge, in million gallons a day, 1928-29

\begin{tabular}{|c|c|c|c|c|c|c|c|c|c|c|c|c|}
\hline Day & July & Aug. & Sept. & Oct. & Nov. & Dec. & Jan. & Feb. & Mar. & Apr. & May & June \\
\hline $\begin{array}{l}1 . . \\
2 \\
3 .- \\
4 \\
5 \ldots\end{array}$ & $\begin{array}{l}9.6 \\
5.5 \\
3.3 \\
3.3 \\
2.3\end{array}$ & $\begin{array}{l}2.7 \\
3.2 \\
4.4 \\
2.3 \\
3.0\end{array}$ & $\begin{array}{l}2.0 \\
4.8 \\
4.2 \\
2.4 \\
2.0\end{array}$ & $\begin{array}{l}2.0 \\
2.8 \\
2.0 \\
2.6 \\
4.8\end{array}$ & $\begin{array}{l}1.5 \\
1.2 \\
1.1 \\
1.0 \\
1.0\end{array}$ & $\begin{array}{l}1.4 \\
1.2 \\
1.4 \\
1.1 \\
1.0\end{array}$ & $\begin{array}{l}3.5 \\
1.9 \\
2.1 \\
1.8 \\
1.6\end{array}$ & $\begin{array}{r}0.9 \\
.9 \\
.9 \\
.8 \\
.8\end{array}$ & $\begin{array}{l}5.4 \\
3.0 \\
2.1 \\
2.4 \\
2.0\end{array}$ & $\begin{array}{l}2.3 \\
2.7 \\
2.1 \\
7.8 \\
4.0\end{array}$ & $\begin{array}{r}1.6 \\
2.0 \\
1.8 \\
1.0 \\
.9\end{array}$ & $\begin{array}{l}1.1 \\
2.3 \\
2.8 \\
1.8\end{array}$ \\
\hline $\begin{array}{l}6 \\
7 \\
8 \\
9\end{array}$ & $\begin{array}{l}2.0 \\
3.3 \\
3.6 \\
2.1 \\
2.0\end{array}$ & $\begin{array}{l}3.0 \\
4.4 \\
2.7 \\
2.0 \\
4.0\end{array}$ & $\begin{array}{r}1.8 \\
7.2 \\
3.6 \\
5.3 \\
11.0\end{array}$ & $\begin{array}{l}2.1 \\
2.3 \\
1.9 \\
1.5 \\
1.6\end{array}$ & $\begin{array}{l}1.1 \\
7.1 \\
1.5 \\
1.9 \\
1.5\end{array}$ & $\begin{array}{l}1 . \\
1 . \\
1 . \\
2 . \\
1 .\end{array}$ & $\begin{array}{l}1 . \\
1 . \\
1 . \\
1 . \\
1 .\end{array}$ & $\begin{array}{r}.8 \\
1.8 \\
1.4 \\
.8 \\
1.0\end{array}$ & $\begin{array}{l}3 \\
4 \\
6\end{array}$ & $\begin{array}{l}3 . \\
3 . \\
4 . \\
1 . \\
6 .\end{array}$ & $\begin{array}{l}.9 \\
.8 \\
.8 \\
.7 \\
.7\end{array}$ & 1. \\
\hline 4 & $\begin{array}{l}5.6 \\
2.0 \\
1.6 \\
1.6 \\
1.5\end{array}$ & $\begin{array}{l}3.9 \\
3.8 \\
2.7 \\
2.3 \\
2.1\end{array}$ & $\begin{array}{l}4 . \\
2 . \\
2 . \\
2 . \\
1 .\end{array}$ & $\begin{array}{l}2 . \\
5 . \\
2 . \\
2 . \\
2 .\end{array}$ & $\begin{array}{l}3 . \\
1 . \\
1 . \\
7 . \\
2 .\end{array}$ & $\begin{array}{r}2 . \\
2 . \\
10 . \\
8 . \\
9 .\end{array}$ & $\begin{array}{l}1 . \\
1 . \\
1 . \\
1 . \\
1 .\end{array}$ & $\begin{array}{l}2.5 \\
.9 \\
1.0 \\
3.2 \\
7.1\end{array}$ & $\begin{array}{r}10.2 \\
5.6 \\
2.4 \\
12.6 \\
17.4\end{array}$ & $\begin{array}{r}5 . \\
12 . \\
3 . \\
2 . \\
2 .\end{array}$ & $\begin{array}{r}.7 \\
.9 \\
2.4 \\
1.6 \\
1.1\end{array}$ & $\begin{array}{l}1 . \\
1 . \\
6 .\end{array}$ \\
\hline , & $\begin{array}{l}3.5 \\
4.8 \\
3.5 \\
3.6 \\
4.3\end{array}$ & $\begin{array}{r}3.0 \\
10.6 \\
5.1 \\
2.1 \\
14.4\end{array}$ & $\begin{array}{l}2.3 \\
4.2 \\
4.0 \\
7.2 \\
2.1\end{array}$ & $\begin{array}{l}1 . \\
1 . \\
4 . \\
5 . \\
1 .\end{array}$ & $\begin{array}{l}6.7 \\
2.1 \\
2.1 \\
3.0 \\
1.9\end{array}$ & $\begin{array}{r}19.8 \\
6.0 \\
2.7 \\
2.4 \\
2.1\end{array}$ & $\begin{array}{r}1.0 \\
.9 \\
1.0 \\
6.9 \\
2.2\end{array}$ & $\begin{array}{c}15.7 \\
31 \\
8.0 \\
4.0 \\
6.5\end{array}$ & $\begin{array}{l}2 . \\
2 . \\
2 . \\
3 . \\
2 .\end{array}$ & $\begin{array}{l}4 . \\
1 . \\
1 .\end{array}$ & $\begin{array}{l}.7 \\
.6 \\
.5 \\
.5 \\
.5\end{array}$ & 1. \\
\hline 4 & $\begin{array}{l}2.3 \\
8.8 \\
2.4 \\
5.6 \\
4.6\end{array}$ & $\begin{array}{r}9.3 \\
11.7 \\
4.0 \\
6.3 \\
3.0\end{array}$ & $\begin{array}{l}1.9 \\
1.8 \\
1.5 \\
3.7 \\
7.4\end{array}$ & $\begin{array}{l}1.1 \\
1.5 \\
1.1 \\
1.0 \\
1.4\end{array}$ & $\begin{array}{l}1.9 \\
1.5 \\
1.4 \\
1.6 \\
2.1\end{array}$ & $\begin{array}{l}2.1 \\
2.0 \\
1.9 \\
2.1 \\
4.7\end{array}$ & $\begin{array}{r}1.1 \\
1.4 \\
1.1 \\
.9 \\
.8\end{array}$ & $\begin{array}{l}4.9 \\
8.8 \\
4.9 \\
8.9 \\
4.5\end{array}$ & $\begin{array}{l}2.4 \\
3.5 \\
3.1 \\
5.3 \\
4.2\end{array}$ & $\begin{array}{l}1 . \\
1 . \\
1 . \\
2 . \\
1 .\end{array}$ & $\begin{array}{r}.6 \\
.6 \\
2.2 \\
1.7 \\
.8\end{array}$ &. \\
\hline $\begin{array}{l}26 \\
27 \\
28 \\
30\end{array}$ & $\begin{array}{r}3.4 \\
10.6 \\
3.3 \\
3.0 \\
2.7 \\
2.1\end{array}$ & $\begin{array}{l}2.7 \\
2.7 \\
4.3 \\
3.6 \\
2.3 \\
1.9\end{array}$ & $\begin{array}{l}1.9 \\
1.8 \\
2.6 \\
3.5 \\
1.6 \\
\ldots .-\end{array}$ & $\begin{array}{l}1.0 \\
6.2 \\
9.9 \\
2.4 \\
3.4 \\
1.5\end{array}$ & $\begin{array}{l}1.5 \\
1.4 \\
1.5 \\
3.7 \\
5.8\end{array}$ & $\begin{array}{r}2.7 \\
1.6 \\
2.9 \\
15.9 \\
2.8 \\
1.8\end{array}$ & $\begin{array}{l}6.8 \\
7.7 \\
4.5 \\
1.5 \\
1.1 \\
1.0\end{array}$ & $\begin{array}{r}2.7 \\
2.4 \\
5.8 \\
\end{array}$ & $\begin{array}{r}4.5 \\
18.4 \\
12.6 \\
3.0 \\
2.3 \\
2.1\end{array}$ & $\begin{array}{l}1.4 \\
1.4 \\
1.1 \\
1.5 \\
1.2\end{array}$ & $\begin{array}{l}1.0 \\
1.1 \\
2.8 \\
3.8 \\
1.8 \\
3.7\end{array}$ & .4 \\
\hline
\end{tabular}

\begin{tabular}{|c|c|c|c|c|c|c|}
\hline \multirow{2}{*}{ Month } & \multicolumn{3}{|c|}{ Million gallons a day } & \multirow{2}{*}{$\begin{array}{l}\text { Second- } \\
\text { feet } \\
\text { (mean) }\end{array}$} & \multicolumn{2}{|c|}{ Total run-off } \\
\hline & Maximum & Minimum & - Mean & & $\begin{array}{l}\text { Million } \\
\text { gallons }\end{array}$ & Acre-feet \\
\hline $\begin{array}{l}\text { July } \\
\text { August } \\
\text { September } \\
\text { October } \\
\text { November } \\
\text { December } \\
\text { January } \\
\text { February } \\
\text { March } \\
\text { April } \\
\text { May }\end{array}$ & \begin{tabular}{r|r|}
10.6 \\
14.4 \\
11.0 \\
9.9 \\
7.5 \\
19.8 \\
7.7 \\
31 \\
18.4 \\
12.3 \\
3.8 \\
6.6
\end{tabular} & $\begin{array}{r}1.5 \\
1.9 \\
1.5 \\
1.0 \\
1.0 \\
1.0 \\
.8 \\
.8 \\
1.6 \\
1.1 \\
.5 \\
.3\end{array}$ & $\begin{array}{l}\text { 3. } 80 \\
\text { 4. 31 } \\
\text { 3. } 48 \\
2.65 \\
2.47 \\
\text { 3. } 96 \\
2.05 \\
\text { 4. } 75 \\
\text { 5. } 07 \\
\text { 3. } 01 \\
1.32 \\
1.22\end{array}$ & $\begin{array}{l}5.88 \\
6.67 \\
5.38 \\
4.10 \\
3.82 \\
6.13 \\
3.17 \\
7.35 \\
7.84 \\
4.66 \\
2.04 \\
1.89\end{array}$ & $\begin{array}{c}118 \\
134 \\
104 \\
82.3 \\
74.2 \\
123 \\
63.6 \\
133 \\
157 \\
90.4 \\
40.8 \\
36.5\end{array}$ & $\begin{array}{l}362 \\
410 \\
320 \\
252 \\
227 \\
377 \\
195 \\
408 \\
482 \\
277 \\
126 \\
112\end{array}$ \\
\hline The year.- & 31 & .3 & 3. 17 & 4.90 & 1,160 & 3,550 \\
\hline
\end{tabular}




\section{HATAWI STREAM NEAR NAHIKD, MAUI}

Locatron.-Water-stage recorder 200 feet above Koolau ditch intake and trail, $13 / 4$ mlles southeast of Nahiku, and $41 / 2$ miles southeast of Keanae.

Drain aGe AREA. - 0.8 square mile.

RECoRDS AV AILABLE.-January, 1914, to January, 1916; November, 1921, to June, 1929.

EXTREMEs.-Maximum discharge during year, 400 million gallons a day or 619 second-feet Dec. 16 (gage height, 6.00 feet); minimum, 2.2 million gallons a day or 3.4 second-feet June 29 .

1914-1916; 1921-1929: Maximum stage from floodmarks, about 20 feet Jan. 18, 1916 (discharge not determined); minimum, 1.4 million gallons a day or 2.2 second-feet July $5,8,1926$.

REMARKs.-Records good for ordinary stages; fair for estimated periods; poor for extremely high and low stages. No diversions.

Discharge, in million gallons a day, 1928-29

\begin{tabular}{|c|c|c|c|c|c|c|c|c|c|c|c|c|}
\hline Day & July & Aug. & Sept. & Oct. & Nov. & Dec. & Jan. & Feb. & Mar. & Apr. & May & June \\
\hline $\begin{array}{l}1 \\
2 \ldots- \\
3 \\
4 \\
5 \\
5\end{array}$ & $\begin{array}{l}65 \\
8.2 \\
7.2 \\
8.5 \\
4.5\end{array}$ & $\begin{array}{l}15.6 \\
10.3 \\
12.0 \\
11.2 \\
12.0\end{array}$ & $\begin{array}{r}-5.3 \\
23 \\
14.8 \\
14.0 \\
9.6\end{array}$ & $\begin{array}{r}11.1 \\
10.1 \\
5.6 \\
5.6 \\
7.8\end{array}$ & $\begin{array}{l}3.8 \\
3.5 \\
3.3 \\
3.5 \\
3.9\end{array}$ & $\begin{array}{r}5.8 \\
5.6 \\
5.3 \\
5.1 \\
50\end{array}$ & $\begin{array}{r}13.2 \\
8.2 \\
35 \\
10.3 \\
8.6\end{array}$ & $\begin{array}{l}4.9 \\
4.9 \\
5.0 \\
5.0 \\
4.8\end{array}$ & $\begin{array}{l}46 \\
27 \\
24 \\
49 \\
51\end{array}$ & $\begin{array}{l}4.2 \\
4.1 \\
4.2 \\
3.9 \\
3.5\end{array}$ & $\begin{array}{l}4.0 \\
3.9 \\
3.8 \\
3.6 \\
3.7\end{array}$ & $\begin{array}{l}2.6 \\
2.5 \\
2.5 \\
3.8 \\
5.6\end{array}$ \\
\hline $\begin{array}{r}6 \\
7-2 \\
8 \\
9 \\
90 \\
10\end{array}$ & $\begin{array}{r}4.0 \\
7.3 \\
11.3 \\
5.9 \\
6.1\end{array}$ & $\begin{array}{r}9.6 \\
8.6 \\
8.0 \\
7.3 \\
10.1\end{array}$ & $\begin{array}{r}7.7 \\
10.8 \\
7.1 \\
10.0 \\
32\end{array}$ & 5. 5 & $\begin{array}{r}3.5 \\
40 \\
7.0 \\
15.1 \\
31\end{array}$ & $\begin{array}{l}30 \\
9.6 \\
13 \\
14.6\end{array}$ & $\begin{array}{r}15.4 \\
8.4 \\
8.1 \\
7.1 \\
6.4\end{array}$ & $\begin{array}{l}6.3 \\
7.0 \\
5.2 \\
4.7 \\
4.4\end{array}$ & $\begin{array}{l}\mathbf{2 6} \\
\mathbf{5 7} \\
\mathbf{4 0} \\
\mathbf{7 9} \\
\mathbf{3 4}\end{array}$ & \begin{tabular}{r|}
3.5 \\
4.0 \\
8.0 \\
4.1 \\
43
\end{tabular} & $\begin{array}{l}4.8 \\
4.3 \\
4.5 \\
3.5 \\
3.7\end{array}$ & $\begin{array}{l}4.2 \\
3.2 \\
2.7 \\
2.6 \\
2.6\end{array}$ \\
\hline 11 & $\begin{array}{l}7.3 \\
5.1 \\
5.1 \\
5.5 \\
4.5\end{array}$ & $\begin{array}{l}8.4 \\
8.8 \\
8.2 \\
7.3 \\
7.1\end{array}$ & $\begin{array}{l}26 \\
8.6 \\
6.9 \\
6.0 \\
5.5\end{array}$ & $b_{22}$ & $\begin{array}{l}89 \\
8.5 \\
12.7 \\
31 \\
11.2\end{array}$ & $\begin{array}{l}14.7 \\
29 \\
51 \\
74 \\
28\end{array}$ & $\begin{array}{l}6.2 \\
5.8 \\
5.7 \\
9.8 \\
7.5\end{array}$ & \begin{tabular}{c|c}
4.2 \\
29 \\
6.3 \\
58 \\
99
\end{tabular} & $\begin{array}{l}37 \\
15.0 \\
12.0 \\
.11 .2 \\
33\end{array}$ & $\begin{array}{r}100 \\
145 \\
24 \\
9.7 \\
7.6\end{array}$ & \begin{tabular}{c|}
8.2 \\
7.0 \\
20 \\
10.3 \\
6.8
\end{tabular} & $\begin{array}{r}4.4 \\
4.0 \\
5.1 \\
3.4 \\
11.1\end{array}$ \\
\hline $\begin{array}{l}16-.- \\
17-. \\
18=- \\
19=- \\
20 \ldots\end{array}$ & $\begin{array}{r}18.1 \\
12.6 \\
8.3 \\
8.1 \\
6.1\end{array}$ & $\begin{array}{r}18.4 \\
29 \\
18.0 \\
8.4 \\
9.5\end{array}$ & $\begin{array}{r}5.6 \\
5.3 \\
5.0 \\
27 \\
6.6\end{array}$ & $\begin{array}{l}\text { 3. } 3 \\
\text { 3. } 3 \\
3.3 \\
3.2\end{array}$ & $\begin{array}{r}7.1 \\
6.3 \\
8.8 \\
7.6 \\
39\end{array}$ & $\begin{array}{r}102 \\
13.0 \\
15.6 \\
10.8 \\
9.6\end{array}$ & $\begin{array}{r}6.4 \\
5.6 \\
5.6 \\
10.9 \\
9.1\end{array}$ & $\begin{array}{r}112 \\
191 \\
97 \\
34 \\
109\end{array}$ & $\begin{array}{r}12.0 \\
10.3 \\
9.6 \\
8.8 \\
8.7\end{array}$ & \begin{tabular}{r|}
5.7 \\
5.3 \\
15.7 \\
6.1 \\
5.2
\end{tabular} & $\begin{array}{l}4.8 \\
4.0 \\
3.7 \\
3.5 \\
3.3\end{array}$ & $\begin{array}{l}\text { 6. } 1 \\
3.5 \\
3.1 \\
2.9 \\
2.7\end{array}$ \\
\hline $\begin{array}{l}21 \\
22 \\
24\end{array}$ & $\begin{array}{r}5.3 \\
12.0 \\
8.8 \\
28 \\
41\end{array}$ & $\begin{array}{r}9.2 \\
9.7 \\
12.3 \\
14.1 \\
7.7\end{array}$ & $\begin{array}{l}5.6 \\
5.2 \\
4.9 \\
4.7 \\
4.5\end{array}$ & $\begin{array}{l}\text { 3. } 4 \\
3.8 \\
3.5 \\
3.2 \\
3.1\end{array}$ & $\begin{array}{r}11.7 \\
8.1 \\
7.6 \\
7.6 \\
7.1\end{array}$ & $\begin{array}{l}10.3 \\
10.3 \\
10.3 \\
11.2 \\
47\end{array}$ & $\begin{array}{c}6.2 \\
163 \\
33 \\
8.8 \\
7.2\end{array}$ & $\begin{array}{l}86 \\
56 \\
14.0 \\
12.7 \\
10.3\end{array}$ & $\begin{array}{l}8.2 \\
7.5 \\
7.0 \\
6.7 \\
6.2\end{array}$ & $\begin{array}{l}5.0 \\
5.0 \\
5.0 \\
5.1 \\
4.8\end{array}$ & $\begin{array}{l}3.2 \\
3.1 \\
4.0 \\
5.3 \\
3.4\end{array}$ & $\begin{array}{l}2.7 \\
2.5 \\
2.5 \\
2.5 \\
2.5\end{array}$ \\
\hline $\begin{array}{l}26 \\
27 \\
28 \\
30\end{array}$ & $\begin{array}{r}117 \\
37 \\
11.2 \\
8.8 \\
8.6 \\
9.4\end{array}$ & $\begin{array}{r}6.5 \\
5.9 \\
10.0 \\
6.1 \\
65.7 \\
b 5.6\end{array}$ & $\begin{array}{l}4.3 \\
4.2 \\
4.1 \\
5.8 \\
4.4\end{array}$ & \begin{tabular}{c|}
3.0 \\
33 \\
21 \\
4.6 \\
4.3 \\
4.2
\end{tabular} & $\begin{array}{l}6.8 \\
6.6 \\
6.4 \\
6.6 \\
6.4\end{array}$ & $\begin{array}{l}27 \\
11.2 \\
15.9 \\
30 \\
11.2 \\
8.7\end{array}$ & $\begin{array}{l}6.4 \\
5.7 \\
5.3 \\
5.1 \\
5.0 \\
4.9\end{array}$ & 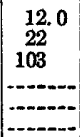 & $\begin{array}{l}5.8 \\
5.5 \\
5.1 \\
4.8 \\
4.5 \\
4.3\end{array}$ & $\begin{array}{l}4.7 \\
4.5 \\
4.3 \\
4.2 \\
4.1\end{array}$ & $\begin{array}{l}3.0 \\
5.7 \\
3.9 \\
3.0 \\
2.7 \\
2.7\end{array}$ & $\begin{array}{l}2.4 \\
2.3 \\
2.2 \\
2.2 \\
2.3\end{array}$ \\
\hline
\end{tabular}

\begin{tabular}{|c|c|c|c|c|c|c|}
\hline \multirow{2}{*}{ Month } & \multicolumn{3}{|c|}{ Million gallons a day } & \multirow{2}{*}{$\begin{array}{l}\text { Second- } \\
\text { feet } \\
\text { (mean) }\end{array}$} & \multicolumn{2}{|c|}{ Total run-off } \\
\hline & Maximum & Minimum & Mean & & $\begin{array}{l}\text { Million } \\
\text { gallons }\end{array}$ & Acre-feet \\
\hline $\begin{array}{l}\text { July } \\
\text { August } \\
\text { September } \\
\text { October } \\
\text { November } \\
\text { December } \\
\text { January } \\
\text { February } \\
\text { Mareh } \\
\text { April } \\
\text { May } \\
\text { June }\end{array}$ & $\begin{array}{r}117 \\
29 \\
32 \\
33 \\
89 \\
102 \\
163 \\
191 \\
79 \\
145 \\
20 \\
11.1\end{array}$ & $\begin{array}{l}4.0 \\
5.6 \\
4.1 \\
3.0 \\
3.3 \\
5.1 \\
4.9 \\
4.2 \\
4.3 \\
3.5 \\
2.7 \\
2.2\end{array}$ & $\begin{array}{r}15.9 \\
10.3 \\
9.48 \\
6.52 \\
13.7 \\
22.3 \\
14.3 \\
39.6 \\
21.2 \\
15.1 \\
4.88 \\
3.39\end{array}$ & $\begin{array}{l}24.6 \\
15.9 \\
14.7 \\
10.1 \\
21.2 \\
34.5 \\
22.1 \\
61.3 \\
32.8 \\
23.4 \\
7.55 \\
5.25\end{array}$ & $\begin{array}{r}493 \\
321 \\
284 \\
202 \\
411 \\
693 \\
444 \\
1,110 \\
656 \\
454 \\
151 \\
102\end{array}$ & $\begin{array}{r}1,510 \\
980 \\
873 \\
620 \\
1,260 \\
2,120 \\
1,360 \\
3,400 \\
2,020 \\
1,390 \\
464 \\
312\end{array}$ \\
\hline The year............... & 191 & $\overline{2.2}$ & 14.6 & 22.6 & 5,320 & 16,300 \\
\hline
\end{tabular}




\section{KAPAULA STREAM TEAR NAHIKU, MAUI}

Locatron.-Water-stage recorder 150 feet above intake to Koolau ditch, 300 feet above ditch trail, 13/4 miles southwest of Nahiku, and 4 miles southeast of Keanae.

Drainage area.- 0.2 square mile.

RmCORDS AVAILABLE.- November, 1921, to June, 1929.

EXTREMEs.- Maximum discharge during year, 665 million gallons a day or 1,030 second-feet Dec. 16 (gage height, 5.23 feet); minimum, 1.2 million gallons a day or 1.9 second-feet June 30 .

1022-1929: Maximum discharge, 868 million gallons a day or 1,340 second-feet Dec. 13, 1921 (gage height, 8.45 feet); minimum, 0.6 million gallons a day or 0.9 second-foot July 5,1926 .

REMARKs. - Records good for ordinary stages, fair for estimated periods, and poor for high stages. No diversions.

Discharge, in million gallons a day, 1928-29

\begin{tabular}{|c|c|c|c|c|c|c|c|c|c|c|c|c|}
\hline Day & July & Aug. & Sept. & Oet. & Nov. & Dec. & Jan. & Fel & o. Mar. & Apr. & May & June \\
\hline $\begin{array}{l}1 \\
3 \ldots \\
5 \ldots\end{array}$ & $\begin{array}{c}48 \\
6.3 \\
5.6 \\
3.8 \\
2.8\end{array}$ & $\begin{array}{r}12.8 \\
7.9 \\
8.3 \\
7.7 \\
7.4\end{array}$ & $\begin{array}{r}3.5 \\
21 \\
13.5 \\
11.8 \\
7.9\end{array}$ & $\begin{array}{l}8.4 \\
9.3 \\
3.4 \\
3.4 \\
6.6\end{array}$ & $\begin{array}{l}2.2 \\
2.0 \\
1.8 \\
2.0 \\
2.9\end{array}$ & $\begin{array}{r}2.4 \\
2.3 \\
2.2 \\
2.1 \\
64\end{array}$ & $\begin{array}{c}11.8 \\
6.5 \\
30 \\
10.0 \\
7.4\end{array}$ & & 34 & $\begin{array}{l}2.2 \\
2.2 \\
2.3 \\
2.1 \\
1.9\end{array}$ & $\begin{array}{l}1.8 \\
1.6 \\
1.6 \\
1.6 \\
1.6\end{array}$ & $\begin{array}{l}1.6 \\
1.6 \\
1.6 \\
4.9 \\
9.0\end{array}$ \\
\hline $\begin{array}{r}6 \\
7 \\
8 \\
9 \\
9 \\
10 \\
\ldots\end{array}$ & $\begin{array}{r}2.4 \\
10.1 \\
13.2 \\
5.4 \\
5.9\end{array}$ & $\begin{array}{l}5.1 \\
4.7 \\
4.3 \\
4.0 \\
8.8\end{array}$ & $\begin{array}{r}5.4 \\
10.4 \\
6.1 \\
8.6 \\
32\end{array}$ & $\begin{array}{l}3.5 \\
2.6 \\
2.3 \\
2.0 \\
1.9\end{array}$ & $\begin{array}{c}2.3 \\
39 \\
7.5 \\
13.8 \\
23\end{array}$ & $\begin{array}{c}29 \\
9.6 \\
13.9 \\
16.7 \\
17.4\end{array}$ & $\begin{array}{r}14.5 \\
8.2 \\
10.1 \\
b 7.9 \\
b 4.6\end{array}$ & 2 & $\begin{array}{l}31 \\
64 \\
24\end{array}$ & $\begin{array}{r}1.8 \\
2.1 \\
7.6 \\
2.7 \\
42\end{array}$ & $\begin{array}{l}2.7 \\
2.9 \\
3.2 \\
2.0 \\
2.0\end{array}$ & $\begin{array}{l}5.2 \\
2.7 \\
2.0 \\
1.7 \\
1.7\end{array}$ \\
\hline 14 & $\begin{array}{l}6.8 \\
3.8 \\
4.3 \\
7.0 \\
6.6\end{array}$ & $\begin{array}{l}7.2 \\
8.0 \\
7.1 \\
4.9 \\
6.2\end{array}$ & $\begin{array}{l}20 \\
5.9 \\
4.0 \\
3.4 \\
3.1\end{array}$ & $\begin{array}{r}1.8 \\
1.8 \\
1.7 \\
21 \\
3.2\end{array}$ & $\begin{array}{l}70 \\
11.6 \\
14.8 \\
27 \\
13.7\end{array}$ & $\begin{array}{l}16.7 \\
28 \\
35 \\
58 \\
27\end{array}$ & $\begin{array}{r}a 3.6 \\
3.2 \\
3.1 \\
13.3 \\
7.9\end{array}$ & $\mathbf{3 0}$ & $\begin{array}{l}28 \\
11.1 \\
8.5 \\
7.8 \\
20\end{array}$ & $\begin{array}{r}94 \\
125 \\
19.1 \\
7.4 \\
7.9\end{array}$ & $\begin{array}{r}8.6 \\
8.3 \\
24 \\
13.3 \\
7.2\end{array}$ & $\begin{array}{r}2.8 \\
2.6 \\
4.9 \\
2.7 \\
13.8\end{array}$ \\
\hline 18 & $\begin{array}{r}25 \\
13.2 \\
7.9 \\
7.5 \\
4.4\end{array}$ & $\begin{array}{r}17.0 \\
30 \\
10.8 \\
5.2 \\
10.2\end{array}$ & $\begin{array}{r}3.4 \\
3.0 \\
3.0 \\
28 \\
4.4\end{array}$ & $\begin{array}{l}2.0 \\
1.8 \\
1.8 \\
1.8 \\
1.7\end{array}$ & $\begin{array}{r}7.2 \\
5.6 \\
12.0 \\
8.5 \\
40\end{array}$ & $\begin{array}{l}96 \\
10.5 \\
13.2 \\
9.3 \\
8.1\end{array}$ & $\begin{array}{l}4.6 \\
3.5 \\
3.6 \\
9.1 \\
6.6\end{array}$ & 110 & $\begin{array}{l}8.3 \\
6.1 \\
5.2 \\
5.1 \\
4.7\end{array}$ & $\begin{array}{r}4.3 \\
4.0 \\
15.4 \\
4.1 \\
\mathbf{3 . 1}\end{array}$ & $\begin{array}{l}2.9 \\
2.2 \\
2.0 \\
1.8 \\
1.8\end{array}$ & $\begin{array}{l}5.2 \\
2.6 \\
1.9 \\
1.7 \\
1.5\end{array}$ \\
\hline $\begin{array}{l}21 \\
22 \\
24\end{array}$ & $\begin{array}{l}4.1 \\
11.3 \\
8.8 \\
29 \\
40\end{array}$ & $\begin{array}{r}5.8 \\
8.1 \\
12.6 \\
11.0 \\
5.6\end{array}$ & $\begin{array}{l}3.0 \\
2.0 \\
2.2 \\
2.1 \\
2.0\end{array}$ & $\begin{array}{l}1.8 \\
2.2 \\
1.9 \\
1.7 \\
1.6\end{array}$ & $\begin{array}{r}11.9 \\
5.8 \\
4.3 \\
4.0 \\
3.4\end{array}$ & $\begin{array}{r}7.2 \\
6.3 \\
5.2 \\
7.4 \\
40\end{array}$ & $\begin{array}{c}4.7 \\
164 \\
21 \\
7.0 \\
5.1\end{array}$ & 34 & $\begin{array}{l}4.4 . \\
4.1 \\
3.9 \\
3.6 \\
3.4\end{array}$ & $\begin{array}{l}2.6 \\
2.2 \\
2.1 \\
2.3 \\
2.0\end{array}$ & $\begin{array}{l}1.8 \\
1.8 \\
3.1 \\
4.3 \\
2.1\end{array}$ & $\begin{array}{l}1.5 \\
1.4 \\
1.4 \\
1.4 \\
1.5\end{array}$ \\
\hline $\begin{array}{l}26 \\
27 \\
28 \\
30 \\
31\end{array}$ & \begin{tabular}{|c|}
114 \\
25 \\
7.9 \\
6.8 \\
6.6 \\
7.0
\end{tabular} & $\begin{array}{l}4.1 \\
6.2 \\
6.4 \\
3.6 \\
2.9 \\
2.7\end{array}$ & $\begin{array}{l}1.8 \\
1.9 \\
1 . \\
\mathbf{2} .\end{array}$ & $\begin{array}{r}1.6 \\
32 \\
11.8 \\
2.8 \\
3.5 \\
2.8\end{array}$ & $\begin{array}{l}3.0 \\
2.8 \\
2.7 \\
2.8 \\
2.9\end{array}$ & $\begin{array}{c}23 \\
8.7 \\
13.2 \\
25 \\
9.4 \\
6.1\end{array}$ & $\begin{array}{l}4.6 \\
4.1 \\
4.0 \\
3.6 \\
3.2 \\
2.9\end{array}$ & : & $\begin{array}{l}3.0 \\
2.8 \\
2.6 \\
2.4 \\
2.2 \\
2.2\end{array}$ & $\begin{array}{r}1.9 \\
1.8 \\
1.8 \\
1.7 \\
1.7 \\
\\
-.-\end{array}$ & $\begin{array}{l}1.8 \\
4.9 \\
2.9 \\
1.9 \\
1.8 \\
1.7\end{array}$ & $\begin{array}{l}1.4 \\
1.4 \\
1.4 \\
1.8 \\
1.8 \\
\cdots\end{array}$ \\
\hline \multirow{2}{*}{\multicolumn{4}{|c|}{ Month }} & \multicolumn{5}{|c|}{ Million gallons a day } & & \multicolumn{3}{|c|}{ Total run-off } \\
\hline & & & & Maximum & $\mathbf{M i}$ & limum & Mear & & & $\begin{array}{l}\text { Million } \\
\text { gaillons }\end{array}$ & Acr & e-feet \\
\hline $\begin{array}{l}\text { July-. } \\
\text { August. } \\
\text { September. } \\
\text { October } \\
\text { November. } \\
\text { December } \\
\text { January } \\
\text { February... } \\
\text { March } \\
\text { April. } \\
\text { May. } \\
\text { Juno }\end{array}$ & & & & \begin{tabular}{r}
114 \\
30 \\
32 \\
32 \\
70 \\
96 \\
164 \\
\hdashline 125 \\
24 \\
13.3
\end{tabular} & & $\begin{array}{l}2.4 \\
2.7 \\
1.9 \\
1.6 \\
1.8 \\
2.1 \\
2.9 \\
2.2 \\
1.7 \\
1.6 \\
1.3\end{array}$ & \multicolumn{2}{|c|}{$\begin{array}{c}14.5 \\
7.95 \\
7.45 \\
4.70 \\
11.7 \\
19.8 \\
12.7 \\
38.6 \\
16.0 \\
12.4 \\
3.91 \\
2.82\end{array}$} & $\begin{array}{c}22.4 \\
12.3 \\
11.5 \\
7.27 \\
18.1 \\
30.6 \\
19.6 \\
59.7 \\
24.8 \\
19.2 \\
6.05 \\
4.36\end{array}$ & $\begin{array}{c}450 \\
247 \\
224 \\
146 \\
350 \\
613 \\
394 \\
1,080 \\
496 \\
371 \\
121 \\
84.7\end{array}$ & \multicolumn{2}{|r|}{$\begin{array}{r}1,380 \\
756 \\
686 \\
447 \\
1,080 \\
1,880 \\
1,210 \\
3,320 \\
1,520 \\
1,140 \\
372 \\
260\end{array}$} \\
\hline \multicolumn{3}{|l|}{ The year. } & & $\cdots$ & & 1.3 & 12. & & 19.3 & 4,580 & & 14,100 \\
\hline
\end{tabular}

- Partly estimated.

bstimated. 


\section{KOOLAU DITCH AT NAHIKU WEIR, NEAR NAHIKU, MAUI}

Location.-Water-stage recorder between Kapaula and Waiohue Streams, 31/2 miles southwest of Nahiku and 4 miles southeast of Keanae.

Records avaILABLE.-February, 1919, to June, 1929.

EXTREMES.-Maximum discharge during year, 56.8 million gallons a day or 87.9 second-feet some time between July 12 and Sept. 1; no flow Feb. 16-21 (water shut out of ditch).

1919-1929: Maximum discharge, that between July 12 and Sept. 1, 1928; no flow occasionally when intake gates are closed.

REMARKs.-Records excellent except those for extremely low stages, which are good; estimated records fair July 1-9, July 12 to Sept. 1, Sept. 3 to Oct. 15, Oct. 27 to Dec. 21 , and Dec. 31 to Jan. 23 by comparison with discharges from twice daily readings of first ditch staff gage above station. Regulated by spillways and gates. Koolau ditch diverts at elevation of 1,200 feet from all streams from Makapipi to Alo.

Discharge, in million gallons a day, 1928-29

\begin{tabular}{|c|c|c|c|c|c|c|c|c|c|c|c|c|}
\hline Day & July & Aug. & Sept. & Oct. & Nov. & Dec. & Jan. & Feb. & Mar. & Apr. & May & June \\
\hline $\begin{array}{l}1 \\
2 \\
3 \\
4 \\
5\end{array}$ & $\begin{array}{l}37 \\
35 \\
32 \\
29 \\
21\end{array}$ & $\begin{array}{l}42 \\
37 \\
38 \\
36 \\
38\end{array}$ & $\begin{array}{l}21 \\
35 \\
35 \\
39 \\
35\end{array}$ & $\begin{array}{l}14 \\
34 \\
21 \\
20 \\
27\end{array}$ & $\begin{array}{l}16 \\
14 \\
14 \\
13 \\
16\end{array}$ & $\begin{array}{l}18 \\
18 \\
17 \\
17 \\
32\end{array}$ & $\begin{array}{l}40 \\
32 \\
40 \\
39 \\
30\end{array}$ & $\begin{array}{l}16.7 \\
16.0 \\
15.3 \\
15.0 \\
14.2\end{array}$ & $\begin{array}{l}42 \\
44 \\
44 \\
44 \\
46\end{array}$ & $\begin{array}{l}15.0 \\
14.6 \\
14.6 \\
13.6 \\
12.3\end{array}$ & $\begin{array}{l}13.6 \\
12.3 \\
11.6 \\
11.0 \\
11.3\end{array}$ & $\begin{array}{r}8.4 \\
8.4 \\
8.1 \\
13.9 \\
18.8\end{array}$ \\
\hline $\begin{array}{r}6 . \\
7 . \\
8 \\
9 . \\
10 .\end{array}$ & $\begin{array}{l}20 \\
24 \\
35 \\
24 \\
28\end{array}$ & $\begin{array}{l}32 \\
31 \\
28 \\
25 \\
33\end{array}$ & $\begin{array}{l}28 \\
36 \\
30 \\
38 \\
40\end{array}$ & $\begin{array}{l}21 \\
19 \\
16 \\
16 \\
15\end{array}$ & $\begin{array}{l}14 \\
39 \\
25 \\
18 \\
21\end{array}$ & $\begin{array}{l}44 \\
35 \\
27 \\
42 \\
36\end{array}$ & $\begin{array}{r}40 \\
32 \\
17 \\
4.0 \\
4.0\end{array}$ & $\begin{array}{l}21 \\
23 \\
17.4 \\
15.0 \\
13.9\end{array}$ & $\begin{array}{l}44 \\
46 \\
46 \\
43 \\
36\end{array}$ & $\begin{array}{l}12.0 \\
13.4 \\
25 \\
15.0 \\
36\end{array}$ & $\begin{array}{l}15.0 \\
15.6 \\
16.2 \\
12.3 \\
12.6\end{array}$ & $\begin{array}{r}15.0 \\
10.7 \\
9.0 \\
8.4 \\
8.4\end{array}$ \\
\hline$\ldots$ & $\begin{array}{l}30 \\
24 \\
23 \\
23 \\
20\end{array}$ & $\begin{array}{l}30 \\
37 \\
33 \\
27 \\
28\end{array}$ & $\begin{array}{r}44 \\
39 \\
32 \\
26 \\
23\end{array}$ & $\begin{array}{l}15 \\
14 \\
13 \\
14 \\
16\end{array}$ & $\begin{array}{l}39 \\
\mathbf{3 0} \\
\mathbf{3 1} \\
\mathbf{3 4} \\
\mathbf{3 7}\end{array}$ & $\begin{array}{l}40 \\
43 \\
43 \\
44 \\
45\end{array}$ & $\begin{array}{l}11 \\
16 \\
16 \\
22 \\
23\end{array}$ & $\begin{array}{l}13.2 \\
27 \\
21 \\
33 \\
46\end{array}$ & $\begin{array}{l}42 \\
44 \\
42 \\
44 \\
49\end{array}$ & $\begin{array}{l}51 \\
51 \\
46 \\
42 \\
36\end{array}$ & $\begin{array}{l}21 \\
21 \\
36 \\
34 \\
26\end{array}$ & $\begin{array}{l}10.4 \\
10.8 \\
16.2 \\
11.3 \\
30 .\end{array}$ \\
\hline 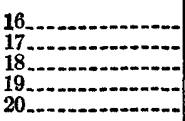 & $\begin{array}{l}31 \\
35 \\
32 \\
32 \\
27\end{array}$ & $\begin{array}{l}40 \\
44 \\
44 \\
35 \\
40\end{array}$ & $\begin{array}{l}23 \\
22 \\
19 \\
39 \\
26\end{array}$ & $\begin{array}{r}\text { a } 13.2 \\
13.2 \\
12.9 \\
12.9 \\
12.3\end{array}$ & $\begin{array}{l}33 \\
28 \\
34 \\
30 \\
34\end{array}$ & $\begin{array}{l}40 \\
37 \\
42 \\
37 \\
32\end{array}$ & $\begin{array}{l}19 \\
18 \\
19 \\
29 \\
28\end{array}$ & $\begin{array}{r}18.5 \\
0 \\
0 \\
0 \\
0\end{array}$ & $\begin{array}{l}\mathbf{4 2} \\
\mathbf{3 8} \\
\mathbf{3 6} \\
\mathbf{3 4} \\
\mathbf{3 2}\end{array}$ & $\begin{array}{l}28 \\
24 \\
43 \\
28 \\
23\end{array}$ & $\begin{array}{l}17.8 \\
15.6 \\
14.2 \\
13.2 \\
12.3\end{array}$ & $\begin{array}{r}18.1 \\
12.3 \\
10.7 \\
9.8 \\
9.3\end{array}$ \\
\hline - & $\begin{array}{l}23 \\
30 \\
33 \\
36 \\
38\end{array}$ & $\begin{array}{l}36 \\
33 \\
37 \\
42 \\
30\end{array}$ & $\begin{array}{l}22 \\
20 \\
18 \\
17 \\
16\end{array}$ & $\begin{array}{l}12.3 \\
13.9 \\
12.9 \\
12.3 \\
11.3\end{array}$ & $\begin{array}{l}36 \\
29 \\
25 \\
26 \\
23\end{array}$ & $\begin{array}{l}33 \\
34 \\
32 \\
36 \\
40\end{array}$ & $\begin{array}{l}20 \\
45 \\
44 \\
40 \\
32\end{array}$ & $\begin{array}{l}16.4 \\
28 \\
21 \\
34 \\
38\end{array}$ & $\begin{array}{l}30 \\
28 \\
26 \\
24 \\
23\end{array}$ & $\begin{array}{l}21 \\
18.9 \\
17.8 \\
17.8 \\
18.1\end{array}$ & $\begin{array}{l}12.0 \\
11.6 \\
14.6 \\
17.0 \\
12.0\end{array}$ & 9. \\
\hline 6. & $\begin{array}{l}32 \\
37 \\
35 \\
33 \\
33 \\
32\end{array}$ & $\begin{array}{l}32 \\
27 \\
35 \\
25 \\
23 \\
20\end{array}$ & $\begin{array}{r}15 \\
15 \\
15 \\
20 \\
16\end{array}$ & $\begin{array}{l}11.0 \\
27 \\
35 \\
19 \\
15 \\
14\end{array}$ & $\begin{array}{l}21 \\
20 \\
19 \\
18 \\
20\end{array}$ & $\begin{array}{l}42 \\
40 \\
40 \\
42 \\
40\end{array}$ & $\begin{array}{l}28 \\
24 \\
23 \\
20 \\
18.5 \\
17.4\end{array}$ & $\begin{array}{l}38 \\
40 \\
46\end{array}$ & $\begin{array}{l}21 \\
19.6 \\
18.5 \\
17.1 \\
16.0 \\
16.3\end{array}$ & $\begin{array}{l}15.6 \\
16.0 \\
14.2 \\
13.9 \\
13.6\end{array}$ & $\begin{array}{l}10.4 \\
16.7 \\
12.6 \\
10.1 \\
9.3 \\
9.0\end{array}$ & $\begin{array}{l}7.9 \\
7.3 \\
7.1 \\
6.8\end{array}$ \\
\hline
\end{tabular}

\begin{tabular}{|c|c|c|c|c|c|c|}
\hline \multirow{2}{*}{ Month } & \multicolumn{3}{|c|}{ Million gallons a day } & \multirow{2}{*}{$\begin{array}{c}\text { Second- } \\
\text { feet } \\
\text { (mean) }\end{array}$} & \multicolumn{2}{|c|}{ Total run-off } \\
\hline & Maximum & Minimum & Mean & & $\begin{array}{l}\text { Million } \\
\text { gallons }\end{array}$ & Acre-feet \\
\hline $\begin{array}{l}\text { July } \\
\text { August } \\
\text { September. } \\
\text { October } \\
\text { November. } \\
\text { December } \\
\text { January } \\
\text { February }(24 \text { days) } \\
\text { March. } \\
\text { April. } \\
\text { May. } \\
\text { June }\end{array}$ & $\begin{array}{l}38 \\
44 \\
\mathbf{4 4} \\
35 \\
39 \\
\mathbf{4 5} \\
\mathbf{4 5} \\
46 \\
\mathbf{4 9} \\
\mathbf{5 1} \\
36 \\
30\end{array}$ & $\begin{array}{l}20 \\
20 \\
15 \\
11.0 \\
13 \\
17 \\
4.0 \\
0 \\
16.3 \\
12.0 \\
9.0 \\
6.8\end{array}$ & $\begin{array}{l}29.8 \\
33.5 \\
26.8 \\
16.9 \\
25.2 \\
35.6 \\
25.5 \\
24.4 \\
34.7 \\
23.6 \\
15.4 \\
10.9\end{array}$ & $\begin{array}{l}46.1 \\
\mathbf{5 1 . 8} \\
41.5 \\
26.1 \\
39.0 \\
55.1 \\
39.5 \\
37.8 \\
53.7 \\
36.5 \\
23.8 \\
16.9\end{array}$ & $\begin{array}{r}924 \\
1,040 \\
803 \\
523 \\
757 \\
1,100 \\
791 \\
587 \\
1,080 \\
709 \\
478 \\
327\end{array}$ & $\begin{array}{l}2,840 \\
3,100 \\
2,470 \\
1,610 \\
2,320 \\
3,390 \\
2,430 \\
1,800 \\
3,300 \\
2,170 \\
1,470 \\
1,000\end{array}$ \\
\hline The year (361 days) & 51 & 0 & 25.3 & 39.1 & 9,120 & 28,000 \\
\hline
\end{tabular}

- Partly estimated. 
WAIOHUE STREAM NEAR NAHIKO, MAUI

Location.-Water-stage recorder 200 feet above intake to Koolau ditch, 300 feet above ditch trail, $21 / 4$ miles southwest of Nahiku, and $31 / 2$ miles southeast of Keanae.

Drainage AREa.- -1.5 square miles.

RECORDS AVAILABLE.-OCtober, 1921, to June, 1929.

ExTREMes.-Maximum discharge during year, 430 million gallons a day or 665 second-feet Feb. 17 (gage height, 4.87 feet); minimum, 3.0 million gallons a day or 4.6 second-feet Oct. 25,26 , and 27.

1922-1929: Maximum discharge, 544 million gallons a day or 842 secondfeet Apr. 17, 1927 (gage height, 5.63 feet); minimum, 1.7 million gallons a day or 2.6 second-feet Apr. 11, 1926.

Remarks.-Records good for ordinary stages; poor for extremely high and low stages. No diversions.

Discharge, in million gallons a day, 1928-29

\begin{tabular}{|c|c|c|c|c|c|c|c|c|c|c|c|c|}
\hline Day & July & Aug. & Sept. & Oct. & Nov. & Dec. & Jan. & Feb. & Mar. & Apr. & May & June \\
\hline $\begin{array}{l}1 \ldots \\
2 \ldots \\
3 \ldots \\
4 \ldots \\
5\end{array}$ & $\begin{array}{c}28 \\
7.2 \\
6.4 \\
5.8 \\
5.3\end{array}$ & $\begin{array}{r}13.3 \\
8.7 \\
9.9 \\
8.0 \\
8.8\end{array}$ & $\begin{array}{r}4.7 \\
18.4 \\
10.3 \\
8.7 \\
6.8\end{array}$ & $\begin{array}{r}13.1 \\
8.9 \\
4.7 \\
5.3 \\
6.6\end{array}$ & $\begin{array}{l}3.4 \\
3.4 \\
3.2 \\
3.6 \\
4.2\end{array}$ & $\begin{array}{r}4.2 \\
4.1 \\
4.0 \\
3.9 \\
30\end{array}$ & $\begin{array}{r}10.6 \\
6.6 \\
15.7 \\
7.6 \\
7.0\end{array}$ & $\begin{array}{l}4.7 \\
4.5 \\
4.2 \\
4.1 \\
4.0\end{array}$ & $\begin{array}{l}22 \\
14.4 \\
15.1 \\
24 \\
36\end{array}$ & $\begin{array}{l}3.8 \\
3.9 \\
4.0 \\
3.7 \\
3.5\end{array}$ & $\begin{array}{l}3.8 \\
3.5 \\
3.3 \\
3.2 \\
3.3\end{array}$ & $\begin{array}{l}3.1 \\
3.2 \\
3.1 \\
3.9 \\
4.7\end{array}$ \\
\hline $\begin{array}{r}6 \\
7 \\
8 \\
9 \\
10\end{array}$ & $\begin{array}{l}5.0 \\
8.4 \\
9.3 \\
6.6 \\
8.0\end{array}$ & $\begin{array}{l}6.4 \\
6.2 \\
5.9 \\
5.6 \\
7.9\end{array}$ & $\begin{array}{r}6.4 \\
10.0 \\
6.4 \\
10.5 \\
29\end{array}$ & $\begin{array}{l}4.8 \\
4.4 \\
4.4 \\
4.0 \\
3.8\end{array}$ & $\begin{array}{c}3.8 \\
23 \\
5.4 \\
7.2 \\
9.8\end{array}$ & $\begin{array}{r}17.1 \\
5.5 \\
9.0 \\
9.4 \\
10.7\end{array}$ & $\begin{array}{r}12.3 \\
7.0 \\
8.1 \\
6.2 \\
5.9\end{array}$ & $\begin{array}{l}6.6 \\
6.7 \\
4.6 \\
4.1 \\
3.9\end{array}$ & $\begin{array}{l}14.9 \\
32 \\
24 \\
54 \\
23\end{array}$ & $\begin{array}{r}3.5 \\
4.0 \\
6.0 \\
4.0 \\
37\end{array}$ & $\begin{array}{l}4.5 \\
4.5 \\
4.7 \\
3.5 \\
3.6\end{array}$ & $\begin{array}{l}3 . \\
3 . \\
3 .\end{array}$ \\
\hline$\ldots$ & $\begin{array}{l}8.0 \\
5.9 \\
5.6 \\
5.6 \\
5.4\end{array}$ & $\begin{array}{l}6.2 \\
8.3 \\
7.5 \\
5.9 \\
6.1\end{array}$ & $\begin{array}{r}13.4 \\
7.0 \\
6.2 \\
5.9 \\
5.6\end{array}$ & $\begin{array}{l}3.7 \\
3.6 \\
3.5 \\
4.4 \\
4.3\end{array}$ & $\begin{array}{r}32 \\
6.9 \\
11.2 \\
21 \\
11.7\end{array}$ & $\begin{array}{l}11.8 \\
16.5 \\
24 \\
33 \\
17.3\end{array}$ & $\begin{array}{l}5.6 \\
5.3 \\
5.1 \\
9.7 \\
6.2\end{array}$ & $\begin{array}{r}3.8 \\
12.3 \\
4.7 \\
23 \\
33\end{array}$ & $\begin{array}{r}28 \\
12.4 \\
9.8 \\
11.5 \\
15.7\end{array}$ & $\begin{array}{c}44 \\
54 \\
9.7 \\
5.9 \\
6.2\end{array}$ & $\begin{array}{r}4.4 \\
4.5 \\
10.8 \\
8.8 \\
5.4\end{array}$ & $\begin{array}{l}4 . \\
4 . \\
4 .\end{array}$ \\
\hline 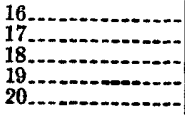 & $\begin{array}{r}17.4 \\
11.0 \\
8.7 \\
10.1 \\
7.0\end{array}$ & $\begin{array}{r}13.7 \\
24 \\
11.3 \\
6.2 \\
12.3\end{array}$ & $\begin{array}{r}5.6 \\
5.1 \\
5.5 \\
15.3 \\
5.3\end{array}$ & $\begin{array}{l}3.8 \\
3.6 \\
3.5 \\
3.6 \\
3.3\end{array}$ & $\begin{array}{r}7.8 \\
6.8 \\
10.2 \\
8.0 \\
21\end{array}$ & \begin{tabular}{r}
\multicolumn{1}{c}{59} \\
12.4 \\
14.3 \\
10.8 \\
8.7
\end{tabular} & $\begin{array}{l}5.4 \\
4.8 \\
5.1 \\
6.4 \\
5.6\end{array}$ & $\begin{array}{r}53 \\
100 \\
50 \\
22 \\
46\end{array}$ & $\begin{array}{l}8.0 \\
7.0 \\
6.6 \\
6.2 \\
5.9\end{array}$ & $\begin{array}{r}6.1 \\
6.2 \\
17.4 \\
6.2 \\
5.6\end{array}$ & $\begin{array}{l}4.2 \\
4.1 \\
4.1 \\
4.0 \\
3.9\end{array}$ & $\begin{array}{l}4 . \\
4 . \\
3, \\
3 .\end{array}$ \\
\hline 2425 & $\begin{array}{r}6.4 \\
11.2 \\
8.9 \\
21 \\
26\end{array}$ & $\begin{array}{l}7.0 \\
8.9 \\
9.5 \\
9.5 \\
6.2\end{array}$ & $\begin{array}{l}4.8 \\
4.6 \\
4.5 \\
4.4 \\
4.1\end{array}$ & $\begin{array}{l}3.3 \\
3.9 \\
3.3 \\
3.2 \\
3.2\end{array}$ & $\begin{array}{l}9.9 \\
6.6 \\
6.4 \\
6.2 \\
5.8\end{array}$ & $\begin{array}{r}8.0 \\
7.4 \\
6.8 \\
8.9 \\
34\end{array}$ & $\begin{array}{r}5.5 \\
104 \\
15.4 \\
7.6 \\
6.8\end{array}$ & $\begin{array}{l}31 \\
32 \\
12.4 \\
.14 .1 \\
8.4\end{array}$ & $\begin{array}{l}5.8 \\
5.3 \\
5.0 \\
4.8 \\
4.7\end{array}$ & $\begin{array}{l}5.1 \\
4.8 \\
4.8 \\
4.8 \\
4.4\end{array}$ & $\begin{array}{l}3.8 \\
3.7 \\
4.4 \\
4.2 \\
3.6\end{array}$ & $\begin{array}{l}3.8 \\
3 . \\
3.8 \\
3.2 \\
3 .\end{array}$ \\
\hline $\begin{array}{l}26 \\
27 \\
28 \\
28 \\
30 \\
31\end{array}$ & $\begin{array}{r}62 \\
22 \\
9.8 \\
8.9 \\
8.0 \\
8.3\end{array}$ & $\begin{array}{l}5.8 \\
6.2 \\
9.9 \\
5.4 \\
5.0 \\
4.8\end{array}$ & $\begin{array}{l}4.0 \\
4.0 \\
4.0 \\
4.7 \\
4.0\end{array}$ & $\begin{array}{r}3.0 \\
16.1 \\
7.2 \\
3.7 \\
3.3 \\
3.3\end{array}$ & $\begin{array}{l}5.4 \\
5.0 \\
4.8 \\
4.7 \\
4.6\end{array}$ & $\begin{array}{r}13.0 \\
7.0 \\
13.1 \\
18.9 \\
7.2 \\
6.6\end{array}$ & $\begin{array}{l}6.4 \\
6.1 \\
5.9 \\
5.6 \\
5.1 \\
4.8\end{array}$ & $\begin{array}{r}8.8 \\
14.1 \\
38\end{array}$ & $\begin{array}{l}4.5 \\
4.2 \\
4.1 \\
3.9 \\
3.8 \\
3.8\end{array}$ & $\begin{array}{l}4.2 \\
4.0 \\
3.9 \\
3.7 \\
3.8\end{array}$ & $\begin{array}{l}3.5 \\
3.8 \\
3.7 \\
3.4 \\
3.3 \\
3.2\end{array}$ & $\begin{array}{l}3.5 \\
3.3 \\
3.2 \\
3.2 \\
3.2\end{array}$ \\
\hline
\end{tabular}

\begin{tabular}{|c|c|c|c|c|c|c|}
\hline \multirow{2}{*}{ Month } & \multicolumn{3}{|c|}{ Million gallons a day } & \multirow{2}{*}{$\begin{array}{c}\text { Second- } \\
\text { feet } \\
\text { (mean) }\end{array}$} & \multicolumn{2}{|c|}{ Total run-off } \\
\hline & Maximum & Minimum & Mean & & $\begin{array}{l}\text { Million } \\
\text { gallons }\end{array}$ & Acre-feet \\
\hline $\begin{array}{l}\text { July } \\
\text { August. } \\
\text { September } \\
\text { October. } \\
\text { November. } \\
\text { December } \\
\text { January } \\
\text { February } \\
\text { March } \\
\text { April } \\
\text { May } \\
\text { Juno }\end{array}$ & $\begin{array}{l}62 \\
24 \\
29 \\
16.1 \\
32 \\
59 \\
104 \\
100 \\
54 \\
54 \\
10.8 \\
\mathbf{9 . 3}\end{array}$ & $\begin{array}{l}5.0 \\
4.8 \\
4.0 \\
3.0 \\
3.2 \\
3.9 \\
4.8 \\
3.8 \\
3.8 \\
3.5 \\
3.2 \\
3.1\end{array}$ & $\begin{array}{l}11.8 \\
8.40 \\
7.64 \\
4.86 \\
8.77 \\
14.1 \\
10.3 \\
19.8 \\
13.6 \\
9.27 \\
4.28 \\
3.90\end{array}$ & $\begin{array}{l}18.3 \\
13.0 \\
11.8 \\
7.52 \\
13.6 \\
21.8 \\
15.9 \\
30.6 \\
21.0 \\
14.3 \\
6.62 \\
6.03\end{array}$ & $\begin{array}{l}367 \\
260 \\
229 \\
151 \\
263 \\
437 \\
319 \\
554 \\
420 \\
278 \\
133 \\
117\end{array}$ & $\begin{array}{r}1,120 \\
799 \\
703 \\
462 \\
807 \\
1,340 \\
\mathbf{9 8 0} \\
1,700 \\
1,290 \\
853 \\
407 \\
369\end{array}$ \\
\hline The year..... & 104 & 3.0 & 9.67 & 15.0 & 3,530 & 10,800 \\
\hline
\end{tabular}


WEST KOPIIULA STREAM NEAR KEATAE, MAUI

Location.-Water-stage recorder 600 feet above Koolau ditch crossing and highway bridge and 3.miles southeast of Keanae post office.

Drainage area.-3.9 square miles.

ReCORDS AVAILABle.-January, 1914, to September, 1917; October, 1921, to June, 1929.

Extremes.-Maximum discharge during year, 807 million gallons a day or 1,250 second-feet Feb. 17 (gage height, 5.63 feet); minimum, 2.8 million gallons a day or 4.3 second-feet June 29.

1914-1917, 1921-1929: Maximum discharge, about 2,000 million gallons a day or 3,090 second-feet Jan. 18, 1916 (gage height, 9.25 feet); minimum, 0.6 million gallons a day or 0.9 second-feet Sept. 15-17, 1917 .

REMARKS.-Records good for ordinary stages, fair for estimated periods, and poor for high stages. No diversions.

Discharge, in million gallons a day, 1928-29

\begin{tabular}{|c|c|c|c|c|c|c|c|c|c|c|c|c|}
\hline Day & July & Aug. & Sept. & Oct. & Nor. & Dec. & Jan. & Feb. & Mar. & Apr. & May & June \\
\hline$\ldots$ & 7.5 & $\begin{array}{l}16.8 \\
10.7 \\
11.4 \\
10.2 \\
10.2\end{array}$ & $\begin{array}{l}6.9 \\
36 \\
22 \\
16.6 \\
12.4\end{array}$ & $\begin{array}{r}4.4 \\
16.7 \\
5.8 \\
5.2 \\
6.9\end{array}$ & $\begin{array}{l}4.4 \\
4.4 \\
4.0 \\
4.1 \\
5.9\end{array}$ & $\begin{array}{r}4.9 \\
4.6 \\
4.5 \\
4.4 \\
70\end{array}$ & $\begin{array}{c}14.7 \\
8.0 \\
46 \\
11.6 \\
9.4\end{array}$ & $\begin{array}{l}5.4 \\
5.3 \\
5.2 \\
4.9 \\
4.7\end{array}$ & $\begin{array}{l}56 \\
31 \\
28 \\
56 \\
71\end{array}$ & $\begin{array}{l}3.9 \\
4.1 \\
4.0 \\
3.6 \\
3.4\end{array}$ & $\begin{array}{l}3.7 \\
3.3 \\
3.2 \\
3.1 \\
\text { 3. }\end{array}$ & $\begin{array}{r}3 . \\
91 \\
21\end{array}$ \\
\hline $\begin{array}{l}6 \\
7 \\
8 \\
8\end{array}$ & $\begin{array}{l}15 \\
10.3\end{array}$ & $\begin{array}{r}7.9 \\
7.5 \\
7.0 \\
6.7 \\
12.8\end{array}$ & $\begin{array}{r}9.0 \\
14.7 \\
9.6 \\
12.7 \\
48\end{array}$ & 4.7 & $\begin{array}{l}4.5 \\
66 \\
11.2 \\
27 \\
67\end{array}$ & $\begin{array}{l}75 \\
19.6 \\
18.5 \\
40 \\
34\end{array}$ & $\begin{array}{r}21 \\
9.6 \\
10.1 \\
7.5 \\
6.8\end{array}$ & $\begin{array}{l}7.3 \\
7.5 \\
5.2 \\
4.6 \\
4.5\end{array}$ & $\begin{array}{r}41 \\
101 \\
70 \\
97 \\
42\end{array}$ & $\begin{array}{r}3.5 \\
4.4 \\
8.6 \\
3.9 \\
69\end{array}$ & $\begin{array}{l}4.4 \\
4.2 \\
5.0 \\
3.4 \\
4.0\end{array}$ & 9 \\
\hline (1) & $\begin{array}{r}10.3 \\
7.7 \\
8.5 \\
16.3 \\
20\end{array}$ & $\begin{array}{l}9.0 \\
9.3 \\
8.9 \\
7.2 \\
8.5\end{array}$ & $\begin{array}{c}25 \\
9.4 \\
7.5 \\
6.7 \\
6.3\end{array}$ & 4.1 & $\begin{array}{r}172 \\
33 \\
30 \\
40 \\
26\end{array}$ & $\begin{array}{r}28 \\
50 \\
61 \\
101 \\
46\end{array}$ & $\begin{array}{r}6.3 \\
6.0 \\
6.2 \\
37 \\
11.0\end{array}$ & \begin{tabular}{|l|l}
4.5 \\
34 \\
7.8 \\
53 \\
103
\end{tabular} & $\begin{array}{l}43 \\
14.6 \\
11.2 \\
11.9 \\
27\end{array}$ & $\begin{array}{c}154 \\
208 \\
37 \\
11.4 \\
11.6\end{array}$ & $\begin{array}{r}11.4 \\
9.4 \\
39 \\
17.0 \\
8.2\end{array}$ & $\begin{array}{r}5 . \\
4 . \\
7 . \\
4 . \\
20\end{array}$ \\
\hline 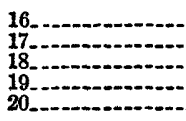 & $\begin{array}{l}50 \\
26 \\
14.4 \\
12.2 \\
8.8\end{array}$ & $\begin{array}{l}25 \\
45 \\
18.4 \\
9.2 \\
13.2\end{array}$ & $\begin{array}{r}6.8 \\
.6 .2 \\
6.5 \\
35 \\
7.5\end{array}$ & $\begin{array}{l}3.7 \\
3.7 \\
3.7 \\
3.5\end{array}$ & $\begin{array}{r}10.4 \\
8.8 \\
14.4 \\
11.3 \\
54\end{array}$ & \begin{tabular}{r|}
118 \\
15.0 \\
17.1 \\
12.3 \\
9.6
\end{tabular} & $\begin{array}{r}8.1 \\
6.7 \\
7.0 \\
19.2 \\
13.0\end{array}$ & $\begin{array}{r}185 \\
347 \\
158 \\
43 \\
168\end{array}$ & $\begin{array}{l}9.6 \\
8.1 \\
7.3 \\
6.9 \\
6.5\end{array}$ & $\begin{array}{r}7.9 \\
6.7 \\
21 \\
6.0 \\
5.2\end{array}$ & $\begin{array}{l}5.3 \\
4.7 \\
4.3 \\
4.0 \\
3.9\end{array}$ & $\begin{array}{l}6 . \\
4 . \\
4 . \\
3 . \\
3 .\end{array}$ \\
\hline (1) & $\begin{array}{l}7.5 \\
20 \\
12.3 \\
57 \\
83\end{array}$ & $\begin{array}{r}8.4 \\
11.8 \\
18.9 \\
16.5 \\
9.0\end{array}$ & $\begin{array}{l}6.5 \\
5.9 \\
5.4 \\
5.4 \\
5.2\end{array}$ & $\begin{array}{l}3.8 \\
4.7 \\
3.5 \\
3.3 \\
3.2\end{array}$ & $\begin{array}{r}27 \\
9.8 \\
8.5 \\
7.5 \\
6.7\end{array}$ & $\begin{array}{r}9.0 \\
8.1 \\
7.4 \\
8.6 \\
56\end{array}$ & $\begin{array}{c}9.6 \\
278 \\
55 \\
12.8 \\
9.6\end{array}$ & \begin{tabular}{|c|}
135 \\
88 \\
22 \\
17.4 \\
9.8
\end{tabular} & $\begin{array}{l}6.0 \\
5.6 \\
5.3\end{array}$ & $\begin{array}{l}4.8 \\
4.6 \\
4.6 \\
4.7 \\
4.1\end{array}$ & $\begin{array}{l}3.7 \\
3.8 \\
5.9 \\
6.2 \\
3.9\end{array}$ & 3. \\
\hline - & $\begin{array}{l}230 \\
79 \\
16.4 \\
12.2 \\
10.1 \\
11.7\end{array}$ & $\begin{array}{r}7.7 \\
8.2 \\
10.4 \\
6.5 \\
6.0 \\
5.9\end{array}$ & $\begin{array}{l}4.9 \\
4.5 \\
5.0 \\
8.5 \\
5.9\end{array}$ & $\begin{array}{c}3.0 \\
23 \\
23 \\
4.7 \\
6.9 \\
5.2\end{array}$ & $\begin{array}{l}6.2 \\
5.9 \\
5.7 \\
5.4 \\
5.6\end{array}$ & $\begin{array}{l}45 \\
11.3 \\
19.2 \\
26 \\
11.0 \\
8.8\end{array}$ & $\begin{array}{l}7.9 \\
7.2 \\
7.2 \\
6.7 \\
6.0 \\
5.9\end{array}$ & \begin{tabular}{|c|}
15.3 \\
33 \\
126 \\
2. \\
\end{tabular} & $\begin{array}{l}4.7 \\
4.4 \\
4.2 \\
4.1 \\
3.9 \\
3.9\end{array}$ & $\begin{array}{l}4.1 \\
3.9 \\
3.7 \\
3.5 \\
3.6\end{array}$ & $\begin{array}{l}3.7 \\
8.4 \\
4.9 \\
4.1 \\
3.8 \\
3.7\end{array}$ & 2 \\
\hline
\end{tabular}

\begin{tabular}{|c|c|c|c|c|c|c|}
\hline \multirow{2}{*}{ Month } & \multicolumn{3}{|c|}{ Million gallons a day } & \multirow{2}{*}{$\begin{array}{l}\text { Second- } \\
\text { foet } \\
\text { (mean) }\end{array}$} & \multicolumn{2}{|c|}{ Total run-off } \\
\hline & Maximum & Minimam & Mean & & $\begin{array}{l}\text { Millon } \\
\text { gallons }\end{array}$ & Acre-feet \\
\hline $\begin{array}{l}\text { July } \\
\text { August } \\
\text { September } \\
\text { October- } \\
\text { November.- } \\
\text { December } \\
\text { January } \\
\text { February } \\
\text { March } \\
\text { April } \\
\text { May } \\
\text { June }\end{array}$ & $\begin{array}{r}230 \\
45 \\
48 \\
23 \\
172 \\
118 \\
278 \\
347 \\
101 \\
1208 \\
39 \\
21\end{array}$ & $\begin{array}{l}5.9 \\
4.5 \\
4.0 \\
4.4 \\
5.9 \\
4.5 \\
3.9 \\
3.4 \\
3.1 \\
2.9\end{array}$ & $\begin{array}{c}29.0 \\
11.7 \\
12.1 \\
6.00 \\
22.9 \\
30.4 \\
21.6 \\
57.3 \\
25.5 \\
20.6 \\
6.35 \\
5.55\end{array}$ & $\begin{array}{c}44.9 \\
18.1 \\
18.7 \\
9.28 \\
35.4 \\
47.0 \\
33.4 \\
88.7 \\
39.5 \\
31.9 \\
9.82 \\
8.69\end{array}$ & $\begin{array}{r}899 \\
364 \\
362 \\
186 \\
687 \\
944 \\
671 \\
1,600 \\
791 \\
619 \\
197 \\
166\end{array}$ & $\begin{array}{r}2,760 \\
1,110 \\
1,110 \\
5771 \\
2,110 \\
2,800 \\
2,050 \\
4,920 \\
2,430 \\
1,900 \\
604 \\
511\end{array}$ \\
\hline The year-- & 347 & 2.9 & 20.5 & 31.7 & 7,490 & 23,000 \\
\hline
\end{tabular}


EAST WAIHUAIKI STREAM NEAR KEANAE, MAUI

Location.-Water-stage recorder 1,000 feet above Koolau ditch crossing and trail and 3 miles southeast of Keanae post office.

Drainage area. - 3.7 square miles.

ReCORDS AVAILABLw.-December, 1913, to October, 1917; July, 1922, to June, 1929.

Extremes.-Maximum discharge during year, 976 million gallons a day or 1,510 second-feet Feb. 17 (gage height, 7.26 feet); minimum, 2.8 million gallons a day or 4.3 second-feet June 29,30 .

1913-1917, 1922-1929: Maximum discharge, 1,900 million gallons a day or 2,940 second-feet Jan. 18, 1916 (gage height, 8.35 feet); minimum, 1.0 million gallons a day or 1.6 second-feet Oct. 22, 23, 1917; Aug. 1, 2, 1922.

Flood of Dec. 24, 1921, may have reached a higher stage, but owing to destruction of station no data are available for this peak.

REMARKs.-Records good for ordinary stages, fair for estimated periods, and poor for high stages. No diversions.

Discharge, in million gallons a day, 1928-29

\begin{tabular}{|c|c|c|c|c|c|c|c|c|c|c|c|c|}
\hline Day & July & Aug. & Sept. & Oct. & Nov. & Dec. & Jan. & Feb. & Mar. & Apr. & May & June \\
\hline & $\begin{array}{r}80 \\
11.1 \\
9.1 \\
7.9 \\
6.5\end{array}$ & $\begin{array}{l}16.6 \\
10.8 \\
12.2 \\
11.0 \\
10.7\end{array}$ & \begin{tabular}{l}
\multicolumn{1}{c}{7.6} \\
44 \\
26 \\
18.2 \\
12.2
\end{tabular} & $\begin{array}{r}11.5 \\
10.7 \\
5.9 \\
6.5 \\
9.1\end{array}$ & $\begin{array}{l}4.6 \\
4.3 \\
3.9 \\
4.2 \\
7.4\end{array}$ & $\begin{array}{r}4.9 \\
4.7 \\
4.4 \\
4.3 \\
98\end{array}$ & $\begin{array}{l}16.6 \\
9.7 \\
56 \\
13 \\
10.6\end{array}$ & $\begin{array}{l}5.6 \\
5.4 \\
5.1 \\
4.9 \\
4.7\end{array}$ & $\begin{array}{l}29 \\
63 \\
83\end{array}$ & $\begin{array}{l}5.2 \\
5.3 \\
5.4 \\
4.7 \\
4.4\end{array}$ & $\begin{array}{l}4.0 \\
3.6 \\
3.5 \\
3.3 \\
3.6\end{array}$ & $\begin{array}{l}3 . \\
10 . \\
21\end{array}$ \\
\hline & $\begin{array}{r}5.7 \\
17.2 \\
19.5 \\
9.4 \\
10.0\end{array}$ & $\begin{array}{r}8.5 \\
7.9 \\
7.2 \\
6.9 \\
14.2\end{array}$ & $\begin{array}{r}9.1 \\
18.5 \\
10.5 \\
14.5 \\
59\end{array}$ & $\begin{array}{l}6.1 \\
5.3 \\
5.1 \\
4.6 \\
4.3\end{array}$ & \begin{tabular}{l|}
4.7 \\
80 \\
12.8 \\
31 \\
58
\end{tabular} & $\begin{array}{l}71 \\
18.1 \\
17.8 \\
39 \\
35\end{array}$ & $\begin{array}{r}25 \\
11.1 \\
11.2 \\
9.1 \\
7.9\end{array}$ & $\begin{array}{l}7.5 \\
8.8 \\
5.4 \\
4.7 \\
4.5\end{array}$ & $\begin{array}{r}32 \\
88 \\
65 \\
111 \\
42\end{array}$ & \begin{tabular}{|r|}
4.6 \\
6.4 \\
11.9 \\
5.4 \\
112
\end{tabular} & $\begin{array}{l}5.2 \\
5.0 \\
6.1 \\
3.8 \\
4.4\end{array}$ & $\begin{array}{l}9 . \\
5 . \\
4 . \\
3 . \\
3 .\end{array}$ \\
\hline & $\begin{array}{r}10.3 \\
7.6 \\
7.9 \\
15.8 \\
14.7\end{array}$ & $\begin{array}{l}9.8 \\
9.7 \\
9.0 \\
7.7 \\
9.3\end{array}$ & $\begin{array}{l}28 \\
10.4 \\
8.5 \\
7.2 \\
6.8\end{array}$ & $\begin{array}{l}4.1 \\
4.0 \\
3.8 \\
5.2 \\
6.4\end{array}$ & $\begin{array}{r}170 \\
27 \\
28 \\
45 \\
23\end{array}$ & $\begin{array}{r}26 \\
54 \\
67 \\
112 \\
49\end{array}$ & $\begin{array}{c}7.6 \\
7.1 \\
7.5 \\
33 \\
13.0\end{array}$ & $\begin{array}{c}4.7 \\
35 \\
8.6 \\
56 \\
100\end{array}$ & $\begin{array}{l}48 \\
16.0 \\
12.6 \\
13.1 \\
27\end{array}$ & $\begin{array}{c}158 \\
225 \\
33 \\
13.6 \\
13.9\end{array}$ & $\begin{array}{c}14.9 \\
11.3 \\
44.1 \\
19.1 \\
9.0\end{array}$ & $\begin{array}{r}5 . \\
7 . \\
54 \\
24\end{array}$ \\
\hline & $\begin{array}{l}50 \\
23 \\
12.6 \\
11.8 \\
8.5\end{array}$ & $\begin{array}{l}29 \\
48 \\
18.0 \\
9.1 \\
13.4\end{array}$ & $\begin{array}{r}7.2 \\
6.4 \\
6.3 \\
42 \\
7.6\end{array}$ & $\begin{array}{l}4.3 \\
4.1 \\
3.9 \\
4.0 \\
3.7\end{array}$ & $\begin{array}{c}11.1 \\
9.1 \\
14.3 \\
10.8 \\
57\end{array}$ & $\begin{array}{r}105 \\
15.9 \\
18.3 \\
13.3 \\
11.5\end{array}$ & $\begin{array}{c}9.1 \\
7.6 \\
8.2 \\
20 \\
13.9\end{array}$ & $\begin{array}{r}170 \\
358 \\
140 \\
45 \\
165\end{array}$ & $\begin{array}{r}11.1 \\
9.8 \\
9.1 \\
8.5 \\
7.9\end{array}$ & $\begin{array}{r}9.0 \\
7.9 \\
24 \\
7.6 \\
6.4\end{array}$ & $\begin{array}{l}6.0 \\
5.1 \\
4.5 \\
4.2 \\
4.0\end{array}$ & $\begin{array}{l}7 . \\
5 . \\
4 . \\
4 . \\
3 .\end{array}$ \\
\hline - & $\begin{array}{l}7.5 \\
23 \\
13.0 \\
45 \\
87\end{array}$ & $\begin{array}{r}9.3 \\
12.1 \\
21 \\
18.2 \\
9.8\end{array}$ & $\begin{array}{l}6.4 \\
5.8 \\
5.4 \\
5.4 \\
5.0\end{array}$ & $\begin{array}{l}4.3 \\
5.4 \\
4.0 \\
3.8 \\
3.7\end{array}$ & \begin{tabular}{c|}
28 \\
9.8 \\
8.5 \\
7.8 \\
6.7
\end{tabular} & $\begin{array}{r}10.4 \\
9.1 \\
8.5 \\
10.2 \\
87\end{array}$ & $\begin{array}{c}10.4 \\
242 \\
56 \\
13.9 \\
9.8\end{array}$ & \begin{tabular}{|c|}
124 \\
88 \\
20 \\
16.2 \\
10.4
\end{tabular} & $\begin{array}{l}7.6 \\
7.1 \\
6.8 \\
6.6 \\
6.3\end{array}$ & $\begin{array}{l}5.7 \\
5.4 \\
5.3 \\
5.4 \\
4.7\end{array}$ & \begin{tabular}{l|}
3.8 \\
4.0 \\
7.0 \\
6.9 \\
4.2
\end{tabular} & $\begin{array}{l}\text { 3. } \\
3 . \\
3 .\end{array}$ \\
\hline . & $\begin{array}{l}215 \\
64 \\
15.1 \\
11.8 \\
9.8 \\
11.0\end{array}$ & $\begin{array}{r}7.9 \\
8.3 \\
11.0 \\
7.0 \\
6.5 \\
6.3\end{array}$ & $\begin{array}{r}4.7 \\
4.7 \\
5.6 \\
10.6 \\
5.4\end{array}$ & $\begin{array}{r}3.5 \\
25 \\
19.2 \\
4.9 \\
8.4 \\
6.0\end{array}$ & $\begin{array}{l}6.2 \\
5.7 \\
5.5 \\
5.4 \\
5.7\end{array}$ & $\begin{array}{l}38 \\
12.6 \\
20 \\
25 \\
11.8 \\
9.8\end{array}$ & $\begin{array}{l}8.5 \\
7.7 \\
7.7 \\
7.0 \\
6.3 \\
5.9\end{array}$ & $\begin{array}{r}24 \\
49 \\
115\end{array}$ & $\begin{array}{l}6.0 \\
5.8 \\
5.5 \\
5.4 \\
5.1 \\
6.1\end{array}$ & $\begin{array}{l}4.6 \\
4.3 \\
4.1 \\
4.0 \\
4.0\end{array}$ & $\begin{array}{l}3.8 \\
7.5 \\
5.7 \\
4.2 \\
3.8 \\
3.5\end{array}$ & $\begin{array}{l}3 . \\
3 . \\
3 . \\
2 . \\
3 .\end{array}$ \\
\hline \multirow{2}{*}{\multicolumn{4}{|c|}{ Month }} & \multicolumn{4}{|c|}{ Million gallons a day } & \multirow{2}{*}{\multicolumn{2}{|c|}{$\begin{array}{l}\text { Second- } \\
\text { feet } \\
\text { (mean) }\end{array}$}} & \multicolumn{3}{|c|}{ Total run-of } \\
\hline & & & & aximum & \multicolumn{2}{|c|}{ Minimum } & Mean & & & $\begin{array}{l}\text { Million } \\
\text { gallons }\end{array}$ & \multicolumn{2}{|c|}{ Acre-feet } \\
\hline \multicolumn{4}{|c|}{ 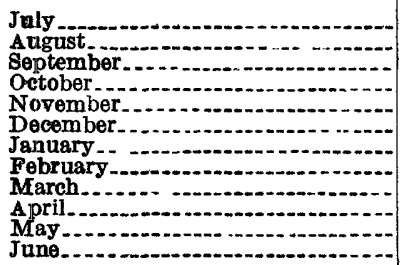 } & $\begin{array}{r}215 \\
48 \\
59 \\
25 \\
170 \\
112 \\
242 \\
358 \\
111 \\
225 \\
44 \\
24\end{array}$ & & $\begin{array}{l}5.7 \\
6.3 \\
4.7 \\
3.5 \\
3.9 \\
4.3 \\
5.9 \\
4.5 \\
5.1 \\
4.0 \\
3.3 \\
2.9\end{array}$ & $\begin{aligned} 27.1 \\
12.5 \\
13.6 \\
6.4 \\
23.2 \\
32.6 \\
21.7 \\
56.6 \\
26.3 \\
23.7 \\
7.0 \\
5.7\end{aligned}$ & & & $\begin{array}{r}841 \\
386 \\
409 \\
201 \\
696 \\
1,010 \\
673 \\
1,590 \\
816 \\
711 \\
219 \\
173\end{array}$ & & $\begin{array}{r}2,580 \\
1,190 \\
1,250 \\
616 \\
2,140 \\
3,100 \\
2,060 \\
4,860 \\
2,500 \\
2,180 \\
672 \\
530\end{array}$ \\
\hline & 358 & & 2.9 & 21 . & & 32.8 & 7,720 & & 23,70 \\
\hline
\end{tabular}




\section{WEST WAILUAIKI STREAM NEAR KEANAE, MAUI}

Locatron.-Water-stage recorder 500 feet above Koolau ditch crossing and trail bridge and $23 / 4$ miles south of Keanae post office.

Drain age area.- 3.6 square miles.

RecoRds AvaIlABle.-January, 1914, to October, 1917; November, 1921, to June, 1929.

EXTREMES.-Maximum discharge during year, 2,210 million gallons a day or 3,420 second-feet Feb. 17 (gage height, 9.57 feet); minimum, 1.7 million gallons a day or 2.6 second-feet June 29,30 .

1914-1917, 1921-1929: Maximum discharge (estimated), 4,500 million gallons a day or 6,960 second-feet Jan. 14, 1923 (gage height, from floodmarks, about 13.5 feet); minimum, 0.3 million gallons a day or 0.45 secondfoot July 26,1922 .

REMaRKs.-Records good for ordinary stages; poor for extremely high stages. No diversions.

Discharge, in million gallons a day, 1928-29

\begin{tabular}{|c|c|c|c|c|c|c|c|c|c|c|c|c|}
\hline Day & July & Aug. & Sept. & Oct. & Nov. & Dec. & Jan. & Feb. & Mar. & Apr. & May & June \\
\hline $\begin{array}{l}2 \\
3 \\
4 \\
5\end{array}$ & $\begin{array}{l}98 \\
16.0 \\
13.1 \\
10.5 \\
7.9\end{array}$ & $\begin{array}{l}22 \\
13.8 \\
15.3 \\
13.8 \\
13.3\end{array}$ & $\begin{array}{l}9.5 \\
53 \\
35 \\
28 \\
18.0\end{array}$ & $\begin{array}{r}16.9 \\
15.2 \\
7.0 \\
7.7 \\
10.7\end{array}$ & $\begin{array}{r}5.4 \\
4.7 \\
4.0 \\
4.6 \\
10.2\end{array}$ & $\begin{array}{r}4.6 \\
4.0 \\
3.7 \\
3.7 \\
135\end{array}$ & $\begin{array}{l}19.2 \\
29 \\
59 \\
16.0 \\
13.7\end{array}$ & $\begin{array}{l}5.1 \\
4.9 \\
4.4 \\
4.2 \\
3.7\end{array}$ & $\begin{array}{r}60 \\
35 \\
36 \\
69 \\
103\end{array}$ & $\begin{array}{l}3.2 \\
3.3 \\
3.2 \\
2.7 \\
2.2\end{array}$ & $\begin{array}{l}2.9 \\
2.5 \\
2.2 \\
2.0 \\
2.6\end{array}$ & $\begin{array}{r}5.0 \\
2.8 \\
2.8 \\
18.6 \\
34\end{array}$ \\
\hline $\begin{array}{l}6 \\
70 \\
9\end{array}$ & \begin{tabular}{l|l} 
& 6.8 \\
26 & \\
28 & \\
13.8 & \\
13.3 &
\end{tabular} & $\begin{array}{r}10.1 \\
9.1 \\
7.7 \\
7.1 \\
21\end{array}$ & $\begin{array}{l}12.8 \\
35 \\
16.0 \\
17.1 \\
73\end{array}$ & $\begin{array}{l}6.8 \\
5.9 \\
5.4 \\
4.6 \\
4.2\end{array}$ & $\begin{array}{l}5.4 \\
97 \\
20 \\
60 \\
86\end{array}$ & $\begin{array}{l}93 \\
28 \\
24 \\
52 \\
46\end{array}$ & $\begin{array}{l}30 \\
13.5 \\
14.0 \\
10.5 \\
8.5\end{array}$ & $\begin{array}{r}6.6 \\
10.0 \\
5.0 \\
4.0 \\
3.6\end{array}$ & $\begin{array}{r}50 \\
102 \\
82 \\
220 \\
55\end{array}$ & $\begin{array}{r}2.5 \\
4.2 \\
13.3 \\
4.6 \\
138\end{array}$ & $\begin{array}{l}5.4 \\
5.6 \\
7.3 \\
4.2 \\
5.0\end{array}$ & \\
\hline 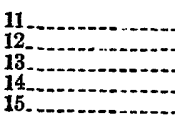 & $\begin{array}{l}13.5 \\
10.3 \\
12.6 \\
31 \\
23\end{array}$ & $\begin{array}{r}14.2 \\
11.5 \\
11.3 \\
9.1 \\
11.6\end{array}$ & $\begin{array}{r}40 \\
14.2 \\
10.7 \\
8.9 \\
7.9\end{array}$ & $\begin{array}{l}3.9 \\
3.5 \\
2.8 \\
4.4 \\
5.9\end{array}$ & $\begin{array}{r}234 \\
44 \\
39 \\
54 \\
34\end{array}$ & $\begin{array}{r}37 \\
67 \\
92 \\
148 \\
53\end{array}$ & $\begin{array}{r}7.5 \\
6.7 \\
7.7 \\
44 \\
18.0\end{array}$ & $\begin{array}{c}3.8 \\
38 \\
10.7 \\
53 \\
112\end{array}$ & $\begin{array}{l}63 \\
20 \\
14.8 \\
17.8 \\
27\end{array}$ & $\begin{array}{l}212 \\
319 \\
51 \\
19.8 \\
20\end{array}$ & $\begin{array}{l}19.2 \\
14.2 \\
57 \\
25 \\
12.6\end{array}$ & 31 \\
\hline 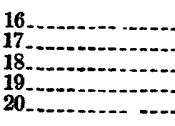 & $\begin{array}{l}70 \\
35 \\
19.0 \\
15.5 \\
12.0\end{array}$ & $\begin{array}{l}34 \\
60 \\
27 \\
12.8 \\
16.1\end{array}$ & $\begin{array}{r}8.7 \\
7.0 \\
6.8 \\
50 \\
10.3\end{array}$ & $\begin{array}{l}3.5 \\
3.0 \\
2.8 \\
2.9 \\
2.4\end{array}$ & $\begin{array}{l}15.8 \\
12.4 \\
22 \\
15.0 \\
79\end{array}$ & $\begin{array}{l}178 \\
20 \\
22 \\
17.4 \\
14.5\end{array}$ & $\begin{array}{r}10.9 \\
7.9 \\
9.5 \\
29 \\
18.0\end{array}$ & \begin{tabular}{|r|}
358 \\
778 \\
240 \\
55 \\
267
\end{tabular} & $\begin{array}{r}12.0 \\
9.9 \\
8.3 \\
7.3 \\
6.7\end{array}$ & $\begin{array}{r}12.2 \\
9.7 \\
27 \\
9.5 \\
7.3\end{array}$ & $\begin{array}{l}7.5 \\
5.9 \\
5.0 \\
4.3 \\
3.9\end{array}$ & 3. \\
\hline $\begin{array}{l}23 \\
24 \\
25\end{array}$ & \begin{tabular}{|c|}
9.5 \\
30 \\
19.0 \\
81 \\
124 \\
\end{tabular} & $\begin{array}{l}12.4 \\
13.8 \\
24 \\
28 \\
13.8\end{array}$ & $\begin{array}{l}7.7 \\
6.5 \\
5.7 \\
5.6 \\
4.9\end{array}$ & $\begin{array}{l}3.5 \\
5.1 \\
3.0 \\
2.6 \\
2.5\end{array}$ & $\begin{array}{r}34 \\
13.8 \\
11.1 \\
9.3 \\
7.5\end{array}$ & $\begin{array}{r}12.4 \\
10.7 \\
9.1 \\
10.9 \\
120\end{array}$ & $\begin{array}{l}13.1 \\
436 \\
71 \\
20 \\
13.1\end{array}$ & \begin{tabular}{|c|}
168 \\
109 \\
31 \\
30 \\
13.8
\end{tabular} & $\begin{array}{l}6.0 \\
5.4 \\
5.0 \\
4.7 \\
4.3\end{array}$ & $\begin{array}{l}6.2 \\
5.7 \\
5.4 \\
5.3 \\
4.3\end{array}$ & 3.6 & $\begin{array}{l}3 . \\
2 . \\
2 . \\
2 .\end{array}$ \\
\hline $\begin{array}{l}26 \\
28 \\
30\end{array}$ & \begin{tabular}{|l|}
393 \\
107 \\
24 \\
16.0 \\
13.8 \\
15.0
\end{tabular} & $\begin{array}{r}10.5 \\
9.5 \\
12.3 \\
7.9 \\
6.7 \\
6.5\end{array}$ & $\begin{array}{r}4.4 \\
4.4 \\
8.1 \\
17.1 \\
6.5\end{array}$ & $\begin{array}{c}2.1 \\
30 \\
20 \\
5.6 \\
13.2 \\
8.0\end{array}$ & $\begin{array}{l}6.7 \\
6.0 \\
5.4 \\
5.1 \\
5.4\end{array}$ & \begin{tabular}{l|}
38 \\
18.0 \\
29 \\
25 \\
13.8 \\
11.7
\end{tabular} & $\begin{array}{l}10.6 \\
.8 .9 \\
8.3 \\
7.0 \\
6.2 \\
5.6\end{array}$ & \begin{tabular}{|c}
33 \\
58 \\
159 \\
\hdashline \\
\hdashline- \\
\hdashline-9 \\
\end{tabular} & $\begin{array}{l}4.2 \\
3.9 \\
3.7 \\
3.5 \\
3.2 \\
2.9\end{array}$ & $\begin{array}{l}4.2 \\
3.9 \\
3.3 \\
3.2 \\
3.2\end{array}$ & $\begin{array}{r}3.6 \\
11.1 \\
7.8 \\
5.0 \\
4.0 \\
3.5\end{array}$ & $\begin{array}{l}2 . \\
2 . \\
1 . \\
1 . \\
2 .\end{array}$ \\
\hline
\end{tabular}

\begin{tabular}{|c|c|c|c|c|c|c|}
\hline \multirow{2}{*}{ Month } & \multicolumn{3}{|c|}{ Million gallons a day } & \multirow{2}{*}{$\begin{array}{c}\text { Second- } \\
\text { feet } \\
\text { (mean) }\end{array}$} & \multicolumn{2}{|c|}{ Total run-off } \\
\hline & Maximum & Minimum & Mean & & $\begin{array}{l}\text { Million } \\
\text { gallons }\end{array}$ & Acre-feet \\
\hline 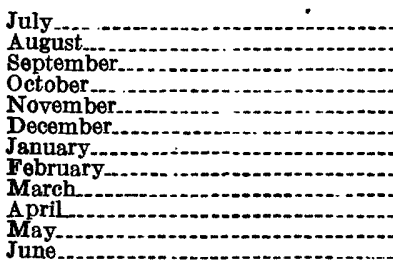 & $\begin{array}{r}393 \\
60 \\
73 \\
30 \\
234 \\
178 \\
436 \\
778 \\
220 \\
319 \\
57 \\
34\end{array}$ & $\begin{array}{l}6.8 \\
6.5 \\
4.4 \\
2.1 \\
4.0 \\
3.7 \\
5.6 \\
3.6 \\
2.9 \\
2.2 \\
2.0 \\
1.7\end{array}$ & $\begin{array}{l}42.2 \\
15.7 \\
17.8 \\
66.94 \\
31.4 \\
43.3 \\
31.4 \\
91.7 \\
33.7 \\
30.3 \\
8.33 \\
6.97\end{array}$ & $\begin{array}{r}65.3 \\
24.3 \\
27.5 \\
10.7 \\
48.6 \\
67.0 \\
48.6 \\
142 . \\
52.1 \\
46.9 \\
12.9 \\
10.8\end{array}$ & $\begin{array}{r}1,310 \\
486 \\
533 \\
215 \\
941 \\
1,340 \\
972 \\
2,570 \\
1,050 \\
909 \\
258 \\
200\end{array}$ & $\begin{array}{l}4,010 \\
1,490 \\
1,640 \\
660 \\
2,890 \\
4,120 \\
2,990 \\
7,880 \\
3,210 \\
2,790 \\
792 \\
642\end{array}$ \\
\hline The year. & 778 & 1.7 & 29.6 & 45.8 & 10,800 & 33,100 \\
\hline
\end{tabular}


EAST WATIUANUI STREAM NEAR KEANAE, MAUI

Location.-Water-stage recorder 125 feet above Koolau ditch intake, 250 feet above trail, and $2 \frac{1}{2}$ miles south of Keanae post office.

Drainage AREA. - 0.5 square mile.

Reconds avaIlable.- January, 1914, to October, 1917; November, 1921, to June, 1929.

EXTrEmes.-Maximum discharge during year, 633 million gallons a day or 979 second-feet Feb. 17 (gage height, 4.89 feet); minimum, 0.6 million gallons a day or 0.9 second-foot June 29 .

1914-1917, 1921-1929: Maximum discharge, 1,050 million gallons a day or 1,620 second-feet Feb. 12, 1925 (gage height, 6.96 feet); minimum, 0.1 million gallons a day or 0.2 second-foot Apr. 11, 1926 .

Remarks. - Records fair for ordinary stages; poor for estimated periods and high stages. No diversions.

Discharge, in million gallons a day, 1928-29

\begin{tabular}{|c|c|c|c|c|c|c|c|c|c|c|c|c|}
\hline Day & July & Aug. & Sept. & Oct. & Nov. & Dec. & Jan. & Feb. & Mar. & Apr. & May & June \\
\hline & 2.8 & $\begin{array}{l}5.9 \\
3.9 \\
4.9 \\
4.4 \\
3.9\end{array}$ & $\begin{array}{r}2.3 \\
20 \\
12.4 \\
5.8 \\
4.5\end{array}$ & $\begin{array}{l}8.1 \\
4.5 \\
1.9 \\
2.7 \\
3.6\end{array}$ & $\begin{array}{l}1.6 \\
1.4 \\
1.2 \\
1.4 \\
4.0\end{array}$ & $\begin{array}{r}1.2 \\
1.0 \\
1.0 \\
28\end{array}$ & $\begin{array}{l}2.6 \\
8.6 \\
3.8 \\
2.8\end{array}$ & $\begin{array}{l}1.3 \\
1.2 \\
1.1 \\
1.1 \\
1.0\end{array}$ & $\begin{array}{r}15.2 \\
8.9 \\
12.0 \\
23 \\
40\end{array}$ & $\begin{array}{r}1.1 \\
1.2 \\
1.2 \\
.9 \\
.8\end{array}$ & $\begin{array}{r}1.1 \\
.9 \\
.8 \\
.8 \\
1.0\end{array}$ & $\begin{array}{r}0.9 \\
.9 \\
.9 \\
2.1 \\
3.3\end{array}$ \\
\hline $\begin{array}{c}6 \\
7 \ldots \\
8 \cdots \\
90 \\
10\end{array}$ & 3.8 & $\begin{array}{l}2.6 \\
2.4 \\
2.1 \\
2.1 \\
5.3\end{array}$ & $\begin{array}{r}3.1 \\
7.2 \\
3.4 \\
8.1 \\
31\end{array}$ & $\begin{array}{l}2.1 \\
1.8 \\
1.8 \\
1.4 \\
1.3\end{array}$ & $\begin{array}{r}1.7 \\
25 \\
5.2 \\
4.4 \\
5.4\end{array}$ & $\begin{array}{r}13.2 \\
3.0 \\
5.2 \\
5.6 \\
6.8\end{array}$ & $\begin{array}{l}7.1 \\
3.1 \\
4.0 \\
2.4 \\
2.1\end{array}$ & $\begin{array}{l}3.3 \\
3.5 \\
1.6 \\
1.3 \\
1.2\end{array}$ & $\begin{array}{l}9.6 \\
14.0 \\
17.9 \\
51 \\
16.2\end{array}$ & $\begin{array}{r}.8 \\
1.6 \\
5.0 \\
1.3 \\
40\end{array}$ & $\begin{array}{l}2.0 \\
2.2 \\
2.1 \\
1.1 \\
1.4\end{array}$ & $\begin{array}{l}1.8 \\
1.2 \\
1.0 \\
.9 \\
1.0\end{array}$ \\
\hline $\begin{array}{l}11 . . . \\
12 \ldots \\
13 \ldots \\
14 \\
15\end{array}$ & 3.0 & $\begin{array}{l}3.6 \\
3.9 \\
3.7 \\
2.6 \\
7.4\end{array}$ & $\begin{array}{l}7.9 \\
3.5 \\
2.6 \\
2.3 \\
2.0\end{array}$ & $\begin{array}{l}1.2 \\
1.1 \\
1.1 \\
2.1 \\
2.0\end{array}$ & $\begin{array}{r}15.9 \\
3.6 \\
7.9 \\
17.4 \\
7.7\end{array}$ & \begin{tabular}{r|r|}
6.9 \\
11.7 \\
17.8 \\
22 \\
8.7
\end{tabular} & $\begin{array}{l}1.9 \\
1.7 \\
1.8 \\
4.0 \\
2.6\end{array}$ & $\begin{array}{l}1.5 \\
12.1 \\
2.1 \\
20 \\
32\end{array}$ & $\begin{array}{r}21 \\
5.3 \\
3.8 \\
4.4 \\
10.8\end{array}$ & $\begin{array}{l}44 \\
40 \\
6.4 \\
3.5 \\
3.0\end{array}$ & $\begin{array}{l}4.2 \\
4.0 \\
8.7 \\
9.0 \\
4.0\end{array}$ & $\frac{1}{8}$ \\
\hline 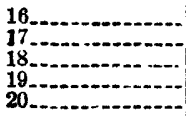 & $\begin{array}{r}13.4 \\
8.1 \\
5.6 \\
5.6 \\
3.5\end{array}$ & $\begin{array}{r}15.0 \\
25 \\
6.7 \\
3.3 \\
16.5\end{array}$ & \begin{tabular}{r|}
2.0 \\
1.8 \\
2.3 \\
16.0 \\
2.1
\end{tabular} & $\begin{array}{l}1.3 \\
1.3 \\
1.2 \\
1.3 \\
1.1\end{array}$ & $\begin{array}{r}3.8 \\
2.6 \\
3.8 \\
3.1 \\
11.4\end{array}$ & $\begin{array}{r}38 \\
4.5 \\
7.3 \\
3.8 \\
3.0\end{array}$ & $\begin{array}{l}1.9 \\
1.6 \\
1.9 \\
2.8 \\
1.9\end{array}$ & $\begin{array}{r}53 \\
103 \\
35 \\
22 \\
31\end{array}$ & $\begin{array}{l}3.3 \\
2.6 \\
2.3 \\
2.0 \\
1.9\end{array}$ & $\begin{array}{r}2.3 \\
2.7 \\
15.1 \\
2.6 \\
2.0\end{array}$ & $\begin{array}{l}2.1 \\
1.8 \\
1.7 \\
1.4 \\
1.3\end{array}$ & $\begin{array}{l}2 . \\
1 . \\
1 . \\
1 . \\
1 .\end{array}$ \\
\hline - & $\begin{array}{r}2.6 \\
9.6 \\
5.3 \\
15.0 \\
23\end{array}$ & $\begin{array}{l}3.3 \\
5.5 \\
7.4 \\
5.8 \\
3.0\end{array}$ & $\begin{array}{l}1.8 \\
1.7 \\
1.6 \\
1.4 \\
1.3\end{array}$ & $\begin{array}{l}1.4 \\
1.9 \\
1.3 \\
1.2 \\
1.2\end{array}$ & $\begin{array}{l}6.0 \\
2.6 \\
2.6 \\
2.3 \\
1.9\end{array}$ & $\begin{array}{r}2.8 \\
2.6 \\
2.3 \\
3.5 \\
33\end{array}$ & $\begin{array}{r}1.9 \\
79 \\
10.3 \\
3.0 \\
2.6\end{array}$ & $\begin{array}{r}17.2 \\
26 \\
5.8 \\
10.7 \\
3.3\end{array}$ & $\begin{array}{l}\text { 1. } \\
1.6 \\
1.4 \\
1.4 \\
1.3\end{array}$ & $\begin{array}{l}1.8 \\
1.7 \\
1.7 \\
1.6 \\
1.4\end{array}$ & $\begin{array}{l}1.2 \\
1.2 \\
2.3 \\
1.9 \\
1.3\end{array}$ & $\begin{array}{l}1.1 \\
1.0 \\
.9 \\
1.1 \\
1.0\end{array}$ \\
\hline $\begin{array}{l}26 \\
28 \\
30 \\
31\end{array}$ & $\begin{array}{r}57 \\
12.1 \\
4.2 \\
3.3 \\
2.8 \\
3.7\end{array}$ & $\begin{array}{l}2.4 \\
3.5 \\
3.9 \\
2.3 \\
1.9 \\
1.9\end{array}$ & $\begin{array}{l}1.2 \\
1.2 \\
1.6 \\
2.6 \\
1.2\end{array}$ & $\begin{array}{l}1.1 \\
8.9 \\
3.6 \\
1.6 \\
1.9 \\
1.6\end{array}$ & $\begin{array}{l}1.8 \\
1.7 \\
1.6 \\
1.4 \\
1.4\end{array}$ & \begin{tabular}{r|r|}
10.3 & 3.3 \\
6.7 \\
11.8 \\
3.5 \\
2.6
\end{tabular} & $\begin{array}{l}2.3 \\
2.0 \\
2.0 \\
1.8 \\
1.6 \\
1.4\end{array}$ & $\begin{array}{r}6.8 \\
10.9 \\
24 \\
\hdashline \\
\hdashline-.\end{array}$ & $\begin{array}{l}1.2 \\
1.2 \\
1.1 \\
1.0 \\
.9 \\
.9\end{array}$ & $\begin{array}{l}1.4 \\
1.3 \\
1.2 \\
1.1 \\
1.1\end{array}$ & $\begin{array}{l}1.1 \\
2.7 \\
1.8 \\
1.3 \\
1.1 \\
1.1\end{array}$ & $\begin{array}{l}.9 \\
.8 \\
.8 \\
.7 \\
.8\end{array}$ \\
\hline
\end{tabular}

\begin{tabular}{|c|c|c|c|c|c|c|}
\hline \multirow{2}{*}{ Month } & \multicolumn{3}{|c|}{ Million gallons a day } & \multirow{2}{*}{$\begin{array}{l}\text { Second- } \\
\text { feet } \\
\text { (mean) }\end{array}$} & \multicolumn{2}{|c|}{ Total run-off } \\
\hline & Maximum & Minimum & Mean & & $\begin{array}{l}\text { Million } \\
\text { gallons }\end{array}$ & Acre-feet \\
\hline $\begin{array}{l}\text { July } \\
\text { August } \\
\text { September.- } \\
\text { October } \\
\text { November- } \\
\text { December } \\
\text { January } \\
\text { February } \\
\text { March } \\
\text { April } \\
\text { May } \\
\text { June }\end{array}$ & $\begin{array}{c}57 \\
25 \\
31 \\
8.9 \\
25 \\
38 \\
79 \\
103 \\
51 \\
44 \\
9.0 \\
8.7 \\
\end{array}$ & $\begin{array}{r}1.9 \\
1.2 \\
1.1 \\
1.2 \\
.9 \\
1.4 \\
1.0 \\
.9 \\
.8 \\
.7\end{array}$ & $\begin{array}{r}7.54 \\
5.36 \\
5.20 \\
2.21 \\
5.06 \\
8.77 \\
5.60 \\
15.5 \\
9.00 \\
6.33 \\
2.21 \\
1.58\end{array}$ & $\begin{array}{c}11.7 \\
8.29 \\
8.05 \\
3.42 \\
7.83 \\
13.6 \\
8.66 \\
24.0 \\
13.9 \\
9.79 \\
3.42 \\
2.44 \\
\end{array}$ & $\begin{array}{l}234 \\
166 \\
156 \\
68.6 \\
152 \\
272 \\
174 \\
433 \\
279 \\
190 \\
68.6 \\
47.5 \\
\end{array}$ & $\begin{array}{r}717 \\
510 \\
479 \\
210 \\
466 \\
834 \\
533 \\
1,330 \\
856 \\
583 \\
210 \\
145 \\
\end{array}$ \\
\hline The year__.... & 103 & .7 & 6.14 & 9.50 & 2,240 & 6,870 \\
\hline
\end{tabular}


WEST WAMUANUI STREAM NEAR KEANAE, MAUI

Locatron.-Water-stage recorder 150 feet above Koolau ditch crossing and intake and $2 \frac{1 / 4}{4}$ miles south of Keanae post office.

Drainage area. - 0.7 square mile.

Records AvaIlable.-December, 1913, to October, 1917; July, 1922, to June, 1929.

Extremes.-Maximum discharge during year, 775 million gallons a day or 1,200 second-feet Feb. 17 (gage height, 5.86 feet); minimum, 0.6 million gallons a day or 0.9 second-foot Apr. 5 .

1914-1917, 1923-1929: Maximum discharge, 1,220 million gallons a day or 1,890 second-feet Jan. 14, 1923 (gage height, 7.70 feet); minimum, 0.2 million gallons a day or 0.3 second-foot July 16-21, 1922 .

REmaRks.-Records fair for ordinary stages; poor for estimated and extremely high stages. No diversions.

Discharge, in million gallons a day, 1928-29

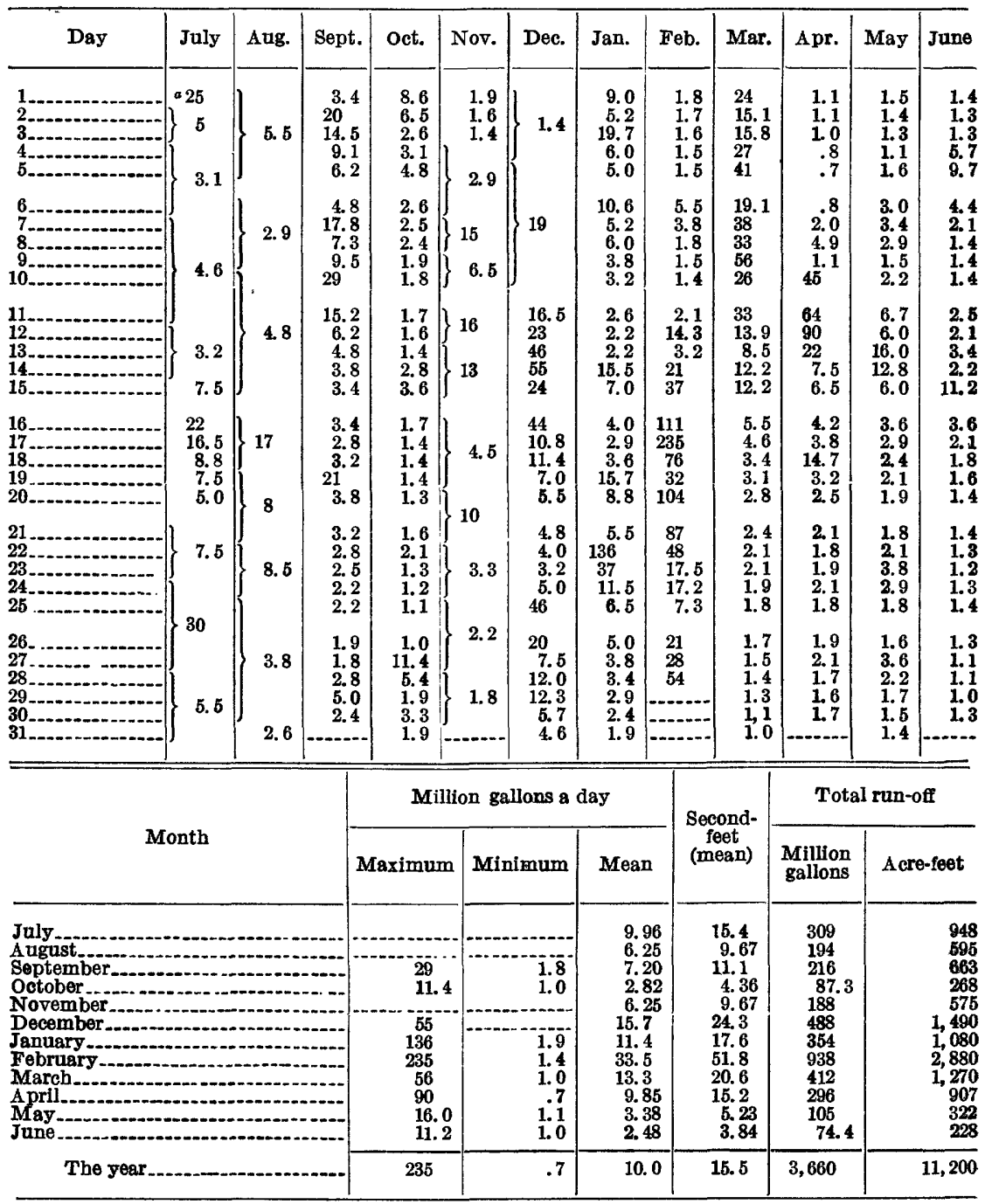

- Estimated. 


\section{KOOLAU DITCH TEAR KEATAE, MAUI}

Location.-Water-stage recorder on west side of Keanae Valley $23 \%$ miles southwest of Keanae post office.

Records AVAIlable.-January, 1910 to December, 1912; and November, 1917, to June, 1929.

Extremes.-Maximum discharge during year, 160 million gallons a day or 248 second-feet Jan. 22 (gage height, 5.50 feet); minimum, 0.3 million gallons a day or 0.5 second-foot Dec. 13.

1910-1912, 1917-1929: Maximum discharge, 175 million gallons a day or 271 second-feet Jan. 4, 1922 (gage height, 6.36 feet); no flow occasionally, when water is shut out of ditch.

REmarks.-Records excellent for ordinary stages and fair for high stages. Discharge Feb. 2 to Mar. 10 from twice daily staff gage readings. Regulated by gates and spillways. Koolau ditch diverts at 1,200-foot elevation from all streams from Makapipi to Alo. No diversions from ditch above station except from several spillways.

Discharge, in million gallons a day, 1928-99

\begin{tabular}{|c|c|c|c|c|c|c|c|c|c|c|c|c|}
\hline & July & Aug. & Sept. & Oct. & Nov. & ec. & Jan. & Feb. & Mar. & Apr. & May & Jun \\
\hline & $\begin{array}{r}111 \\
90 \\
70\end{array}$ & $\begin{array}{l}140 \\
114 \\
1125 \\
118 \\
118\end{array}$ & $\begin{array}{r}73 \\
124 \\
136 \\
140 \\
125\end{array}$ & $\begin{array}{r}61 \\
108 \\
66 \\
69 \\
104\end{array}$ & $\begin{array}{l}46 \\
44 \\
38 \\
37 \\
78\end{array}$ & $\begin{array}{l}50 \\
50 \\
47 \\
44 \\
93\end{array}$ & $\begin{array}{r}95 \\
103 \\
148 \\
133 \\
114\end{array}$ & $\begin{array}{l}54 \\
54 \\
50 \\
47 \\
44\end{array}$ & $\begin{array}{l}148 \\
148 \\
148 \\
148 \\
148\end{array}$ & $\begin{array}{l}44 \\
44 \\
44 \\
38 \\
36\end{array}$ & $\begin{array}{l}36 \\
31 \\
34 \\
34\end{array}$ & \\
\hline 6 & $\begin{array}{r}63 \\
122 \\
139 \\
105 \\
111\end{array}$ & $\begin{array}{r}97 \\
90 \\
83 \\
76 \\
125\end{array}$ & $\begin{array}{l}104 \\
117 \\
112 \\
125 \\
136\end{array}$ & $\begin{array}{l}66 \\
57 \\
54 \\
44 \\
41\end{array}$ & $\begin{array}{r}50 \\
128 \\
116 \\
131 \\
144\end{array}$ & $\begin{array}{l}148 \\
131 \\
105 \\
148 \\
140\end{array}$ & \begin{tabular}{r|}
144 \\
118 \\
105 \\
66 \\
33
\end{tabular} & \begin{tabular}{r|}
57 \\
111 \\
60 \\
80 \\
76
\end{tabular} & $\begin{array}{l}148 \\
148 \\
148 \\
152 \\
148\end{array}$ & $\begin{array}{r}36 \\
45 \\
115 \\
47 \\
115\end{array}$ & $\begin{array}{l}57 \\
58 \\
62 \\
36 \\
46\end{array}$ & $\frac{4}{2}$ \\
\hline 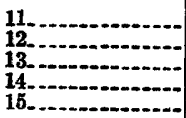 & $\begin{array}{r}115 \\
83 \\
90 \\
120 \\
107\end{array}$ & $\begin{array}{c}111 \\
111 \\
107 \\
90 \\
100\end{array}$ & $\begin{array}{r}144 \\
122 \\
97 \\
83 \\
73\end{array}$ & $\begin{array}{l}38 \\
38 \\
35 \\
50 \\
64\end{array}$ & $\begin{array}{l}152 \\
148 \\
148 \\
148 \\
144\end{array}$ & $\begin{array}{r}148 \\
144 \\
69 \\
133 \\
129\end{array}$ & $\begin{array}{r}60 \\
63 \\
63 \\
132 \\
111\end{array}$ & $\begin{array}{r}76 \\
152 \\
87 \\
111 \\
160\end{array}$ & $\begin{array}{l}148 \\
136 \\
125 \\
125 \\
140\end{array}$ & $\begin{array}{l}152 \\
152 \\
148 \\
135 \\
123\end{array}$ & $\begin{array}{r}97 \\
93 \\
134 \\
134 \\
91\end{array}$ & $\begin{array}{l}4 \\
4 \\
7\end{array}$ \\
\hline 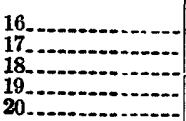 & $\begin{array}{r}122 \\
144 \\
133 \\
125 \\
97\end{array}$ & $\begin{array}{l}136 \\
144 \\
140 \\
114 \\
120\end{array}$ & $\begin{array}{r}76 \\
66 \\
64 \\
136 \\
83\end{array}$ & $\begin{array}{l}41 \\
38 \\
36 \\
38 \\
33\end{array}$ & $\begin{array}{l}125 \\
100 \\
133 \\
118 \\
109\end{array}$ & $\begin{array}{l}122 \\
133 \\
144 \\
129 \\
118\end{array}$ & $\begin{array}{r}83 \\
66 \\
76 \\
122 \\
114\end{array}$ & $\begin{array}{r}164 \\
0 \\
0 \\
0 \\
0\end{array}$ & $\begin{array}{r}118 \\
104 \\
97 \\
90 \\
83\end{array}$ & $\begin{array}{r}82 \\
79 \\
135 \\
90 \\
70\end{array}$ & $\begin{array}{l}60 \\
51 \\
45 \\
39 \\
36\end{array}$ & $\begin{array}{l}4 t \\
38\end{array}$ \\
\hline 西 & $\begin{array}{r}83 \\
119 \\
129 \\
140 \\
148\end{array}$ & $\begin{array}{l}111 \\
118 \\
122 \\
136 \\
104\end{array}$ & $\begin{array}{l}70 \\
60 \\
57 \\
54 \\
\mathbf{5 0}\end{array}$ & $\begin{array}{l}38 \\
54 \\
35 \\
33 \\
\mathbf{3 2}\end{array}$ & $\begin{array}{r}140 \\
107 \\
93 \\
90 \\
76\end{array}$ & $\begin{array}{r}114 \\
104 \\
97 \\
114 \\
133\end{array}$ & $\begin{array}{r}90 \\
152 \\
148 \\
132 \\
107\end{array}$ & $\begin{array}{r}0 \\
0 \\
0 \\
41 \\
118\end{array}$ & $\begin{array}{l}80 \\
73 \\
66 \\
63 \\
60\end{array}$ & $\begin{array}{l}63 \\
57 \\
51 \\
52 \\
45\end{array}$ & $\begin{array}{l}35 \\
36 \\
66 \\
62 \\
38\end{array}$ & 3 \\
\hline - & $\begin{array}{l}148 \\
148 \\
140 \\
125 \\
114 \\
110\end{array}$ & $\begin{array}{r}90 \\
84 \\
111 \\
76 \\
65 \\
60\end{array}$ & $\begin{array}{l}44 \\
\mathbf{4 4} \\
54 \\
97 \\
50\end{array}$ & $\begin{array}{r}30 \\
78 \\
110 \\
54 \\
64 \\
55\end{array}$ & $\begin{array}{l}70 \\
63 \\
60 \\
57 \\
60\end{array}$ & $\begin{array}{l}148 \\
129 \\
140 \\
144 \\
125 \\
107\end{array}$ & $\begin{array}{l}90 \\
76 \\
76 \\
66 \\
60 \\
57\end{array}$ & \begin{tabular}{r}
125 \\
111 \\
152 \\
\hdashline \\
\end{tabular} & $\begin{array}{l}57 \\
54 \\
50 \\
47 \\
44 \\
41\end{array}$ & $\begin{array}{c}42 \\
39 \\
38 \\
36 \\
35 \\
-\cdots\end{array}$ & $\begin{array}{l}34 \\
68 \\
61 \\
36 \\
31 \\
30\end{array}$ & $\begin{array}{l}27 \\
24 \\
23 \\
22 \\
24\end{array}$ \\
\hline \multirow{2}{*}{\multicolumn{3}{|c|}{ Mont }} & \multicolumn{5}{|c|}{ Million gallons a day } & \multirow{2}{*}{\multicolumn{2}{|c|}{$\begin{array}{l}\text { Second- } \\
\text { feet } \\
\text { (mean) }\end{array}$}} & \multicolumn{3}{|c|}{ Total run-off } \\
\hline & & & & Taximum & Min & mam & Mean & & & $\begin{array}{l}\text { Million } \\
\text { gallons }\end{array}$ & Acre & e-feet \\
\hline \multicolumn{4}{|c|}{ 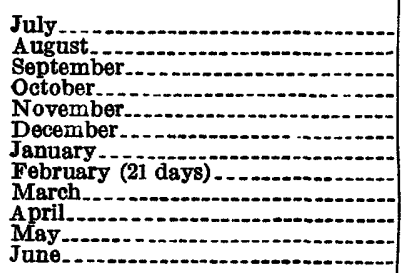 } & $\begin{array}{l}148 \\
144 \\
144 \\
110 \\
152 \\
148 \\
152 \\
164 \\
152 \\
152 \\
134 \\
113\end{array}$ & & $\begin{array}{r}63 \\
60 \\
44 \\
30 \\
37 \\
44 \\
33 \\
0 \\
41 \\
35 \\
28 \\
22\end{array}$ & $\begin{array}{c}117 \\
108 \\
90.5 \\
53.7 \\
98.4 \\
115 \\
96.8 \\
906.8 \\
106 \\
72.8 \\
54.4 \\
43.3\end{array}$ & \multicolumn{2}{|c|}{$\begin{array}{l}181 \\
167 \\
140 \\
83.1 \\
152 \\
178 \\
150 \\
142 \\
164 \\
113 \\
84.2 \\
67.0\end{array}$} & $\begin{array}{l}3,620 \\
3,340 \\
2,720 \\
1,660 \\
2,950 \\
3,580 \\
3,000 \\
1,930 \\
3,280 \\
2,190 \\
1,680 \\
1,300\end{array}$ & & $\begin{array}{r}11,100 \\
10,300 \\
8,330 \\
5,110 \\
9,060 \\
10,900 \\
9,220 \\
5,920 \\
10,100 \\
6,710 \\
5,180 \\
3,990\end{array}$ \\
\hline \multicolumn{4}{|l|}{ The year.- } & 164 & & 0 & 87.3 & 15 & 35 & 1,200 & & 95,90 \\
\hline
\end{tabular}




\section{HONOMANU STREAM NEAR KEANAE, MAUI}

Location.-Water-stage recorder 500 feet above Spreckels ditch intake and trail bridge and 3 miles by trail northwest of Upper Keanae.

Drainage AREA.-3.3 square miles.

ReCORDS AVAILABLE. - November, 1913, to June, 1929.

EXTREmes.- Maximum discharge during year, 1,220 million gallons a day or 1,890 second-feet Feb. 17 (gage height, 8.96 feet); minimum, 0.9 million gallons a day or 1.4 second-feet June 29 .

1913-1929: Maximum discharge, that of Feb. 17, 1929; minimum, 0.08 million gallons a day or 0.12 second-foot Mar. 24, 1928.

Remarks. - Records good except those for high stages, which are poor. Estimated record fair for low water. No diversions.

Discharge, in million gallons a day, 1928-29

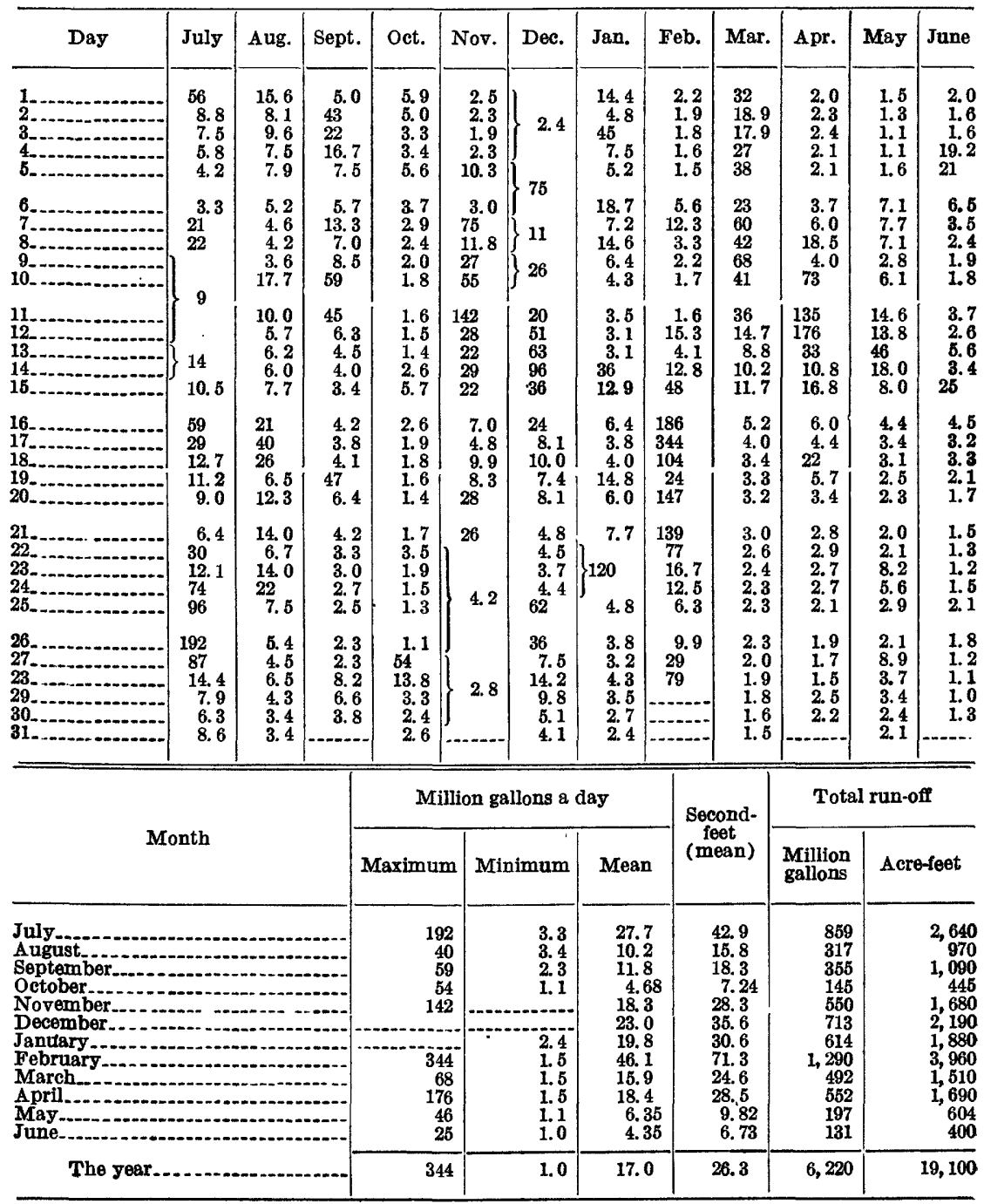




\section{HAIPUAETA STREAM TEAR HUELO, MAUI}

Location.-Water-stage recorder 200 feet above inflow of Spreckels ditch and $31 / 4$ miles southeast of Kailua.

Drainage area. - 1.1 square miles.

Records available.-October, 1913, to June, 1929.

EXTREMES.- Maximum discharge during year, 582 million gallons a day or 900 second-feet Feb. 17 (gage height, 6.25 feet); minimum, 1.4 million gallons a day or 2.2 second-feet Oct. $26,27$.

1913-1929: Maximum discharge, that of Feb. 17, 1929; minimum, 0.3 million gallons a day or 0.5 second-foot frequently during December, 1919.

REMARKS.- Records fair for ordinary stages except those estimated, which are poor; records for extremely high stages poor, No diversions. Intake plugged often during year.

Discharge, in million gallons a day, 1928-29

\begin{tabular}{|c|c|c|c|c|c|c|c|c|c|c|c|c|}
\hline Day & July & Ang. & Sept. & Oct. & Nov. & Dec. & Jan. & Feb. & Mar. & A pr. & May & June \\
\hline $\begin{array}{l}1 \\
2 \\
3 \\
4 \\
5\end{array}$ & $\begin{array}{l}43 \\
8.3 \\
6.9 \\
5.6 \\
4.7\end{array}$ & $\begin{array}{r}16.2 \\
8.6 \\
10.2 \\
8.9 \\
9.3\end{array}$ & $\begin{array}{l}5.2 \\
45 \\
23 \\
17.4 \\
8.8\end{array}$ & $\begin{array}{l}6.1 \\
5.8 \\
4.0 \\
4.3 \\
7.4\end{array}$ & \begin{tabular}{r|}
3.4 \\
3.1 \\
2.7 \\
3.1 \\
16.7
\end{tabular} & $\begin{array}{r}-2.8 \\
2.7 \\
3.2 \\
4.0 \\
64\end{array}$ & $\begin{array}{r}16.2 \\
6.6 \\
34 \\
8.6 \\
6.8\end{array}$ & $\begin{aligned} & 2.0 \\
\text { a } & 1.6 \\
\text { a } & 1.6 \\
\text { - } & 1.4 \\
& 1.4\end{aligned}$ & $\begin{array}{l}24 \\
14.6 \\
15.4 \\
24 \\
33\end{array}$ & $\begin{array}{l}2.0 \\
2.3 \\
2.7 \\
2.4 \\
2.1\end{array}$ & $\begin{array}{r}2.0 \\
1.6 \\
1.5 \\
\times 1.5 \\
2.3\end{array}$ & $\begin{array}{r}2.7 \\
2.2 \\
2.1 \\
11.0 \\
13.0\end{array}$ \\
\hline $\begin{array}{r}6 \\
7 \\
8 \\
9 \\
9\end{array}$ & $\begin{array}{r}4.0 \\
14.4 \\
18.5 \\
7.6 \\
9.2\end{array}$ & $\begin{array}{r}5.9 \\
5.4 \\
4.5 \\
3.9 \\
11.0\end{array}$ & $\begin{array}{r}6.7 \\
12.2 \\
7.5 \\
11.5 \\
63\end{array}$ & $\begin{array}{r}\text { 5. } 0 \\
4.0 \\
3.8 \\
3.4 \\
\text { s. } 3.0\end{array}$ & $\begin{array}{l}3.9 \\
72 \\
13.5 \\
24 \\
37\end{array}$ & $\begin{array}{l}58 \\
16.1 \\
8.0 \\
20\end{array}$ & \begin{tabular}{l|}
20 \\
8.5 \\
22 \\
09.2 \\
-5.4
\end{tabular} & $\begin{array}{l}4.5 \\
8.9 \\
2.6 \\
1.7 \\
1.5\end{array}$ & $\begin{array}{l}17.6 \\
38 \\
28 \\
59 \\
27\end{array}$ & $\begin{array}{r}3.2 \\
5.0 \\
14.0 \\
4.7 \\
54\end{array}$ & $\begin{array}{l}7.4 \\
8.7 \\
8.3 \\
3.1 \\
4.5\end{array}$ & $\begin{array}{r}6.0 \\
-4.1 \\
-2.7 \\
2.3 \\
2.3\end{array}$ \\
\hline 112 & $\begin{array}{r}8.7 \\
6.9 \\
9.1 \\
13.4 \\
7.2\end{array}$ & $\begin{array}{l}9.8 \\
7.0 \\
7.1 \\
6.5 \\
7.8\end{array}$ & $\begin{array}{l}25 \\
8.2 \\
6.2 \\
5.2 \\
4.8\end{array}$ & $\begin{array}{r}2.6 \\
2.5 \\
2.4 \\
4.0 \\
7.1\end{array}$ & $\begin{array}{l}90 \\
19.4 \\
21 \\
35 \\
23\end{array}$ & $\begin{array}{l}44 \\
55 \\
81 \\
33\end{array}$ & $\begin{array}{c}4.5 \\
3.9 \\
4.0 \\
32 \\
12.5\end{array}$ & $\begin{array}{r}1.5 \\
11.6 \\
3.2 \\
11.5 \\
42\end{array}$ & $\begin{array}{r}27 \\
10.2 \\
6.9 \\
9.0 \\
14.3\end{array}$ & $\begin{aligned} 77 \\
96 \\
17.7 \\
8.2 \\
10.5\end{aligned}$ & $\begin{array}{r}11.8 \\
9.2 \\
29 \\
15.2 \\
7.6\end{array}$ & $\begin{array}{r}3.9 \\
3.1 \\
5.1 \\
3.6 \\
18.8\end{array}$ \\
\hline $\begin{array}{l}16 \\
17 \\
18\end{array}$ & \begin{tabular}{l|}
45 \\
24 \\
10.4 \\
10.0 \\
7.2
\end{tabular} & \begin{tabular}{l|}
28 \\
56 \\
19.8 \\
7.4 \\
15.0
\end{tabular} & $\begin{array}{r}5.4 \\
4.9 \\
5.2 \\
54 \\
8.0\end{array}$ & $\begin{array}{l}3.5 \\
2.8 \\
2.6 \\
2.5 \\
2.3\end{array}$ & $\begin{array}{r}9.2 \\
6.8 \\
11.5 \\
9.2 \\
25\end{array}$ & $\begin{array}{r}30 \\
9.8 \\
12.9 \\
8.2 \\
8.7\end{array}$ & $\begin{array}{r}6.9 \\
4.5 \\
5.4 \\
11.8 \\
5.9\end{array}$ & $\begin{array}{r}110 \\
156 \\
52 \\
26 \\
66\end{array}$ & $\begin{array}{r}6.0 \\
-4.7 \\
-4.2 \\
-4.0 \\
-4.0\end{array}$ & $\begin{array}{c}5.4 \\
4.9 \\
22 \\
5.9 \\
4.2\end{array}$ & $\begin{array}{r}4.7 \\
3.7 \\
\text { a } 3.4 \\
-3.0 \\
2.7\end{array}$ & $\begin{array}{l}5.2 \\
3.7 \\
3.8 \\
2.9 \\
2.6\end{array}$ \\
\hline $\begin{array}{l}21 \\
22 \\
24\end{array}$ & $\begin{array}{c}5.1 \\
24 \\
9.1 \\
57 \\
75\end{array}$ & $\begin{array}{r}10.4 \\
7.7 \\
13.9 \\
17.7 \\
6.7\end{array}$ & $\begin{array}{r}5.3 \\
4.4 \\
3.9 \\
3.7 \\
\text { a. } 3\end{array}$ & $\begin{aligned} & 2.7 \\
& 5.7 \\
&-5.4 \\
&-3.4 \\
& \therefore 2.3\end{aligned}$ & $\begin{array}{r}18.5 \\
6.7 \\
5.4 \\
5.2 \\
5.8\end{array}$ & $\begin{array}{r}6.5 \\
5.8 \\
4.9 \\
6.8 \\
61\end{array}$ & $\begin{array}{c}8.7 \\
154 \\
24 \\
5.2 \\
3.4\end{array}$ & $\begin{array}{l}60 \\
48 \\
11.1 \\
12.7 \\
5.6\end{array}$ & $\begin{array}{r}-3.6 \\
2.8 \\
-2.5 \\
-2.3 \\
2.3\end{array}$ & $\begin{array}{l}3.4 \\
3.3 \\
3.1 \\
3.2 \\
\text { 2. } 6\end{array}$ & $\begin{array}{r}2.7 \\
\times 2.9 \\
6.7 \\
5.0 \\
2.9\end{array}$ & $\begin{array}{r}2.6 \\
-2.6 \\
1.8 \\
2.1 \\
2.6\end{array}$ \\
\hline 28 & $\begin{array}{r}134 \\
44 \\
12.1 \\
8.0 \\
6.7 \\
8.5\end{array}$ & $\begin{array}{l}5.2 \\
5.4 \\
7.3 \\
4.8 \\
4.2 \\
4.0\end{array}$ & $\begin{array}{r}-3.2 \\
3.1 \\
7.6 \\
12.8 \\
4.3\end{array}$ & \begin{tabular}{r|r}
2.0 & \\
51 & \\
13.9 & \\
4.2 & \\
3.2 & \\
3.0 & --
\end{tabular} & $\begin{array}{r}5.4 \\
4.0 \\
\text { a } 3.6 \\
\text { a } 3.3 \\
\text { a } 3.4 \\
\end{array}$ & \begin{aligned} \multicolumn{1}{c}{26} \\
8.4 \\
14.8 \\
11.8 \\
6.0 \\
5.0\end{aligned} & $\begin{array}{r}2.8 \\
2.5 \\
3.9 \\
02.9 \\
22.1 \\
-2.0\end{array}$ & $\begin{array}{c}6.8 \\
15.1 \\
48\end{array}$ & $\begin{array}{l}2.2 \\
2.0 \\
1.9 \\
1.7 \\
1.7 \\
1.6\end{array}$ & $\begin{array}{r}2.4 \\
-2.6 \\
2.0 \\
\times 2.5 \\
-2.7 \\
\end{array}$ & $\begin{array}{l}2.4 \\
4.7 \\
3.5 \\
4.1 \\
3.1 \\
2.9\end{array}$ & $\begin{array}{r}2.3 \\
01.8 \\
1.6 \\
1.6 \\
1.9 \\
\\
-.-\end{array}$ \\
\hline \multirow{2}{*}{\multicolumn{4}{|c|}{ Month }} & \multicolumn{4}{|c|}{ Million gallons a day } & \multirow{2}{*}{\multicolumn{2}{|c|}{$\begin{array}{c}\text { Second- } \\
\text { feet } \\
\text { (mean) }\end{array}$}} & \multicolumn{3}{|c|}{ Total run-off } \\
\hline & & & & Maximum & Min & mum & Mean & & & $\begin{array}{l}\text { Million } \\
\text { gallons }\end{array}$ & Acr & feet \\
\hline $\begin{array}{l}\text { July } \\
\text { August. } \\
\text { September } \\
\text { October } \\
\text { November } \\
\text { December. } \\
\text { January } \\
\text { February } \\
\text { March } \\
\text { April } \\
\text { May. } \\
\text { June }\end{array}$ & $\because$ & - & & $\begin{array}{r}134 \\
56 \\
63 \\
51 \\
90 \\
81 \\
154 \\
156 \\
59 \\
96 \\
29 \\
18.8\end{array}$ & & $\begin{array}{l}4.0 \\
3.9 \\
3.1 \\
2.0 \\
2.7 \\
2.7 \\
2.0 \\
1.4 \\
1.6 \\
2.0 \\
1.5 \\
1.6\end{array}$ & $\begin{array}{r}20.9 \\
10.8 \\
12.6 \\
5.6 \\
16.4 \\
21.1 \\
14.2 \\
25.5 \\
12.8 \\
12.4 \\
5.5 \\
4.0\end{array}$ & & $\begin{array}{l}32.3 \\
6.7 \\
9.5 \\
8.77 \\
25.4 \\
32.6 \\
22.0 \\
39.5 \\
9.8 \\
19.2 \\
8.59 \\
6.30\end{array}$ & $\begin{array}{l}648 \\
336 \\
379 \\
176 \\
491 \\
653 \\
440 \\
714 \\
396 \\
372 \\
172 \\
122\end{array}$ & & $\begin{array}{r}1,990 \\
1,030 \\
1,160 \\
539 \\
1,510 \\
2,010 \\
1,350 \\
2,190 \\
1,220 \\
1,140 \\
528 \\
375\end{array}$ \\
\hline The year & & & & 156 & & 1.4 & 13.4 & & 20.7 & 4,900 & & 15,000 \\
\hline
\end{tabular}




\section{SPRECKELS DITCH AT HAIPUAENA WEIR, NEAR HUELO, MAOI}

Location.-Water-stage recorder between Haipuaena and Puohokamoa Streams on Spreckels ditch trail, 31/4 miles southeast of Kailua.

Records aVAILABLe. - April, 1922, to June, 1929.

EXTrEmes.-Maximum discharge during year, 81 million gallons a day or 125 second-feet Feb. 8 and Apr. 26 (gage height, 2.83 feet); minimum, 0.8 million gallons a day or 1.2 second-feet June 29 .

1922-1929: Maximum discharge, that of Feb. 8 and Apr. 26, 1929; no flow nearly entire time Apr. 9-23, 1925, when water was shut out of ditch.

REMARKS.-Records excellent except those estimated, which are poor. Regulated by gates and spillways. Since May, 1928, East Maui Irrigation Co.'s power plant has diverted continuously about 4 million gallons a day just above station. Spreckels ditch diverts from all streams between Nuaailua and Kailua above Koolau ditch east of Puohokamoa and below Koolau ditch west of Puohokamoa.

Discharge, in million gallons a day, 1928-29

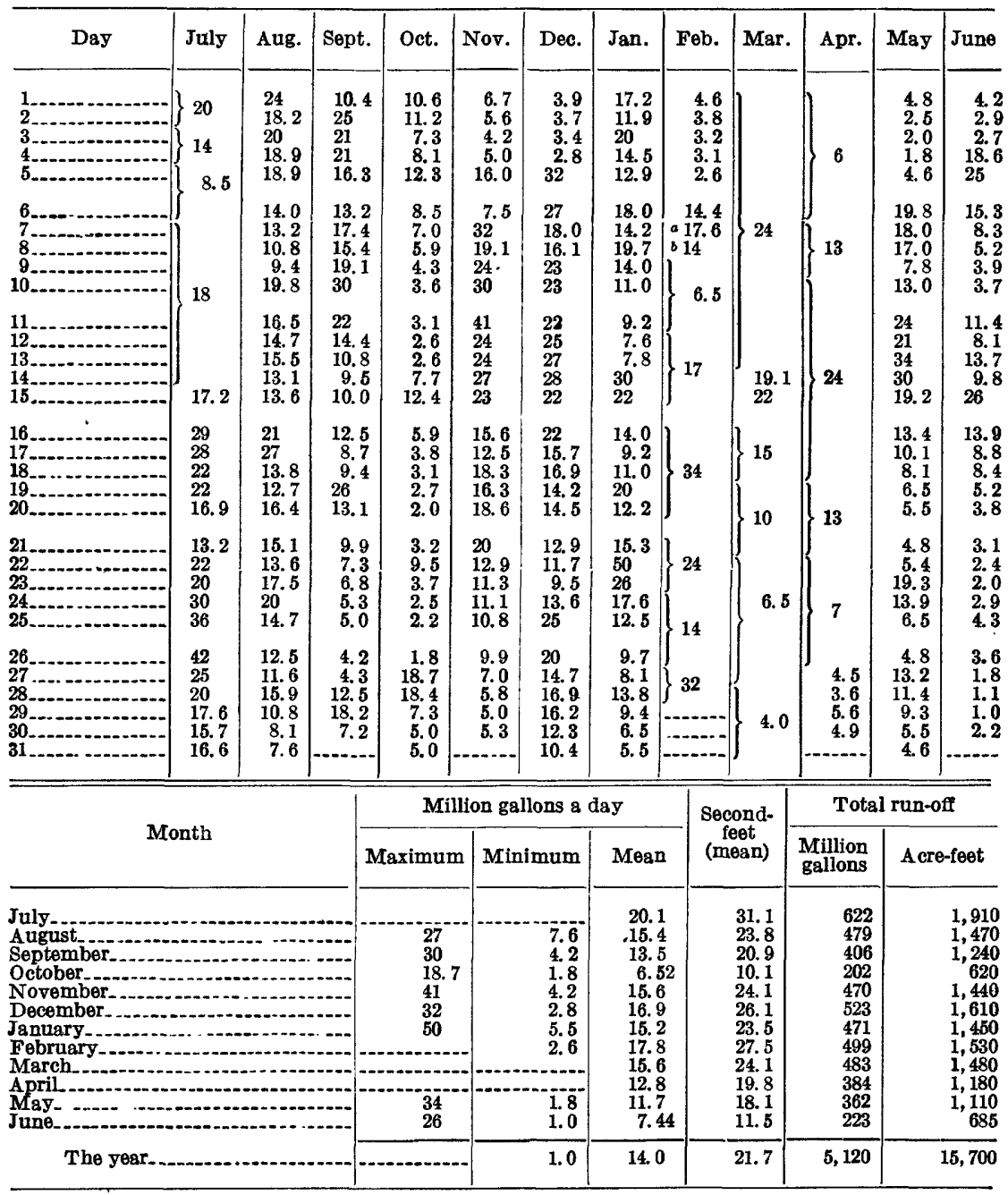

a Partly estimated.

- Estimated. 


\section{PUOHOKAMOA STREAM NEAR HUELO, MAUI}

Location.-Water-stage recorder just above Spreckels ditch inflow and trai. crossing and 3 miles southeast of Kailua.

DRAINAGE AREA.-2.6 square miles.

Records AVAILABLE.-December, 1910, to June, 1929.

ExTremes.-Maximum discharge during year, 920 million gallons a day or 1,420 second-feet Feb. 17 (gage height, 7.20 feet); minimum, 1.0 millon gallons a day or 1.6 second-feet Oct. 27.

1910-1929: Maximum discharge, 1,100 million gallons a day or 1,700 second-feet Jan. 14, 1923 (gage height, 7.85 feet); minimum, 0.4 million gallons a day or 0.6 second-foot Oct. 26, 1917.

REMARKs.- Records fair except those for high stages and estimates, which are poor. Kula pipe line diverts small amounts of water above station at elevation 4,300 feet.

Discharge, in million gallons a day, 1928-29

\begin{tabular}{|c|c|c|c|c|c|c|c|c|c|c|c|c|}
\hline Day & July & Aug. & Sept. & Oct. & Nov.l & Dec. & Jan. & Feb. & Mar. & Apr. & May & June \\
\hline & \begin{tabular}{|c}
62 \\
15.4 \\
$a 13.3$ \\
$\therefore 11.4$ \\
8.6
\end{tabular} & $\begin{array}{l}28 \\
15.4 \\
a 16.6 \\
015 \\
17.6\end{array}$ & $\begin{array}{l}7.8 \\
71 \\
38 \\
30 \\
15.4\end{array}$ & $\begin{array}{r}8.4 \\
7.8 \\
5.2 \\
5.8 \\
11.4\end{array}$ & $\begin{array}{r}4.8 \\
4.2 \\
3.5 \\
3.5 \\
17.1\end{array}$ & $\begin{array}{r}63.9 \\
3.7 \\
3.4 \\
3.2 \\
111\end{array}$ & $\begin{array}{l}21 \\
14.9 \\
49 \\
18.0 \\
14.3\end{array}$ & $\begin{array}{l}7.1 \\
6.5 \\
6.1 \\
5.7 \\
5.1\end{array}$ & $\begin{array}{l}61 \\
41 \\
41 \\
67 \\
84\end{array}$ & $\begin{array}{l}4.7 \\
\text { 5. } 2 \\
6.1 \\
5.1 \\
4.5\end{array}$ & $\begin{array}{l}4.7 \\
4.0 \\
3.7 \\
3.5 \\
4.1\end{array}$ & $\begin{array}{r}3.9 \\
3.5 \\
3.4 \\
15.3 \\
22\end{array}$ \\
\hline $\begin{array}{r}6 \ldots \\
7 \ldots \\
8 \ldots \\
9 \ldots \\
10 \ldots\end{array}$ & $\begin{array}{r}6.7 \\
419.1 \\
32 \\
13.3 \\
16.6\end{array}$ & $\begin{array}{c}a 11.4 \\
611 \\
a 9.7 \\
8.5 \\
a 24\end{array}$ & $\begin{array}{c}11.4 \\
19.7 \\
12.8 \\
18.8 \\
106\end{array}$ & $\begin{array}{l}6.8 \\
5.1 \\
4.4 \\
3.7 \\
3.4\end{array}$ & \begin{tabular}{|c|}
5.1 \\
113 \\
21 \\
32 \\
49
\end{tabular} & $\begin{array}{l}64 \\
20 \\
20 \\
43 \\
45\end{array}$ & $\begin{array}{l}38 \\
17.4 \\
39 \\
16.6 \\
12.3\end{array}$ & $\begin{array}{l}28 \\
25 \\
8.9 \\
6.4 \\
5.4\end{array}$ & $\begin{array}{r}40 \\
83 \\
65 \\
144 \\
62\end{array}$ & $\begin{array}{c}7.3 \\
10.5 \\
28 \\
7.3 \\
107^{3}\end{array}$ & $\begin{array}{r}15.7 \\
16.0 \\
12.6 \\
6.0 \\
\cdot 8.5\end{array}$ & 3 \\
\hline $\begin{array}{l}12 \ldots \ldots \\
13 \\
15 \\
15\end{array}$ & $\begin{array}{r}15.0 \\
a 10.5 \\
13.3 \\
a 19.9 \\
a 12.3\end{array}$ & $\begin{array}{rr}a & 17.0 \\
a & 13.0 \\
b & 14 \\
a & 15.4 \\
14.1\end{array}$ & $\begin{array}{l}42 \\
14.3 \\
11.4 \\
8.9 \\
8.5\end{array}$ & $\begin{array}{l}3.0 \\
2.7 \\
2.5 \\
5.5 \\
8.7\end{array}$ & $\begin{array}{r}121 \\
27 \\
34 \\
60 \\
33\end{array}$ & $\begin{array}{r}33 \\
71 \\
88 \\
142 \\
41\end{array}$ & $\begin{array}{c}11.4 \\
9.7 \\
10.1 \\
53 \\
23\end{array}$ & $\begin{array}{l}5.8 \\
34 \\
10.7 \\
27 \\
95\end{array}$ & $\begin{array}{l}65 \\
25 \\
18.4 \\
31 \\
38\end{array}$ & $\begin{array}{r}168 \\
212 \\
39 \\
21 \\
22\end{array}$ & $\begin{array}{l}28 \\
19.8 \\
61 \\
38 \\
17.8\end{array}$ & $\begin{array}{r}6 . \\
5 . \\
9 . \\
5 . \\
\mathbf{3 9}\end{array}$ \\
\hline $\begin{array}{l}16 \ldots \ldots \\
17 \\
18 \\
19\end{array}$ & $\begin{array}{c}77 \\
40 \\
\times 19.5 \\
19.5 \\
14.3\end{array}$ & $\begin{array}{l}045 \\
a 92 \\
a 58 \\
615 \\
624\end{array}$ & $\begin{array}{r}8.9 \\
7.8 \\
8.2 \\
96 \\
13.8\end{array}$ & $\begin{array}{l}4.2 \\
3.2 \\
2.9 \\
2.7 \\
2.3\end{array}$ & $\begin{array}{l}15.4 \\
11.4 \\
17.8 \\
14.4 \\
34\end{array}$ & $\begin{array}{l}47 \\
19.5 \\
26 \\
18.0 \\
16.6\end{array}$ & $\begin{array}{l}14.3 \\
10.5 \\
11.0 \\
17.7 \\
11.4\end{array}$ & $\begin{array}{r}207 \\
281 \\
115 \\
65 \\
140\end{array}$ & $\begin{array}{r}16.6 \\
13.3 \\
11.4 \\
10.5 \\
9.7\end{array}$ & \begin{tabular}{r|}
13.3 \\
12.3 \\
54 \\
13.3 \\
9.7
\end{tabular} & $\begin{array}{r}10.5 \\
8.3 \\
6.9 \\
5.9 \\
5.2\end{array}$ & 4 \\
\hline $\begin{array}{l}21 \ldots . . . \\
22 \\
23 \\
24 \\
25\end{array}$ & $\begin{array}{l}10.5 \\
043 \\
16.6 \\
b 100 \\
129\end{array}$ & $\begin{array}{ll}b & 17 \\
b & 14 \\
b & 20 \\
b & 30 \\
b & 14\end{array}$ & $\begin{array}{l}9.7 \\
7.4 \\
6.3 \\
5.6 \\
5.1\end{array}$ & $\begin{array}{l}2.6 \\
5.4 \\
2.9 \\
2.3 \\
2.1\end{array}$ & $\begin{array}{r}27 \\
11.4 \\
8.9 \\
a 8.3 \\
212.6\end{array}$ & $\begin{array}{r}14.3 \\
13: 3 \\
11.4 \\
14.8 \\
108\end{array}$ & $\begin{array}{c}15.2 \\
249 \\
96 \\
18.0 \\
14.3\end{array}$ & $\begin{array}{c}102 \\
117 \\
30 \\
62 \\
16.6\end{array}$ & $\begin{array}{l}8.9 \\
7.8 \\
7.0 \\
6.5 \\
6.1\end{array}$ & $\begin{array}{l}8.3 \\
7.6 \\
7.0 \\
7.3 \\
\text { 5. }\end{array}$ & $\begin{array}{r}4.9 \\
4.9 \\
13.4 \\
10.4 \\
5.4\end{array}$ & $\begin{array}{l}3 \\
3 \\
4\end{array}$ \\
\hline $\begin{array}{l}26 \ldots \\
27 \\
28 \\
29 \\
30 \\
31\end{array} \ldots$ & $\begin{array}{l}222 \\
: 73 \\
b 27 \\
\cdot 18.0 \\
15.4 \\
15.0\end{array}$ & $\begin{array}{l}010 \\
110 \\
013.3 \\
a 7.9 \\
a 6.9 \\
a 6.5\end{array}$ & $\begin{array}{r}4.5 \\
4.4 \\
9.2 \\
017.3 \\
6.2 \\
\hdashline-\cdots\end{array}$ & $\begin{array}{l}1.6 \\
85 \\
20 \\
6.4 \\
4.7 \\
4.2\end{array}$ & $\begin{array}{r}12.2 \\
6.3 \\
5.3 \\
4.7 \\
4.5\end{array}$ & $\begin{array}{l}38 \\
16.6 \\
28 \\
22 \\
13.3 \\
12.3\end{array}$ & $\begin{array}{r}12.3 \\
11.0 \\
15.2 \\
11.4 \\
8.9 \\
7.8\end{array}$ & 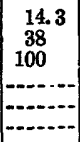 & $\begin{array}{l}5.6 \\
5.2 \\
4.9 \\
4.6 \\
4.4 \\
4.2\end{array}$ & $\begin{array}{l}5.4 \\
5.1 \\
4.7 \\
5.2 \\
4.9\end{array}$ & $\begin{array}{l}4.6 \\
8.3 \\
7.4 \\
7.2 \\
4.5 \\
4.2\end{array}$ & 2. \\
\hline
\end{tabular}

\begin{tabular}{|c|c|c|c|c|c|c|}
\hline \multirow{2}{*}{$\cdot$} & \multicolumn{3}{|c|}{ Million gallons a day } & \multirow{2}{*}{$\begin{array}{c}\text { Second- } \\
\text { feet } \\
\text { (mean) }\end{array}$} & \multicolumn{2}{|c|}{ Total run-off } \\
\hline & Maximum & Minimum & Mean & & $\begin{array}{l}\text { Million } \\
\text { gallons }\end{array}$ & Acre-feet \\
\hline $\begin{array}{l}\text { July. } \\
\text { August } \\
\text { September } \\
\text { October } \\
\text { November } \\
\text { December } \\
\text { January } \\
\text { February } \\
\text { March } \\
\text { April } \\
\text { May } \\
\text { June }\end{array}$ & $\begin{array}{r}222 \\
92 \\
106 \\
85 \\
121 \\
142 \\
249 \\
281 \\
144 \\
212 \\
61 \\
39\end{array}$ & $\begin{array}{l}\text { 6. } 7 \\
6.5 \\
4.4 \\
1.6 \\
3.5 \\
3.2 \\
7.8 \\
5.1 \\
4.2 \\
4.5 \\
3.5 \\
2.4\end{array}$ & $\begin{array}{l}35.8 \\
19.8 \\
20.9 \\
7.64 \\
24.1 \\
35.8 \\
28.1 \\
55.9 \\
32.0 \\
27.0 \\
11.5 \\
6.80\end{array}$ & $\begin{array}{l}55.4 \\
30.6 \\
32.3 \\
11.8 \\
37.3 \\
55.4 \\
43.5 \\
86.5 \\
49.5 \\
41.8 \\
17.8 \\
10.5\end{array}$ & $\begin{array}{r}1,110 \\
614 \\
626 \\
237 \\
722 \\
1,110 \\
872 \\
1.560 \\
992 \\
812 \\
355 \\
204\end{array}$ & $\begin{array}{r}3,410 \\
1,880 \\
1,920 \\
727 \\
2,220 \\
3,410 \\
2,670 \\
4,800 \\
3,040 \\
2,490 \\
1,090 \\
626\end{array}$ \\
\hline The year... & 281 & 1.6 & 25.3 & 39.1 & 0,210 & 28,300 \\
\hline
\end{tabular}




\section{PUOHOKAMOA INTAKE OF KOOLAU DITCH NEAR HUELO, MAUI}

Locatron. - Water-stage recorder 20 feet below intake on short feeder ditch from Puohokamoa Stream to Koolau ditch and 3 miles southeast of Kailua.

Records available.-March, 1922, to June, 1929.

EXTREMES.- Maximum discharge during year, 33 million gallons a day or 51 second-feet Apr. 10 (gage height, 1.80 feet); minimum, 0.1 million gallons a day or 0.2 second-foot June 29 .

1922-1929: Maximum discharge, 88 million gallons a day or 136 secondfeet Oct. 22, 1922 (gage height, 3.04 feet); a higher discharge may have occurred during period of no record Sept. 4-15, 1923 . Minimum discharge, that of June $29,1929$.

REMARKs. - Records excellent for ordinary stages, good for high stages, and fair for estimated periods. Diverts water from Puohokamoa Stream into Koolau ditch. Regulated by gates.

Discharge, in million gallons a day, 1928-29

\begin{tabular}{|c|c|c|c|c|c|c|c|c|c|c|c|c|}
\hline Day & July & Aug. & Sept. & Oct. & Nov. & Dec. & Jan. & Feb. & Mar. & Apr. & May & June \\
\hline $\begin{array}{l}1 \\
2 \\
3 \\
4 \\
5\end{array}$ & $\begin{array}{l}13.0 \\
11.7 \\
11.4 \\
11.2 \\
11.0\end{array}$ & $\begin{array}{l}15.4 \\
15.0 \\
15.4 \\
15.0 \\
15.2\end{array}$ & $\begin{array}{l}14.1 \\
16.6 \\
15.4 \\
15.4 \\
14.7\end{array}$ & $\begin{array}{l}14.3 \\
14.3 \\
13.6 \\
13.9 \\
14.7\end{array}$ & $\begin{array}{r}9.4 \\
9.4 \\
9.0 \\
8.8 \\
10.3\end{array}$ & $\begin{array}{l}9.0 \\
8.8 \\
8.8 \\
8.7 \\
9.2\end{array}$ & $\begin{array}{l}17.1 \\
23 \\
26 \\
23 \\
20\end{array}$ & $\begin{array}{r}12.2 \\
9.7 \\
9.6 \\
9.6 \\
9.4\end{array}$ & $\begin{array}{l}22 \\
20 \\
20 \\
22 \\
22\end{array}$ & $\begin{array}{r}8.7 \\
9.2 \\
11.0 \\
12.6 \\
9.9\end{array}$ & 11 & 10 \\
\hline $\begin{array}{l}6 \\
7 \\
9 \\
9\end{array}$ & $\begin{array}{l}11.0 \\
11.9 \\
12.1 \\
11.2 \\
11.4\end{array}$ & $\begin{array}{l}14.7 \\
14.7 \\
14.3 \\
14.1 \\
15.4\end{array}$ & $\begin{array}{l}14.5 \\
15.0 \\
14.5 \\
15.0 \\
17.0\end{array}$ & $\begin{array}{l}14.1 \\
13.6 \\
13.4 \\
12.5 \\
12.1\end{array}$ & $\begin{array}{r}9.4 \\
11.6 \\
10.3 \\
10.6 \\
\mathbf{1 0 . 8}\end{array}$ & $\begin{array}{r}4.1 \\
3.7 \\
11.3 \\
15.4 \\
15.4\end{array}$ & $\begin{array}{l}23 \\
22 \\
23 \\
22 \\
13.2\end{array}$ & $\begin{array}{r}13.4 \\
16.6 \\
15.0 \\
12.3 \\
9.9\end{array}$ & $\begin{array}{l}20 \\
22 \\
22 \\
24 \\
22\end{array}$ & $\begin{array}{r}9.7 \\
9.6 \\
15.4 \\
17.8 \\
19.1\end{array}$ & 15 & 15 \\
\hline $\begin{array}{l}11 \ldots \\
12 \\
13 \\
14 \\
15\end{array}$ & $\begin{array}{l}11.4 \\
11.0 \\
11.2 \\
11.7 \\
11.2\end{array}$ & $\begin{array}{l}15.2 \\
15.0 \\
15.0 \\
14.7 \\
15.0\end{array}$ & $\begin{array}{l}15.4 \\
14.7 \\
14.3 \\
13.9 \\
13.9\end{array}$ & $\begin{array}{r}10.0 \\
9.2 \\
9.0 \\
9.7 \\
10.6\end{array}$ & $\begin{array}{l}12.1 \\
10.5 \\
10.6 \\
11.0 \\
10.6\end{array}$ & $\begin{array}{r}15.2 \\
16.6 \\
7.5 \\
.3 \\
.2\end{array}$ & $\begin{array}{l}14.7 \\
14.5 \\
14.5 \\
16.6 \\
16.6\end{array}$ & $\begin{array}{r}9.9 \\
17.8 \\
17.8 \\
18.9 \\
22\end{array}$ & $\begin{array}{l}22 \\
22 \\
22 \\
23 \\
\mathbf{2 3}\end{array}$ & $\begin{array}{l}11.9 \\
10.8 \\
18.1 \\
24 \\
24\end{array}$ & 19 & 16 \\
\hline $\begin{array}{l}16 \\
17 \\
18 \\
19 \\
20\end{array}$ & $\begin{array}{l}12.5 \\
12.3 \\
11.7 \\
11.7 \\
11.2\end{array}$ & $\begin{array}{l}16.6 \\
17.8 \\
16.6 \\
15.0 \\
15.4\end{array}$ & $\begin{array}{l}14.1 \\
13.9 \\
14.1 \\
16.6 \\
14.7\end{array}$ & $\begin{array}{r}10.1 \\
9.6 \\
9.6 \\
9.4 \\
9.2\end{array}$ & $\begin{array}{r}10.1 \\
9.7 \\
10.1 \\
10.1 \\
10.3\end{array}$ & $\begin{array}{r}.2 \\
.2 \\
.2 \\
9.8 \\
12.3\end{array}$ & $\begin{array}{l}15.2 \\
14.7 \\
14.7 \\
15.4 \\
14.7\end{array}$ & $\begin{array}{r}17.1 \\
.6 \\
.4 \\
.3 \\
.3\end{array}$ & $\begin{array}{l}22 \\
20 \\
20 \\
18.9 \\
17.8\end{array}$ & $\begin{array}{l}19.3 \\
14.1 \\
15.4 \\
13.9\end{array}$ & & $\begin{array}{l}15.4 \\
15.0 \\
14.7 \\
13.6 \\
10.2\end{array}$ \\
\hline $\begin{array}{l}21 \\
22 \\
24 \\
25\end{array}$ & $\begin{array}{l}11.0 \\
12.1 \\
11.7 \\
13.2 \\
13.9\end{array}$ & $\begin{array}{l}15.2 \\
15.0 \\
15.4 \\
15.4 \\
15.0\end{array}$ & $\begin{array}{l}14.3 \\
13.9 \\
13.6 \\
13.4 \\
13.2\end{array}$ & $\begin{array}{r}9.6 \\
10.3 \\
9.7 \\
9.2 \\
8.8\end{array}$ & $\begin{array}{r}10.5 \\
9.7 \\
9.7 \\
9.6 \\
9.7\end{array}$ & $\begin{array}{l}12.1 \\
11.9 \\
11.7 \\
12.1 \\
13.3\end{array}$ & $\begin{array}{l}15.0 \\
20 \\
16.6 \\
15.4 \\
14.7\end{array}$ & $\begin{array}{r}.3 \\
.3 \\
.3 \\
9.8 \\
20\end{array}$ & $\begin{array}{l}16.6 \\
15.4 \\
15.0 \\
14.5 \\
13.6\end{array}$ & 13 & 13 & $\begin{array}{l}5.2 \\
5.2 \\
5.2 \\
5.2 \\
5.4\end{array}$ \\
\hline $\begin{array}{l}26 \\
28 \\
28 \\
30 \\
31\end{array}$ & $\begin{array}{l}14.7 \\
15.4 \\
15.4 \\
15.0 \\
14.7 \\
14.7\end{array}$ & $\begin{array}{l}14.7 \\
14.5 \\
14.9 \\
14.1 \\
13.9 \\
13.6\end{array}$ & $\begin{array}{l}12.8 \\
12.3 \\
14.5 \\
15.2 \\
13.8 \\
15\end{array}$ & $\begin{array}{r}8.3 \\
10.3 \\
10.5 \\
9.6 \\
9.4 \\
9.2\end{array}$ & $\begin{array}{l}9.6 \\
9.4 \\
9.2 \\
9.2 \\
9.2 \\
9 .\end{array}$ & $\begin{array}{l}13.4 \\
12.1 \\
12.5 \\
12.3 \\
11.9 \\
11.7\end{array}$ & $\begin{array}{l}14.5 \\
14.3 \\
15.2 \\
14.5 \\
13.9 \\
13.6\end{array}$ & \begin{tabular}{l}
17.8 \\
15.4 \\
20 \\
\hdashline \\
\hdashline
\end{tabular} & $\begin{array}{r}11.7 \\
11.4 \\
11.0 \\
9.9 \\
8.7 \\
8.7\end{array}$ & {$\left[\begin{array}{c}11.9 \\
12.3 \\
a 12\end{array}\right.$} & 12 & $\begin{array}{r}\mathbf{5 . 2} \\
\mathbf{5 . 1} \\
\mathbf{5 . 1} \\
\mathbf{3 .} 0 \\
\mathbf{5 . 1} \\
\end{array}$ \\
\hline \multirow{2}{*}{\multicolumn{4}{|c|}{ Month }} & \multicolumn{4}{|c|}{ Million gallons a day } & \multirow{2}{*}{\multicolumn{2}{|c|}{$\begin{array}{l}\text { Second- } \\
\text { feet } \\
\text { (mean) }\end{array}$}} & \multicolumn{3}{|c|}{ Total run-off } \\
\hline & & & & Maximum & Min & limum & Mean & & & $\begin{array}{l}\text { Million } \\
\text { gallons }\end{array}$ & Acre & e-feet \\
\hline $\begin{array}{l}\text { July } \\
\text { August } \\
\text { September.. } \\
\text { October } \\
\text { November... } \\
\text { December... } \\
\text { January } \\
\text { February } \\
\text { March } \\
\text { April } \\
\text { May } \\
\text { June. }\end{array}$ & $\cdots$ & $\cdots$ & \begin{tabular}{c}
-- \\
\hdashline- \\
\hdashline- \\
\hdashline- \\
\hdashline
\end{tabular} & \begin{tabular}{l|}
15.4 \\
17.8 \\
17.0 \\
14.7 \\
12.1 \\
16.6 \\
26 \\
22 \\
24 \\
24 \\
\\
\hdashline
\end{tabular} & 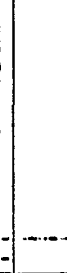 & $\begin{array}{r}11.0 \\
13.6 \\
12.3 \\
8.3 \\
8.8 \\
13.2 \\
.3 \\
8.7 \\
8.7 \\
\hdashline 3.0\end{array}$ & $\begin{array}{r}12.4 \\
15.1 \\
14.6 \\
10.8 \\
10.0 \\
9.4 \\
17.1 \\
11.0 \\
18.2 \\
13.8 \\
13.8 \\
10 .\end{array}$ & & $\begin{array}{l}19.2 \\
23.4 \\
22.4 \\
16.9 \\
15.5 \\
14.6 \\
26.5 \\
17.0 \\
28.2 \\
21.4 \\
21.5 \\
16.1\end{array}$ & $\begin{array}{l}384 \\
467 \\
435 \\
338 \\
300 \\
292 \\
532 \\
307 \\
563 \\
415 \\
431 \\
311\end{array}$ & & $\begin{array}{r}1,180 \\
1,440 \\
1,330 \\
1,040 \\
921 \\
896 \\
1,630 \\
945 \\
1,730 \\
1,270 \\
1,320 \\
957\end{array}$ \\
\hline The year & $\ldots$ & 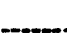 & - & 26 & & .2 & 13.1 & & 20.3 & 4,780 & & 14,700 \\
\hline
\end{tabular}


MANUEL IUIS DITCH AT PUOHOKAMOA GULCH, NEAR HUELO, MAUI

Location.-Water-stage recorder in Puohokamoa Gulch at lower portal of tunnel between Haipuaena and Puohokamoa Streams, 3 miles southeast of Kailua.

Records avaIL ABLe.-December, 1917, to June, 1929.

Extremes.- Maximum discharge during year, 100 million gallons a day or 155 second-feet Feb. 17 (gage height, 4.00 feet); minimum, 0.2 million gallons a day or 0.3 second-foot June 28-30.

1917-1929: Maximum discharge, 116 million gallons a day or 179 secondfeet Jan. 14, 1923 (gage height, 4.93 feet); minimum, 0.05 million gallons a day or 0.08 second-foot Mar. 3, 1920.

REMARKS.- Records good for low stages and fair above; estimated records poor. Ditch is extension of Center ditch and picks up water at elevation of 500 feet between Kolea and Waikamoi Streams. Regulated by gates.

Discharge, in million gallons a day, 1928-29

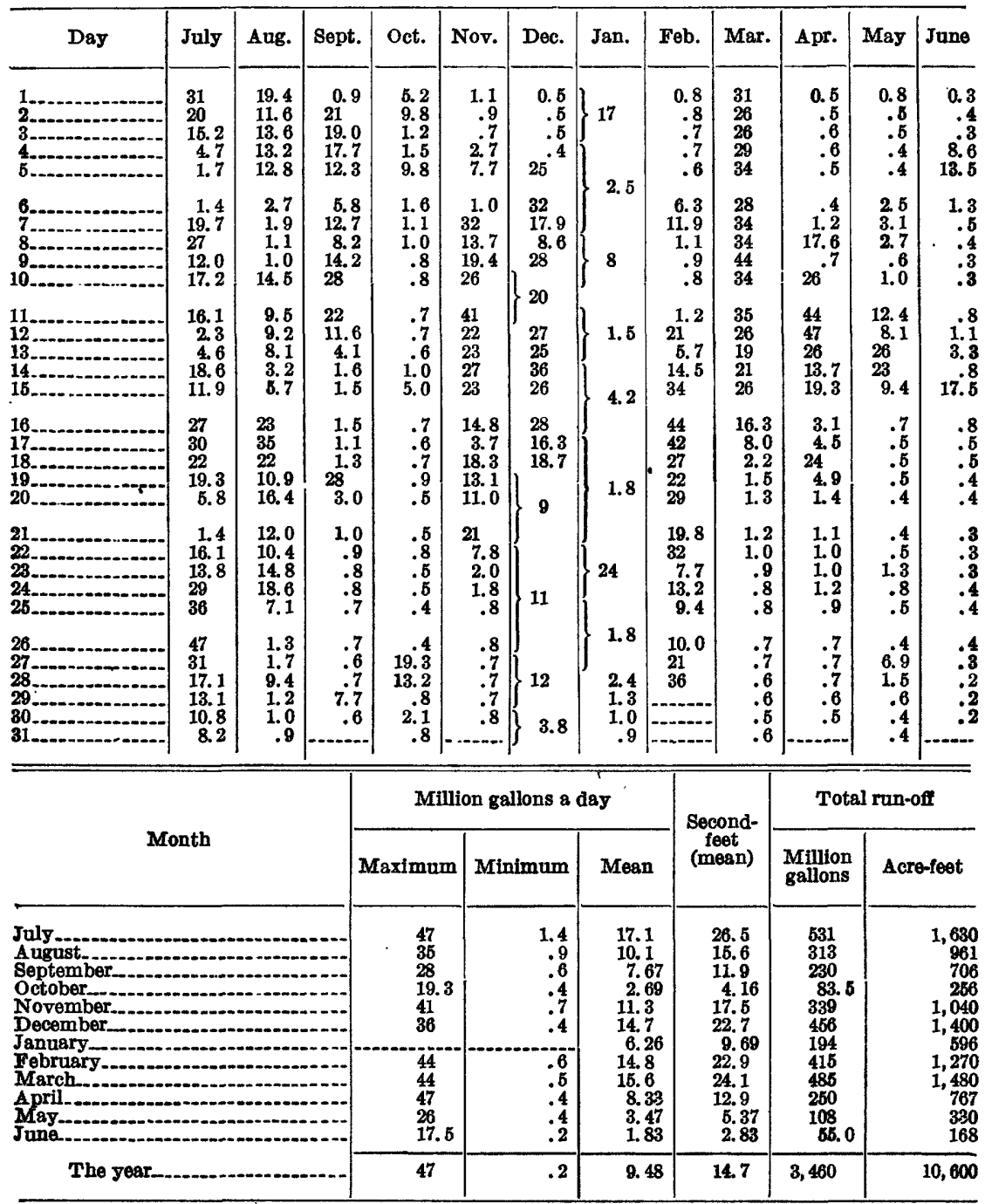


SPRECKELS DITCH AT WAHINEPE, NEAR HUELO, MAUI

Location.-Water-stage recorder between Puohokamoa and Alo Streams, 1,000 feet below intake at Puohokamoa Gulch and 7 miles southeast of Kailua.

ReCords avatrable.-August, 1928, to June, 1929.

EXTREMES.-Maximum discharge during period, 64 million gallons a day or 99 second-feet Dec. 13 (gage height, 4.73 feet); minimum recorded, 0.1 million gallons a day or 0.2 second-foot May 2; actual minimum may have occurred during period of missing record.

Remarks.-Records good. Intake is on Puohokamoa Stream just below intake of Koolau ditch and for normal flows takes all water which passes Koolau ditch intake.

Discharge, in million gallons a day, 1928-29

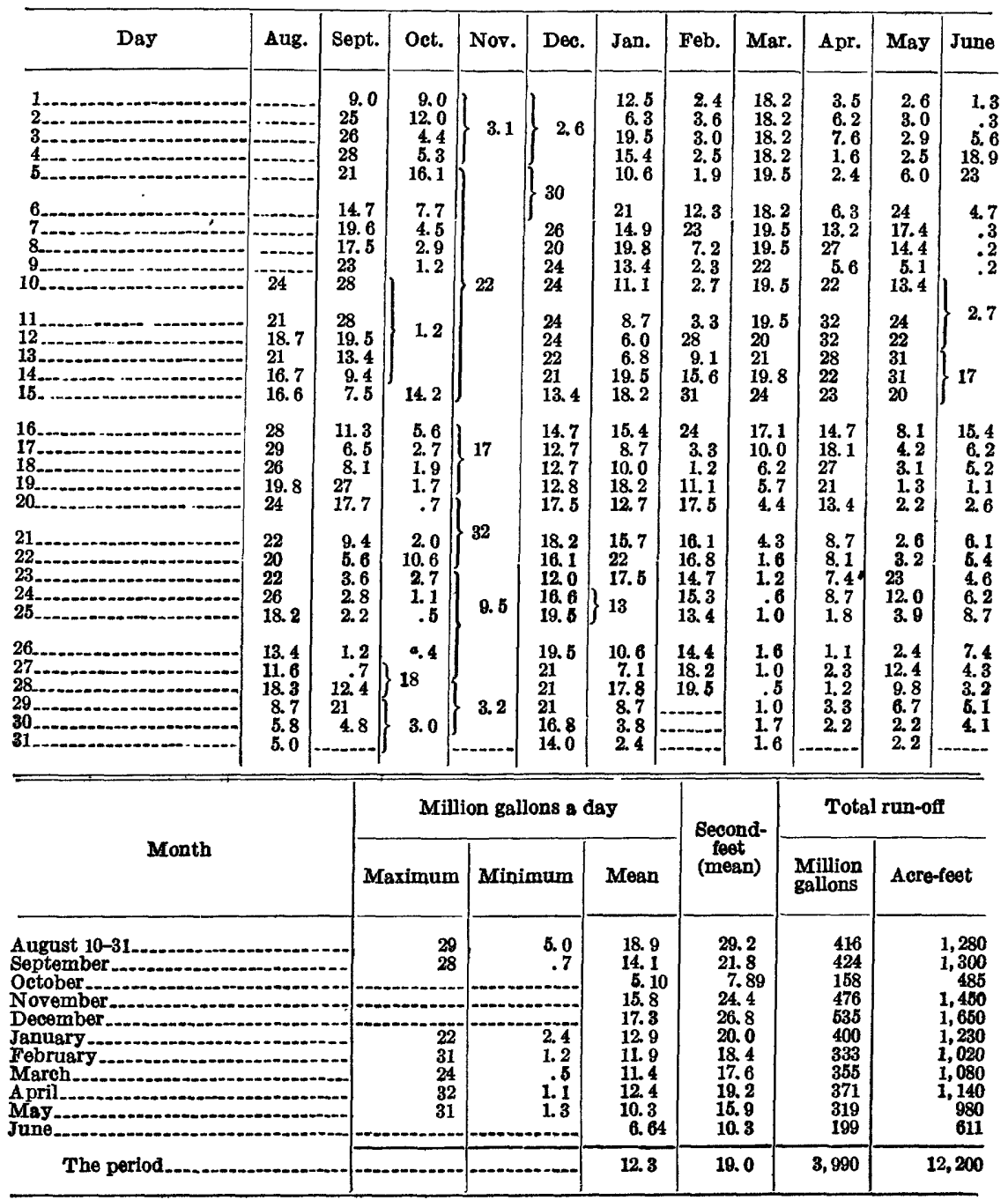

-Estimated. 


\section{KOOLAర DITCH AT WAHINEPE, NEAB\}HUELO, MAOI}

Location.-W ater-stage recorder between Puohokeımoa and Waikamoi Streams, half a mile below Puohokamoa intake and 21/2 miles southeast of Kailua.

RECORDs AVAILABLE. - March, 1922, to June, 1929.

EXTREMEs.-Maximum discharge during year nct known, owing to missing record; minimum, 1.4 million gallons a day or 2.2 second-feet Feb. 21 .

1922-1929: Maximum discharge, 123 million gallons a day or 190 secondfeet Dec. 8 (gage height, 5.62 feet); minimum, that of Feb. 21, 1929.

REMarks.-Records good except those for estimated periods, which are fair. Completely regulated by gates and spillways. Koolau ditch diverts water at 1,200-foot elevation from all streams from Makapipi to Alo.

Discharge, in million gallons a day, 1928-29

\begin{tabular}{|c|c|c|c|c|c|c|c|c|c|c|c|c|}
\hline Day & July & Aug. & Sept. & Oct. & Nov. & Dec. & Jan. & Feb. & Mar. & Apr. & May & June \\
\hline 1 & $\begin{array}{r}107 \\
104 \\
104 \\
93 \\
77\end{array}$ & $\begin{array}{l}113 \\
110 \\
110 \\
110 \\
110\end{array}$ & $\begin{array}{r}81 \\
110 \\
110 \\
110 \\
107\end{array}$ & $\begin{array}{r}68 \\
98 \\
74 \\
77 \\
104\end{array}$ & $\begin{array}{l}52 \\
49 \\
42 \\
45 \\
76\end{array}$ & 50 & 100 & $\begin{array}{l}56 \\
52 \\
49 \\
47 \\
44\end{array}$ & $\begin{array}{l}113 \\
110 \\
110 \\
113 \\
113\end{array}$ & $\begin{array}{l}46 \\
47 \\
50 \\
46 \\
39\end{array}$ & $\begin{array}{l}48 \\
36 \\
34 \\
32 \\
36\end{array}$ & $\begin{array}{r}36 \\
35 \\
29 \\
85 \\
110\end{array}$ \\
\hline \begin{tabular}{c}
6 \\
$7 \ldots \ldots$ \\
8 \\
8 \\
90 \\
10 \\
\hdashline
\end{tabular} & $\begin{array}{r}70 \\
100 \\
107 \\
101 \\
104\end{array}$ & $\begin{array}{r}104 \\
101 \\
93 \\
85 \\
110\end{array}$ & $\begin{array}{l}104 \\
107 \\
104 \\
110 \\
113\end{array}$ & $\begin{array}{l}79 \\
64 \\
62 \\
56 \\
52\end{array}$ & $\begin{array}{r}54 \\
101 \\
102 \\
104 \\
107\end{array}$ & $\begin{array}{r}99 \\
98 \\
107\end{array}$ & & $\begin{array}{l}63 \\
92 \\
62 \\
51 \\
44\end{array}$ & $\begin{array}{l}113 \\
113 \\
113 \\
116 \\
113\end{array}$ & $\begin{array}{r}44 \\
54 \\
103 \\
58 \\
97\end{array}$ & $\begin{array}{l}83 \\
83 \\
85 \\
48 \\
60\end{array}$ & $\begin{array}{l}95 \\
61 \\
44 \\
39 \\
39\end{array}$ \\
\hline 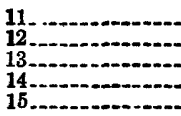 & $\begin{array}{r}101 \\
91 \\
96 \\
104 \\
99\end{array}$ & $\begin{array}{r}107 \\
107 \\
107 \\
99 \\
101\end{array}$ & $\begin{array}{r}113 \\
110 \\
105 \\
93 \\
85\end{array}$ & $\begin{array}{l}47 \\
44 \\
42 \\
52 \\
69\end{array}$ & $\begin{array}{l}110 \\
107 \\
107 \\
107 \\
106\end{array}$ & & 100 & \begin{tabular}{|r|}
47 \\
98 \\
83 \\
85 \\
104 \\
\end{tabular} & $\begin{array}{l}116 \\
113 \\
110 \\
110 \\
113\end{array}$ & $\begin{array}{l}110 \\
110 \\
110 \\
110 \\
110\end{array}$ & $\begin{array}{l}106 \\
105 \\
116 \\
116 \\
106\end{array}$ & $\begin{array}{r}61 \\
57 \\
79 \\
58 \\
109\end{array}$ \\
\hline $\begin{array}{l}16 \\
18 \\
18 \\
18\end{array}$ & $\begin{array}{l}107 \\
107 \\
104 \\
107 \\
104\end{array}$ & $\begin{array}{l}116 \\
116 \\
113 \\
107 \\
110\end{array}$ & $\begin{array}{r}85 \\
77 \\
75 \\
113 \\
97\end{array}$ & $\begin{array}{l}47 \\
44 \\
42 \\
44 \\
38\end{array}$ & $\begin{array}{r}101 \\
99 \\
104 \\
101 \\
99\end{array}$ & & 81) & \begin{tabular}{|c|}
104 \\
56 \\
13.6 \\
3.4 \\
2.7
\end{tabular} & $\begin{array}{r}110 \\
107 \\
104 \\
99 \\
93\end{array}$ & $\begin{array}{r}98 \\
90 \\
107 \\
91 \\
72\end{array}$ & $\begin{array}{l}81 \\
67 \\
59 \\
54 \\
46\end{array}$ & $\begin{array}{l}86 \\
59 \\
62 \\
47 \\
40\end{array}$ \\
\hline 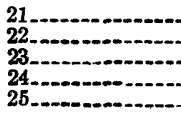 & $\begin{array}{r}91 \\
104 \\
110 \\
116 \\
116\end{array}$ & $\begin{array}{l}110 \\
107 \\
110 \\
113 \\
107\end{array}$ & $\begin{array}{l}80 \\
72 \\
67 \\
64 \\
62\end{array}$ & $\begin{array}{l}44 \\
58 \\
42 \\
38 \\
37\end{array}$ & $\begin{array}{r}104 \\
99 \\
93 \\
88 \\
77\end{array}$ & & 10) & $\begin{array}{r}2.2 \\
2.5 \\
2.7 \\
36 \\
107\end{array}$ & $\begin{array}{l}87 \\
80 \\
75 \\
72 \\
67\end{array}$ & $\begin{array}{l}64 \\
62 \\
59 \\
60 \\
54\end{array}$ & $\begin{array}{l}70 \\
43 \\
81 \\
79 \\
48\end{array}$ & $\begin{array}{l}35 \\
32 \\
31 \\
82 \\
34\end{array}$ \\
\hline $\begin{array}{l}26 \\
28 \\
28 \\
30\end{array}$ & $\begin{array}{l}119 \\
116 \\
113 \\
110 \\
110 \\
107\end{array}$ & $\begin{array}{r}99 \\
100 \\
83 \\
75 \\
70\end{array}$ & $\begin{array}{l}59 \\
56 \\
67 \\
97 \\
62\end{array}$ & $\begin{array}{l}35 \\
67 \\
94 \\
58 \\
62 \\
58\end{array}$ & $\begin{array}{l}72 \\
67 \\
62 \\
59 \\
62\end{array}$ & & $\begin{array}{l}93 \\
81 \\
7.2 \\
64 \\
62\end{array}$ & 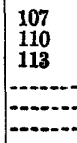 & $\begin{array}{l}62 \\
59 \\
54 \\
52 \\
47 \\
47\end{array}$ & $\begin{array}{l}54 \\
51 \\
47 \\
47 \\
44\end{array}$ & $\begin{array}{l}42 \\
75 \\
65 \\
50 \\
42 \\
38\end{array}$ & $\begin{array}{l}31 \\
28 \\
27 \\
23 \\
28\end{array}$ \\
\hline
\end{tabular}

\begin{tabular}{|c|c|c|c|c|c|c|}
\hline \multirow{2}{*}{ Month } & \multicolumn{3}{|c|}{ Million gallons a day } & \multirow{2}{*}{$\begin{array}{l}\text { Second- } \\
\text { feet } \\
\text { (mean) }\end{array}$} & \multicolumn{2}{|c|}{ Total run off } \\
\hline & Maximum & Minimum & Metan & & $\begin{array}{l}\text { Million } \\
\text { gallons }\end{array}$ & Acre-feet \\
\hline $\begin{array}{l}\text { July } \\
\text { August } \\
\text { September. } \\
\text { Ootober } \\
\text { November- } \\
\text { December } \\
\text { January } \\
\text { February } \\
\text { March } \\
\text { April } \\
\text { May }\end{array}$ & $\begin{array}{l}\mathbf{1 1 9} \\
\mathbf{1 1 6} \\
\mathbf{1 1 3} \\
\mathbf{1 0 4} \\
\mathbf{1 1 0}\end{array}$ & $\begin{array}{l}22 \\
47 \\
39 \\
32 \\
23 \\
\end{array}$ & $\begin{array}{r}103 \\
103 \\
39.8 \\
57.9 \\
35.2 \\
93.0 \\
38.4 \\
58.5 \\
78.7 \\
71.1 \\
35.6 \\
50.7\end{array}$ & $\begin{array}{c}159 \\
159 \\
139 \\
89.6 \\
132 \\
144 \\
137 \\
90.5 \\
145 \\
110 \\
101 \\
78.4\end{array}$ & $\begin{array}{l}3,200 \\
3,200 \\
2,700 \\
1,800 \\
2,560 \\
2,880 \\
2,740 \\
1,640 \\
2,900 \\
2,130 \\
2,030 \\
1,520\end{array}$ & $\begin{array}{l}9,800 \\
9,800 \\
8,270 \\
5,510 \\
7,840 \\
8,850 \\
8,410 \\
5,030 \\
8,910 \\
6,550 \\
6,240 \\
4,670\end{array}$ \\
\hline The year & & 2.2 & 30.3 & 124 & 29,300 & 89,900 \\
\hline
\end{tabular}




\section{ALO STREAM NEAR HUELO, MAUI}

Location.-Water-stage recorder just above Spreckels ditch inflow and trail crossing and $2 \frac{1}{2}$ miles southeast of Kailua.

DratNAGE AREA.- 0.2 square mile.

Records available.-December, 1910, to June, 1929.

EXTREMEs.-Maximum discharge during year, 410 million gallons a day or 634 second-feet some time between Dec. 7 and Jan. 25 (gage height, 3.75 feet); minimum, 0.5 million gallons a day or 0.8 second-foot Oct. 27 .

1910-1929: Maximum discharge, 638 million gallons a day or 987 secondfeet Dec. 9, 1916 (gage height, 4.35 feet); minimum, 0.4 million gallons a day or 0.6 second-foot Sept. 19, 1924, and Mar. 13-16, 1926.

Remarks.-Records poor. No diversions.

Discharge, in million gallons a day, 1928-29

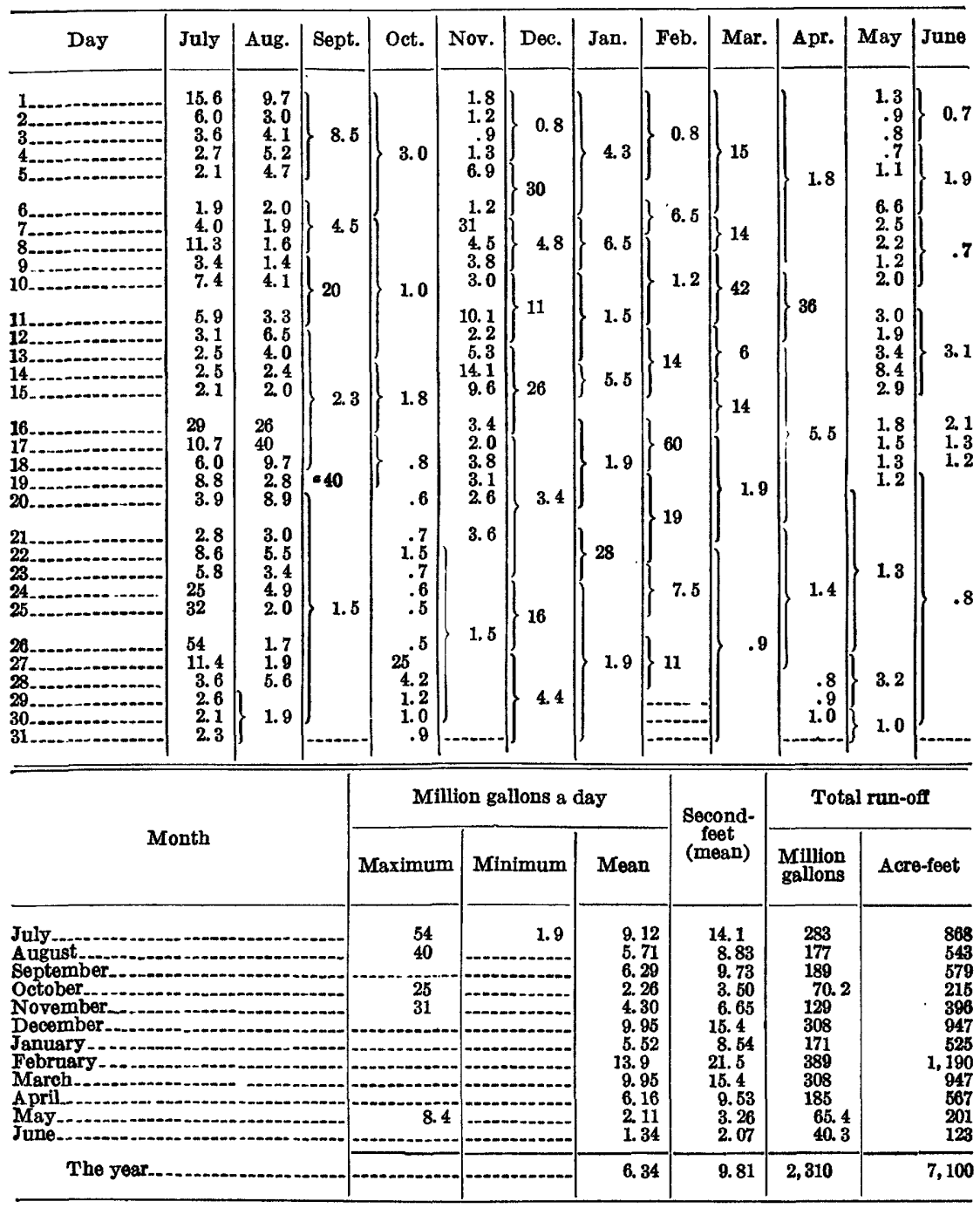

- Estimated. 
WAIKAMOI STREAM ABOVE WAHLA DITCH, NEAR HUELO, MAUI

Locatron.-Water-stage recorder 500 feet above intake of Wailoa ditch, onequarter mile above Spreckels ditch trail, and $21 / 2$ miles southeast of Kailua.

Drain AGE AREA. - 4.4 square miles.

Records avaIrable.-January, 1922, to June, 1929.

EXTREMES.-Maximum discharge during year, 863 million gallons a day or 1,340 second-feet Feb. 17 (gage height, 7.95 feet); minimum, 1.2 million gallons a day or 1.9 second-feet June 29,30 .

1922-1929: Maximum discharge, 1,360 million gallons a day or 2,100 second-feet Oct. 16, 1924 (gage height, 10.45 feet); minimum, 0.5 million gallons a day or 0.8 second-foot Mar. 21 and 22, 1926.

REMARKs.- Record good for ordinary stages; poor for high stages. Haleakala ranch and Kula pipe lines divert small amounts of water above station.

Discharge, in million gallons a day, 1928-29

\begin{tabular}{|c|c|c|c|c|c|c|c|c|c|c|c|c|}
\hline Day & July & Aug. & Sept. & Oct. & Nov. & Dec. & Jan. & Feb. & Mar. & Apr. & May & June \\
\hline $\begin{array}{l}1 \\
2 \\
3 \\
4 \\
5 \\
5\end{array}$ & \begin{tabular}{r}
\multicolumn{1}{c}{64} \\
12.3 \\
9.7 \\
7.6 \\
5.7
\end{tabular} & $\begin{array}{r}12.6 \\
11.1 \\
14.2 \\
9.8 \\
9.9\end{array}$ & $\begin{array}{c}4.9 \\
48 \\
25 \\
24 \\
9.5\end{array}$ & $\begin{array}{l}7.8 \\
5.7 \\
4.0 \\
4.0 \\
6.7\end{array}$ & $\begin{array}{r}3.4 \\
3.4 \\
2.9 \\
3.2 \\
12.3\end{array}$ & $\begin{array}{r}3.0 \\
2.8 \\
2.8 \\
2.6 \\
75\end{array}$ & $\begin{array}{c}20 \\
7.8 \\
45 \\
10.6 \\
7.6\end{array}$ & $\begin{array}{l}3.8 \\
3.5 \\
3.3 \\
3.0 \\
2.8\end{array}$ & $\begin{array}{l}49 \\
29 \\
26 \\
35 \\
50\end{array}$ & $\begin{array}{l}2.1 \\
2.7 \\
2.8 \\
2.4 \\
2.0\end{array}$ & $\begin{array}{l}2.8 \\
2.0 \\
1.8 \\
1.6 \\
2.1\end{array}$ & $\begin{array}{l}2.4 \\
2.2 \\
2.0 \\
22 \\
28\end{array}$ \\
\hline $\begin{array}{r}6 \\
7 \\
8 \\
9 \\
10\end{array}$ & $\begin{array}{r}4.8 \\
18.0 \\
28 \\
11.1 \\
11.8\end{array}$ & $\begin{array}{r}6.6 \\
6.3 \\
5.6 \\
4.8 \\
19.2\end{array}$ & $\begin{array}{r}6.7 \\
11.6 \\
7.8 \\
10.0 \\
65\end{array}$ & $\begin{array}{l}4.8 \\
3.9 \\
3.5 \\
3.0 \\
2.8\end{array}$ & \begin{tabular}{l}
\multicolumn{4}{c}{.6} \\
81 \\
16.9 \\
33 \\
56
\end{tabular} & $\begin{array}{l}69 \\
25 \\
17.3 \\
49 \\
43\end{array}$ & $\begin{array}{l}22 \\
11.0 \\
38 \\
12.0 \\
7.1\end{array}$ & $\begin{array}{r}6.5 \\
19.9 \\
6.4 \\
4.0 \\
3.3\end{array}$ & $\begin{array}{l}38 \\
71 \\
52 \\
90 \\
43\end{array}$ & $\begin{array}{c}2.8 \\
5.0 \\
21 \\
5.0 \\
57\end{array}$ & $\begin{array}{r}10.9 \\
12.7 \\
12.0 \\
5.2 \\
6.2\end{array}$ & $\begin{array}{r}11.5 \\
6.1 \\
3.5 \\
2.7 \\
2.6\end{array}$ \\
\hline $\begin{array}{l}11 \\
13 \\
15\end{array}$ & $\begin{array}{r}9.9 \\
7.2 \\
9.9 \\
24 \\
13.9\end{array}$ & $\begin{array}{r}13.4 \\
9.2 \\
9.1 \\
8.9 \\
8.0\end{array}$ & $\begin{array}{l}33 \\
9.5 \\
6.6 \\
5.4 \\
4.9\end{array}$ & $\begin{array}{l}2.6 \\
2.4 \\
2.3 \\
3.2 \\
7.4\end{array}$ & $\begin{array}{r}120 \\
32 \\
30 \\
39 \\
29\end{array}$ & $\begin{array}{r}31 \\
63 \\
62 \\
104 \\
46\end{array}$ & $\begin{array}{r}5.7 \\
4.9 \\
4.9 \\
56 \\
19.6\end{array}$ & $\begin{array}{c}3.4 \\
21 \\
7.6 \\
12.8 \\
65\end{array}$ & $\begin{array}{l}50 \\
20 \\
12.5 \\
17.2 \\
20\end{array}$ & $\begin{array}{c}114 \\
152 \\
40 \\
15.2 \\
21\end{array}$ & $\begin{array}{l}21 \\
13.2 \\
61 \\
27 \\
13.6\end{array}$ & $\begin{array}{r}5.1 \\
4.0 \\
8.0 \\
4.8 \\
39\end{array}$ \\
\hline $\begin{array}{l}16 \\
17 \\
18 \\
19\end{array}$ & $\begin{array}{l}59 \\
34 \\
14.7 \\
13.6 \\
10.3\end{array}$ & $\begin{array}{l}25 \\
66 \\
31 \\
10.1 \\
13.9\end{array}$ & $\begin{array}{r}5.7 \\
4.9 \\
6.2 \\
54 \\
9.6\end{array}$ & $\begin{array}{l}3.7 \\
2.8 \\
2.7 \\
2.6 \\
2.3\end{array}$ & $\begin{array}{r}11.1 \\
7.9 \\
13.3 \\
11.8 \\
31\end{array}$ & $\begin{array}{r}31 \\
13.9 \\
14.5 \\
9.3 \\
10.8\end{array}$ & $\begin{array}{r}10.1 \\
6.4 \\
5.8 \\
17.8 \\
9.1\end{array}$ & $\begin{array}{r}155 \\
278 \\
94 \\
41 \\
171\end{array}$ & $\begin{array}{l}9.1 \\
7.0 \\
5.6 \\
5.5 \\
5.4\end{array}$ & $\begin{array}{c}9.8 \\
7.4 \\
35 \\
9.8 \\
6.2\end{array}$ & $\begin{array}{l}7.2 \\
5.4 \\
4.6 \\
3.8 \\
3.1\end{array}$ & $\begin{array}{l}8.5 \\
4.6 \\
4.8 \\
3.3 \\
2.7\end{array}$ \\
\hline 23 & $\begin{array}{l}7.6 \\
29 \\
13.3 \\
67 \\
89\end{array}$ & $\begin{array}{r}16.5 \\
9.7 \\
16.3 \\
29 \\
9.9\end{array}$ & $\begin{array}{l}5.8 \\
4.8 \\
4.0 \\
3.7 \\
3.4\end{array}$ & $\begin{array}{l}2.6 \\
4.2 \\
2.9 \\
2.4 \\
2.2\end{array}$ & $\begin{array}{c}25 \\
8.1 \\
6.3 \\
5.8 \\
8.7\end{array}$ & $\begin{array}{r}7.8 \\
6.6 \\
5.4 \\
7.1 \\
56\end{array}$ & $\begin{array}{c}6.2 \\
168 \\
54 \\
14.4 \\
8.9\end{array}$ & $\begin{array}{c}136 \\
106 \\
27 \\
19.9 \\
10.5\end{array}$ & $\begin{array}{l}\text { 4. } 6 \\
\text { 3. } 6 \\
\text { 3. } 3 \\
3.0 \\
2.9\end{array}$ & $\begin{array}{l}4.8 \\
4.3 \\
4.0 \\
4.0 \\
3.1\end{array}$ & $\begin{array}{r}2.8 \\
2.9 \\
10.2 \\
8.9 \\
4.0\end{array}$ & $\begin{array}{l}2.3 \\
2.1 \\
1.9 \\
2.2 \\
3.0\end{array}$ \\
\hline $\begin{array}{l}26 \\
27 \\
29 \\
29 \\
31\end{array}$ & $\begin{array}{r}163 \\
74 \\
19.8 \\
11.5 \\
9.1 \\
9.2\end{array}$ & $\begin{array}{l}7.1 \\
6.3 \\
8.0 \\
5.1 \\
4.5 \\
4.2\end{array}$ & $\begin{array}{r}3.1 \\
2.9 \\
7.6 \\
17.1 \\
5.6 \\
\end{array}$ & $\begin{array}{r}2.0 \\
54.0 \\
17.2 \\
5.3 \\
3.7 \\
3.3\end{array}$ & $\begin{array}{l}7.1 \\
4.6 \\
3.9 \\
3.6 \\
3.5 \\
\\
\end{array}$ & $\begin{array}{l}42 \\
11.3 \\
20 \\
16.0 \\
7.9 \\
6.1\end{array}$ & $\begin{array}{r}7.1 \\
5.8 \\
11.6 \\
8.3 \\
5.4 \\
4.3\end{array}$ & $\begin{array}{l}11.9 \\
29 \\
93 \\
\end{array}$ & $\begin{array}{l}2.6 \\
2.4 \\
2.2 \\
2.0 \\
1.9 \\
1.8\end{array}$ & $\begin{array}{l}2.8 \\
2.8 \\
2.4 \\
3.3 \\
2.9\end{array}$ & $\begin{array}{l}2.8 \\
5.7 \\
7.7 \\
7.6 \\
3.4 \\
2.8\end{array}$ & $\begin{array}{l}2.6 \\
1.7 \\
1.4 \\
1.3 \\
1.4\end{array}$ \\
\hline
\end{tabular}

\begin{tabular}{|c|c|c|c|c|c|c|}
\hline \multirow{2}{*}{ Month } & \multicolumn{3}{|c|}{ Million gallons a day } & \multirow{2}{*}{$\begin{array}{l}\text { Second- } \\
\text { feet } \\
\text { (mean) }\end{array}$} & \multicolumn{2}{|c|}{ Total run-off } \\
\hline & Maximum & Minimum & Mean & & $\begin{array}{l}\text { Million } \\
\text { gallons }\end{array}$ & Acre-feet \\
\hline $\begin{array}{l}\text { July } \\
\text { Angust } \\
\text { September } \\
\text { October } \\
\text { November. } \\
\text { December } \\
\text { January } \\
\text { February } \\
\text { March } \\
\text { April }\end{array}$ & $\begin{array}{r}163 \\
66 \\
65 \\
54 \\
120 \\
104 \\
168 \\
278 \\
90 \\
152 \\
61 \\
39\end{array}$ & $\begin{array}{l}4.8 \\
4.2 \\
2.9 \\
2.0 \\
2.9 \\
2.6 \\
4.3 \\
2.8 \\
1.8 \\
2.0 \\
1.6 \\
1.3\end{array}$ & $\begin{array}{r}27.8 \\
13.3 \\
13.7 \\
5.74 \\
20.6 \\
27.8 \\
19.9 \\
47.8 \\
21.5 \\
18.3 \\
8.90 \\
6.26\end{array}$ & $\begin{array}{l}43.0 \\
20.6 \\
21.2 \\
8.88 \\
31.9 \\
43.0 \\
30.8 \\
74.0 \\
33.3 \\
28.3 \\
13.8 \\
9.69\end{array}$ & $\begin{array}{r}862 \\
411 \\
410 \\
178 \\
618 \\
861 \\
615 \\
1,340 \\
666 \\
548 \\
276 \\
188\end{array}$ & $\begin{array}{r}2,640 \\
1,270 \\
1,260 \\
546 \\
1,900 \\
2,640 \\
1,890 \\
4,110 \\
2,050 \\
1,680 \\
847 \\
576\end{array}$ \\
\hline The year. & 278 & 1.3 & 19.1 & 29.6 & 6,970 & 21,400 \\
\hline
\end{tabular}




\section{KAAIRA STREAM NEAR KAIIUA, MAUI}

Location.-Water-stage recorder 700 feet above Hamakua ditch trail crossing and 2 miles southeast of Kailua.

Drain AGE AREA. - 0.5 square mile.

RECORDS AVAILABLE.-December, 1921, to June, 1929.

Extremes.-Maximum discharge during year, 504 million gallons a day or 780 second-feet Dec. 13 (gage height, 3.52 feet); minimum, 0.6 million gallons a day or 0.9 second-foot May 4.

1921-1929: Maximum discharge, 900 million gallons a day or 1,390 secondfeet Jan. 31, 1922 (gage height, 4.92 feet); minimum, 0.3 million gallons a day or 0.5 second-foot July 17, 1922, and Mar. 22, 1927.

REMA Ks.-Records good for ordinary stages and poor for high stages. No diversions.

Discharge, in million gallons a day, 1928-29

\begin{tabular}{|c|c|c|c|c|c|c|c|c|c|c|c|c|}
\hline Day & July & Aug. & Sept. & Oct. $N$ & Nov. & Dec. & Jan. & Feb. & Mar. & Apr. & May & June \\
\hline & $\begin{array}{l}4.2 \\
3.0 \\
2.1 \\
1.8\end{array}$ & $\begin{array}{l}7.7 \\
3.1 \\
4.5 \\
4.8 \\
4.9\end{array}$ & $\begin{array}{r}1.5 \\
23 \\
6.8 \\
4.7 \\
2.8\end{array}$ & $\begin{array}{l}1.4 \\
1.9 \\
4.4\end{array}$ & $\begin{array}{l}1.8 \\
1.4 \\
1.1 \\
2.5 \\
5.8\end{array}$ & $\begin{array}{r}0.9 \\
.9 \\
.9 \\
.9 \\
39\end{array}$ & $\begin{array}{l}7.0 \\
2.2 \\
3.7 \\
3.2 \\
2.4\end{array}$ & \begin{tabular}{r|}
1.0 \\
.9 \\
.9 \\
.8 \\
.7
\end{tabular} & $\begin{array}{l}13.4 \\
32\end{array}$ & $\begin{array}{r}0.9 \\
1.1 \\
1.8 \\
1.2 \\
.8\end{array}$ & $\begin{array}{r}1.4 \\
.7 \\
.7 \\
.6 \\
.9\end{array}$ & 3. \\
\hline $\begin{array}{l}7 \\
9\end{array}$ & $\begin{array}{l}1.5 \\
2.9 \\
7.7 \\
2.8 \\
5.5\end{array}$ & $\begin{array}{l}2.6 \\
2.4 \\
1.9 \\
1.6 \\
4.3\end{array}$ & $\begin{array}{r}2.2 \\
7.1 \\
2.6 \\
8.6 \\
33\end{array}$ & \begin{tabular}{l|}
2.1 \\
1.6 \\
1.4 \\
1.2 \\
1.0
\end{tabular} & \begin{tabular}{r|}
1.5 \\
26 \\
4.3 \\
3.5 \\
3.9
\end{tabular} & $\begin{array}{r}9.1 \\
3.4 \\
5.4 \\
7.9 \\
15.0\end{array}$ & \begin{tabular}{r|}
6.2 \\
3.0 \\
12.9 \\
3.0 \\
2.2
\end{tabular} & $\begin{array}{l}5.6 \\
5.5 \\
1.6 \\
1.1 \\
1.1\end{array}$ & $\begin{array}{r}7.2 \\
11.6 \\
12.2 \\
68 \\
16.6\end{array}$ & $\begin{array}{r}1.0 \\
1.9 \\
6.4 \\
1.4 \\
32 .\end{array}$ & $\begin{array}{l}6.5 \\
3.4 \\
2.8 \\
1.3 \\
2.3\end{array}$ & 1. \\
\hline | & $\begin{array}{l}4.4 \\
2.6 \\
2.1 \\
3.2 \\
1.9\end{array}$ & $\begin{array}{l}3.5 \\
5.2 \\
\text { 3. } 6 \\
3.2 \\
2.6\end{array}$ & $\begin{array}{r}11.0 \\
3.5 \\
2.4 \\
1.9 \\
1.8\end{array}$ & $\begin{array}{r}.9 \\
.9 \\
.8 \\
1.7 \\
2.8\end{array}$ & \begin{tabular}{r|}
9.3 \\
2.8 \\
5.6 \\
16.7 \\
6.0
\end{tabular} & $\begin{array}{c}8.1 \\
9.7 \\
37 \\
29 \\
8.5\end{array}$ & $\begin{array}{l}1.9 \\
1.5 \\
1.5 \\
7.3 \\
3.5\end{array}$ & $\begin{array}{r}1.5 \\
11.2 \\
2.1 \\
11.6 \\
24\end{array}$ & $\begin{array}{c}25 \\
5.7 \\
3.7 \\
7.6 \\
21\end{array}$ & $\begin{array}{l}40 \\
26 \\
8.3 \\
3.2 \\
3.3\end{array}$ & $\begin{array}{l}4.3 \\
2.8 \\
4.8 \\
8.6 \\
3.2\end{array}$ & 1. \\
\hline (n........ & $\begin{array}{c}25 \\
5.0 \\
4.8 \\
5.9 \\
3.2\end{array}$ & $\begin{array}{l}21 \\
31 \\
9.1 \\
3.0 \\
6.0\end{array}$ & $\begin{array}{r}2.2 \\
1.6 \\
1.7 \\
33 \\
2.8\end{array}$ & $\begin{array}{r}1.1 \\
1.0 \\
.8 \\
.9 \\
.7\end{array}$ & $\begin{array}{l}3.5 \\
2.4 \\
4.4 \\
3.4 \\
3.9\end{array}$ & $\begin{array}{r}19.1 \\
4.2 \\
7.0 \\
3.7 \\
2.8\end{array}$ & $\begin{array}{l}2.4 \\
1.6 \\
1.6 \\
2.8 \\
1.8\end{array}$ & $\begin{array}{l}62 \\
55 \\
38 \\
21 \\
25\end{array}$ & $\begin{array}{l}3.7 \\
2.8 \\
2.2 \\
2.1 \\
1.9\end{array}$ & \begin{tabular}{r|}
2.6 \\
2.8 \\
21 \\
3.2 \\
2.2
\end{tabular} & $\begin{array}{l}2.1 \\
1.8 \\
1.4 \\
1.2 \\
1.1\end{array}$ & $\begin{array}{l}2 . \\
1 . \\
1 . \\
1 . \\
1 .\end{array}$ \\
\hline 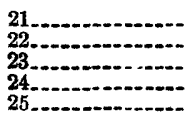 & $\begin{array}{r}2.4 \\
7.6 \\
4.1 \\
18.6 \\
21\end{array}$ & $\begin{array}{l}2.9 \\
3.9 \\
3.5 \\
4.2 \\
2.2\end{array}$ & $\begin{array}{l}1.9 \\
1.6 \\
1.4 \\
1.3 \\
1.2\end{array}$ & $\begin{array}{r}1.0 \\
2.0 \\
1.0 \\
.8 \\
.7\end{array}$ & $\begin{array}{l}4.2 \\
2.1 \\
1.8 \\
1.9 \\
2.2\end{array}$ & $\begin{array}{r}3.2 \\
2.8 \\
2.2 \\
4.5 \\
30\end{array}$ & \begin{tabular}{r|}
3.3 \\
69 \\
8.5 \\
3.0 \\
2.1
\end{tabular} & $\begin{array}{r}11.7 \\
21.0 \\
5.0 \\
14.7 \\
2.8\end{array}$ & $\begin{array}{l}1.9 \\
1.4 \\
1.3 \\
1.2 \\
1.2\end{array}$ & $\begin{array}{l}1.8 \\
1.6 \\
1.5 \\
1.9 \\
1.3\end{array}$ & $\begin{array}{l}1.0 \\
1.0 \\
3.0 \\
1.8 \\
1.2\end{array}$ & 1. \\
\hline (3) & $\begin{array}{c}43 \\
13.3 \\
4.0 \\
2.8 \\
2.4 \\
2.4\end{array}$ & $\begin{array}{l}2.1 \\
2.5 \\
4.4 \\
1.8 \\
1.6 \\
1.5\end{array}$ & $\begin{array}{l}1.0 \\
1.0 \\
1.1 \\
2.3 \\
1.0\end{array}$ & $\begin{array}{r}28.7 \\
4.5 \\
1.6 \\
1.3 \\
1.1\end{array}$ & $\begin{array}{l}1.8 \\
1.3 \\
1.2 \\
1.1 \\
1.1\end{array}$ & $\begin{array}{l}7.6 \\
3.2 \\
5.2 \\
7.0 \\
2.8 \\
2.2\end{array}$ & \begin{tabular}{l|l}
1.8 & \\
1.5 \\
3.8 \\
1.6 \\
1.2 \\
1.1 & -
\end{tabular} & $\begin{array}{c}2.9 \\
4.3 \\
23\end{array}$ & $\begin{array}{r}1.0 \\
.9 \\
.8 \\
.8 \\
.7\end{array}$ & $\begin{array}{r}1.2 \\
1.3 \\
1.0 \\
.8 \\
.9 \\
\hdashline-.\end{array}$ & $\begin{array}{l}.9 \\
6.5 \\
2.6 \\
1.5 \\
1.1 \\
1.0\end{array}$ & ? \\
\hline \multirow{2}{*}{\multicolumn{4}{|c|}{ Month }} & \multicolumn{4}{|c|}{ Million gaHons a day } & \multirow{2}{*}{\multicolumn{2}{|c|}{$\begin{array}{l}\text { Second- } \\
\text { feet } \\
\text { (mean) }\end{array}$}} & \multicolumn{3}{|c|}{ Total run-of } \\
\hline & & & & Maximum & Min & imum & Mean & & & $\begin{array}{l}\text { Million } \\
\text { gallons }\end{array}$ & Acr & 3-feet \\
\hline $\mathrm{y}$ & $1 \cdot$ & 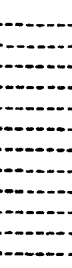 & $=$ & $\begin{array}{c}43 \\
31 \\
33 \\
28 \\
26 \\
39 \\
59 \\
62 \\
68 \\
40 \\
8.6 \\
7.3\end{array}$ & \multicolumn{2}{|c|}{$\begin{array}{r}1.5 \\
1.5 \\
1.0 \\
.7 \\
1.1 \\
.9 \\
1.7 \\
.7 \\
.8 \\
.6\end{array}$} & $\begin{array}{r}7.06 \\
5.05 \\
5.80 \\
2.45 \\
4.28 \\
9.14 \\
5.12 \\
12.7 \\
9.07 \\
5.71 \\
2.37 \\
1.48\end{array}$ & \multicolumn{2}{|c|}{$\begin{array}{c}10.9 \\
7.81 \\
8.66 \\
3.79 \\
6.62 \\
14.1 \\
7.92 \\
19.6 \\
14.0 \\
8.83 \\
3.67 \\
2.29\end{array}$} & $\begin{array}{l}219 \\
157 \\
168 \\
75.9 \\
128 \\
283 \\
159 \\
356 \\
281 \\
171 \\
73.5 \\
44.4\end{array}$ & & $\begin{array}{r}672 \\
480 \\
516 \\
233 \\
394 \\
870 \\
487 \\
1,090 \\
863 \\
526 \\
225 \\
136\end{array}$ \\
\hline The year. & & & & 68 & & .6 & 5.80 & & 8. 97 & 2,120 & & 6,490 \\
\hline
\end{tabular}




\section{SPRECKEIS DITCH BELOW KAAIRA GULCH, NEAR HUELO, MA UI}

Location.-Water-stage recorder 1,000 feet below intake in Kaaiea Stream and 1.5 miles southeast of Kailua.

Records AVAil ABLE.-December, 1917, to June, 1929.

ExTREMEs.- Maximum discharge during year, 81 million gallons a day or 125 second-feet Dec. 13 (gage height, 4.43 feet); minimum occurred sometime during periods of missing record.

1917-1929: Maximum discharge, 110 million gallons a day or 170 secondfeet Jan. 16, 1921 (gage height, 5.65 feet) and May 16, 1924 (gage height, 5.45 feet); no flow occasionally when water is turned out of ditch.

REMarKs.-Records fair except those for estimated periods, which are poor. Spreckels ditch diverts water for irrigation from all streams between Nuaailua and Kailua above Koolau ditch east of Puohokamoa and below Koolau ditch west of Puohokamoa.

Discharge, in million gallons a day, 1928-29

\begin{tabular}{|c|c|c|c|c|c|c|c|c|c|c|c|c|}
\hline Day & July & Aug. & Sept. & Oct. I & Nov. & Dec. & Jan. & Feb. & Mar. & Apr. & May & June \\
\hline $\begin{array}{l}2 \ldots \\
3-\ldots \\
4 \ldots \\
5-\ldots\end{array}$ & $\begin{array}{l}8.8 \\
3.0 \\
2.0 \\
1.5 \\
1.3\end{array}$ & $\begin{array}{l}3.1 \\
1.3 \\
2.8 \\
1.3 \\
2.8\end{array}$ & $\begin{array}{r}0.7 \\
12.1 \\
3.4 \\
1.7 \\
1.1\end{array}$ & $\begin{array}{r}5.4 \\
3.3 \\
.8 \\
.6 \\
1.3\end{array}$ & $\begin{array}{r}1.2 \\
.8 \\
.5 \\
2.5 \\
1.6\end{array}$ & $\begin{array}{r}0.3 \\
.2 \\
.2 \\
.2 \\
18.4\end{array}$ & $\begin{array}{r}6.3 \\
3.7 \\
3.3 \\
.7 \\
.7\end{array}$ & $\begin{array}{r}0.9 \\
.6 \\
.6 \\
.5 \\
.5\end{array}$ & $\begin{array}{r}7 \\
16\end{array}$ & \multirow[t]{2}{*}{0.6} & $\begin{array}{r}0.4 \\
.2 \\
.2 \\
.1 \\
.1\end{array}$ & 0.3 \\
\hline 8 & $\begin{array}{l}1.1 \\
1.5 \\
2.9 \\
1.2 \\
1.9\end{array}$ & $\begin{array}{l}1.2 \\
1.2 \\
1.0 \\
.9 \\
1.3\end{array}$ & $\begin{array}{r}1.0 \\
7.4 \\
2.3 \\
3.5 \\
13.6\end{array}$ & $\begin{array}{l}.7 \\
.6 \\
.6 \\
.6 \\
.5\end{array}$ & \begin{tabular}{l|}
.6 \\
9.9 \\
1.6 \\
1.3 \\
1.0
\end{tabular} & $\begin{array}{l}3.7 \\
1.6 \\
2.2 \\
3.7 \\
6.2\end{array}$ & $\begin{array}{r}1.0 \\
13.9 \\
1.3 \\
4.8\end{array}$ & \begin{tabular}{r|}
5.3 \\
1.9 \\
.7 \\
.5 \\
.4
\end{tabular} & 17 & & $\begin{array}{r}2.1 \\
.7 \\
. .8 \\
.5\end{array}$ & .3 \\
\hline 10 & $\begin{array}{r}2.2 \\
1.1 \\
.9 \\
.9 \\
1.2\end{array}$ & $\begin{array}{r}2.0 \\
3.3 \\
2.0 \\
1.4 \\
.9\end{array}$ & $\begin{array}{l}9.6 \\
2.7 \\
2.0 \\
1.5 \\
1.3\end{array}$ & $\begin{array}{l}.5 \\
.4 \\
.4 \\
.4 \\
.9\end{array}$ & $\begin{array}{l}5.2 \\
1.0 \\
2.5 \\
6.1 \\
2.7\end{array}$ & $\begin{array}{r}4.2 \\
7.5 \\
18.7 \\
14.5 \\
5.3\end{array}$ & $\begin{array}{r}1.6 \\
.6 \\
7.6 \\
7.0 \\
1.7\end{array}$ & $\begin{array}{l}1.4 \\
3.4 \\
4.2\end{array}$ & $\begin{array}{c}3.2 \\
10\end{array}$ & 2.8 & 1.9 & 1.7 \\
\hline $\begin{array}{l}16 \\
17 \\
19 \\
20\end{array}$ & $\begin{array}{r}10.3 \\
3.4 \\
2.0 \\
1.9 \\
1.0\end{array}$ & $\begin{array}{r}6.9 \\
17.0 \\
5.9 \\
1.3 \\
3.0\end{array}$ & $\begin{array}{r}1.1 \\
1.0 \\
1.0 \\
11.3 \\
1.0\end{array}$ & $\begin{array}{l}.4 \\
.4 \\
.4 \\
.9 \\
.4\end{array}$ & \begin{tabular}{l|}
1.3 \\
1.3 \\
3.3 \\
1.3 \\
1.4
\end{tabular} & \begin{tabular}{r|}
18.3 \\
3.6 \\
3.3 \\
2.2 \\
1.6
\end{tabular} & $\begin{array}{r}1.0 \\
.6 \\
.8 \\
1.3 \\
.7\end{array}$ & 22 & $\begin{array}{l}2.4 \\
1.7\end{array}$ & 1.4 & .4 & $\begin{array}{l}.4 \\
.4 \\
.2 \\
.2\end{array}$ \\
\hline 221232 & $\begin{array}{r}.8 \\
2.0 \\
1.9 \\
11.9 \\
13.2\end{array}$ & $\begin{array}{l}1.3 \\
2.3 \\
1.5 \\
2.2 \\
1.3\end{array}$ & $\begin{array}{r}.8 \\
1.4 \\
.6 \\
.5 \\
.4\end{array}$ & $\begin{array}{l}.4 \\
.5 \\
.4 \\
.3 \\
.3 \\
.2\end{array}$ & \begin{tabular}{r|}
1.5 \\
.9 \\
.8 \\
.7 \\
.6
\end{tabular} & $\begin{array}{r}1.8 \\
1.3 \\
1.2 \\
2.5 \\
12.3\end{array}$ & \begin{tabular}{c|}
2.8 \\
26 \\
6.1 \\
1.6 \\
1.2
\end{tabular} & 9.5 & .6 & 1.3 & 1.8 & $\begin{array}{l}.2 \\
.2 \\
.2\end{array}$ \\
\hline $\begin{array}{l}26 \\
27 \\
28 \\
30 \\
31\end{array}$ & $\begin{array}{r}19.6 \\
8.6 \\
2.6 \\
1.5 \\
1.3 \\
1.3\end{array}$ & $\begin{array}{r}1.2 \\
1.1 \\
2.5 \\
1.0 \\
.9 \\
.7\end{array}$ & $\begin{array}{l}.4 \\
.4 \\
.4 \\
.5 \\
.4\end{array}$ & $\begin{array}{r}1.2 \\
2.7 \\
.6 \\
.6 \\
.5\end{array}$ & $\begin{array}{l}.6 \\
.4 \\
.4 \\
.4 \\
.4 \\
-. .-\end{array}$ & $\begin{array}{l}3.8 \\
1.4 \\
3.4 \\
6.8 \\
1.2 \\
1.4\end{array}$ & $\begin{array}{r}.9 \\
.8 \\
3.1 \\
.9 \\
.6 \\
.6\end{array}$ & 1.7 & .4 & $\begin{array}{l}.4 \\
.2 \\
.2\end{array}$ & $\begin{array}{r}3.2 \\
.7\end{array}$ & .3 \\
\hline \multirow{2}{*}{\multicolumn{4}{|c|}{ Month }} & \multicolumn{4}{|c|}{ Million gallons a day } & \multirow{2}{*}{\multicolumn{2}{|c|}{$\begin{array}{c}\text { Second- } \\
\text { feet } \\
\text { (mean) }\end{array}$}} & \multicolumn{3}{|c|}{ Total run-off } \\
\hline & & & & Maximum & Min & imum & Mean & & & $\begin{array}{l}\text { Million } \\
\text { gallons }\end{array}$ & Acre & o-feet \\
\hline \multirow[t]{2}{*}{ 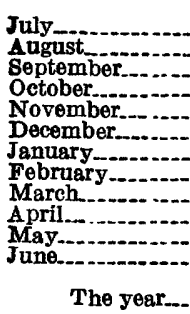 } & 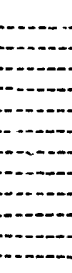 & - & \begin{tabular}{c}
$\cdots$ \\
\hdashline- \\
$\cdots-$ \\
-
\end{tabular} & $\begin{array}{l}19.6 \\
17.0 \\
13.6 \\
11.7 \\
9.9 \\
18.7 \\
26\end{array}$ & & $\begin{array}{r}0.8 \\
.7 \\
.4 \\
.2 \\
.4 \\
.2 \\
.6 \\
.4 \\
.4 \\
.2 \\
.1\end{array}$ & $\begin{array}{l}3.7 \\
2.4 \\
2.8 \\
1.2 \\
1.7 \\
4.8 \\
3.1 \\
6.9 \\
5.5 \\
3.7 \\
1.0 \\
.6\end{array}$ & \multicolumn{2}{|r|}{$\begin{array}{r}5.72 \\
3.82 \\
4.39 \\
1.87 \\
2.77 \\
7.64 \\
4.81 \\
10.8 \\
8.62 \\
5.76 \\
1.61 \\
.93\end{array}$} & $\begin{array}{c}115 \\
76.6 \\
85.1 \\
37.6 \\
53.8 \\
153 \\
96.3 \\
195 \\
173 \\
112 \\
32.3 \\
17.9\end{array}$ & \multicolumn{2}{|r|}{$\begin{array}{r}352 \\
235 \\
261 \\
115 \\
165 \\
.470 \\
296 \\
599 \\
530 \\
342 \\
99 \\
55\end{array}$} \\
\hline & & & & & & & 3.1 & & 4.86 & 1,150 & & 3,520 \\
\hline
\end{tabular}




\section{CENTER DITCH BELOW KOLEA RESERVOIR, NEAR HUELO, MAUI}

LocATION.-Water-stage recorder 200 feet below intake from Kolea Reservoir spillway, half a mile below intake in Waikamoi Stream, and $1 \% \frac{1}{4}$ miles southeast of Kailua.

RecoRDs AVAIL ABLE.-March, 1918, to June, 1929.

Extremes.-Maximum discharge during year, 89 million gallons a day or 138 second-feet Feb. 16 (gage height, 5.39 feet); minimum, 0.9 million gallons a day or 1.4 second-feet June 30 .

1918-1929: Maximum discharge, 96 million gallons a day or 149 secondfeet Nov. 17, 1927 (gage height, 5.72 feet); ditch dry Dec. 4, 1918, and Jan. 16, 1919.

REMARKs. - Records good for ordinary stages; fair for extremely high stages, and poor for estimated periods. Center ditch receives the flow of Manuel Luis ditch and diverts water from all streams between Waikamoi and Kailua. Regulated by head gates and spillways.

Discharge, in million gallons a day, 1928-29

\begin{tabular}{|c|c|c|c|c|c|c|c|c|c|c|c|c|}
\hline Day & uly & ug. & Sept. & \begin{tabular}{l|l} 
Oct. & N
\end{tabular} & Nov. & Dec. & Jan. $\mathrm{F}$ & Feb. & Mar. & Apr. & May & June \\
\hline & $\begin{array}{l}47 \\
25 \\
21 \\
10.7 \\
5.0\end{array}$ & $\begin{array}{l}20 \\
25 \\
19.1 \\
25\end{array}$ & $\begin{array}{l}\mathbf{a} 2.9 \\
33 \\
31 \\
35 \\
20\end{array}$ & \begin{tabular}{r|r}
11.4 & \\
16.3 & 7.2 \\
5.1 & \\
15.8 &
\end{tabular} & \begin{tabular}{r|}
3.3 \\
2.4 \\
2.4 \\
6.9 \\
18.7
\end{tabular} & $\begin{array}{r}1.8 \\
1.6 \\
1.7 \\
1.5 \\
33\end{array}$ & 23 & $\begin{array}{l}6.2 \\
2.1 \\
2.0 \\
1.9 \\
1.7\end{array}$ & $\begin{array}{l}29 \\
29 \\
31 \\
45\end{array}$ & $\begin{array}{l}2.0 \\
7.6 \\
4.9 \\
1.8 \\
1.5\end{array}$ & $\begin{array}{l}2.8 \\
2.0 \\
1.8 \\
1.7 \\
1.6\end{array}$ & $\begin{array}{r}1.4 \\
1.2 \\
13.6 \\
19.4\end{array}$ \\
\hline $\begin{array}{l}8 \\
9 \\
9\end{array}$ & $\begin{array}{l}7.9 \\
24 \\
30 \\
17.3 \\
23\end{array}$ & $\begin{array}{r}18.2 \\
7.7 \\
4.4 \\
3.5 \\
16.7\end{array}$ & $\begin{array}{l}13.2 \\
23 \\
16.1 \\
27 \\
46\end{array}$ & \begin{tabular}{l|l}
4.1 & \\
3.0 & \\
3.0 & \\
2.5 & \\
2.5 &
\end{tabular} & $\begin{array}{l}2.4 \\
46 \\
20 \\
26 \\
40\end{array}$ & \begin{tabular}{l|l}
50 \\
28 \\
12.3 \\
38 \\
35
\end{tabular} & 18 & $\begin{array}{r}14.0 \\
12.6 \\
3.0 \\
2.3 \\
2.1\end{array}$ & $\begin{array}{l}28 \\
45 \\
36 \\
61 \\
38\end{array}$ & $\begin{array}{r}2.2 \\
2.9 \\
21.2 \\
2.2 \\
33\end{array}$ & $\begin{array}{l}9.9 \\
7.9 \\
6.8 \\
2.0 \\
2.4\end{array}$ & $\begin{array}{l}1.8 \\
1.4 \\
1.2 \\
1.2\end{array}$ \\
\hline 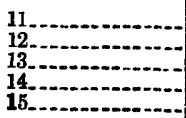 & $\begin{array}{r}22 \\
6.5 \\
9.2 \\
21.0 \\
17.4\end{array}$ & $\begin{aligned} 22 \\
17.5 \\
12.8 \\
13.6 \\
4.4\end{aligned}$ & $\begin{array}{l}36 \\
22 \\
11.8 \\
5.9 \\
12.1\end{array}$ & \begin{tabular}{l|l}
2.1 & \\
2.0 & \\
2.0 & \\
2.2 & \\
8.8 &
\end{tabular} & $\begin{array}{l}70 \\
27 \\
29 \\
33 \\
32\end{array}$ & 46 & 4.7 & $\begin{array}{l}5.0 \\
27 \\
4.1 \\
21 \\
37\end{array}$ & $\begin{array}{l}45 \\
29 \\
24 \\
28 \\
31\end{array}$ & $\begin{array}{l}63 \\
75 \\
35 \\
24 \\
24\end{array}$ & \begin{tabular}{l|}
18.8 \\
12.8 \\
34 \\
27 \\
14.9
\end{tabular} & $\begin{array}{r}2.3 \\
2.4 \\
6.4 \\
2.0 \\
24\end{array}$ \\
\hline - & $\begin{array}{l}35 \\
34 \\
27 \\
27 \\
14.8\end{array}$ & $\begin{array}{l}29 \\
36 \\
49 \\
25 \\
20\end{array}$ & $\begin{array}{r}4.8 \\
3.8 \\
4.8 \\
43 \\
10.5\end{array}$ & \begin{tabular}{l|l}
2.1 & \\
2.0 & \\
2.0 & \\
2.8 & \\
2.0 &
\end{tabular} & $\begin{array}{l}22 \\
14.9 \\
27 \\
21 \\
13.9\end{array}$ & 24 & . & $\begin{array}{l}70 \\
73 \\
50 \\
45 \\
61\end{array}$ & $\begin{array}{r}22 \\
16.6 \\
9.3 \\
5.7 \\
4.6\end{array}$ & $\begin{array}{r}8.4 \\
8.6 \\
35 \\
10.7 \\
4.2\end{array}$ & $\begin{array}{l}6.9 \\
5.3 \\
1.7 \\
1.6 \\
1.4\end{array}$ & $\begin{array}{l}3.3 \\
1.8 \\
1.7 \\
1.4 \\
1.4\end{array}$ \\
\hline - & $\begin{array}{l}5.3 \\
25 \\
24 \\
48 \\
59\end{array}$ & $\begin{array}{l}29 \\
18.0 \\
22 \\
31 \\
23\end{array}$ & $\begin{array}{l}7.7 \\
2.9 \\
2.8 \\
2.5 \\
2.3\end{array}$ & \begin{tabular}{l|l}
2.0 & 2 \\
2.4 & 1 \\
2.0 & 1 \\
1.8 & \\
1.7 &
\end{tabular} & $\left.\begin{array}{r|}27 \\
14.5 \\
10.8 \\
7.6 \\
4.6\end{array}\right\}$ & 5.5 & 40 & $\begin{array}{l}55 \\
45 \\
31 \\
26 \\
19.1\end{array}$ & $\begin{array}{l}4.0 \\
3.3 \\
\text {.3. } \\
2.9 \\
2.6\end{array}$ & $\begin{array}{l}3.2 \\
2.8 \\
2.8 \\
3.8 \\
4.7\end{array}$ & \begin{tabular}{r|}
1.4 \\
1.5 \\
4.1 \\
3.3 \\
1.8
\end{tabular} & $\begin{array}{l}1.3 \\
1.3 \\
1.3 \\
1.6 \\
1.6\end{array}$ \\
\hline - & $\begin{array}{l}66 \\
56 \\
29 \\
23 \\
20 \\
13.2\end{array}$ & $\begin{array}{r}10.2 \\
5.2 \\
14.0 \\
13.8 \\
3.5 \\
3.0\end{array}$ & $\begin{array}{r}2.2 \\
2.0 \\
2.2 \\
14.4 \\
2.1 \\
\end{array}$ & \begin{tabular}{c|c}
1.6 & \\
28 & \\
22 & \\
2.9 & \\
3.7 &. \\
2.9 &.
\end{tabular} & \begin{tabular}{r|}
3.1 \\
2.3 \\
2.0 \\
2.0 \\
2.5
\end{tabular} & 16 & \begin{tabular}{l|l}
7.8 & \\
3.5 & \\
2.8 & - \\
6.1 & -
\end{tabular} & $\begin{array}{l}20 \\
37 \\
46\end{array}$ & $\begin{array}{l}2.5 \\
2.3 \\
2.2 \\
2.0 \\
2.0 \\
2.0\end{array}$ & $\begin{array}{l}4.6 \\
4.0 \\
3.3 \\
3.3 \\
3.4 \\
--.--\end{array}$ & $\begin{array}{r}1.6 \\
10.5 \\
4.8 \\
2.0 \\
1.5 \\
1.3\end{array}$ & $\begin{array}{r}1.4 \\
1.1 \\
1.0 \\
1.0 \\
.9 \\
\hdashline-. .\end{array}$ \\
\hline \multirow{2}{*}{\multicolumn{4}{|c|}{ Month }} & \multicolumn{4}{|c|}{ Million gallons a day } & \multirow{2}{*}{\multicolumn{2}{|c|}{$\begin{array}{c}\text { Second- } \\
\text { feet } \\
\text { (mean) }\end{array}$}} & \multicolumn{3}{|c|}{ Total run-off } \\
\hline & & & & Maximum & Min & imum & Mean & & & $\begin{array}{l}\text { Million } \\
\text { gallons }\end{array}$ & \multicolumn{2}{|c|}{ Acre-feet } \\
\hline \multicolumn{4}{|c|}{ 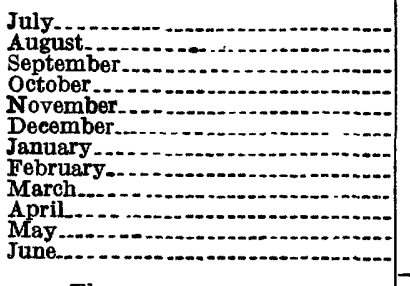 } & $\begin{array}{l}66 \\
49 \\
46 \\
28 \\
70 \\
59 \\
40 \\
73 \\
61 \\
75 \\
34 \\
24\end{array}$ & & $\begin{array}{l}5.0 \\
3.0 \\
2.0 \\
1.6 \\
2.0 \\
1.5 \\
2.8 \\
1.7 \\
2.0 \\
1.5 \\
1.3 \\
.0\end{array}$ & $\begin{array}{c}25.6 \\
18.4 \\
14.8 \\
5.48 \\
17.8 \\
22.5 \\
14.3 \\
22.7 \\
20.0 \\
13.4 \\
6.36 \\
3.55\end{array}$ & \multicolumn{2}{|c|}{\begin{tabular}{l|}
39.6 \\
28.5 \\
22.9 \\
8.48 \\
27.5 \\
34.8 \\
22.1 \\
39.8 \\
30.9 \\
20.7 \\
9.84 \\
5.49
\end{tabular}} & $\begin{array}{l}720 \\
619 \\
403 \\
197 \\
106\end{array}$ & \multicolumn{2}{|r|}{$\begin{array}{r}2,440 \\
1,750 \\
1,360 \\
521 \\
1,640 \\
2,140 \\
1,360 \\
2,210 \\
1,900 \\
1,230 \\
605 \\
327\end{array}$} \\
\hline \multicolumn{4}{|c|}{ 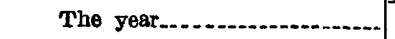 } & 75 & & .9 & 15.6 & & 24.1 & 6,700 & & 17,500 \\
\hline
\end{tabular}

- Partly estimated. 


\section{NAIIIIIHAELE STREAM IEAR HUELO, MAUI}

Location.-Water-stage recorder 200 feet above Wailoa ditch intake, 700 feet above New Hamakua ditch trail, and 11/2 miles south of Kailua.

Drain Age are A. - 2.8 square miles.

Records avamable.-October, 1913, to June, 1918; August, 1919, to June, 1929.

EXTremes.-Maximum discharge, 365 million gallons a day or 565 second-feet Oct. 27 (gage height, 5.00 feet); minimum, 3.8 million gallons a day or $\mathbf{5 . 9}$ second-feet Oct. 26.

1913-1929: Maximum discharge, 1,800 million gallons a day or 2,790 second-feet May, 1916 (gage height, 6.3 feet); minimum, 0.45 million gallons a day or 0.7 second-foot July 14, 1920.

REMARKs.-Records good for ordinary stages, fair for estimated periods, and poor for high stages. No diversions.

Discharge, in million gallons a day, 1928-29

\begin{tabular}{|c|c|c|c|c|c|c|c|c|c|c|c|c|}
\hline a & July & Aug. & Sept. & Oct. & Nov. & Dec. & Jan. & Feb. & Mar. & Apr. & May & June \\
\hline $\begin{array}{l}1 \ldots \\
2 \ldots \\
3 \ldots- \\
5 \ldots\end{array}$ & $\begin{array}{l}54 \\
25 \\
19.2 \\
15.3 \\
12.4\end{array}$ & $\begin{array}{l}39 \\
19.0 \\
31 \\
23 \\
630\end{array}$ & $\begin{array}{l}9.5 \\
65 \\
37 \\
32 \\
17.3\end{array}$ & $\begin{array}{r}13.3 \\
12.2 \\
7.2 \\
8.8 \\
18.6\end{array}$ & $\begin{array}{r}9.7 \\
7.6 \\
6.1 \\
17.4 \\
21\end{array}$ & 6.5 & $\begin{array}{l}38 \\
14.2 \\
35 \\
20 \\
15.8\end{array}$ & $\begin{array}{l}7.8 \\
7.2 \\
6.8 \\
6.4 \\
5.9\end{array}$ & 50 & $\begin{array}{l}5.4 \\
6.4 \\
9.0 \\
8.0 \\
\text { 5. } 5\end{array}$ & $\begin{array}{l}7.8 \\
5.8 \\
5.0 \\
4.7 \\
5.4\end{array}$ & $\begin{array}{r}5.9 \\
5.8 \\
5.4 \\
11.1 \\
25\end{array}$ \\
\hline $\begin{array}{r}6 \ldots \\
7 \ldots \\
8 \\
9 \\
10 \ldots\end{array}$ & $\begin{array}{l}10.8 \\
17.8 \\
43 \\
18.9 \\
35\end{array}$ & $\begin{array}{l}16.1 \\
15.8 \\
13.4 \\
11.5 \\
29\end{array}$ & $\begin{array}{l}13.7 \\
30 \\
15.3 \\
32 \\
87\end{array}$ & $\begin{array}{l}9.8 \\
8.2 \\
7.0 \\
5.9 \\
5.5\end{array}$ & $\begin{array}{l}8.2 \\
88 \\
26 \\
24 \\
36\end{array}$ & $\begin{array}{l}60 \\
\\
25 \\
45 \\
50\end{array}$ & $\begin{array}{l}40 \\
20 \\
58 \\
19.6 \\
14.8\end{array}$ & \begin{tabular}{l|}
23 \\
30 \\
10.6 \\
7.2 \\
6.4
\end{tabular} & 70 & $\begin{array}{c}7.0 \\
10.7 \\
38 \\
9.5 \\
73\end{array}$ & $\begin{array}{l}36 \\
24 \\
18.1 \\
8.6 \\
15.0\end{array}$ & $\begin{array}{r}12.2 \\
7.8 \\
6.1 \\
5.4 \\
\mathbf{5 . 5}\end{array}$ \\
\hline (n) & $\begin{array}{l}28 \\
17.3 \\
17.3 \\
19.2 \\
14.8\end{array}$ & $\begin{array}{l}25 \\
27 \\
25 \\
20 \\
17.6\end{array}$ & $\begin{array}{l}46 \\
20 \\
15.6 \\
13.2 \\
11.7\end{array}$ & $\begin{array}{r}5.0 \\
4.8 \\
4.6 \\
8.2 \\
13.8\end{array}$ & 61 & $\begin{array}{r}47 \\
58 \\
85 \\
101 \\
48\end{array}$ & $\begin{array}{l}12.7 \\
10.8 \\
10.3 \\
50 \\
24\end{array}$ & $\begin{array}{l}8.0 \\
52 \\
13.1 \\
36 \\
92\end{array}$ & 28 & $\begin{array}{r}113 \\
120 \\
42 \\
26 \\
24\end{array}$ & $\begin{array}{l}36 \\
23 \\
43 \\
48 \\
23\end{array}$ & $\begin{array}{l}11.8 \\
9.9 \\
15.1 \\
10.4 \\
43\end{array}$ \\
\hline 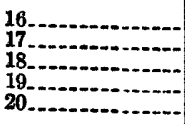 & $\begin{array}{l}69 \\
50 \\
30 \\
34 \\
22\end{array}$ & $\begin{array}{l}64 \\
99 \\
45 \\
23 \\
30\end{array}$ & $\begin{array}{r}13.2 \\
9.9 \\
11.3 \\
87 . \\
15.8\end{array}$ & $\begin{array}{l}7.0 \\
5.5 \\
5.2 \\
5.0 \\
4.6\end{array}$ & 34 & $\begin{array}{l}57 \\
25 \\
38 \\
22 \\
18.9\end{array}$ & $\begin{array}{l}14.5 \\
11.0 \\
11.2 \\
16.7 \\
12.0\end{array}$ & $\begin{array}{r}122 \\
148 \\
90 \\
58 \\
110\end{array}$ & $\begin{array}{l}23 \\
17.0 \\
14.5 \\
14.8 \\
13.5\end{array}$ & $\begin{array}{l}16.1 \\
19.4 \\
65 \\
21 \\
14.8\end{array}$ & $\begin{array}{r}14.5 \\
12.0 \\
9.9 \\
8.8 \\
8.0\end{array}$ & $\begin{array}{r}14.7 \\
9.7 \\
8.4 \\
7.2 \\
6.6\end{array}$ \\
\hline $23 \ldots$ & $\begin{array}{l}17.0 \\
44 \\
26 \\
73 \\
90\end{array}$ & $\begin{array}{l}21 \\
21 \\
21 \\
32 \\
16.4\end{array}$ & $\begin{array}{r}11.7 \\
10.1 \\
9.0 \\
8.2 \\
7.6\end{array}$ & $\begin{array}{l}5.2 \\
9.9 \\
5.2 \\
4.6 \\
4.2\end{array}$ & 22 & $\begin{array}{l}20 \\
16.7 \\
13.7 \\
22 \\
76\end{array}$ & $\begin{array}{c}19.8 \\
136 \\
46 \\
20 \\
15.0\end{array}$ & $\begin{array}{l}63 \\
92 \\
38 \\
42 \\
21\end{array}$ & $\begin{array}{r}12.4 \\
9.2 \\
8.4 \\
7.8 \\
7.2\end{array}$ & $\begin{array}{l}12.4 \\
11.2 \\
10.3 \\
11.0 \\
10.8\end{array}$ & $\begin{array}{r}7.2 \\
7.4 \\
18.4 \\
13.0 \\
8.0\end{array}$ & $\begin{array}{l}6.1 \\
5.7 \\
5.2 \\
5.9 \\
6.3\end{array}$ \\
\hline 31 & $\begin{array}{l}122 \\
73 \\
30 \\
23 \\
18.6 \\
17.0\end{array}$ & $\begin{array}{r}14.5 \\
13.8 \\
22.5 \\
11.5 \\
10.1 \\
9.5\end{array}$ & $\begin{array}{r}6.8 \\
6.4 \\
8.2 \\
14.2 \\
7.2 \\
\hdashline\end{array}$ & $\begin{array}{c}3.8 \\
7.5 \\
23 \\
9.0 \\
7.2 \\
6.4\end{array}$ & 11 & $\begin{array}{l}40 \\
19.2 \\
28 \\
34 \\
16.1 \\
13.9\end{array}$ & $\begin{array}{l}12.9 \\
11.0 \\
23 \\
12.2 \\
\cdot 9.7 \\
8.6\end{array}$ & 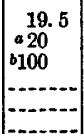 & $\begin{array}{l}\text { 6. } 8 \\
6.3 \\
6.1 \\
5.5 \\
5.2 \\
5.0\end{array}$ & $\begin{array}{l}8.0 \\
7.8 \\
6.7 \\
7.0 \\
7.0 \\
-. .\end{array}$ & $\begin{array}{r}6.8 \\
11.6 \\
28.6 \\
11.0 \\
7.2 \\
6.6\end{array}$ & $\begin{array}{l}6.1 \\
4.6 \\
4.2 \\
4.1 \\
4.4\end{array}$ \\
\hline \multirow{2}{*}{\multicolumn{4}{|c|}{ Month }} & \multicolumn{4}{|c|}{ Million gallons a day } & \multirow{2}{*}{\multicolumn{2}{|c|}{$\begin{array}{l}\text { Second- } \\
\text { feet } \\
\text { (mean) }\end{array}$}} & \multicolumn{3}{|c|}{ Total run-off } \\
\hline & & & & Maximum & Min & mum & Mean & & & $\begin{array}{l}\text { Million } \\
\text { gallons }\end{array}$ & Acr & $\theta-f e \theta t$ \\
\hline $\begin{array}{l}\text { July } \\
\text { August } \\
\text { September } \\
\text { October } \\
\text { November. } \\
\text { December.. } \\
\text { January } \\
\text { February } \\
\text { March } \\
\text { April } \\
\text { May. } \\
\text { June. }\end{array}$ & 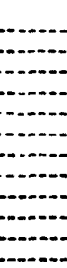 & & $\ldots$ & $\begin{array}{r}122 \\
99 \\
87 \\
75 \\
-101 \\
136 \\
148 \\
-120 \\
48 \\
43\end{array}$ & & \begin{tabular}{r}
10.8 \\
9.5 \\
6.4 \\
3.8 \\
\hdashline \\
8.6 \\
5.9 \\
5.0 \\
5.4 \\
4.7 \\
4.1
\end{tabular} & $\begin{array}{r}35 . \\
25.7 \\
22 . \\
10 . \\
25 . \\
36 . \\
24 . \\
44 . \\
31 . \\
24 . \\
15 . \\
9 .\end{array}$ & & $\begin{array}{l}54.3 \\
39.8 \\
34.7 \\
15.6 \\
38.8 \\
56.2 \\
38.1 \\
68.2 \\
48.1 \\
37.4 \\
24.0 \\
14.4\end{array}$ & $\begin{array}{r}1,090 \\
796 \\
672 \\
314 \\
754 \\
1,130 \\
763 \\
1,240 \\
965 \\
726 \\
482 \\
279\end{array}$ & & $\begin{array}{r}3,340 \\
2,440 \\
2,060 \\
961 \\
2,310 \\
3,450 \\
2,340 \\
3,790 \\
2,960 \\
2,230 \\
1,470 \\
857\end{array}$ \\
\hline The.year & $\ldots$ & . & & 148 & & 3.8 & 25.2 & & 39.0 & 9,210 & & 28,200 \\
\hline
\end{tabular}

- Partly estimated.

- Estimated. 


\section{KAIYUA STREAM NEAR HUELO, MAUI}

Location.-Water-stage recorder just above Wailoa ditch intake, $1 \frac{1}{4}$ miles southwest of Kailua, and $2 \frac{1}{2}$ miles south of Huelo.

Drainage area.-3.0 square miles.

Records Avarlable. - December, 1910, to June, 1918; July, 1919, to June, 1929.

Extrames.-Maximum discharge during year, 915 million gallons a day or 1,420 second-feet $\mathrm{Feb} .17$ (gage height, 8.10 feet); minimum, 2.0 million gallons a day or 3.1 second-feet Oct. 26 and 27.

1911-1918, 1919-1929: Maximum discharge, about 1,500 million gallons a day or 2,300 second-feet Feb. 1, 1922 (gage height, 10.5 feet; determined from floodmarks); minimum, 0.07 million gallons a day or 0.11 second-feet June 27, 1921.

REMARKs.- Records for ordinary stages fair; for high and low stages poor. Partly estimated Sept. 7, 8, 14-18, 21-30, Oct. 1-15. No diversions above station.

Discharge, in million gallons a day, 1928-29

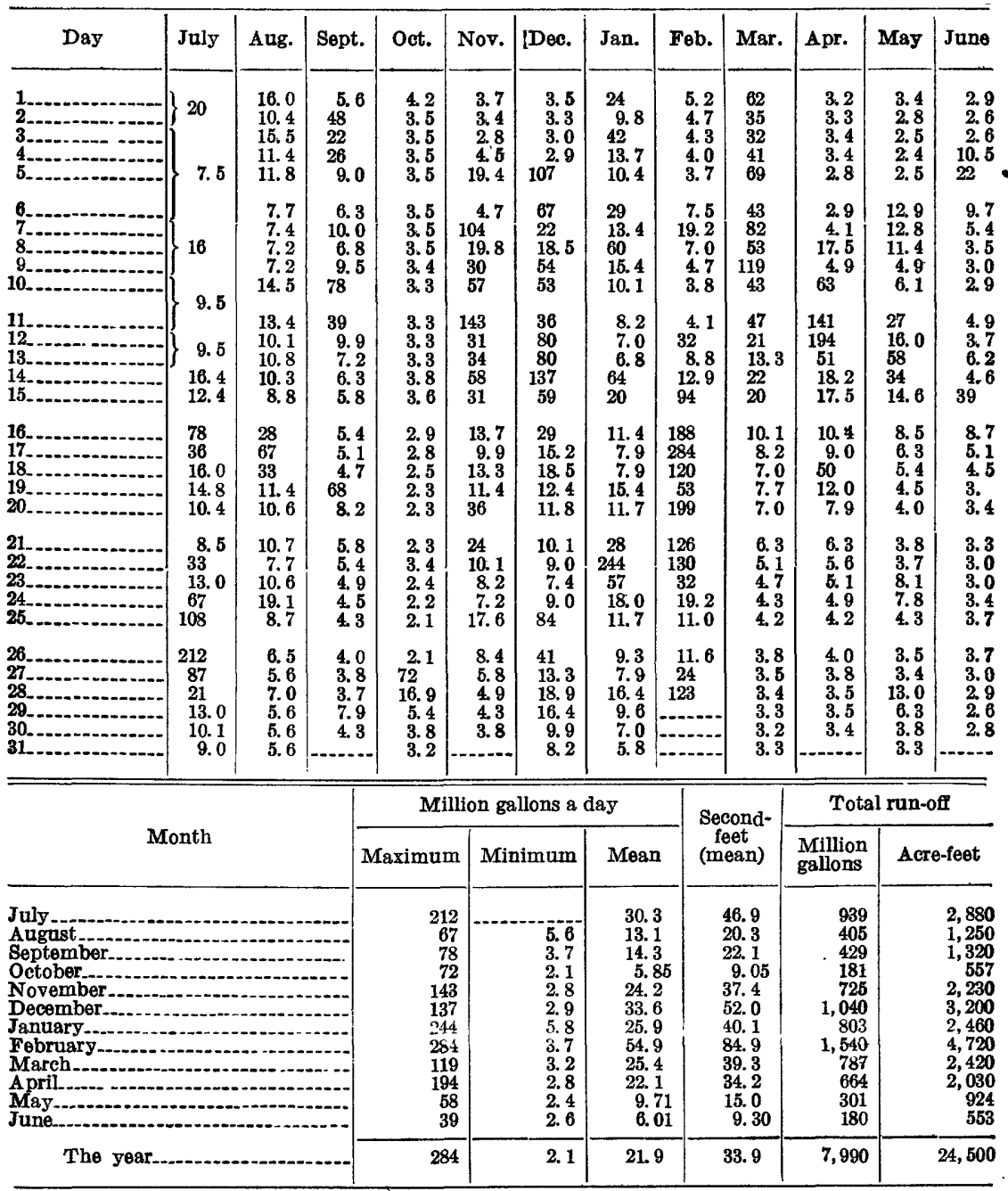




\section{HOOLAWAIIIII STREAM IEAR HUELO, MAUI}

Location.-Water-stage recorder just above New Hamakua ditch crossing, 2 miles west of Kailua, and 2 miles southwest of Huelo.

Records AVAILABLE.-April, 1911, to June, 1929.

EXTREMEs.-Maximum discharge during year, 220 million gallons a day or 340 second-feet Dec. 13 (gage height, 3.83 feet); minimum, 1.7 million gallons a day or 2.6 second-feet Oct. 13 .

1911-1929: Maximum discharge, 485 million gallons a day or 750 secondfeet Nov. 21, 1921 (gage height, 4.82 feet); minimum, 0.2 million gallons a day or 0.3 second-foot June 8,1926 .

REMARKs.- Records good for medium stages, poor for high stages, and fair where estimated. No diversions.

Discharge, in million gallons a day, 1928-29

\begin{tabular}{|c|c|c|c|c|c|c|c|c|c|c|c|c|c|}
\hline Day & July & Aug. & Sept. & Oct. & Nov. & Dec. & Jan. & \multicolumn{2}{|c|}{ Feb. } & Mar. & Apr. & May & June \\
\hline & $\begin{array}{r}9.5 \\
3.8\end{array}$ & $\begin{array}{l}5.1 \\
4.4 \\
5.6 \\
4.2 \\
5.8\end{array}$ & $\begin{array}{l}2.7 \\
8.5 \\
5.1 \\
4.4 \\
3.6\end{array}$ & & $\begin{array}{l}2.3 \\
2.1 \\
2.0 \\
4.8 \\
3.2\end{array}$ & $\begin{array}{r}2.5 \\
2.4 \\
2.3 \\
2.3 \\
21\end{array}$ & $\begin{array}{l}5.3 \\
3.8 \\
3.6 \\
3.4 \\
3.2\end{array}$ & \multicolumn{2}{|c|}{$\begin{array}{l}2.8 \\
2.7 \\
2.7 \\
2.5 \\
2.4\end{array}$} & $\begin{array}{r}13.7 \\
8.9 \\
8.9 \\
8.6 \\
14.7\end{array}$ & $\begin{array}{l}2.3 \\
2.1 \\
2.1 \\
2.1 \\
2.0\end{array}$ & $\begin{array}{l}2.4 \\
2.3 \\
2.3 \\
2.3 \\
2.3\end{array}$ & $\begin{array}{l}2.6 \\
2.7 \\
2.6 \\
2.7 \\
2.8\end{array}$ \\
\hline $\begin{array}{r}6 \\
7 \\
8 \\
8 \\
9 \\
10 . . .\end{array}$ & $\begin{array}{l}3.0 \\
2.8 \\
3.8 \\
4.0 \\
4.4\end{array}$ & $\begin{array}{l}3.8 \\
3.4 \\
3.0 \\
2.7 \\
\text { 3. }\end{array}$ & $\begin{array}{r}3.4 \\
6.2 \\
3.8 \\
5.3 \\
21\end{array}$ & 2.0 & $\begin{array}{r}2.3 \\
11.3 \\
4.7 \\
3.8 \\
3.4\end{array}$ & $\begin{array}{l}8.0 \\
4.8 \\
4.6 \\
7.0 \\
9.5\end{array}$ & $\begin{array}{r}3.8 \\
3.6 \\
17.2 \\
4.6 \\
3.8\end{array}$ & \multicolumn{2}{|c|}{$\begin{array}{l}3.9 \\
4.2 \\
3.0 \\
2.7 \\
2.5\end{array}$} & $\begin{array}{r}10.7 \\
10.1 \\
8.6 \\
43 \\
14.6\end{array}$ & $\begin{array}{r}2.0 \\
1.9 \\
2.5 \\
2.0 \\
16.3\end{array}$ & $\begin{array}{l}3.8 \\
2.7 \\
2.7 \\
2.7 \\
3.0\end{array}$ & $\begin{array}{l}2.7 \\
2.4 \\
.2 .4 \\
2.3 \\
2.3\end{array}$ \\
\hline $\begin{array}{l}13 \ldots \\
14 \ldots \\
15\end{array}$ & $\begin{array}{l}5.1 \\
3.8 \\
3.4 \\
3.2 \\
3.2\end{array}$ & $\begin{array}{l}3.8 \\
5.0 \\
4.2 \\
4.2 \\
3.2\end{array}$ & 10 & $\begin{array}{l}1.8 \\
1.8 \\
2.0\end{array}$ & $\begin{array}{l}6.4 \\
3.8 \\
5.4 \\
9.6 \\
7.3\end{array}$ & $\begin{array}{l}8.0 \\
11.1 \\
25 \\
24 \\
12.3\end{array}$ & $\begin{array}{l}\text { 3. } 4 \\
3.0 \\
2.8 \\
5.3 \\
6.0\end{array}$ & \multicolumn{2}{|c|}{$\begin{array}{r}\mathbf{3 .} 0 \\
8.3 \\
\mathbf{3 .} 4 \\
\mathbf{5 . 4} \\
\mathbf{1 8 . 6}\end{array}$} & $\begin{array}{r}21 \\
10.1 \\
7.2 \\
11.1 \\
8.8\end{array}$ & $\begin{array}{r}13.0 \\
11.4 \\
6.9 \\
5.1 \\
4.4\end{array}$ & $\begin{array}{l}3.2 \\
3.0 \\
4.0 \\
4.4 \\
4.2\end{array}$ & $\begin{array}{l}2.4 \\
2.1 \\
2.4 \\
2.4 \\
4.5\end{array}$ \\
\hline $\begin{array}{l}16 \\
17 \\
18 \\
19\end{array}$ & $\begin{array}{r}13.4 \\
6.9 \\
4.6 \\
4.4 \\
4.2\end{array}$ & $\begin{array}{r}8.1 \\
14.9 \\
10.8 \\
5.6 \\
4.4\end{array}$ & 20 & $\begin{array}{l}1.9 \\
1.9 \\
1.9 \\
1.9 \\
1.9\end{array}$ & $\begin{array}{l}5.1 \\
4.4 \\
5.1 \\
4.2 \\
4.0\end{array}$ & $\begin{array}{r}13.1 \\
7.7 \\
8.3 \\
6.4 \\
5.1\end{array}$ & $\begin{array}{l}\text { 3. } 6 \\
\text { 3. } \\
\text { 3. } \\
\text { 3. } 6 \\
\text { 3. } 2\end{array}$ & \multicolumn{2}{|c|}{$\begin{array}{l}38 \\
47 \\
29 \\
22 \\
24\end{array}$} & $\begin{array}{l}5.8 \\
4.8 \\
4.4 \\
4.6 \\
4.2\end{array}$ & $\begin{array}{r}3.8 \\
3.8 \\
12.7 \\
5.1 \\
4.0\end{array}$ & $\begin{array}{l}3.8 \\
3.4 \\
3.2 \\
3.2 \\
3.0\end{array}$ & $\begin{array}{l}2.7 \\
2.3 \\
2.4 \\
2.3 \\
2.3\end{array}$ \\
\hline 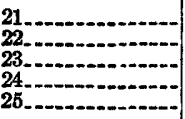 & \begin{tabular}{r|}
3.8 \\
4.2 \\
4.6 \\
9.5 \\
14.4
\end{tabular} & $\begin{array}{l}3.8 \\
3.6 \\
3.2 \\
3.9 \\
3.6\end{array}$ & 4.2 & $\begin{array}{l}1.8 \\
2.0 \\
1.9 \\
1.9 \\
1.8\end{array}$ & $\begin{array}{l}3.8 \\
3.4 \\
3.2 \\
3.0 \\
\text { 5. } 1\end{array}$ & $\begin{array}{r}5.1 \\
4.6 \\
4.0 \\
4.6 \\
13.4\end{array}$ & $\begin{array}{r}4.9 \\
31 \\
10.1 \\
5.8 \\
4.8\end{array}$ & \multicolumn{2}{|c|}{$\begin{array}{r}12.9 \\
18.4 \\
10.1 \\
9.0 \\
\text { 6. }\end{array}$} & $\begin{array}{l}3.8 \\
3.2 \\
3.0 \\
2.8 \\
2.7\end{array}$ & $\begin{array}{l}3.6 \\
3.4 \\
2.8 \\
2.8 \\
2.5\end{array}$ & $\begin{array}{l}\text { 3. } 2 \\
\text {.2 } \\
\text { 3. } \\
\text { 3. } 4 \\
\text { 3. } 0\end{array}$ & $\begin{array}{l}2.3 \\
2.1 \\
2.1 \\
2.1 \\
2.1\end{array}$ \\
\hline \multirow[t]{3}{*}{$\begin{array}{l}26 \\
28 \\
28 \\
30 \\
31\end{array}$} & \begin{tabular}{r|}
23 \\
16.5 \\
7.5 \\
5.6 \\
4.4 \\
3.8
\end{tabular} & $\begin{array}{l}3.4 \\
3.4 \\
4.1 \\
3.2 \\
3.0 \\
2.7\end{array}$ & 2.6 & \begin{tabular}{r|r}
1.7 & \\
10.6 & \\
4.0 & \\
2.4 & \\
2.3 & \\
2.1 &
\end{tabular} & $\begin{array}{r}3.2 \\
2.8 \\
2.8 \\
2.7 \\
2.6 \\
\end{array}$ & $\begin{array}{l}8.1 \\
4.8 \\
5.7 \\
6.7 \\
4.4 \\
3.8\end{array}$ & $\begin{array}{r}\text { 3. } 8 \\
3.6 \\
\text { 5. } \\
\text { 3. } 6 \\
\text { 3.2 } \\
\text { 3. } 0\end{array}$ & \multicolumn{2}{|c|}{$\begin{array}{r}5.6 \\
5.0 \\
19.5 \\
\hdashline \\
\hdashline \\
\hdashline\end{array}$} & $\begin{array}{l}2.5 \\
2.5 \\
2.4 \\
2.4 \\
2.3 \\
2.3\end{array}$ & $\begin{array}{l}2.4 \\
2.3 \\
2.3 \\
2.1 \\
2.3 \\
\end{array}$ & $\begin{array}{l}3.0 \\
3.4 \\
4.3 \\
3.2 \\
2.8 \\
2.7\end{array}$ & $\begin{array}{l}2.1 \\
2.1 \\
2.1 \\
2.0 \\
2.0 \\
\end{array}$ \\
\hline & \multirow[b]{2}{*}{ Month } & & \multirow[b]{2}{*}{ - } & \multicolumn{4}{|c|}{ Million gallons a day } & \multirow{2}{*}{\multicolumn{3}{|c|}{$\begin{array}{l}\text { Second- } \\
\text { feet } \\
\text { (mean) }\end{array}$}} & \multicolumn{3}{|c|}{ Total run-off } \\
\hline & & & & Maximum & Min & mum & Mear & & & & $\begin{array}{l}\text { Million } \\
\text { gallons }\end{array}$ & \multicolumn{2}{|c|}{ Acre-feet } \\
\hline $\begin{array}{l}\text { July } \\
\text { August } \\
\text { September } \\
\text { October-.. } \\
\text { November } \\
\text { December-.. } \\
\text { January } \\
\text { Pebruary } \\
\text { March } \\
\text { April } \\
\text { May } \\
\text { June }\end{array}$ & & & & $\begin{array}{l}23 \\
14.9 \\
10.6 \\
11.3 \\
25 \\
31 \\
47 \\
43 \\
16.3 \\
4.4 \\
4.5\end{array}$ & & \begin{tabular}{l}
2.8 \\
2.7 \\
\hdashline 2.0 \\
2.3 \\
2.8 \\
2.4 \\
2.3 \\
1.9 \\
2.3 \\
2.0
\end{tabular} & \multicolumn{2}{|c|}{$\begin{array}{r}6.20 \\
4.62 \\
5.40 \\
2.31 \\
4.26 \\
8.08 \\
5.44 \\
11.2 \\
8.18 \\
4.40 \\
3.15 \\
2.40\end{array}$} & \multicolumn{2}{|c|}{$\begin{array}{c}9.59 \\
7.15 \\
8.36 \\
3.57 \\
6.59 \\
12.5 \\
8.42 \\
17.3 \\
12.7 \\
6.81 \\
4.87 \\
3.71\end{array}$} & $\begin{array}{c}192 \\
143 \\
162 \\
71.6 \\
128 \\
251 \\
169 \\
314 \\
254 \\
132 \\
97.7 \\
72.1\end{array}$ & \multicolumn{2}{|r|}{$\begin{array}{l}590 \\
440 \\
497 \\
220 \\
392 \\
769 \\
518 \\
962 \\
778 \\
405 \\
300 \\
221\end{array}$} \\
\hline \multicolumn{3}{|l|}{ The year... } & & 47 & & 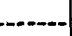 & 5.4 & 44 & & 8.42 & 1,990 & \multicolumn{2}{|r|}{6,090} \\
\hline
\end{tabular}

- Estimated. 


\section{HOOLAW ANUI STREAM NEAR HUELO, MAUI}

Location.- Water-stage recorder just above intake of Wailoa ditch, 2 miles west of Kailua, and 2 miles southwest of Huelo; elevation 1,240 feet.

RecoRds avaILABLE.-December, 1910, to June, 1929.

Extremes.-Maximum discharge during year, 156 million gallons a day or 241 second-feet Feb. 17 (gage height, 4.10 feet); minimum uncertain, probably occurred between June 26 and 30 .

1910-1929: Maximum discharge, about 550 million gallons a day or 851 second-feet Feb 1, 1922 (gage height, 8.40 feet); minimum, 0.15 million gallons a day or 0.2 second-foot Oct. $25,1917$.

REMARKs. - Records good for ordinary stages, fair for estimated periods and high stages. No diversions.

Discharge, in million gallons a day, 1928-29

\begin{tabular}{|c|c|c|c|c|c|c|c|c|c|c|c|c|}
\hline Day & July & Aug. & Sept. & Oct. & Nov. & Dec. & Jan. & Feb. & Mar. & Apr. & May & June \\
\hline $\begin{array}{l}1 \ldots \\
2 \ldots \\
3-\ldots \\
4-. \\
5 \ldots\end{array}$ & $\begin{array}{r}16.7 \\
5.8 \\
5.1 \\
4.4 \\
3.8\end{array}$ & $\begin{array}{l}9.0 \\
6.8 \\
9.1 \\
6.9 \\
8.2\end{array}$ & $\begin{array}{r}3.2 \\
18.2 \\
8.5 \\
8.0 \\
5.2\end{array}$ & $\begin{array}{l}2.9 \\
2.6 \\
2.2 \\
2.2 \\
3.0\end{array}$ & $\begin{array}{l}2.3 \\
2.0 \\
1.6 \\
3.6 \\
8.4\end{array}$ & $\begin{array}{r}3.0 \\
2.9 \\
2.7 \\
2.5 \\
38\end{array}$ & $\begin{array}{r}10.4 \\
5.6 \\
8.7 \\
6.3 \\
5.6\end{array}$ & $\begin{array}{l}3.9 \\
3.6 \\
3.4 \\
3.1 \\
2.8\end{array}$ & $\begin{array}{l}32 \\
20 \\
20 \\
21 \\
31\end{array}$ & $\begin{array}{r}1.9 \\
1.9 \\
1.9 \\
1.9 \\
1.6\end{array}$ & $\begin{array}{l}2.3 \\
1.9 \\
1.8 \\
1.8 \\
1.7\end{array}$ & $\begin{array}{l}1.3 \\
1.2 \\
1.2 \\
1.6 \\
2.7\end{array}$ \\
\hline $\begin{array}{r}6 \\
7 \\
8 \\
9 \\
9 \\
10\end{array}$ & $\begin{array}{l}3.6 \\
4.1 \\
7.6 \\
4.7 \\
9.0\end{array}$ & $\begin{array}{l}5.5 \\
5.3 \\
4.7 \\
4.2 \\
6.3\end{array}$ & $\begin{array}{r}4.4 \\
7.8 \\
4.4 \\
6.1 \\
35\end{array}$ & $\begin{array}{l}2.3 \\
2.0 \\
2.0 \\
1.8 \\
1.7\end{array}$ & \begin{tabular}{c|}
2.4 \\
32 \\
7.9 \\
6.3 \\
8.5
\end{tabular} & $\begin{array}{r}19.1 \\
8.7 \\
9.0 \\
17.9 \\
22\end{array}$ & \begin{tabular}{r|}
10.4 \\
6.9 \\
30 \\
9.0 \\
6.5
\end{tabular} & $\begin{array}{l}4.7 \\
7.1 \\
3.8 \\
2.9 \\
2.4\end{array}$ & $\begin{array}{l}24 \\
30 \\
22 \\
61 \\
28\end{array}$ & $\begin{array}{r}1.6 \\
1.7 \\
4.2 \\
2.3 \\
21\end{array}$ & $\begin{array}{l}4.2 \\
3.0 \\
3.3 \\
2.3 \\
2.5\end{array}$ & $\begin{array}{l}2.0 \\
1.4 \\
1.2 \\
1.0 \\
1.0\end{array}$ \\
\hline 13 & $\begin{array}{l}6.2 \\
4.9 \\
4.8 \\
5.5 \\
5.1\end{array}$ & $\begin{array}{l}5.8 \\
6.4 \\
6.0 \\
5.7 \\
4.5\end{array}$ & $\begin{array}{l}21 \\
8.6 \\
6.5 \\
5.5 \\
4.9\end{array}$ & $\begin{array}{l}1.6 \\
1.6 \\
1.6 \\
1.8 \\
2.3\end{array}$ & \begin{tabular}{l|}
29 \\
12.4 \\
15.8 \\
27 \\
19.4
\end{tabular} & $\begin{array}{l}20 \\
33 \\
43 \\
57 \\
36\end{array}$ & $\begin{array}{r}5.5 \\
4.7 \\
4.3 \\
15.4 \\
9.2\end{array}$ & $\begin{array}{r}2.5 \\
14.8 \\
5.4 \\
6.0 \\
42\end{array}$ & $\begin{array}{l}32 \\
16.5 \\
12.0 \\
15.9 \\
13.0\end{array}$ & $\begin{array}{l}37 \\
46 \\
19.0 \\
10.4 \\
8.0\end{array}$ & $\begin{array}{l}6.8 \\
4.3 \\
7.9 \\
9.1 \\
6.4\end{array}$ & $\begin{array}{l}1.6 \\
1.4 \\
1.9 \\
1.5 \\
7.4\end{array}$ \\
\hline $\begin{array}{l}16 \\
17 \\
18 \\
20\end{array}$ & $\begin{array}{l}30 \\
16.5 \\
10.8 \\
9.7 \\
7.2\end{array}$ & $\begin{array}{r}14.3 \\
33 \\
19.5 \\
9.2 \\
7.9\end{array}$ & $\begin{array}{r}4.7 \\
4.0 \\
4.0 \\
33 \\
6.4\end{array}$ & $\begin{array}{l}1.6 \\
1.6 \\
1.6 \\
1.4 \\
1.3\end{array}$ & $\begin{array}{r}10.0 \\
7.2 \\
7.9 \\
6.4 \\
9.3\end{array}$ & $\begin{aligned} 24 \\
13.8 \\
15.2 \\
10.2 \\
8.4\end{aligned}$ & $\begin{array}{l}6.3 \\
4.9 \\
4.8 \\
6.2 \\
5.3\end{array}$ & $\begin{array}{l}61 \\
93 \\
61 \\
33 \\
60\end{array}$ & $\begin{array}{l}8.0 \\
6.4 \\
5.6 \\
5.8 \\
5.2\end{array}$ & $\begin{array}{c}5.8 \\
5.6 \\
23 \\
7.4 \\
\mathbf{5 . 5}\end{array}$ & $\begin{array}{l}4.2 \\
3.5 \\
3.1 \\
2.8 \\
2.5\end{array}$ & $\begin{array}{l}2.8 \\
1.9 \\
1.7 \\
1.6 \\
1.4\end{array}$ \\
\hline $\begin{array}{l}21 \\
22 \\
24 \\
25\end{array}$ & $\begin{array}{r}6.0 \\
12.5 \\
6.7 \\
23 \\
42\end{array}$ & $\begin{array}{l}6.6 \\
5.7 \\
5.9 \\
6.7 \\
5.0\end{array}$ & $\begin{array}{l}5.2 \\
4.4 \\
4.0 \\
\text { 3. } \\
\text { 3. } 4\end{array}$ & $\begin{array}{l}1.4 \\
1.8 \\
1.3 \\
1.2 \\
1.2\end{array}$ & $\begin{array}{l}9.8 \\
5.6 \\
4.7 \\
4.6 \\
8.4\end{array}$ & $\begin{array}{r}7.9 \\
6.7 \\
5.8 \\
6.5 \\
30\end{array}$ & $\begin{array}{l}8.9 \\
75 \\
37 \\
16.0 \\
9.8\end{array}$ & $\begin{array}{l}37 \\
52 \\
28 \\
17.8 \\
11.4\end{array}$ & $\begin{array}{l}4.8 \\
3.8 \\
3.4 \\
3.0 \\
2.8\end{array}$ & $\begin{array}{l}4.7 \\
4.0 \\
3.8 \\
3.4 \\
3.0\end{array}$ & $\begin{array}{l}2.3 \\
2.2 \\
3.0 \\
2.7 \\
2.1\end{array}$ & $\begin{array}{l}1.3 \\
1.2 \\
1.1 \\
1.1 \\
1.2\end{array}$ \\
\hline $\begin{array}{l}26 \\
28 \\
30\end{array}$ & $\begin{array}{l}68 \\
53 \\
24 \\
14.5 \\
10.2 \\
7.9\end{array}$ & $\begin{array}{l}4.7 \\
4.4 \\
6.0 \\
3.8 \\
3.5 \\
3.4\end{array}$ & $\begin{array}{l}3.0 \\
2.8 \\
3.0 \\
3.0 \\
2.6\end{array}$ & $\begin{array}{r}1.1 \\
19.1 \\
6.4 \\
2.3 \\
1.9 \\
1.6\end{array}$ & $\begin{array}{l}5.3 \\
3.9 \\
3.6 \\
3.3 \\
3.1\end{array}$ & $\begin{array}{r}20 \\
9.2 \\
10.3 \\
11.0 \\
6.5 \\
5.7\end{array}$ & $\begin{array}{r}7.3 \\
6.0 \\
10.6 \\
6.0 \\
4.8 \\
4.3\end{array}$ & $\begin{array}{c}10.0 \\
8.5 \\
41\end{array}$ & $\begin{array}{l}2.6 \\
2.5 \\
2.3 \\
2.2 \\
2.0 \\
2.0\end{array}$ & \begin{tabular}{l|}
2.7 \\
2.6 \\
2.3 \\
2.2 \\
2.2 \\
-
\end{tabular} & $\begin{array}{l}1.9 \\
2.1 \\
3.0 \\
2.6 \\
1.6 \\
1.5\end{array}$ & 1.1 \\
\hline \multirow{2}{*}{\multicolumn{4}{|c|}{ Month }} & \multicolumn{4}{|c|}{ Million gallons a day } & \multirow{2}{*}{\multicolumn{2}{|c|}{$\begin{array}{l}\text { Second- } \\
\text { feet } \\
\text { (mean) }\end{array}$}} & \multicolumn{3}{|c|}{ Total runœoff } \\
\hline & & & & Maximum & \multicolumn{2}{|c|}{ Minimum } & Mean & & & $\begin{array}{l}\text { Million } \\
\text { gallons }\end{array}$ & \multicolumn{2}{|c|}{ Acre-feet } \\
\hline \multicolumn{4}{|c|}{$\begin{array}{l}\text { July } \\
\text { August } \\
\text { September } \\
\text { October } \\
\text { November } \\
\text { December } \\
\text { January } \\
\text { February } \\
\text { March } \\
\text { April } \\
\text { May } \\
\text { June }\end{array}$} & $\begin{array}{l}68 \\
33 \\
35 \\
19.1 \\
32 \\
57 \\
75 \\
93 \\
61 \\
46 \\
9.1 \\
7.4\end{array}$ & & $\begin{array}{l}3.6 \\
3.4 \\
2.6 \\
1.1 \\
1.6 \\
2.5 \\
4.3 \\
2.4 \\
2.0 \\
1.6 \\
1.5\end{array}$ & $\begin{array}{r}14 . \\
7.8 \\
7.8 \\
2.8 \\
9.0 \\
16 . \\
11 . \\
22.2 \\
14.2 \\
7.8 \\
3.2 \\
1.6\end{array}$ & & $\begin{array}{l}21.7 \\
11.7 \\
12.1 \\
3.91 \\
14.0 \\
25.2 \\
17.5 \\
34.3 \\
22.0 \\
12.3 \\
5.01 \\
2.54\end{array}$ & $\begin{array}{l}433 \\
234 \\
234 \\
78.4 \\
272 \\
\mathbf{5 0 6} \\
352 \\
622 \\
441 \\
239 \\
100 \\
49.2\end{array}$ & & $\begin{array}{r}1,330 \\
718 \\
720 \\
241 \\
834 \\
1,550 \\
1,080 \\
1,910 \\
1,350 \\
732 \\
308 \\
151\end{array}$ \\
\hline \multicolumn{2}{|l|}{ The year.. } & & & 93 & & - & 9.7 & & 15. 1 & 3,560 & & 10,900 \\
\hline
\end{tabular}




\section{HOTOPOU STREAM NEAR HUELO, MAUI}

Location.-Water-stage recorder just above New Hamakua ditch crossing, $23^{4}$ miles west of Kailua, and $21 / 4$ miles southwest of Huelo; elevation about 1,250 feet.

Drainage area.-1.0 square mile.

Records avail able. - December, 1910, to June, 1929.

Extremes.-Maximum discharge during year, 201 million gallons a day or 311 second-feet Dec. 13 (gage height, 3.44 feet); minimum 0.6 million gallons a day or 0.9 second-foot several days in October.

1910-1929: Maximum discharge, 658 million gallons a day or 1,020 second-feet Feb. 1, 1922 (gage height, 5.50 feet); minimum, 0.15 million gallons a day or 0.23 second-foot July 14, 1920 .

REMARKs.-Records good. No diversions.

Discharge, in million gallons a day, 1928-29

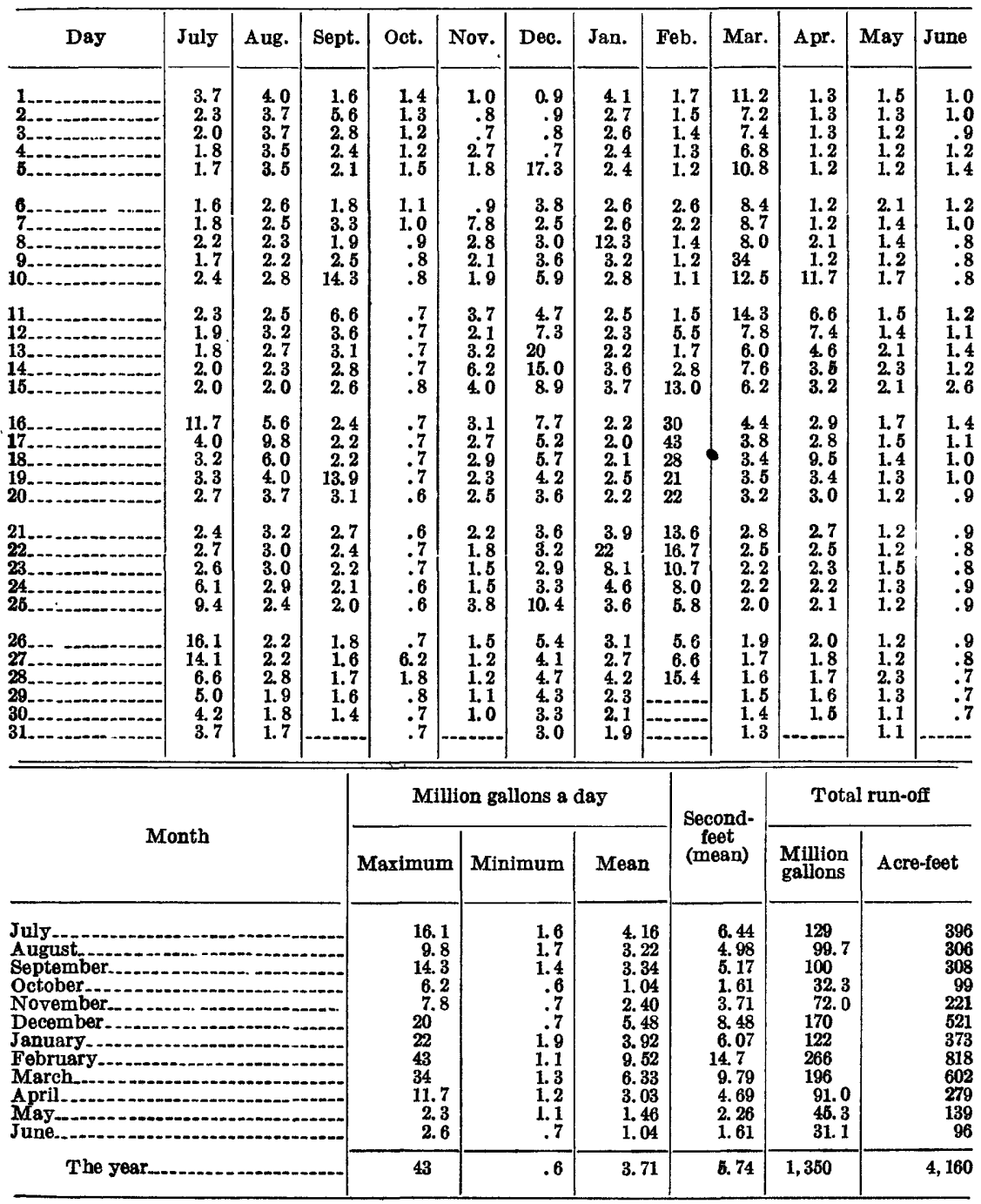




\section{WAHOA DITCH AT HONOPOU, NEAR HUELO, MAUI}

Location.-Water-stage recorder 100 feet below intake at Honopou Stream, half a mile west of Lupi, and $2 \frac{1}{2}$ miles west of Kailua.

Records AVAILABLE. - November, 1922, to June, 1929.

EXTREMES.- Not known for this year, owing to missing record.

1923-1928: Maximum discharge, 171 million gallons a day or 265 secondfeet Apr. 6-7, 1928 (gage height, 5.72 feet); minimum, 12.2 million gallons a day or 18.9 second-feet Jan. 13, 1927 .

Remarks.-Records fair. Discharges for July 4-6, July 13 to Aug. 24, Aug. 29 to Oct. 13, and Oct. 20 to Feb. 25 taken from an average of twice daily readings of staff gage by an employee of East Maui Irrigation Co.

Discharge, in million gallons a day, 1928-29

\begin{tabular}{|c|c|c|c|c|c|c|c|c|c|c|c|c|}
\hline Day & July & Aug. & Sept. & Oct. & Nov. & Dec. & Jan. & Feb. & Mar. & Apr. & May & June \\
\hline $\begin{array}{l}1 \\
2 \\
3 \\
4 \\
6\end{array}$ & $\begin{array}{l}162 \\
158 \\
154 \\
150 \\
134\end{array}$ & $\begin{array}{l}162 \\
158 \\
162 \\
154 \\
158\end{array}$ & $\begin{array}{l}122 \\
154 \\
158 \\
162 \\
154\end{array}$ & $\begin{array}{r}92 \\
138 \\
111 \\
103 \\
138\end{array}$ & $\begin{array}{r}96 \\
92 \\
74 \\
67 \\
142\end{array}$ & $\begin{array}{l}88 \\
85 \\
81 \\
78 \\
85\end{array}$ & $\begin{array}{l}162 \\
142 \\
158 \\
150 \\
142\end{array}$ & $\begin{array}{l}99 \\
92 \\
88 \\
81 \\
78\end{array}$ & $\begin{array}{l}170 \\
170 \\
170 \\
170 \\
170\end{array}$ & $\begin{array}{l}78 \\
74 \\
82 \\
86 \\
93\end{array}$ & $\begin{array}{l}88 \\
71 \\
64 \\
64 \\
68\end{array}$ & $\begin{array}{r}67 \\
64 \\
64 \\
123 \\
158\end{array}$ \\
\hline $\begin{array}{r}6 \\
7 \ldots- \\
8 \\
9 \\
0 \\
10\end{array}$ & $\begin{array}{l}118 \\
146 \\
158 \\
154 \\
158\end{array}$ & $\begin{array}{l}150 \\
146 \\
138 \\
130 \\
158\end{array}$ & $\begin{array}{l}142 \\
154 \\
150 \\
158 \\
166\end{array}$ & $\begin{array}{r}118 \\
103 \\
96 \\
81 \\
74\end{array}$ & $\begin{array}{l}103 \\
166 \\
154 \\
154 \\
158\end{array}$ & $\begin{array}{l}166 \\
158 \\
150 \\
166 \\
166\end{array}$ & $\begin{array}{r}154 \\
150 \\
158 \\
150 \\
96\end{array}$ & $\begin{array}{r}111 \\
158 \\
118 \\
88 \\
81\end{array}$ & $\begin{array}{l}170 \\
170 \\
170 \\
174 \\
170\end{array}$ & $\begin{array}{r}74 \\
67 \\
92 \\
144 \\
116\end{array}$ & $\begin{array}{r}144 \\
134 \\
142 \\
93 \\
111\end{array}$ & $\begin{array}{r}152 \\
103 \\
78 \\
71 \\
71\end{array}$ \\
\hline $\begin{array}{l}11 \\
12 \\
13\end{array}$ & $\begin{array}{l}158 \\
150 \\
154 \\
158 \\
154\end{array}$ & $\begin{array}{l}158 \\
158 \\
154 \\
150 \\
142\end{array}$ & $\begin{array}{l}166 \\
158 \\
150 \\
142 \\
130\end{array}$ & $\begin{array}{r}71 \\
67 \\
64 \\
81 \\
131\end{array}$ & $\begin{array}{l}170 \\
162 \\
162 \\
166 \\
166\end{array}$ & $\begin{array}{l}166 \\
170 \\
166 \\
170 \\
170\end{array}$ & $\begin{array}{l}126 \\
118 \\
118 \\
166 \\
166\end{array}$ & $\begin{array}{r}78 \\
170 \\
138 \\
138 \\
174\end{array}$ & $\begin{array}{l}170 \\
170 \\
166 \\
168 \\
166\end{array}$ & $\begin{array}{l}130 \\
170 \\
170 \\
166 \\
162\end{array}$ & $\begin{array}{l}150 \\
144 \\
158 \\
158 \\
150\end{array}$ & $\begin{array}{l}109 \\
102 \\
134 \\
102 \\
162\end{array}$ \\
\hline 18 & $\begin{array}{l}154 \\
162 \\
158 \\
162 \\
158\end{array}$ & $\begin{array}{l}166 \\
174 \\
166 \\
158 \\
192\end{array}$ & $\begin{array}{l}138 \\
122 \\
118 \\
170 \\
150\end{array}$ & $\begin{array}{l}92 \\
74 \\
71 \\
72 \\
60\end{array}$ & $\begin{array}{l}158 \\
150 \\
154 \\
154 \\
150\end{array}$ & $\begin{array}{l}170 \\
162 \\
166 \\
158 \\
158\end{array}$ & $\begin{array}{l}154 \\
130 \\
134 \\
158 \\
154\end{array}$ & $\begin{array}{l}174 \\
178 \\
166 \\
146 \\
178\end{array}$ & $\begin{array}{l}166 \\
158 \\
150 \\
151 \\
143\end{array}$ & $\begin{array}{l}158 \\
150 \\
149 \\
166 \\
154\end{array}$ & $\begin{array}{r}138 \\
118 \\
103 \\
92 \\
85\end{array}$ & $\begin{array}{r}149 \\
105 \\
96 \\
118 \\
74\end{array}$ \\
\hline $\begin{array}{l}21 \\
22 \\
24 \\
25\end{array}$ & $\begin{array}{l}154 \\
154 \\
158 \\
170 \\
170\end{array}$ & $\begin{array}{l}158 \\
150 \\
154 \\
162 \\
150\end{array}$ & $\begin{array}{r}134 \\
118 \\
107 \\
99 \\
92\end{array}$ & $\begin{array}{r}71 \\
107 \\
67 \\
60 \\
57\end{array}$ & $\begin{array}{l}158 \\
150 \\
138 \\
138 \\
146\end{array}$ & $\begin{array}{l}158 \\
154 \\
150 \\
154 \\
166\end{array}$ & $\begin{array}{l}154 \\
178 \\
174 \\
166 \\
158\end{array}$ & $\begin{array}{l}174 \\
178 \\
166 \\
166 \\
166\end{array}$ & $\begin{array}{l}140 \\
126 \\
113 \\
111 \\
111\end{array}$ & $\begin{array}{l}138 \\
122 \\
114 \\
117 \\
103\end{array}$ & \begin{tabular}{r|}
81 \\
79 \\
140 \\
130 \\
88
\end{tabular} & $\begin{array}{l}71 \\
67 \\
64 \\
66 \\
74\end{array}$ \\
\hline 28 & $\begin{array}{l}170 \\
174 \\
170 \\
166 \\
162 \\
162\end{array}$ & $\begin{array}{l}142 \\
134 \\
150 \\
130 \\
122 \\
111\end{array}$ & $\begin{array}{r}85 \\
81 \\
111 \\
146 \\
99\end{array}$ & \begin{tabular}{r|r}
54 & \\
107 & \\
150 & \\
111 & \\
85 & \\
96 & $\ldots$
\end{tabular} & $\begin{array}{r}134 \\
126 \\
107 \\
99 \\
103\end{array}$ & $\begin{array}{l}166 \\
162 \\
162 \\
166 \\
158 \\
150\end{array}$ & \begin{tabular}{l|l}
150 & \\
138 & \\
146 & \\
138 & $\ldots$ \\
118 & -- \\
107 & -
\end{tabular} & $\begin{array}{l}162 \\
166 \\
170 \\
\\
\end{array}$ & $\begin{array}{r}107 \\
99 \\
96 \\
88 \\
85 \\
81\end{array}$ & $\begin{array}{c}92 \\
92 \\
81 \\
81 \\
78 \\
-\end{array}$ & $\begin{array}{r}78 \\
108 \\
132 \\
103 \\
81 \\
74\end{array}$ & $\begin{array}{l}67 \\
57 \\
54 \\
51 \\
56\end{array}$ \\
\hline \multirow{2}{*}{\multicolumn{4}{|c|}{ Month }} & \multicolumn{4}{|c|}{ Million gallons a day } & \multirow{2}{*}{\multicolumn{2}{|c|}{$\begin{array}{c}\text { Second- } \\
\text { feet } \\
\text { (mean) }\end{array}$}} & \multicolumn{3}{|c|}{ Tatal run-off } \\
\hline & & & & Maximum & a Min & num & Mean & & & $\begin{array}{l}\text { Million } \\
\text { gallons }\end{array}$ & Acr & e-feet \\
\hline \multicolumn{4}{|c|}{$\begin{array}{l}\text { July } \\
\text { A ugust } \\
\text { September } \\
\text { October } \\
\text { November } \\
\text { December } \\
\text { January } \\
\text { February } \\
\text { March } \\
\text { April } \\
\text { May }\end{array}$} & $\begin{array}{l}174 \\
174 \\
170 \\
150 \\
170 \\
170 \\
178 \\
178 \\
174 \\
170 \\
158 \\
162\end{array}$ & & \begin{tabular}{r|}
118 \\
111 \\
81 \\
54 \\
67 \\
78 \\
96 \\
78 \\
81 \\
67 \\
64 \\
51
\end{tabular} & $\begin{array}{c}157 \\
151 \\
135 \\
90.4 \\
137 \\
149 \\
146 \\
139 \\
144 \\
117 \\
109 \\
91.0\end{array}$ & & $\begin{array}{l}243 \\
234 \\
209 \\
140 \\
212 \\
231 \\
226 \\
215 \\
223 \\
181 \\
169 \\
141\end{array}$ & $\begin{array}{l}4,870 \\
4,670 \\
4,040 \\
2,800 \\
4,100 \\
4,620 \\
4,510 \\
3,880 \\
4,470 \\
3,500 \\
3,370 \\
2,730\end{array}$ & & $\begin{array}{r}14.900 \\
14,400 \\
12,400 \\
8,600 \\
12,600 \\
14,200 \\
13,900 \\
11,900 \\
13,700 \\
10,800 \\
10,400 \\
8,380\end{array}$ \\
\hline \multicolumn{2}{|l|}{ The year... } & & & 178 & & 51 & 130 & & 201 & 47,600 & & 146,000 \\
\hline
\end{tabular}


INW HAMAKUA DTCH AT HONOPOU, NEAR HUEIO, MAUI

Location.-Water-stage recorder 600 feet below Honopou Stream crossing, 15 feet above tunnel portal, and $21 / 2$ miles west of Kailua.

Records AVAIL ABle.-January, 1918, to June, 1929.

Extremes.- Maximum discharge during year, 127 million gallons a day or 196 second-feet Sept. 19 (gage height, 5.68 feet); minimum, 0.4 million gallons a day or 0.6 second-foot June 29 .

1918-1929: Maximum discharge, 134 million gallons a day or 207 secondfeet Aug. 5, 1926 (gage height, 5.98 feet); minimum, 0.07 million gallons a day or 0.11 second-foot Aug. 7, 1923.

REMARKs.- Records good for ordinary stages, fair for high stages, and poor for estimated periods. New Hamakua ditch diverts from streams between Waikamoi and Halehaku above Center and Lowrie ditches. Regulated by gates and spillways.

Discharge, in million gallons a day, 1928-29

\begin{tabular}{|c|c|c|c|c|c|c|c|c|c|c|c|c|}
\hline D & uly & ug. & Sept. & Oct. & Nov. & Dec. & Jan. & Feb. & Mar. & Apr. & May & June \\
\hline 4 & $\begin{array}{c}76 \\
57 \\
39 \\
21 \\
4.0\end{array}$ & $\begin{array}{l}39 \\
62 \\
51 \\
58\end{array}$ & $\begin{array}{l}3.0 \\
55 \\
65 \\
66 \\
46\end{array}$ & $\begin{array}{r}8.8 \\
33 \\
1.6 \\
2.2 \\
39\end{array}$ & $\begin{array}{r}3.6 \\
2.0 \\
1.7 \\
40\end{array}$ & $\begin{array}{r}1.0 \\
1.0 \\
.9 \\
.8 \\
\times 49^{.8}\end{array}$ & $\begin{array}{l}59 \\
42 \\
28\end{array}$ & $\begin{array}{l}\text { 3.5 } \\
\text { 3.2 } \\
3.2 \\
3.0 \\
2.8\end{array}$ & $\begin{array}{l}69 \\
71 \\
77\end{array}$ & $\begin{array}{l}.7 \\
.8 \\
.7\end{array}$ & $\begin{array}{l}0.8 \\
.6 \\
.6 \\
.5 \\
.5\end{array}$ & $\begin{array}{r}.6 \\
.6 \\
18.5 \\
38.6\end{array}$ \\
\hline $\begin{array}{l}7 \ldots \\
8 \cdots \\
80\end{array}$ & $\begin{array}{l}1.8 \\
31 \\
64 \\
35 \\
57\end{array}$ & \begin{tabular}{l|}
31 \\
28 \\
15.2 \\
4.8 \\
55
\end{tabular} & $\begin{array}{l}31 \\
48 \\
39 \\
57 \\
78\end{array}$ & $\begin{array}{r}12.3 \\
1.3 \\
1.2 \\
1.0 \\
.9\end{array}$ & $\begin{array}{l}1.2 \\
64 \\
54 \\
56 \\
62\end{array}$ & $\begin{array}{r}678 \\
88 \\
38 \\
73 \\
72\end{array}$ & $\begin{array}{l}\mathbf{5 9} \\
41 \\
79 \\
28 \\
\mathbf{3 0}\end{array}$ & \begin{tabular}{c|}
20 \\
48 \\
7.7 \\
3.3 \\
3.1
\end{tabular} & $\begin{array}{l}74 \\
89 \\
80\end{array}$ & $\begin{array}{r}.6 \\
1.4 \\
50 \\
2.3 \\
46\end{array}$ & $\begin{array}{l}40 \\
29 \\
27 \\
1.0 \\
4.8\end{array}$ & 9. \\
\hline (n).................. & $\begin{array}{l}54 \\
22 \\
26 \\
44 \\
32\end{array}$ & $\begin{array}{l}50 \\
41 \\
56 \\
40 \\
26\end{array}$ & $\begin{array}{r}80 \\
55 \\
34 \\
13.4 \\
\times 16.5\end{array}$ & $\begin{array}{r}.9 \\
.9 \\
.9 \\
23\end{array}$ & $\begin{array}{l}79 \\
62 \\
66 \\
73 \\
71\end{array}$ & $\begin{array}{l}75 \\
81 \\
83 \\
93 \\
84\end{array}$ & $\begin{array}{r}5.5 \\
2.8 \\
65\end{array}$ & $\begin{array}{l}3.1 \\
70 \\
20 \\
33 \\
87\end{array}$ & $\begin{array}{l}80 \\
72 \\
58 \\
62 \\
54\end{array}$ & $\begin{array}{l}74 \\
77 \\
64 \\
63 \\
49\end{array}$ & $\begin{array}{l}49 \\
37 \\
56 \\
56 \\
45\end{array}$ & 14 \\
\hline$\cdots$ & $\begin{array}{l}64 \\
73 \\
62 \\
65 \\
45\end{array}$ & $\begin{array}{l}68 \\
87 \\
77 \\
54 \\
57\end{array}$ & $\begin{array}{l}28 \\
11.3 \\
8.9 \\
98 \\
55\end{array}$ & $\begin{array}{r}1.1 \\
.9 \\
.8 \\
.9 \\
.7\end{array}$ & $\begin{array}{l}\mathbf{5 6} \\
\mathbf{3 4} \\
\mathbf{5 5} \\
\mathbf{5 0} \\
\mathbf{3 8}\end{array}$ & $\begin{array}{l}81 \\
52 \\
63 \\
40 \\
41\end{array}$ & 8 & $\begin{array}{l}90 \\
91 \\
71 \\
47 \\
86\end{array}$ & $\begin{array}{l}47 \\
30 \\
21 \\
18.7 \\
11.4\end{array}$ & \begin{tabular}{c|}
25 \\
27 \\
63 \\
38 \\
8.7
\end{tabular} & $\begin{array}{l}9.4 \\
1.4 \\
.8 \\
.8\end{array}$ & i. \\
\hline (2) & $\begin{array}{l}19.3 \\
50 \\
56 \\
75 \\
88\end{array}$ & $\begin{array}{l}53 \\
54 \\
49 \\
64 \\
41\end{array}$ & $\begin{array}{r}19.4 \\
8.9 \\
8.4 \\
7.8 \\
7.3\end{array}$ & $\begin{array}{l}.7 \\
1.8 \\
.8 \\
.7 \\
.6\end{array}$ & \begin{tabular}{l|}
59 \\
35 \\
19.2 \\
21 \\
22
\end{tabular} & $\begin{array}{l}41 \\
31 \\
18.6 \\
40 \\
69\end{array}$ & 75 & $\begin{array}{l}68 \\
83 \\
35 \\
40 \\
44\end{array}$ & $\begin{array}{l}9.6 \\
2.0 \\
1.6 \\
1.5 \\
1.4\end{array}$ & $\begin{array}{r}1.6 \\
1.3 \\
1.0 \\
1.5 \\
.9\end{array}$ & $\begin{array}{r}.6 \\
17.6 \\
17.8 \\
.8\end{array}$ & 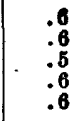 \\
\hline $\begin{array}{l}26 \\
27 \\
28 \\
30 \\
31\end{array}$ & $\begin{array}{l}89 \\
89 \\
76 \\
54 \\
37 \\
24\end{array}$ & $\begin{array}{r}26 \\
15.1 \\
45 \\
9.5 \\
2.8 \\
1.6\end{array}$ & $\begin{array}{r}7.3 \\
6.8 \\
6.2 \\
35 \\
1.7\end{array}$ & $\begin{array}{l}40^{.6} \\
50 \\
2.8 \\
1.2 \\
2.0\end{array}$ & $\begin{array}{r}13.9 \\
1.5 \\
1.2 \\
1.2 \\
1.2\end{array}$ & $\begin{array}{l}71 \\
42 \\
59 \\
62 \\
32 \\
23\end{array}$ & $\begin{array}{c}8.8 \\
41 \\
9.9 \\
4.0 \\
3.6\end{array}$ & $\begin{array}{r}54 \\
46 \\
81 \\
\\
\end{array}$ & $\begin{array}{r}1.2 \\
1.0 \\
.9 \\
.8 \\
.8 \\
.8\end{array}$ & $\begin{array}{l}.8 \\
.8 \\
.7 \\
.7 \\
.7\end{array}$ & $\begin{array}{r}12.7 \\
26 \\
3.8 \\
.8 \\
.7\end{array}$ & \\
\hline & \multicolumn{4}{|c|}{ Million gallons a day } & \multirow{2}{*}{\multicolumn{2}{|c|}{$\begin{array}{l}\text { Second- } \\
\text { feet } \\
\text { (mean) }\end{array}$}} & \multicolumn{3}{|c|}{ Total run-ofi } \\
\hline & & & & Maximum & \multicolumn{2}{|c|}{ a Minimum } & Mean & & & $\begin{array}{l}\text { Million } \\
\text { gallons }\end{array}$ & \multicolumn{2}{|c|}{ Acre-feet } \\
\hline \multicolumn{4}{|c|}{ 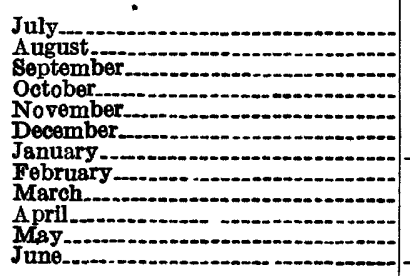 } & $\begin{array}{l}89 \\
87 \\
98 \\
50 \\
79 \\
93 \\
91 \\
89 \\
87 \\
56\end{array}$ & $\begin{array}{l}9 \\
7 \\
8 \\
0 \\
0 \\
3 \\
i \\
9 \\
7 \\
6\end{array}$ & \begin{tabular}{r}
1.8 \\
1.6 \\
1.7 \\
.6 \\
.7 \\
\hdashline .8 \\
.8 \\
.8 \\
.6 \\
.5
\end{tabular} & $\begin{array}{c}49.4 \\
42.7 \\
33.2 \\
7.54 \\
34.8 \\
50.1 \\
33.5 \\
41.0 \\
39.8 \\
19.8 \\
14.3 \\
7.07\end{array}$ & & $\begin{array}{l}76.4 \\
66.1 \\
51.4 \\
11.7 \\
53.8 \\
77.5 \\
51.8 \\
63.4 \\
61.6 \\
30.6 \\
22.1 \\
10.9\end{array}$ & $\begin{array}{r}1,530 \\
1,320 \\
997 \\
234 \\
1,040 \\
1,550 \\
1,040 \\
1,150 \\
1,230 \\
593 \\
442 \\
212\end{array}$ & \multicolumn{2}{|r|}{$\begin{array}{r}4,700 \\
4,060 \\
3,060 \\
717 \\
3,200 \\
4,770 \\
3,190 \\
3,520 \\
3,790 \\
1,820 \\
1,360 \\
651\end{array}$} \\
\hline \multicolumn{3}{|l|}{ The : } & & 98 & & .4 & 31.1 & & 48.1 & 1,300 & & 80 \\
\hline
\end{tabular}


KAUHIKOA DITCH AT OPANA WEIR, NEAR HUELO, MAUI

LOCATION.-Water-stage recorder a short distance below crossing of Opana Stream and 8 miles by road west of Huelo post office.

RecoRDS AVAILABLE.-January, 1910, to December, 1928 (discontinued).

ExTREMEs.-See monthly discharge table for maximum and minimum daily discharges.

REMARKS.-Daily-discharge record furnished by East Maui Irrigation Co. Diverts water at elevation 900 feet from all streams between Halehaku and Maliko. Regulated by gates.

Discharge, in million gallons a day, 1928

\begin{tabular}{|c|c|c|c|c|c|c|c|c|c|c|c|c|c|}
\hline Day & July & Aug. & Sept. & Oct. & Nov. & Dec & Day & July & Aug. & Sept. & Oct. & Nov. & Dec. \\
\hline $\begin{array}{l}1 \\
2 \\
3 \\
4 \\
5 \\
5\end{array}$ & $\begin{array}{c}70 \\
52 \\
32 \\
15.3 \\
3.8\end{array}$ & $\begin{array}{l}5.4 \\
45 \\
51 \\
50 \\
46\end{array}$ & $\begin{array}{l}6.7 \\
56 \\
57 \\
55 \\
39\end{array}$ & $\begin{array}{l}19.0 \\
21 \\
2.5 \\
10.7 \\
35\end{array}$ & $\begin{array}{r}5.8 \\
2.3 \\
2.0 \\
23 \\
19.5\end{array}$ & $\begin{array}{r}2 . \\
2 . \\
2 . \\
2 . \\
65\end{array}$ & \begin{tabular}{l|ll}
2 & 16 \\
2 & 17 \\
0 & 18 \\
0 & 19 \\
& 20
\end{tabular} & $\left\{\begin{array}{l}75 \\
55\end{array}\right.$ & $\begin{array}{l}64 \\
85 \\
66 \\
45 \\
56\end{array}$ & $\begin{array}{l}13.4 \\
4.0 \\
16.2 \\
72 \\
26\end{array}$ & $\begin{array}{l}2.0 \\
1.8 \\
1.8 \\
1.8 \\
1.7\end{array}$ & $\begin{array}{l}46 \\
35 \\
56 \\
42 \\
45\end{array}$ & $\begin{array}{l}78 \\
47 \\
59 \\
40 \\
38\end{array}$ \\
\hline 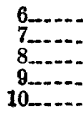 & $\begin{array}{l}2.8 \\
39 \\
56 \\
32 \\
56\end{array}$ & $\begin{array}{l}28 \\
24 \\
12.3 \\
9.7 \\
53\end{array}$ & $\begin{array}{l}28 \\
47 \\
35 \\
51 \\
81\end{array}$ & $\begin{array}{l}6.1 \\
2.4 \\
2.1 \\
2.0 \\
1.8\end{array}$ & $\begin{array}{l}3.9 \\
77 \\
52 \\
53 \\
62\end{array}$ & $\begin{array}{l}74 \\
50 \\
46 \\
68 \\
69\end{array}$ & $\begin{array}{l}21 \ldots \ldots \\
22 \\
23 \\
24 \\
25\end{array}$ & $\begin{array}{l}14.1 \\
54 \\
46 \\
82 \\
88\end{array}$ & $\begin{array}{l}43 \\
44 \\
51 \\
54 \\
34\end{array}$ & $\begin{array}{l}6.8 \\
3.4 \\
3.0 \\
2.8 \\
2.7\end{array}$ & $\begin{array}{l}1.6 \\
2.5 \\
1.5 \\
1.4 \\
1.4\end{array}$ & \begin{tabular}{l|}
$\mathbf{5 1}$ \\
$\mathbf{2 9}$ \\
21 \\
16.2 \\
$\mathbf{2 9}$
\end{tabular} & $\begin{array}{l}37 \\
28 \\
14.5 \\
41 \\
70\end{array}$ \\
\hline $\begin{array}{l}11 \\
12 \\
13 \\
14 \\
15\end{array}$ & $\begin{array}{l}42 \\
17.8 \\
24 \\
44 \\
24\end{array}$ & $\begin{array}{l}46 \\
34 \\
49 \\
28 \\
35\end{array}$ & $\begin{array}{l}71 \\
46 \\
30 \\
14.8 \\
9.0\end{array}$ & $\begin{array}{r}1.8 \\
1.8 \\
1.7 \\
12.9 \\
11.4\end{array}$ & $\begin{array}{l}78 \\
61 \\
65 \\
71 \\
63\end{array}$ & $\begin{array}{l}69 \\
78 \\
85 \\
91 \\
86\end{array}$ & $\begin{array}{l}26 \\
27 \ldots \ldots \\
28 \\
29 \\
30 \ldots \\
31 \ldots\end{array}$ & $\begin{array}{l}94 \\
88 \\
52 \\
70 \\
33 \\
25\end{array}$ & $\begin{array}{c}21 \\
23 \\
32 \\
7.5 \\
3.2 \\
2.6\end{array}$ & $\begin{array}{r}2.6 \\
2.4 \\
5.7 \\
32 \\
2.4\end{array}$ & $\begin{array}{c}1.3 \\
56 \\
33 \\
2.6 \\
3.2 \\
2.1\end{array}$ & $\begin{array}{l}6.7 \\
3.0 \\
2.8 \\
2.5 \\
2.4\end{array}$ & $\begin{array}{l}59 \\
38 \\
58 \\
47 \\
26 \\
24\end{array}$ \\
\hline \multirow{2}{*}{\multicolumn{5}{|c|}{ Month }} & \multicolumn{4}{|c|}{ Million gallons a day } & \multirow{2}{*}{\multicolumn{2}{|c|}{$\begin{array}{c}\text { Second- } \\
\text { feet } \\
\text { (mean) }\end{array}$}} & \multicolumn{3}{|c|}{ Total run-off } \\
\hline & & & & & Maxim & & Minimum & Mean & & & $\begin{array}{l}\text { Million } \\
\text { gallons }\end{array}$ & Acre & -feet \\
\hline \multicolumn{5}{|c|}{$\begin{array}{l}\text { July } \\
\text { August. } \\
\text { September } \\
\text { October } \\
\text { November } \\
\text { December }\end{array}$} & & $\begin{array}{l}94 \\
85 \\
81 \\
56 \\
78 \\
91\end{array}$ & $\begin{array}{l}2.8 \\
2.6 \\
2.4 \\
1.3 \\
2.0 \\
2.0\end{array}$ & $\begin{array}{l}46.8 \\
37.0 \\
27.4 \\
8.00 \\
34.2 \\
48.1\end{array}$ & & $\begin{array}{l}72.4 \\
57.2 \\
42.4 \\
12.4 \\
52.9 \\
74.4\end{array}$ & $\begin{array}{r}1,460 \\
1,150 \\
822 \\
248 \\
1,030 \\
1,490\end{array}$ & & $\begin{array}{l}4,450 \\
3,520 \\
2,520 \\
761 \\
3,150 \\
4,580\end{array}$ \\
\hline \multicolumn{5}{|c|}{ The period. } & & & & & & & 6,190 & & 19,000 \\
\hline
\end{tabular}




\section{HAIR DITCH AT MANAWAI GULCH, NEAR PEAHI, MAOI}

Location.-Water-stage recorder in bottom of western branch of Manawai Gulch, just west of Keaaula-Opana boundary and 1 mile north of Peahi.

Records available. - January, 1910, to December, 1928 (discontinued).

EXTREMES. - See monthly-discharge table for maximum and minimum daily discharges.

Remarks.-Daily-discharge record furnished by East Maui Irrigation Co. Regulated by gates at frequent intervals. Diverts water at elevation 250 feet from all streams between Kailua Stream and Maliko Gulch.

Discharge, in million gallons a day, 1928

\begin{tabular}{|c|c|c|c|c|c|c|c|c|c|c|c|c|c|}
\hline Day & July & Aug. & Sept. & Oct. & Nov. & Dec. & Day & July & Aug. & Sept. & Oct. & Nov. & Dec. \\
\hline $\begin{array}{l}1 \\
2 \\
3 \\
4 \\
4 \\
5\end{array} \ldots$ & $\begin{array}{l}60 \\
37 \\
31 \\
28 \\
24\end{array}$ & $\begin{array}{l}47 \\
32 \\
38 \\
36 \\
37\end{array}$ & $\begin{array}{l}9.8 \\
49 \\
49 \\
42 \\
31\end{array}$ & $\begin{array}{l}21 \\
29 \\
18.2 \\
11.6 \\
20\end{array}$ & $\begin{array}{l}24 \\
15.5 \\
19.2 \\
24 \\
30\end{array}$ & \begin{tabular}{r|}
7.9 \\
7.4 \\
7.1 \\
6.6 \\
57
\end{tabular} & \begin{tabular}{l||l}
9 & 16 \\
4 & 17 \\
1 & 18 \\
6 & 19 \\
19 & $\ldots$ \\
20 & $\ldots$
\end{tabular} & $\begin{array}{l}53 \\
53 \\
36 \\
38 \\
44\end{array}$ & $\begin{array}{l}53 \\
60 \\
51 \\
38 \\
48\end{array}$ & $\begin{array}{l}17.0 \\
14.9 \\
28 \\
60 \\
32\end{array}$ & $\begin{array}{l}7.2 \\
6.6 \\
6.5 \\
8.4 \\
6.1\end{array}$ & $\begin{array}{l}31 \\
36 \\
57 \\
43 \\
45\end{array}$ & $\begin{array}{l}70 \\
46 \\
52 \\
34 \\
31\end{array}$ \\
\hline $\begin{array}{r}6 \\
7 \\
8 \\
9 \\
9 \\
10\end{array}$ & $\begin{array}{l}18.6 \\
32 \\
49 \\
28 \\
38\end{array}$ & $\begin{array}{l}13.2 \\
38 \\
29 \\
12.6 \\
15.8\end{array}$ & $\begin{array}{l}28 \\
41 \\
33 \\
41 \\
64\end{array}$ & $\begin{array}{r}10.8 \\
9.2 \\
9.0 \\
7.6 \\
7.2\end{array}$ & $\begin{array}{l}9.8 \\
70 \\
36 \\
41 \\
51\end{array}$ & $\begin{array}{l}71 \\
46 \\
32 \\
66 \\
68\end{array}$ & $\begin{array}{l}21 \\
22 \\
23 \\
24 \\
25\end{array}$ & $\begin{array}{l}24 \\
42 \\
39 \\
64 \\
72\end{array}$ & $\begin{array}{l}41 \\
44 \\
45 \\
41 \\
22\end{array}$ & $\begin{array}{l}22 \\
14.8 \\
19.6 \\
20 \\
21\end{array}$ & $\begin{array}{l}5.6 \\
6.1 \\
5.1 \\
5.0 \\
4.6\end{array}$ & $\begin{array}{l}43 \\
25 \\
22 \\
21 \\
26\end{array}$ & $\begin{array}{l}29 \\
24 \\
18.8 \\
35 \\
73\end{array}$ \\
\hline $\begin{array}{l}11 \\
12 \\
13 \\
14 \\
15\end{array}$ & $\begin{array}{l}32 \\
17.5 \\
19.3 \\
31 \\
21\end{array}$ & $\begin{array}{l}31 \\
31 \\
36 \\
25 \\
19.8\end{array}$ & $\begin{array}{l}58 \\
39 \\
34 \\
24 \\
22\end{array}$ & $\begin{array}{r}6.6 \\
6.4 \\
6.1 \\
8.7 \\
12.5\end{array}$ & $\begin{array}{l}81 \\
42 \\
54 \\
57 \\
52\end{array}$ & $\begin{array}{l}70 \\
84 \\
82 \\
94 \\
80\end{array}$ & $\begin{array}{l}26 \\
27 \\
28 \\
29 \\
30 \\
31\end{array}$ & $\begin{array}{l}95 \\
83 \\
52 \\
\mathbf{3 6} \\
\mathbf{3 5} \\
\mathbf{3 8}\end{array}$ & $\begin{array}{r}15.8 \\
15.2 \\
24 \\
12.1 \\
10.4 \\
9.8\end{array}$ & $\begin{array}{r}18.8 \\
7.8 \\
7.8 \\
17.9 \\
7.3\end{array}$ & $\begin{array}{r}4.1 \\
49 \\
34 \\
9.8 \\
9.3 \\
13.8\end{array}$ & $\begin{array}{r}11.7 \\
9.8 \\
9.0 \\
9.2 \\
9.1\end{array}$ & $\begin{array}{l}62 \\
29 \\
46 \\
55 \\
27 \\
23\end{array}$ \\
\hline \multirow{2}{*}{\multicolumn{5}{|c|}{ Month }} & \multicolumn{4}{|c|}{ Million gallons a day } & \multirow{2}{*}{\multicolumn{2}{|c|}{$\begin{array}{c}\text { Second- } \\
\text { feet } \\
\text { (mean) }\end{array}$}} & \multicolumn{3}{|c|}{ Total run-off } \\
\hline & & & & & Maxim & um & Minimum & Mean & & & $\begin{array}{l}\text { Million } \\
\text { gallons }\end{array}$ & \multicolumn{2}{|c|}{ Acre-feet } \\
\hline \multicolumn{5}{|c|}{$\begin{array}{l}\text { July } \\
\text { August } \\
\text { September } \\
\text { October } \\
\text { November } \\
\text { December }\end{array}$} & \multicolumn{2}{|r|}{$\begin{array}{l}95 \\
60 \\
64 \\
49 \\
81 \\
94\end{array}$} & $\begin{array}{r}17.5 \\
9.8 \\
7.3 \\
4.1 \\
9.0 \\
6.6\end{array}$ & $\begin{array}{l}41.0 \\
31.3 \\
29.1 \\
11.8 \\
33.5 \\
46.3\end{array}$ & & $\begin{array}{l}63.4 \\
48.4 \\
45.0 \\
18.3 \\
51.8 \\
71.6\end{array}$ & $\begin{array}{r}1,270 \\
972 \\
874 \\
365 \\
1,000 \\
1,430\end{array}$ & \multicolumn{2}{|r|}{$\begin{array}{l}3,900 \\
2,980 \\
2,680 \\
1,120 \\
3,080 \\
4,400\end{array}$} \\
\hline \multicolumn{5}{|c|}{ The period } & & & & - & & ..... & 5,910 & \multicolumn{2}{|r|}{18,200} \\
\hline
\end{tabular}

\section{MISCELIANEOUS MEASUREMENTS}

Measurements of streams and ditches on the island of Maui at other than regular gaging stations are listed below:

Miscellaneous discharge measurements on Maui, 1928-29

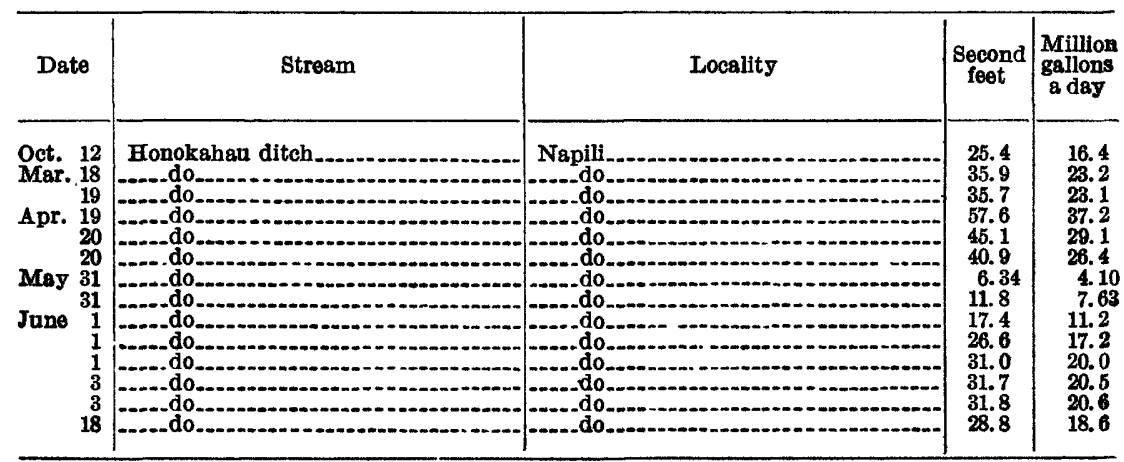




\section{ISLAND OF HAWAII}

\section{WAILUKU RIVRR ABOVE HILO BOARDING SCHOOL DITCH INTAKE, NEAR HIIO, HAWAII}

Location.-Water-stage recorder 1,000 feet above Hilo Boarding School ditch intake, three-quarters of a mile west of Reservoir No. 1, and $4 \frac{1}{2}$ miles west of Hilo.

Drainage area.-124.5 square miles.

ReCoRds avail ABle. - July, 1928, to June, 1929.

EXTREMES. - Maximum discharge during period, 13,500 million gallons a day or 20,900 second-feet Feb. 15 (gage height, 17.5 feet); minimum, 6.0 million gallons a day or 9.3 second-feet Jan. 30 .

REMARKs. - Station established June 28 , but no reliable records were obtained until July 16. Records good for ordinary stages, fair for estimated periods, and poor for high stages. Hilo waterworks diverts about 1 million gallons a day from pool at Pukamaui, three-quarters of a mile upstream.

Discharge, in million gallons a day, 1928-29

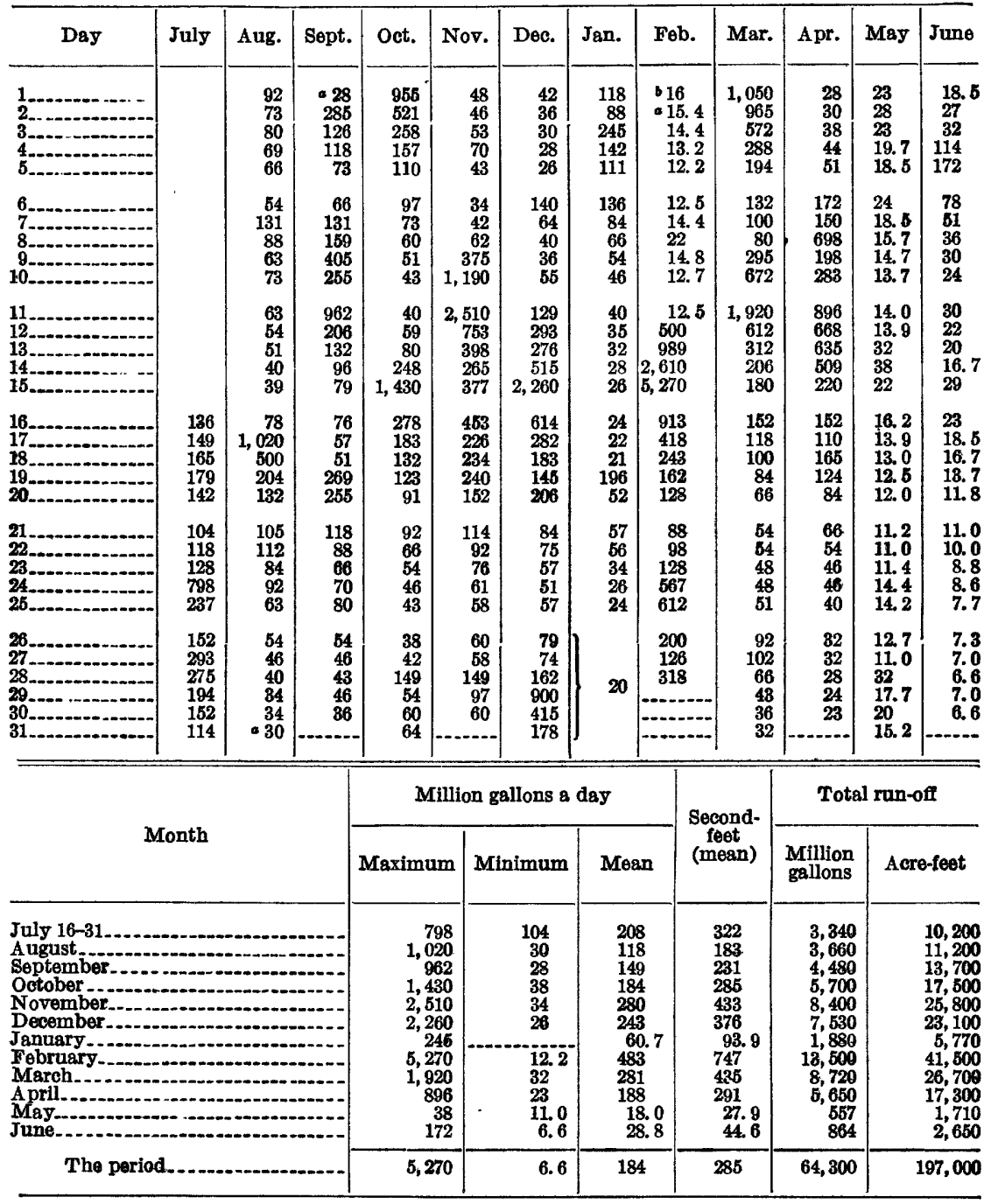


KAPEHU STREAM AT PHHONUA, IEAR HIHO, HAWAII

LOCATION.-Water-stage recorder at Piihonua, one-quarter mile above confluence with Wailuku River and 3 miles west of Hilo.

Drain age ARta. -4.9 square miles.

RicoRDs AVAILABLE. - November, 1928, to June, 1929.

Extramas. - Maximum discharge during period, 2,330 million gallons a day or 3,610 second-feet Feb. 15 (gage height, 14.65 feet); minimum; 4.3 million gallons a day or 6.6 second-feet Feb. 12 .

REMARKs.-Records good for low and medium stages; poor for extremely high stages. Station established Nov. 27, 1928. Smail diversion above station for irrigation of sugarcane.

Discharge, in million gallons a day, 1828-29

\begin{tabular}{|c|c|c|c|c|c|c|c|c|c|}
\hline \multicolumn{2}{|l|}{ Day } & Nov. & Dec. & Jan. & Feb. & Mar. & Apr. & May & June \\
\hline 1 & $0-1=-$ & & $\begin{array}{l}19.2 \\
17.5 \\
16.6 \\
16.0 \\
16.0\end{array}$ & $\begin{array}{l}39 \\
34 \\
78 \\
48 \\
36\end{array}$ & $\begin{array}{l}6.6 \\
5.9 \\
5.1 \\
4.9 \\
4.6\end{array}$ & $\begin{array}{r}154 \\
159 \\
99 \\
59 \\
45\end{array}$ & $\begin{array}{l}13.2 \\
14.0 \\
18.2 \\
21 \\
22\end{array}$ & $\begin{array}{l}15.7 \\
14.8 \\
11.8 \\
10.8 \\
10.8\end{array}$ & $\begin{array}{l}8.4 \\
10.3 \\
11.4 \\
32 \\
39\end{array}$ \\
\hline $\begin{array}{l}6 \ldots \\
7 \ldots \\
8 \ldots \\
10\end{array}$ & & & $\begin{array}{l}64 \\
27 \\
21 \\
20 \\
25\end{array}$ & $\begin{array}{l}47 \\
32 \\
27 \\
25 \\
23\end{array}$ & $\begin{array}{l}4.9 \\
6.6 \\
6.6 \\
5.1 \\
4.8\end{array}$ & $\begin{array}{r}34 \\
29 \\
27 \\
114 \\
162\end{array}$ & $\begin{array}{r}63 \\
39 \\
170 \\
61 \\
89\end{array}$ & $\begin{array}{r}10.0 \\
9.3 \\
8.6 \\
8.1 \\
7.9\end{array}$ & $\begin{array}{l}21 \\
15.4 \\
13.5 \\
13.0 \\
11.8\end{array}$ \\
\hline 18 & & & $\begin{array}{r}\dot{86} \\
64 \\
80 \\
141 \\
330\end{array}$ & $\begin{array}{l}21 \\
19.6 \\
18.6 \\
16.9 \\
15.7\end{array}$ & $\begin{array}{l}4.5 \\
59 \\
197 \\
313 \\
613\end{array}$ & $\begin{array}{r}382 \\
119 \\
75 \\
63 \\
51\end{array}$ & $\begin{array}{r}246 \\
149 \\
146 \\
107 \\
63\end{array}$ & $\begin{array}{r}8.4 \\
8.4 \\
16.8 \\
17.0 \\
11.5\end{array}$ & $\begin{array}{r}12.2 \\
9.3 \\
8.8 \\
8.1 \\
13.0\end{array}$ \\
\hline 18 16 18 & 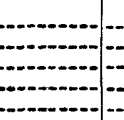 & 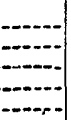 & $\begin{array}{r}104 \\
63 \\
51 \\
46 \\
34\end{array}$ & $\begin{array}{l}14.5 \\
13.5 \\
14.0 \\
40 \\
15.7\end{array}$ & $\begin{array}{r}100 \\
55 \\
42 \\
32 \\
25\end{array}$ & $\begin{array}{l}51 \\
39 \\
36 \\
32 \\
27\end{array}$ & $\begin{array}{l}46 \\
39 \\
54 \\
42 \\
32\end{array}$ & $\begin{array}{l}9.8 \\
8.8 \\
8.4 \\
8.1 \\
6.6\end{array}$ & $\begin{array}{r}10.0 \\
9.3 \\
8.6 \\
7.2 \\
6.8\end{array}$ \\
\hline 24 & 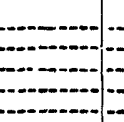 & (n) & $\begin{array}{l}32 \\
29 \\
25 \\
25 \\
25\end{array}$ & $\begin{array}{l}16.6 \\
16.3 \\
13.5 \\
12.5 \\
11.2\end{array}$ & $\begin{array}{l}21 \\
27 \\
29 \\
72 \\
58\end{array}$ & $\begin{array}{l}24 \\
24 \\
23 \\
22 \\
20\end{array}$ & $\begin{array}{l}27 \\
25 \\
23 \\
23 \\
21\end{array}$ & $\begin{array}{l}5.8 \\
6.1 \\
6.6 \\
8.1 \\
7.2\end{array}$ & $\begin{array}{l}6.6 \\
6.2 \\
5.9 \\
5.6 \\
5.5\end{array}$ \\
\hline \multicolumn{2}{|c|}{28} & $\begin{array}{r}29 \\
22 \\
22 \\
25 \\
-\cdots\end{array}$ & $\begin{array}{r}38 \\
34 \\
51 \\
202 \\
88 \\
51\end{array}$ & $\begin{array}{r}11.0 \\
12.8 \\
11.2 \\
10.2 \\
9.0 \\
7.9\end{array}$ & 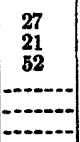 & $\begin{array}{l}24 \\
22 \\
21 \\
16.0 \\
14.8 \\
13.8\end{array}$ & $\begin{array}{l}18.9 \\
19.6 \\
16.9 \\
16.0 \\
15.1\end{array}$ & $\begin{array}{r}6.4 \\
5.8 \\
13.4 \\
7.7 \\
8.8 \\
6.9\end{array}$ & $\begin{array}{r}5.2 \\
5.1 \\
5.2 \\
5.4 \\
5.2 \\
\end{array}$ \\
\hline \multirow{2}{*}{ Month } & \multicolumn{4}{|c|}{ Million gallons a day } & \multirow{2}{*}{\multicolumn{2}{|c|}{$\begin{array}{c}\text { Second- } \\
\text { feet } \\
\text { (mean) }\end{array}$}} & \multicolumn{3}{|c|}{ Total ran-off } \\
\hline & Maximum & Min & imum & Mear & & & $\begin{array}{l}\text { Million } \\
\text { gallons }\end{array}$ & Acr & e-feet \\
\hline $\begin{array}{l}\text { November } 27-80 \\
\text { December } \\
\text { January } \\
\text { February } \\
\text { March } \\
\text { April } \\
\text { Mey }\end{array}$ & $\begin{array}{r}29 \\
330 \\
73 \\
\mathbf{9 1 3} \\
\mathbf{3 8 2} \\
\mathbf{2 4 6} \\
\mathbf{1 7 .} 0 \\
\mathbf{3 9}\end{array}$ & & $\begin{array}{r}22 \\
16.0 \\
7.9 \\
4.5 \\
13.8 \\
13.2 \\
5.8 \\
5.1\end{array}$ & $\begin{array}{l}24 . \\
57 . \\
22 . \\
64 . \\
63 . \\
54 . \\
9 . \\
10 .\end{array}$ & & $\begin{array}{l}37.9 \\
89.4 \\
35.3 \\
90.6 \\
98.9 \\
84.5 \\
14.7 \\
16.7\end{array}$ & $\begin{array}{r}98 \\
1,790 \\
700 \\
1,800 \\
1,980 \\
1,640 \\
29 \\
32\end{array}$ & & $\begin{array}{r}301 \\
5,500 \\
2,170 \\
5,580 \\
6,980 \\
5,080 \\
904 \\
804\end{array}$ \\
\hline siod & & & & & & & 8,6 & & 26,600 \\
\hline
\end{tabular}

$67754-32-7$ 


\section{HOROLII STREAM REAR HILO, HAWAII}

Location.-Water-stage recorder 500 feet above intake of Hilo Sugar Co.'s upper ditch, 2 miles from end of Kaiwiki road, and 10 miles from Hilo.

Drainage Area. - 8.3 square miles.

RmCords Availabli. - February, 1924, to June, 1929.

EXTREMEs.-Maximum discharge during year, 1,850 million gallons a day or 2,860 second-feet Feb. 15 (gage height, 12.47 feet); minimum, 1.0 million gallons a day or 1.6 second-feet June 29 .

1924-1929: Maximum discharge, 3,060 million gallons a day or 4,730 second-feet Nov. 21, 1924 (gage height, 16.5 feet, estimated from floodmarks); minimum, 0.1 million gallons a day or 0.2 second-foot Feb. 9 and Apr. 14, 1926.

REMARKs. - Records good for ordinary stages except those estimated, which are poor. High-stage records poor. No diversions above station.

Discharge, in million gallons a day, 1928-29

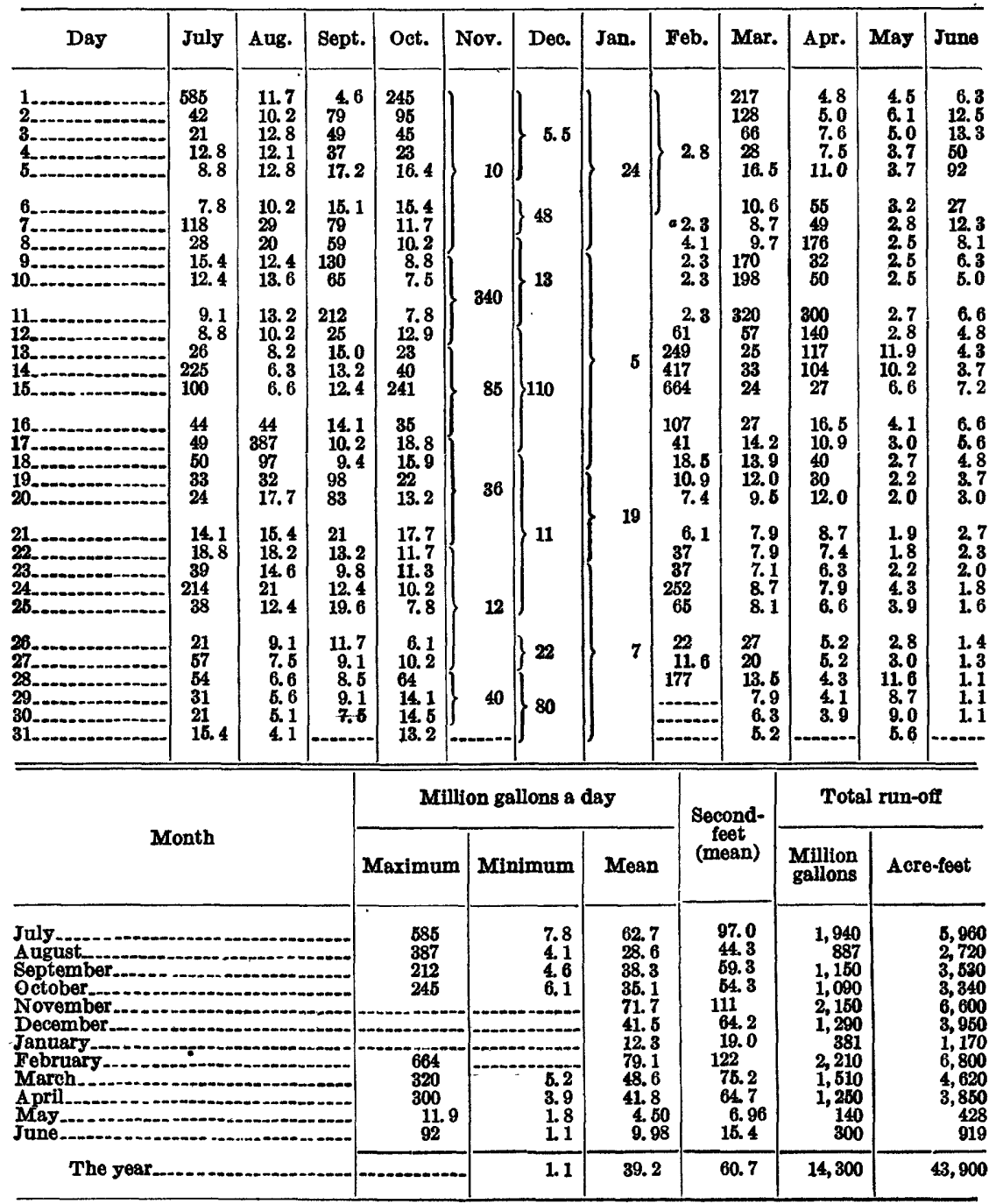

- Partly estimated. 
AWINI DITCH AT EAST HONOKANGIKI GULCH, NEAR FIUL, HAWAII

Locamion.-Water-stage recorder on Awini ditch at flume across East Honokaneiki Gulch, $4 \frac{1}{2}$ miles southeast of Niulii.

REcords avalLABLE.-October, 1927, to June, 1929.

EXTREMEs.-Maximum discharge during year, 32 million gallons a day or 50 second-feet Sept. 10 (gage height, 3.49 feet); minimum, 1.0 million gallons a day or 1.6 second-feet Mar. 12 .

1928-29: Maximum discharge, 32 million gallons a day or 50 secondfeet Dec. 28, 1927, and Sept. 10, 1928 (gage height, 3.51 feet); mi nimum, that of Mar. 12, 1929.

Remarks.-Records fair. Awini ditch diverts water from all streams between Waikaloa and Honokane. Regulated by head gates and spillways.

Discharge, in million gallons a day, 1928-29

\begin{tabular}{|c|c|c|c|c|c|c|c|c|c|c|c|c|}
\hline Day & July & Aug. & Sept. & Oct. & Nov. & Dec. & Jan. & Feb. & Mar. & Apr. & May & June \\
\hline $\begin{array}{l}1 .- \\
2 .- \\
3 .- \\
4- \\
5 .-\end{array}$ & $\begin{array}{l}26 \\
21 \\
24 \\
17.5 \\
12.8\end{array}$ & $\begin{array}{l}16.0 \\
17.6 \\
23 \\
25 \\
26\end{array}$ & $\begin{array}{r}8.5 \\
12.4 \\
19.9 \\
22.9 \\
18.4\end{array}$ & $\begin{array}{l}9.0 \\
7.6 \\
7.1 \\
7.1 \\
8.5\end{array}$ & $\begin{array}{r}4.5 \\
10.6 \\
21 \\
19.0 \\
16.8\end{array}$ & $\begin{array}{l}6.3 \\
6.2 \\
5.8 \\
5.3 \\
7.5\end{array}$ & $\begin{array}{l}16.0 \\
15.6 \\
30 \\
25 \\
23\end{array}$ & $\begin{array}{r}12.8 \\
11.0 \\
10.2 \\
9.6 \\
9.0\end{array}$ & $\begin{array}{l}7.6 \\
7.9 \\
9.6 \\
8.5 \\
7.6\end{array}$ & $\begin{array}{r}8.5 \\
13.2 \\
19.2 \\
21 \\
15.2\end{array}$ & $\begin{array}{r}9.0 \\
9.8 \\
11.4 \\
9.2 \\
8.5\end{array}$ & $\begin{array}{r}5.1 \\
5.2 \\
7.1 \\
12.4 \\
15.1\end{array}$ \\
\hline $\begin{array}{r}6 \\
7 \\
8 \\
9 \\
10 \\
10\end{array}$ & $\begin{array}{l}10.8 \\
24 \\
22 \\
18.9 \\
14.3\end{array}$ & $\begin{array}{l}19.8 \\
24 \\
24 \\
18.8 \\
21\end{array}$ & $\begin{array}{l}17.5 \\
17.5 \\
20 \\
23 \\
25\end{array}$ & $\begin{array}{r}9.1 \\
15.1 \\
11.8 \\
8.5 \\
7.1\end{array}$ & $\begin{array}{l}9.0 \\
18.6 \\
22 \\
30 \\
27\end{array}$ & $\begin{array}{l}28 \\
18.4 \\
15.2 \\
20 \\
20\end{array}$ & $\begin{array}{l}23 \\
22 \\
22 \\
22 \\
20\end{array}$ & $\begin{array}{l}13.5 \\
19.5 \\
20 \\
21 \\
23\end{array}$ & $\begin{array}{l}8.0 \\
7.6 \\
7.6 \\
8.8 \\
8.0\end{array}$ & $\begin{array}{l}10.0 \\
13.7 \\
17.5 \\
17.5 \\
24\end{array}$ & $\begin{array}{l}8.2 \\
16.1 \\
22 \\
21 \\
14.4\end{array}$ & $\begin{array}{r}14.9 \\
10.2 \\
7.4 \\
6.2 \\
7.9\end{array}$ \\
\hline $\begin{array}{l}11 \\
12 \\
13 \\
14 \\
15\end{array}$ & $\begin{array}{l}15.5 \\
14.2 \\
21 \\
19.0 \\
19.2\end{array}$ & $\begin{array}{l}21 \\
19.2 \\
17.2 \\
17.5 \\
11.4\end{array}$ & $\begin{array}{l}28 \\
23 \\
18.4 \\
15.1 \\
14.8\end{array}$ & $\begin{array}{r}6.2 \\
5.8 \\
6.5 \\
11.8 \\
18.1\end{array}$ & $\begin{array}{l}28 \\
18.3 \\
19.2 \\
28 \\
26\end{array}$ & $\begin{array}{l}19.8 \\
26 \\
24 \\
28 \\
26\end{array}$ & $\begin{array}{l}19.2 \\
16.3 \\
15.1 \\
15.1 \\
15.1\end{array}$ & $\begin{array}{l}20 \\
20 \\
19.2 \\
26 \\
19.2\end{array}$ & $\begin{array}{r}6.4 \\
3.9 \\
10.0 \\
25 \\
22\end{array}$ & $\begin{array}{l}28 \\
28 \\
25 \\
24 \\
20\end{array}$ & $\begin{array}{l}17.7 \\
19.2 \\
21 \\
22 \\
19.2\end{array}$ & $\begin{array}{r}17.9 \\
17.5 \\
12.8 \\
8.5 \\
9.2\end{array}$ \\
\hline $\begin{array}{l}16 \\
17 \\
18 \\
19 \\
20\end{array}$ & $\begin{array}{l}14.2 \\
15.4 \\
23 \\
24 \\
20\end{array}$ & $\begin{array}{l}13.6 \\
23 \\
26 \\
23 \\
17.5\end{array}$ & $\begin{array}{r}14.3 \\
11.4 \\
14.0 \\
\times 24 \\
619\end{array}$ & $\begin{array}{l}9.0 \\
6.6 \\
5.8 \\
5.8 \\
4.3\end{array}$ & $\begin{array}{l}25 \\
21 \\
20 \\
20 \\
19.2\end{array}$ & $\begin{array}{l}24 \\
17.5 \\
27 \\
25 \\
21\end{array}$ & $\begin{array}{l}15.1 \\
16.0 \\
15.1 \\
26 \\
19.2\end{array}$ & $\begin{array}{l}17.8 \\
21 \\
19.2 \\
14.0 \\
27\end{array}$ & $\begin{array}{l}20 \\
18.4 \\
17.5 \\
15.1 \\
13.5\end{array}$ & $\begin{array}{l}17.2 \\
22 \\
26 \\
20 \\
17.5\end{array}$ & $\begin{array}{r}18.4 \\
15.8 \\
11.6 \\
9.6 \\
9.0\end{array}$ & $\begin{array}{r}15.5 \\
15.1 \\
14.2 \\
9.6 \\
7.6\end{array}$ \\
\hline $\begin{array}{l}21 \\
22 \\
23 \\
24\end{array}$ & $\begin{array}{l}15.1 \\
20 \\
25 \\
28 \\
25\end{array}$ & $\begin{array}{l}15.1 \\
13.5 \\
14.9 \\
22 \\
17.1\end{array}$ & $\begin{array}{r}r 14 \\
a 10.0 \\
6.6 \\
6.2 \\
9.0\end{array}$ & $\begin{array}{l}6.4 \\
6.4 \\
5.0 \\
4.3 \\
6.0\end{array}$ & $\begin{array}{r}16.8 \\
11.4 \\
9.0 \\
9.8 \\
10.0\end{array}$ & $\begin{array}{l}26 \\
24 \\
20 \\
25 \\
30\end{array}$ & $\begin{array}{l}15.7 \\
30 \\
28 \\
23 \\
20\end{array}$ & $\begin{array}{r}22 \\
18.0 \\
14.6 \\
6.4 \\
7.6\end{array}$ & $\begin{array}{l}12.8 \\
12.1 \\
18.9 \\
13.5 \\
12.8\end{array}$ & $\begin{array}{l}19.2 \\
16.0 \\
14.2 \\
18.4 \\
16.0\end{array}$ & $\begin{array}{r}8.0 \\
7.6 \\
8.8 \\
12.8 \\
10.0\end{array}$ & $\begin{array}{l}6.2 \\
4.9 \\
3.6 \\
3.5 \\
8.5\end{array}$ \\
\hline $\begin{array}{l}26 \\
27 \\
28 \\
30\end{array}$ & $\begin{array}{l}25 \\
22 \\
20 \\
20 \\
18.4 \\
16.0\end{array}$ & $\begin{array}{r}16.4 \\
17.5 \\
16.3 \\
11.4 \\
11.4 \\
9.0\end{array}$ & $\begin{array}{r}8.0 \\
5.8 \\
4.8 \\
11.7 \\
12.8 \\
\end{array}$ & $\begin{array}{l}8.6 \\
7.0 \\
9.6 \\
7.6 \\
7.1 \\
\text { 5. } 3\end{array}$ & $\begin{array}{l}9.0 \\
8.5 \\
8.0 \\
7.6 \\
7.1\end{array}$ & $\begin{array}{l}27 \\
19.2 \\
19.2 \\
19.2 \\
16.8 \\
14.2\end{array}$ & $\begin{array}{l}18.6 \\
16.0 \\
15.9 \\
24 \\
16.0 \\
13.5\end{array}$ & $\begin{array}{r}6.6 \\
9.0 \\
8.6 \\
\\
\end{array}$ & $\begin{array}{r}10.8 \\
9.6 \\
9.0 \\
8.5 \\
8.0 \\
8.0\end{array}$ & $\begin{array}{l}12.8 \\
10.8 \\
10.8 \\
10.8 \\
10.2 \\
\end{array}$ & $\begin{aligned} 11.4 \\
8.8 \\
7.1 \\
6.6 \\
5.8 \\
5.3\end{aligned}$ & $\begin{array}{r}3.6 \\
3.6 \\
3.6 \\
3.1 \\
8.0 \\
-2\end{array}$ \\
\hline
\end{tabular}

\begin{tabular}{|c|c|c|c|c|c|c|}
\hline \multirow{2}{*}{ Month } & \multicolumn{3}{|c|}{ Million gallons a day } & \multirow{2}{*}{$\begin{array}{l}\text { Second- } \\
\text { feet } \\
\text { (mean) }\end{array}$} & \multicolumn{2}{|c|}{ Total run-off } \\
\hline & Maximum & Minimum & Mean & & $\begin{array}{l}\text { Million } \\
\text { gallons }\end{array}$ & Acre-feet \\
\hline $\begin{array}{l}\text { July } \\
\text { Angust } \\
\text { September } \\
\text { October } \\
\text { November. } \\
\text { December. } \\
\text { January } \\
\text { February } \\
\text { March } \\
\text { April } \\
\text { May } \\
\text { June }\end{array}$ & $\begin{array}{l}28 \\
26 \\
28 \\
18.1 \\
30 \\
30 \\
30 \\
27 \\
25 \\
28 \\
22 \\
17.9\end{array}$ & $\begin{array}{r}10.8 \\
9.0 \\
4.8 \\
4.3 \\
4.5 \\
5.3 \\
13.5 \\
6.4 \\
3.9 \\
8.5 \\
\mathbf{5 . 3} \\
\mathbf{3 . 1}\end{array}$ & $\begin{array}{l}19.7 \\
18.4 \\
15.2 \\
7.87 \\
16.7 \\
19.7 \\
19.7 \\
15.9 \\
11.5 \\
17.6 \\
12.4 \\
8.77\end{array}$ & $\begin{array}{l}30.5 \\
28.5 \\
23.5 \\
12.2 \\
25.8 \\
30.5 \\
30.5 \\
24.6 \\
17.8 \\
27.2 \\
19.2 \\
13.6\end{array}$ & $\begin{array}{l}611 \\
569 \\
455 \\
244 \\
500 \\
612 \\
612 \\
446 \\
357 \\
527 \\
385 \\
263\end{array}$ & $\begin{array}{r}1,870 \\
1,750 \\
1,400 \\
749 \\
1,540 \\
1,870 \\
1,870 \\
1,370 \\
1,090 \\
1,620 \\
1,180 \\
807\end{array}$ \\
\hline The year.................. & 30 & 3.1 & 15.3 & 23.7 & 5,580 & 17,100 \\
\hline
\end{tabular}




\section{BAST HONORANEIKI INTAKE TO AWINI DITCH AT EAST HONOKANEIKI GULCH, MEAR NIULII, HAWAII}

Location.-Water-stage recorder on intake tunnel delivering water from East Honokaneiki Gulch to Awini ditch on west side of gulch, $41 / 2$ miles southeast of Niulii.

Records Avallable.-October, 1927, to June, 1929.

EXTREMEs.-Maximum discharge during year, 10.1 million gallons a day or 15.6 second-feet Sept. 2 (gage height, 1.33 feet); minimum, 0.18 million gallons a day or 0.28 second-foot Oct. 19, 20, June 29 .

1928-29: Maximum discharge, that of Sept. 2, 1928; minimum, 0.11 million gallons a day or 0.17 second-foot Nov. 3, 4, 1927.

REMARKs.-Records good. Diverts water from East Honokaneiki Gulch to Awini ditch.

Discharge, in million gallons a day, 1928-29

\begin{tabular}{|c|c|c|c|c|c|c|c|c|c|c|c|c|}
\hline Day & July & Aug. & Sept. & Oct. I & Nov. & eec. & Jan. & Feb. & Mar. & Apr. & May & June \\
\hline .......... & \begin{tabular}{l|}
4.2 \\
5.2 \\
5.9 \\
4.2 \\
3.8
\end{tabular} & $\begin{array}{l}1.3 \\
2.1 \\
4.0 \\
4.8 \\
3.6\end{array}$ & $\begin{array}{l}1.6 \\
6.6 \\
5.4 \\
4.3 \\
2.8\end{array}$ & $\begin{array}{r}0.44 \\
.40 \\
.35 \\
.35 \\
.31\end{array}$ & \begin{tabular}{l|}
0.20 \\
1.9 \\
3.8 \\
3.1 \\
2.7
\end{tabular} & $\begin{array}{r}0.47 \\
.48 \\
.48 \\
.48 \\
2.1\end{array}$ & \begin{tabular}{l|}
1.2 \\
1.2 \\
5.2 \\
3.7 \\
2.5
\end{tabular} & $\begin{array}{r}1.8 \\
1.1 \\
.9 \\
.9 \\
.8\end{array}$ & $\begin{array}{l}1.2 \\
1.0 \\
.8 \\
.57 \\
.57\end{array}$ & $\begin{array}{l}0.53 \\
1.64 \\
1.3 \\
1.6 \\
1.3\end{array}$ & $\begin{array}{l}0.9 \\
.9 \\
.9 \\
.8 \\
.8\end{array}$ & $\begin{array}{l}1.0 \\
2.7 \\
2.2 \\
1.0\end{array}$ \\
\hline 6. & \begin{tabular}{l|}
3.6 \\
5.2 \\
3.6 \\
3.2 \\
1.6
\end{tabular} & $\begin{array}{l}2.0 \\
3.4 \\
3.2 \\
1.8 \\
2.7\end{array}$ & $\begin{array}{l}3.5 \\
6.1 \\
4.6 \\
4.7 \\
\text { b.3 }\end{array}$ & $\begin{array}{l}.8 \\
4.2 \\
1.0 \\
.48 \\
.35\end{array}$ & $\begin{array}{l}1.4 \\
4.1 \\
4.7 \\
6.9 \\
6.5\end{array}$ & $\begin{array}{l}4.9 \\
3.4 \\
3.5 \\
5.5 \\
5.6\end{array}$ & $\begin{array}{l}5.5 \\
2.7 \\
4.7 \\
3.6 \\
2.1\end{array}$ & $\begin{array}{l}.8 \\
4.0 \\
5.8 \\
2.7 \\
2.0\end{array}$ & $\begin{array}{l}.57 \\
.57 \\
.66 \\
.8 \\
.26\end{array}$ & $\begin{array}{l}.7 \\
1.3 \\
4.6 \\
2.8 \\
4.3\end{array}$ & 2.4 & $\begin{array}{l}.5 \\
.4 \\
.3 \\
.3 \\
.3\end{array}$ \\
\hline$\ldots$ & $\begin{array}{l}1.4 \\
1.6 \\
4.0 \\
3.7 \\
3.2\end{array}$ & \begin{tabular}{r|}
3.7 \\
3.2 \\
2.4 \\
2.5 \\
.9
\end{tabular} & $\begin{array}{l}6.0 \\
4.4 \\
3.1 \\
2.8 \\
1.6\end{array}$ & $\begin{array}{l}.31 \\
.31 \\
.56 \\
1.3 \\
.7\end{array}$ & $\begin{array}{l}4.4 \\
3.8 \\
2.5 \\
2.4 \\
2.4\end{array}$ & $\begin{array}{l}5.2 \\
5.8 \\
5.2 \\
5.9 \\
5.0\end{array}$ & $\begin{array}{l}1.6 \\
1.4 \\
2.1 \\
6.0 \\
4.5\end{array}$ & $\begin{array}{l}1.3 \\
1.2 \\
3.0 \\
3.4 \\
4.3\end{array}$ & $\begin{array}{l}.7 \\
2.4 \\
3.3 \\
2.7 \\
2.4\end{array}$ & $\begin{array}{l}4.6 \\
4.2\end{array}$ & $\begin{array}{l}3.6 \\
2.9 \\
3.6 \\
4.3 \\
3.3\end{array}$ & $\begin{array}{l}2.6 \\
3.9 \\
1.2 \\
.66 \\
2.9\end{array}$ \\
\hline 口n- & $\begin{array}{l}1.6 \\
1.4 \\
4.0 \\
4.3 \\
1.9\end{array}$ & $\begin{array}{l}2.1 \\
6.4 \\
3.9 \\
2.2 \\
1.5\end{array}$ & $\begin{array}{l}1.3 \\
1.2 \\
1.4 \\
5.9 \\
2.3\end{array}$ & $\begin{array}{l}.40 \\
.31 \\
.35 \\
.20 \\
.20\end{array}$ & $\begin{array}{l}2.4 \\
2.2 \\
3.5 \\
4.3 \\
3.1\end{array}$ & $\begin{array}{l}3.8 \\
2.4 \\
4.9 \\
3.7 \\
2.4\end{array}$ & $\begin{array}{l}2.8 \\
1.6 \\
1.4 \\
6.2 \\
2.5\end{array}$ & $\begin{array}{l}4.5 \\
5.3 \\
4.6 \\
2.7 \\
6.0\end{array}$ & $\begin{array}{l}1.8 \\
1.8 \\
1.7 \\
1.5 \\
1.3\end{array}$ & $\begin{array}{l}2.4 \\
2.4 \\
4.5 \\
3.4 \\
2.2\end{array}$ & 1.2 & $\begin{array}{r}2.9 \\
1.6 \\
1.3 \\
.9 \\
.8\end{array}$ \\
\hline 5. & $\begin{array}{l}2.6 \\
5.1 \\
6.2 \\
4.3 \\
2.1\end{array}$ & $\begin{array}{l}1.2 \\
1.0 \\
1.8 \\
2.8 \\
2.7\end{array}$ & $\begin{array}{r}1.3 \\
1.2 \\
1.1 \\
.9 \\
.9\end{array}$ & $\begin{array}{l}.20 \\
.22 \\
.22 \\
.26 \\
.26\end{array}$ & $\begin{array}{l}2.4 \\
1.4 \\
1.1 \\
.9 \\
.8\end{array}$ & $\begin{array}{l}3.6 \\
3.9 \\
2.9 \\
2.9 \\
2.9\end{array}$ & $\begin{array}{l}1.7 \\
6.3 \\
5.4 \\
3.2 \\
2.6\end{array}$ & $\begin{array}{l}5.4 \\
5.7 \\
3.4 \\
.35 \\
.40\end{array}$ & $\begin{array}{l}1.2 \\
1.1 \\
1.4 \\
1.2 \\
1.1\end{array}$ & $\begin{array}{l}1.9 \\
1.5 \\
1.3 \\
1.7 \\
1.4\end{array}$ & $\begin{array}{l}1.2 \\
1.1 \\
1.1 \\
.57 \\
.54\end{array}$ & .7 \\
\hline (1) & $\begin{array}{l}5.4 \\
3.2 \\
2.3 \\
2.6 \\
1.8 \\
1.4\end{array}$ & $\begin{array}{l}2.6 \\
2.6 \\
1.4 \\
1.8 \\
1.7\end{array}$ & $\begin{array}{l}.8 \\
.66 \\
.62 \\
.57 \\
.53\end{array}$ & $\begin{array}{l}.26 \\
.22 \\
.22 \\
.22 \\
.20\end{array}$ & $\begin{array}{l}.8 \\
.62 \\
.57 \\
.48 \\
.44\end{array}$ & $\begin{array}{l}2.8 \\
2.4 \\
2.4 \\
2.1 \\
1.3 \\
1.0\end{array}$ & $\begin{array}{l}2.4 \\
2.4 \\
1.6 \\
3.5 \\
2.8 \\
1.9 \\
-\end{array}$ & \begin{tabular}{c|}
.8 \\
1.0 \\
.8 \\
\hdashline .8. \\
..-
\end{tabular} & $\begin{array}{l}.9 \\
.9 \\
.8 \\
.53 \\
.47 \\
.48\end{array}$ & $\begin{array}{l}1.2 \\
1.0 \\
1.0 \\
.9 \\
.9\end{array}$ & $\begin{array}{l}.48 \\
.44 \\
.40 \\
.35 \\
.31 \\
.26\end{array}$ & $\begin{array}{l}.4 \\
.4 \\
.2 \\
.1\end{array}$ \\
\hline \multirow{2}{*}{\multicolumn{4}{|c|}{ Mont }} & \multicolumn{4}{|c|}{ Million gallons a day } & \multirow{2}{*}{\multicolumn{2}{|c|}{$\begin{array}{c}\text { Second- } \\
\text { feet } \\
\text { (mean) }\end{array}$}} & \multicolumn{3}{|c|}{ Total run-of } \\
\hline & & & & Iaximum & \multicolumn{2}{|c|}{ Minimum } & Mean & & & $\begin{array}{l}\text { Million } \\
\text { gallons }\end{array}$ & \multicolumn{2}{|c|}{ Acre-feet } \\
\hline \multicolumn{4}{|c|}{$\begin{array}{l}\text { July } \\
\text { August } \\
\text { September } \\
\text { October- } \\
\text { November... } \\
\text { December } \\
\text { January } \\
\text { February } \\
\text { March } \\
\text { April } \\
\text { May } \\
\text { June }\end{array}$} & $\begin{array}{l}6.2 \\
6.4 \\
6.6 \\
4.2 \\
6.9 \\
6.9 \\
6.3 \\
6.0 \\
3.3 \\
4.6 \\
4.3 \\
3.9\end{array}$ & \multicolumn{2}{|c|}{$\begin{array}{r}1.4 \\
.9 \\
: 53 \\
.20 \\
.20 \\
.47 \\
1.2 \\
. \quad .35 \\
.26 \\
.53 \\
.26 \\
.18\end{array}$} & $\begin{array}{l}3.37 \\
2.59 \\
2.78 \\
.510 \\
.2 .49 \\
3.21 \\
3.11 \\
2.68 \\
1.18 \\
2.20 \\
1.63 \\
1.10\end{array}$ & \multicolumn{2}{|c|}{$\begin{array}{l}\text { 5. } 21 \\
4.01 \\
4.30 \\
.789 \\
3.85 \\
4.97 \\
4.81 \\
4.15 \\
1.83 \\
3.40 \\
2.62 \\
1.70 \\
\end{array}$} & $\begin{array}{c}105 \\
80.2 \\
83.5 \\
15.8 \\
74.8 \\
99.4 \\
96.3 \\
75.0 \\
76.7 \\
66.0 \\
60.0 \\
33.1\end{array}$ & \multicolumn{2}{|r|}{$\begin{array}{l}321 \\
246 \\
256 \\
49 \\
229 \\
305 \\
296 \\
230 \\
112 \\
203 \\
155 \\
101\end{array}$} \\
\hline The year. & & & & 6.9 & & .18 & 2.24 & & 47 & 816 & & 500 \\
\hline
\end{tabular}




\section{KOHALA DITCH AT POLOLU, NRAR NIULI, HAWAII}

Location.-Water-stage recorder on open section of ditch in Pololu Valley, just below boundary between Bishop Estate land of Honokane and territorial land of Pololu, 2\%/4 miles above mouth of Pololu Stream, and 4 miles south of Niulii.

Reco RDS AVAILABLE.-August, 1927, to June, 1929.

ExTremes.- Maximum discharge during year, 55 million gallons a day or 85 second-feet Dec. 6 (gage height, 3.66 feet); minimum, 1.2 million gallons a day or 1.9 second-feet Dec. 9 .

1928-29: Maximum discharge, that of Dec. 6, 1928; minimum, that of Dec. 9, 1928.

Remarks.- Records good. Regulated by head gates. Kohala ditch receives flow of Awini ditch and diverts from all streams west of Honokane.

Discharge, in million gallons a day, 1928-29

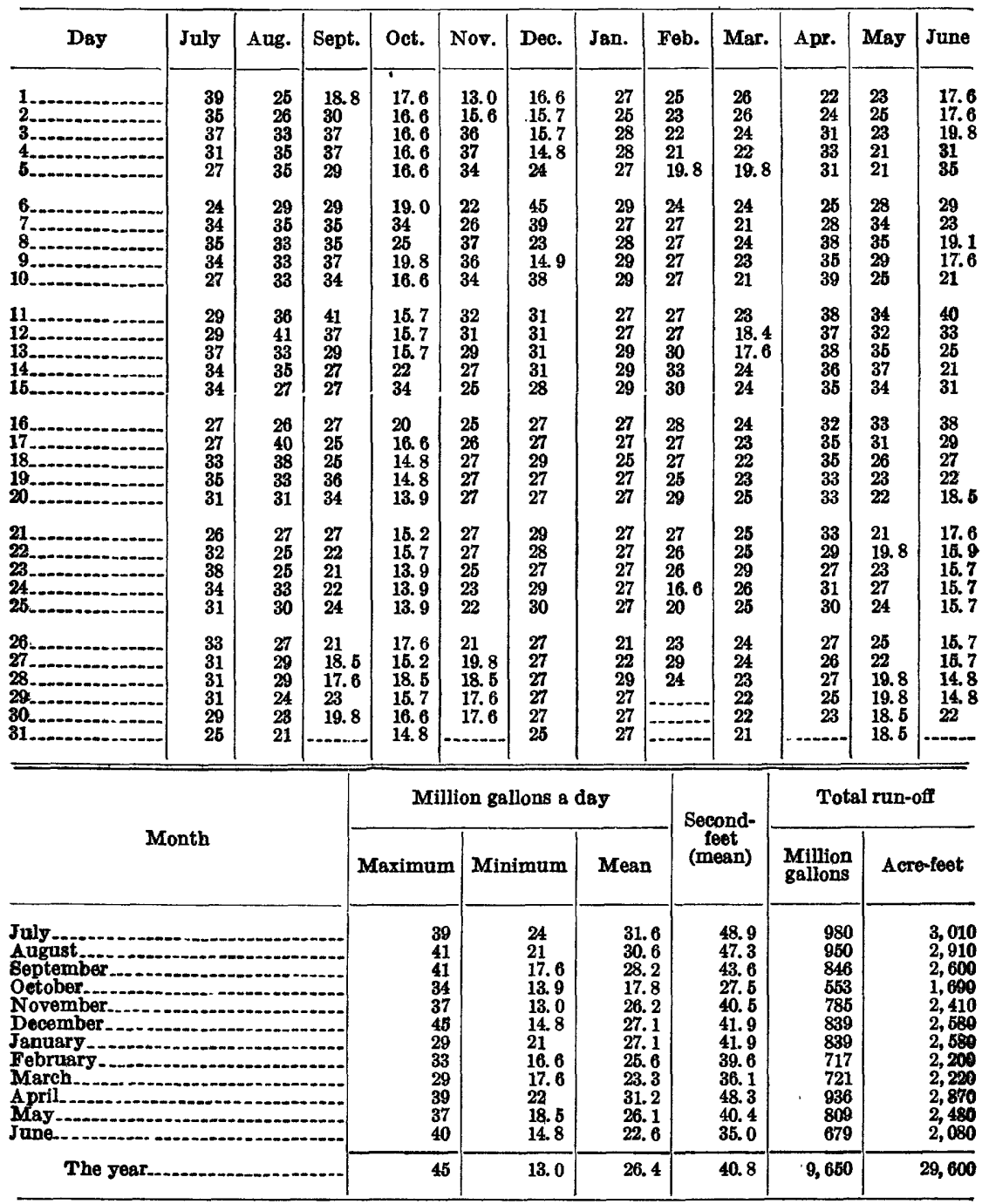




\section{KEHENA DITCH NEAR KOHALA, HAWAII}

Location.-Water-stage recorder at old Honokane weir, just below head of West Branch of Honokanenui Gulch and 81/2 miles southeast of Kohala.

Records avaILABLe. - December, 1917, to November, 1919; April, 1928, to June, 1929.

Extremes.-Maximum discharge during period, 67 million gallons a day or 104 second-feet Jan. 22 (gage height, 1.42 feet); minimum, 0.5 million gallons a day or 0.8 second-foot Apr. 28-30, Dec. 4, 5 .

1917-1919, 1928-29: Maximum discharge, 86 million gallons a day or 133 second-feet Jan. 27, 1918 (gage height, 2.16 feet); ditch dry June 7, 1919.

Remarks.-Records poor, owing to poor work of observer. . Regulated by several gates above station. Intake on Honokanenui Stream 2 miles above station.

Discharge, in million gallons a day, 1928-29

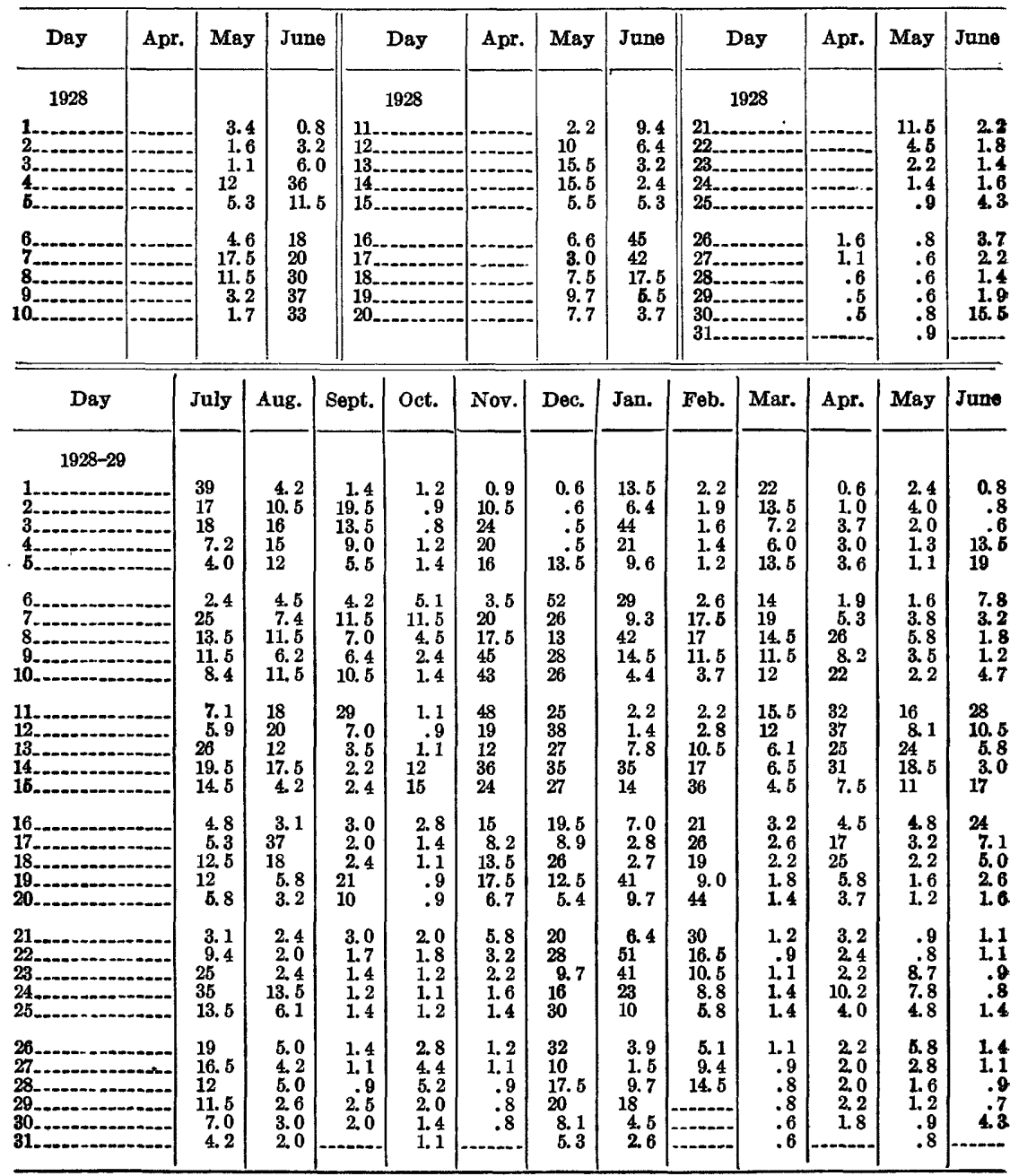


Monthly discharge of Kehena ditch near Kohala, Hawaii, 1928-29

\begin{tabular}{|c|c|c|c|c|c|c|}
\hline \multirow{2}{*}{ Month } & \multicolumn{3}{|c|}{ Million gallons a day } & \multirow{2}{*}{$\begin{array}{l}\text { Second- } \\
\text { feet } \\
\text { (mean) }\end{array}$} & \multicolumn{2}{|c|}{ Total run-off } \\
\hline & Maximum & Minimum & Mean. & & gallons & Acre-feet \\
\hline $\begin{array}{l}1928 \\
\text { April (5 days) } \\
\text { Juy- }\end{array}$ & $\begin{array}{r}1.6 \\
47.5\end{array}$ & $\begin{array}{r}0.8 \\
.6 \\
.8\end{array}$ & $\begin{array}{c}0.86 \\
5.48 \\
12.4\end{array}$ & $\begin{array}{r}1.33 \\
8.48 \\
19.2\end{array}$ & $\begin{array}{c}{ }^{4.3} .3 \\
170 \\
372\end{array}$ & $\begin{array}{r}13 \\
521 \\
1,140\end{array}$ \\
\hline $\begin{array}{l}\text { July } \\
\text { August } \\
\text { Geptember. } \\
\text { October } \\
\text { November } \\
\text { December } \\
\text { January } \\
\text { February } \\
\text { March } \\
\text { Aprl } \\
\text { May-29 }\end{array}$ & $\begin{array}{l}39 \\
37 \\
29 \\
15 \\
48 \\
52 \\
51 \\
44 \\
22 \\
37 \\
24 \\
28\end{array}$ & $\begin{array}{r}2.4 \\
2.0 \\
.9 \\
.8 \\
.8 \\
.5 \\
1.4 \\
1.2 \\
.6 \\
.6 \\
.8 \\
.6\end{array}$ & $\begin{array}{c}13.4 \\
9.22 \\
6.25 \\
2.96 \\
14.0 \\
18.8 \\
15.8 \\
12.5 \\
6.45 \\
9.87 \\
4.98 \\
5.72\end{array}$ & $\begin{array}{c}20.7 \\
14.3 \\
9.67 \\
4.58 \\
21.7 \\
29.1 \\
24.4 \\
19.3 \\
9.98 \\
15.3 \\
7.71 \\
8.85\end{array}$ & $\begin{array}{c}416 \\
286 \\
188 \\
91.8 \\
419 \\
582 \\
489 \\
349 \\
200 \\
296 \\
154 \\
172\end{array}$ & $\begin{array}{r}1,270 \\
877 \\
576 \\
282 \\
1,290 \\
1,790 \\
1,500 \\
1,070 \\
614 \\
909 \\
474 \\
627\end{array}$ \\
\hline The jear. & 62 & .5 & 9.98 & 15.4 & 3,640 & 11,200 \\
\hline
\end{tabular}

MTSCELIANRO OS MEASUREMERT

A measurement of Awini ditch, tributary to Kohala ditch, at Awini weir, at Honokanenui Gulch, near Niulii, on Mar. 12, 1929, showed a discharge of 8.74 second-feet or 5.65 million gallons a day. 


\section{INDEX}

$A$

Accuracy of data and computed results.

Acre-foot, definition of.

Alo Stream near Fuelo, Maui.

Anahola ditch above Kaneha Reservoir, near Kealia, Kauai.

Anahola River near Kealia Kaugi

Awini ditch at East Honokanelki Gulch, near Niulii, Hawail

discharge measurement of

East Honokaneiki intake to, at East Honokaneiki Gulch, near Niulii, Hawaii.

$\mathrm{C}$

Center ditch below Kolea Reservoir, near Huelo, Maui............................

Computations, results of, accuracy of.........

Control, definition of

Cooperation, record of ..............................

\section{D}

Data, accuracy of explanation of

East Manoe ditch near Eonoluln, Oahu...... East Walluaiki Stream near Keanse, Maui.. East Wailuanui Stream near Keanae, Maui. Eleele, Kauai, Hanapepe ditch near.

Hanapepe River near

\section{F}

First Division ditch, $\mathrm{Oahu}$, discharge measurement of

H

Haiku ditch at Manawai Gulch, near Peahi, Maui.

Haipuaena Stream near Huelo, Mani

Halawa Stream near Halawa, Molokai......

Hanalei, Kauai, Hanalei River near.

Lumahai River near

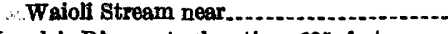

Hanalei River at elevation 625 feet, near Hanalel, Kauai.................................

Henapepe ditch at Koula, near Eleele, Kauai. discharge measurements of.
Page

6-7

4

76

24

3

0

93
97

94
Hanapepe diversion ditches, Kauai, discharge measurements of.......... 28-29

Hanapepe River at Koula, near Eleele, Kauai.

discharge measurements of................. 29

Hanawi Stream near Nahiku, Maui......... 58

Hawaii, gaging-station records on ............. 90-97

Hawaiian Homes Commission tunnel, Molokai, discharge measurement of... $\quad 51$

Hiilaniwai Stream, Oahu, discharge measurement of.............................. 40

Hilo, Hawaii, Honolii Stream near......... 92

Kapehn Stream near..................... 91

Wailuku River near....................... 90

Hiloa ditch, Kauai, discharge measurement of. 29

Honokahau Stream near Honokahan, Maui. $\quad 52$

Honokawai ditch near Lahaina, Maui........ 53

Honolii Stream near Hilo, Hawaii............- 92

Honolulu, Oahu, East Branch of Manoa Stream near........................ 36

East Manoa ditch near...................... 37

Kalihi stream near....................... 33

Moanalua Stream near.................... 32

Nuuanu Stream near....................... 34

Pukele Stream near........................ 38

Waiomao Stream near....................... 39

West Branch of Manoa Stream near...... 35 Honolulu ditch, Maui, discharge measurements of

Honomanu Stream near Keanae, Mani:..... 68

Honopou Stream near Huelo, Maui.......... 85

Hoolawaliilii Stream near Huelo, Maui.....- 88

Hoolawanui Stream near Huelo, Maui....... 84

Huelo, Maui, Alo Stream near............... 76

Center ditch near............................ 80

Haipuaens Stream near................... 69

Honopou Stream near................... 85

Hoolawaliilii Stream near.................. 83

Hoolawanui Stream near................... 84

Kailus Stream near........................ 82

Kauhikoa ditch near. .................... 88

Koolan ditch near...................... 75

Manuel Luis ditch near..................... 73

Nailillihaele Stream near................... 81

New Homakua ditch near................. 87

Puohoksmos intake of Koolau ditch near. $\quad 72$

Puohokamos Stream near................. 71

Spreckels ditch near...................... 70, 74, 79

Waikamoi Stream near..................... 77

Wailos ditch near........................ $\quad 86$

\section{I}

Investigations, authority for. 
Page

Kagiea Stream near Kailua, Maui.

Kahalawe Stream, Right Branch of, near Kipahulu, Maui

Kallua, Maui, Kaaiea Stream near...........

Kailua Stream near Huelo, Maui.

Kalaupapa, Molokal, Waikolu Stream near..

Kalihi Stream near Honolulu, Oahu.

Kanaha ditch near Lihue, Kauai ................

Kanaha Stream above pipe-line intake near Lahaina, Maui.

Kapahi ditch near Kealia, Kauai.

Kapaula Stream near Nahiku, Maui.........

Kapehu Stream at Pithonua, near Eilo, Hawaii

Kauai, gaging-station records on

Kauhikoa ditch at Opana weir, near Huelo, Maul.

Kaukonahua Stream, Left Branch of North Fork of, near Wahiawa, Oahu...-

Right Branch of North Fork of, near Wahiawa, Oahu

Kawaikol Stream near Waimea, Kauai.......

Kealia, Kauai, Anahola ditch near.

Anahola River near.

Kapahi ditch near.

Keanae, Maui, East Wailuaiki Stream near.

East Walluanui Stream near..............

Honomanu Stream near.

Koolau ditch near

West Kopilliula Stream near

West Koplliula Stream near...............-

Wést Wailuanui Stream near

Kehena ditch near Kohala, Hawaii........... 96-97

Kekahe ditch at camp No. 1, near Waimea, Kauai.

below tunnel No. 12, near Waimea, Kauai ....................................

Eipahulu, Maui, Oheo Stream near..........

Right Branch of Kahalawe Stream near...

Koaie Stream at elevation 3,700 feet, near Waimea, Kauai...........................

Kohala, Hawail, Kehena ditch near ....... 96-97

Kohala ditch at Pololu, near Niulii, Hawaii.-

Kokee ditch near Waimea, Kauai.............

Koolau ditch at Nahiku weir, near Nahiku, Maui.

at Wahinepe, near Huelo, Maui.

near Keana, Maui.........................

Puohokamoa intake of, near Huelo, Maui.

$\mathbf{L}$

Lahaina, Maui, Honokawai ditch near......

Kanahe Stream near..................................

Lanipuni Stream near Pelekunu, Molokai

LihuB, Rausi, East Branch of North Fork of Wailua River near

Kanaha ditch near

North Fork of Wailua River near

South Fork of Wailua River near.........

Luluku Stream, Oahu, discharge measurement of.

14
Lumahai River, Kauai, discharge measurements of.

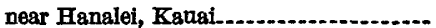

M

Manoa Stream, East Branch of, near Honolulu, Oahu

West Branch of, near Honolulu, Oahu... Manuahi, Kauai, discharge meesurement of.Manuel Luis ditch at Puohokamoa Gulch, near Huelo, Maui.

Maui, gaging-station records on............ 52-80

Million gallons, definition of ................. 4

Moanalua Stream near Honolulu, Oahu..... 32

Molokai, gaging-station records on............ 41-51

\section{$\mathbf{N}$}

Nahiku, Mari, Hanawi Stream near........ Kapaula Stream near........................

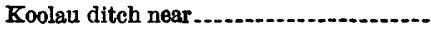

Waiohue Stream near.....................

Nallilihaele Steam near Huelo, Maui........

New Hamakua ditch at Honopou, near Huelo, Maui.

Niulii, Hawaii, A wini ditch near..............

East Honokaneiki intake to Awini ditch near.

Kohala ditch near

North Halawa Stream, Oahu, discharge measurement of.

North Luluku ditch, Oahu, discharge measurement of .

diversion ditch of, discharge meesurement of.

Nuuanu Stream below Reservoir No. 2 waste way, near Honolulu, Oahu......

0

Oahu, gaging-station records on

Oheo Stream at elevation 1,500 feet, near Kipahulu, Maui

Olowalu ditch near Olowalu, Mavi

$\mathbf{P}$

Papalaua Stream near Wailau, Molokai..... 42-43

Peahi, Maui, Haiku ditch near................ 89

Pelekunu, Molokai, Lanipuni Stream near. - 48-49

Pelekunu Stream near Pelekunu, Molokai-. $\quad 47$

Puohokamoa Stream near Euelo, Maui..... 71

Publications on stream flow, list of........... 7

Pukele Stream near Honolulu, Oahu........ 38

Pulena Stream near Wailau, Molokal........ 46

$\mathbf{8}$

Second-foot, definition of ......................... Spreckels ditch at Haipusena weir, near Huelo, Maui

58

59

60

81

87

93

94 95

at Wahinepe, near Huelo, Maui.

below Kaaiea Gulch, near Huelo, Maui. Stage-discharge relation, definition of..........

\section{6}

4




\section{Page}

Terms, definition of ................................

W

Wahiawa, Oahu, Left Branch of North Fork of Kaukonshua Stream near.

Right Branch of North Fork of Kankonahua Stream near

Walahulu Stream above Koaie Stream, near Waimea, Kauai

Waiakeakua Stream near Wailau, MolokaL. 44-45

Waialae River at elevation 3,700 feet, near Waimea, Kanai

Waihanau Stream, Molokai, discharge measurement of

Waikamol Stream above Wailoa ditch, near Huelo, Maui.

Waikolu Stream at pipe-line crossing near Kalaupepa, Molokai

Wailan, Molokai, Papalana Stream near...... 42-43

Pulens Stream near -........................ 46

Waiakeakua Stream near................... 44-45

Waileia Stream, Molokai, discharge measuroment of.

Wailoa ditch at Honopou, near Huelo, Maui.
Wallua River, East Branch of North Fork of, near Lthue, Kauai.

North Fork of, at elevation 650 feet, near Lihue, Kauai.

South Fork of noar Lihue, Kaugi -a--

Walluku River above Hilo Boarding Bchool ditch intake, near Hilo, Hawail..

Waimea, Kaual, Kawaikoi Stream near..... 9

Kelksha ditch near........................... 14-15

Koaie Stream near........................ 12

Kokee ditch near.......................... 10

Waiahulu Stream near................... 11

Waialae River near........................ 13

Waimee River near.................... 8

Waimes River below Kekaha ditch intake, near Waimea, Kauai .............. . 8

Waiohue Stream near Nahiku, Maui........ 61

Waioll Stream near Hanalei, Kaual........... 26

Waiomao Stream above Pukele Stream, near Honolulu, Oahu................... 39

West Kopiliula Stream near Keanae, Maui.- 62

West Wailuaiki Stream near Keanae, Maui. $\quad 64$

West Wailuanui Stream near Keanae, Maui. $\quad 66$

Work, division of............................. 7

scope of 


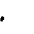


. 